\title{
Cluster jets for quasi-phase matching in high-order harmonic generation
}




\section{CLUSTER JETS FOR QUASI-PHASE MATCHING IN HIGH-ORDER HARMONIC GENERATION}

CLUSTER JETS VOOR QUASI-PHASE MATCHING

IN HOGE ORDE HARMONISCHE GENERATIE

Yin TAO 
Ph.D. graduation committee:

Chairman \& secretary:

Prof. dr. ir. J.W.M. Hilgenkamp

University of Twente

Promotor:

Prof. dr. K.-J. Boller

University of Twente

Co-promotor:

Dr. ing. H.M.J. Bastiaens

University of Twente

\title{
Members:
}

Prof. dr. M. Kovacev

Prof. dr. H.P. Urbach

Prof. dr. C. Fallnich

Prof. dr. J.L. Herek

Prof. dr. P.W.H. Pinkse

\author{
Gottfried Wilhelm Leibniz Universität Hannover \\ Delft University of Technology \\ Westfälische Wilhelms-Universität Münster \\ \& University of Twente \\ University of Twente \\ University of Twente
}

The work described in this thesis was carried out at the Laser Physics and Nonlinear Optics group, Department of Science and Technology, MESA+ Institute for Nanotechnology, University of Twente, P.O. Box 217, 7500 AE Enschede, The Netherlands. This research was supported by the Dutch Technology Foundation STW, which is part of the Netherlands Organization for Scientific Research (NWO), and partly funded by the Ministry of Economic Affairs (Project No. 10759).

Copyright (C) 2016 by Yin Tao, Enschede, The Netherlands.

ISBN: 978-90-365-4273-9

DOI: $10.3990 / 1.9789036542739$

https://doi.org/10.3990/1.9789036542739

Printed by Gildeprint, Enschede, The Netherlands. 


\title{
Cluster JeTS FOR QUASI-PHASE MATCHING IN HIGH-ORDER HARMONIC GENERATION
}

\section{Dissertation}

\author{
to obtain
}

the doctor's degree at the University of Twente, on the authority of the rector magnificus, Prof. dr. T.T.M. Palstra,

on account of the decision of the graduation committee, to be publicly defended on Friday, December $16^{\text {th }}, 2016$ at 14:45

by

\section{Yin TAO}

born on February $28^{\text {th }}, 1986$

in Shanghai, The People's Republic of China 
This dissertation is approved by:

Promotor: Prof. dr. K.-J. Boller

Co-promotor: Dr. ing. H.M.J. Bastiaens 
To my family. 



\section{Contents}

Summary ix

Samenvatting $\quad$ xiii

1 Introduction 1

1.1 Outline of the thesis. . . . . . . . . . . . . . . . . 10

2 Theoretical background of cluster formation and high-order harmonic generation 11

2.1 Cluster formation . . . . . . . . . . . . . . . 12

2.1.1 Basic concepts of gas flow . . . . . . . . . . . . . . . 12

2.1.2 Quasi one-dimensional model of the gas flow . . . . . . . . . . . . 16

2.1.3 Theoretical approach to cluster formation . . . . . . . . . . . . . . 19

2.2 High-order Harmonic generation . . . . . . . . . . . . . . . . . . 21

2.2.1 Single-atom response . . . . . . . . . . . . . . . . 21

2.2.2 Macroscopic response . . . . . . . . . . . . . . 27

3 Experimental preparation $\quad 39$

3.1 Cluster jet characterization setup . . . . . . . . . . . . . . . 40

3.1.1 Supersonic nozzle . . . . . . . . . . . . . . . . . . . 40

3.1.2 Interferometry and Rayleigh scattering setup . . . . . . . . . . . . 42

3.2 High-order harmonic generation setup . . . . . . . . . . . . . . . 43

3.2.1 High power femtosecond laser system . . . . . . . . . . . . . . . . . . . . 44

3.2.2 HHG target chamber. . . . . . . . . . . . . . . . . . 46

3.2.3 Harmonic diagnostic system. . . . . . . . . . . . . . . . . . 47

4 Revisiting argon cluster formation in a planar gas jet for high-intensity laser matter interaction $\quad \mathbf{5 1}$

4.1 Introduction . . . . . . . . . . . . . . . . . 52

4.2 Experiments . . . . . . . . . . . . . . . . 55

4.2 .1 Interferometry. . . . . . . . . . . . . . . 56

4.2.2 Rayleigh scattering. . . . . . . . . . . . . . . . . 57

4.3 Theoretical model. . . . . . . . . . . . . . . . . . . . . 60

4.3.1 Nozzle geometry and reservoir conditions . . . . . . . . . . . 60

4.3.2 Conservation equations . . . . . . . . . . . . . . . . . 60

4.3.3 Surface tension model . . . . . . . . . . . . . . . . . . 63

4.3.4 Liquid mass density model. . . . . . . . . . . . . . . . . . . . . . 64

4.3.5 Growth rate model . . . . . . . . . . . . . . . . . . . . . . 64

4.3.6 Model variation sensitivity . . . . . . . . . . . . . . . . . 66

4.3.7 Baseline model and results. . . . . . . . . . . . . . . . 68 
4.4 Average cluster size . . . . . . . . . . . . . . . . . . 70

4.5 Conclusion . . . . . . . . . . . . . . . . . 71

5 Cluster size dependence of high-order harmonic generation 73

5.1 Introduction . . . . . . . . . . . . . . . . . 74

5.2 Experimental setup . . . . . . . . . . . . . . . . . 76

5.3 Results and Discussion . . . . . . . . . . . . . . . . . . . . . . . 77

5.4 Conclusion . . . . . . . . . . . . . . . . . . . . 85

6 A temporal quasi-phase matching model for high-order harmonic genera$\begin{array}{ll}\text { tion } & 87\end{array}$

6.1 Introduction . . . . . . . . . . . . . . . . . . . . . . . . . 88

6.2 One-dimensional time-dependent model for HHG . . . . . . . . . . . . . 89

6.3 Time-dependence of phase matching in a homogeneous medium . . . . . 92

6.4 HHG in a periodic density modulated medium . . . . . . . . . . . . . . 96

6.5 Results and Discussion of HHG with QPM ～. . . . . . . . . . . . . . . . 99

6.6 Conclusion . . . . . . . . . . . . . . . . . . . 104

$\begin{array}{lll}7 \text { Conclusion and outlook } & 105\end{array}$

Appendix

A Nozzle design $\quad 111$

$\begin{array}{lll}\text { B HHG setup } & 113\end{array}$

$\begin{array}{lll}\text { C Solution method for conservation equations } & 117\end{array}$

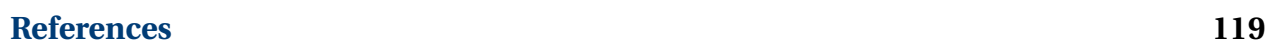

$\begin{array}{ll}\text { List of Publications } & 131\end{array}$

$\begin{array}{ll}\text { Acknowledgements } & 135\end{array}$ 


\section{SUMMARY}

High-order harmonic generation (HHG) is an extremely nonlinear optical process that generates extreme ultraviolet (XUV) radiation or even soft X-rays, with a high, unprecedented spatial and temporal coherence. These unique, remarkable properties have enabled a wealth of applications including coherent diffraction imaging, detection of electron processes in various systems and frequency comb spectroscopy. However, HHG is intrinsically inefficient, and the highest-ever achieved efficiency is rather modest $(\sim$ $10^{-5}$ ) and was only achieved in a limited spectral region of the high-order harmonic $(\mathrm{HH})$ spectrum where the phase-matching condition could be fulfilled. The highest harmonic order (shortest wavelength) that can be efficiently generated is limited through the loss of the phase-matching condition due to a large ionization fraction induced by the required high drive laser intensities. A potential solution to efficiently generate shorterwavelength radiation is using quasi-phase matching (QPM). QPM is a technique that introduces spatial modulation of the generation process along the entire medium, enabling HHG in the in-phase regions and suppressing HHG in the out-of-phase regions.

We propose and investigate, in this thesis, a novel scheme for achieving QPM in HHG via a density modulated cluster jet, as opposed to a modulated gas jet. Such a cluster jet can be provided from a supersonic expansion of a noble gas through a slit nozzle, where clusters are aggregated via van-der-Waals forces. Compared to a gas jet, a density modulation is expected to be more easily achieved by placing an array of wires (grid) on the top of the nozzle. The main reason for this expectation is that clusters as compared to gas atoms (monomers) exhibit a much reduced transverse diffusion due to their relatively large mass, which is promising to create a rather high density contrast behind the grid. Thereby also a finer-scale modulation behind an array of wires appears available, which can be important for increasing the efficiency via QPM. Moreover, a higher nonlinear response in clusters as compared to atomic gaseous media was claimed by previous studies, which might provide an increased $\mathrm{HH}$ output.

However, in our initial experiments, we did not observe any enhanced HH output using previously fabricated grids with various different modulation periods. These experiments suggest that, even if using a density modulated cluster jet remains promising for achieving QPM in HHG, an according demonstration first requires a thorough investigation of several basic and essential aspects.

These aspects are, first, the liquid mass fraction, $g$, which describes the ratio of the number of atoms aggregated in clusters as compared to the total number of atoms in the jet. In most of the previous experiments that investigated cluster jets for HHG, this fraction was assumed to be equal to unity without further justification. However, the actual value of the liquid mass fraction, $g$, according to our work, lies below $20 \%$. Therefore, there is generally an overestimation of the average cluster size and the number density of clusters in the jet. Secondly, as a consequence of using incorrect values for $g$, the interpretation of the strength of the nonlinear optical response of clusters from previ- 
ous HHG experimental data appears quite questionable. Finally, the variation of phase mismatch on an ultrashort time scale, due to the rapidly increasing ionization fraction during the drive laser pulse has not been taken into account in previous calculations and experiments for selecting the proper modulation period for QPM. A particular, selected modulation period may phase match HHG only for an extremely short time interval. However, this does not necessarily mean that the $\mathrm{HH}$ output pulse energy is maximized as well. These aspects need to be investigated before an experimental demonstration of QPM becomes possible for an efficient generation of shorter-wavelength radiation via HHG.

In this thesis, we investigated these three aspects. First, we studied the process of cluster formation in a supersonic slit nozzle, and it aimed on determining the average size, $\langle N\rangle$, of clusters, as well as the liquid mass fraction, $g$, in the jet. We presented a comprehensive modelling of cluster formation and systematically investigated all influences of various critical physical assumptions for the gas condensation in a supersonic nozzle using argon as an example. Using the proposed baseline model, we showed that the liquid mass fraction is very insensitive with regards to the named variations in this model, which justifies the usage of this model to derive the averaged cluster size from experimental data. The average cluster size, $\langle N\rangle$, was retrieved from interferometric and Rayleigh scattering measurements by using the calculated liquid mass fraction, $g$, from the baseline model. The essential experimental parameters for cluster growth in a supersonic jet, for instance, the stagnation pressure (pressure of the gas reservoir), are usually summarized in the so-called Hagena parameter, $\Gamma^{*}$. We determined that the average cluster size, $\langle N\rangle$, follows a modified power law for higher values of the Hagena parameter in the range of $1.8 \times 10^{4}$ to $2.5 \times 10^{5}$, which is the range that is of highest importance for HHG. Our power law complements the previously found scaling law for the Hagena parameter, extending the total range of predictable average cluster sizes from $\Gamma^{*}=10^{3}$ to $2.5 \times 10^{5}$. This translates into a range of the average cluster size, $\langle N\rangle$, that can be predicted with high reliability, extending from about a thousand to almost ten million atoms per cluster.

Secondly, we performed a detailed experimental study on HHG in a supersonic argon jet. In order to identify and separate the contributions from clusters versus that of gas monomers in the jet to the generation of high-order harmonics, we characterized the harmonic spectra over a broad range of stagnation pressures (between 300 mbar to 35 bar) at two different reservoir temperatures (303 K and $363 \mathrm{~K}$ ). Varying the temperature allowed us to maintain the same average cluster size, $\langle N\rangle$, at different total atomic number densities in the jet. We proposed a simple model to interpret the dependence of the $\mathrm{HH}$ yield on the total atomic number density in the jet. Using the calculated value of $g$, which is below $20 \%$ for our experimental conditions, we derived the relative nonlinear $\mathrm{HH}$ response of clusters with different average sizes. We observed that, below an average cluster size, $\langle N\rangle$, of about 1000 atoms, HHG in clusters shows the same efficiency as in gas monomers. Only with larger clusters $(\langle N\rangle>1000$ atoms per cluster), HHG becomes less efficient. We also found no changes of the cut-off energy in the measured HH spectra, which indicates that the three-step model for HHG in atoms remains valid for HHG in clusters.

Lastly, we investigated quasi-phase matching for HHG, specifically aiming at deter- 
mining a proper quasi-phase matching modulation period for achieving the optimum HH output pulse energy. We developed a one-dimensional, dynamic QPM model for HHG in a gaseous medium (argon) with a spatially periodic density modulation. From the model, we analyzed the wave-vector mismatch and harmonic dipole amplitude during the entire drive pulse, and found that it is not possible to achieve QPM for any selected generated high-order harmonics during the entire drive laser pulse using a fixed periodic structure. This is due to the ultrafast temporal dependence of the coherence length (wave-vector mismatch) at the elevated, strongly ionizing intensities required for generating short output wavelengths. We showed that simply choosing the modulation period according to the coherence length calculated at the peak intensity of the laser pulse when the highest harmonic dipole amplitude is reached, is not the optimum choice for achieving the highest $\mathrm{HH}$ output pulse energy. According to our model, the optimum HH output pulse energy is obtained when transient QPM is provided in the leading edge of the drive laser pulse.

The basic understanding of the cluster formation, the nonlinear $\mathrm{HH}$ response of clusters and the temporal dependence of the phase mismatch in the generating media is essential, and the progress achieved in our work on these aspects forms a solid and important foundation towards exploitation of QPM in HHG. Yet, further developments are required to determine whether quasi-phase matched HHG at shorter wavelengths is feasible by introducing a periodic density modulation in a mixture of clusters and gas monomers. 



\section{SAMENVATting}

Hoge orde harmonische generatie (HHG) is een extreem niet-lineair optisch proces dat extreem ultraviolet (EUV) licht genereert met een enorm hoge ruimtelijke en temporele coherentie. Dankzij deze unieke en opmerkelijke eigenschappen heeft HHG diverse toepassingen, waaronder beeldvorming door middel van coherente diffractie, detectie van elektronenprocessen in verscheidene systemen en frequentiekamspectroscopie. Echter, HHG is inherent een inefficiënt proces en de hoogste gerapporteerde efficiëntie is gering $\left(\sim 10^{-5}\right)$. Deze efficiëntie werd alleen behaald voor een beperkt golflengtegebied van het hoge harmonische spectrum $(\mathrm{HH})$ waarvoor aan de phasematchingeis voldaan kon worden. De hoogste harmonische orde (kortste golflengte) die efficiënt gegenereerd kan worden is beperkt omdat, door de hoge ionisatiegraad van het medium, veroorzaakt door de benodigde hoge drive laser intensiteiten, niet aan de phasematchingeis kan worden voldaan. Een mogelijke oplossing om efficiënt korte golflengtes te genereren is het gebruik van quasi-phase matching (QPM). Deze techniek houdt in dat er een ruimtelijke modulatie van het HHG-proces wordt aangebracht over de lengte van het medium, waardoor HHG plaatsvindt in de 'in-fase' gebieden en onderdrukt wordt in de 'uit-fase' gebieden.

In dit proefschrift introduceren en onderzoeken wij een nieuw concept om QPM in HHG te realiseren, waarbij we in plaats van een gemoduleerde gas jet een dichtheid gemoduleerde cluster jet gebruiken. De clusters worden gevormd in een supersonische expansie van een edelgas via een spleetvormige nozzle, waarin de gasatomen aggregeren tot clusters. In vergelijking met een gas jet, zal het naar verwachting eenvoudiger zijn om een dichtheidsmodulatie aan te brengen in de cluster jet door een rooster van draden bovenop de nozzle te plaatsen. De belangrijkste reden hiervoor is dat clusters, in vergelijking met gasatomen (monomeren), vanwege hun relatief grote massa een veel lagere transversale diffusie vertonen waardoor een hoog dichtheidscontrast achter het rooster haalbaar lijkt. Ook lijkt het mogelijk om op deze manier achter het rooster de clusterdichtheid op een fijnere schaal te moduleren, wat van belang kan zijn voor het verhogen van de efficiëntie door middel van QPM. Daarnaast is volgens voorgaand onderzoek de niet-lineaire respons van clusters hoger in vergelijking met atomaire gasvormige media, wat kan resulteren in een toename van de HH-energieopbrengst.

Echter, in onze eerste HHG-experimenten zagen we geen verhoogde energieopbrengst van clusterjets met verschillende modulatieperiodes. Dit wijst erop dat, zelfs als QPM van HHG in gemoduleerde clusterjets veelbelovend is voor QPM van HHG, er voor een demonstratie eerst een grondige studie nodig is naar diverse fundamentele en essentiële aspecten.

Deze aspecten zijn ten eerste de vloeistofmassafractie (liquid mass fraction), $g$, die de verhouding aangeeft tussen het aantal in clusters aggregeerde atomen en het totale aantal atomen in de supersonische jet. In de meeste eerder uitgevoerde experimenten aan HHG in clusterjets is aangenomen dat deze fractie gelijk is aan 1, zonder enige ver- 
dere rechtvaardiging. Echter, de werkelijke waarde van de vloeistofmassafractie ligt volgens ons onderzoek onder de $20 \%$. Dit betekent dat in het algemeen de gemiddelde clustergrootte en de deeltjesdichtheid van clusters in de jet wordt overschat. Ten tweede, door het gebruik van onjuiste waarden voor $g$, wordt ook de interpretatie van de sterkte van de niet-lineaire respons van clusters uit data van voorgaande HHG-experimenten zeer discutabel. Tenslotte is bij het vaststellen van de juiste modulatieperiode voor QPM in voorgaande berekeningen en experimenten geen rekening gehouden met de variatie van de phase mismatch op ultrakorte tijdschaal. Voor een specifiek gekozen modulatieperiode is phase matching van HHG alleen mogelijk gedurende een extreem kort tijdsinterval. Echter, dit betekent niet dat daarmee ook de HH-energieopbrengst maximaal is. Daarom moeten deze aspecten onderzocht worden voordat een experimentele demonstratie van QPM voor een efficiënte generatie van hogere harmonischen mogelijk is.

In dit proefschrift hebben wij deze drie aspecten nader onderzocht. Allereerst hebben wij de vorming van clusters in een supersonische, spleetvormige nozzle bestudeerd, met als doel het bepalen van de gemiddelde clustergrootte, $\langle N\rangle$, en de vloeistofmassafractie, $g$,in de jet. We presenteren een uitgebreide modellering van de vorming van clusters waarin een systematische studie is gedaan naar de invloed van een aantal cruciale fysische aannames over de gascondensatie in een supersonische nozzle, met argon als voorbeeld. Met het door ons voorgestelde baselinemodel hebben wij aangetoond dat de vloeistofmassafractie erg ongevoelig is voor variaties in de diverse fysische aannames, wat het gebruik van het model voor het bepalen van de gemiddelde clustergrootte uit de experimentele data rechtvaardigt. De gemiddelde clustergrootte, $\langle N\rangle$, is verkregen uit interferometrische metingen en Rayleigh scattering metingen, waarbij gebruik gemaakt is van een vloeistofmassafractie, g, zoals berekend met het baselinemodel. De essentiële experimentele parameters voor clustergroei in een supersonische jet, zoals bijvoorbeeld de stagnatiedruk (druk in het gasreservoir), zijn doorgaans beschreven met de zogenaamde Hagena parameter, $\Gamma^{*}$. Wij hebben vastgesteld dat de gemiddelde clustergrootte, $\langle N\rangle$, een aangepaste machtsfunctie volgt voor hogere waarden van de Hagenaparameter in het bereik van $1.8 \times 10^{4}$ tot $2.5 \times 10^{5}$, wat het belangrijkste bereik is voor HHG. Deze machtsfunctie die wij hebben bepaald complementeert de eerder gevonden spellingswet voor de Hagena parameter, waarmee het totale bereik waarover gemiddelde clustergrootte voorspeld kan worden zich nu uitstrekt van $\Gamma^{*}=10^{3}$ tot $2.5 \times 10^{5}$. Dit vertaalt zich naar een gemiddelde clustergrootte, $<\mathrm{N}>$, die met hoge betrouwbaarheid over een bereik van ongeveer duizend tot bijna tien miljoen atomen per cluster voorspeld kan worden.

Als tweede hebben wij een gedetailleerde experimentele studie uitgevoerd naar HHG in een supersonische argon jet. Om de afzonderlijke bijdragen aan de generatie van $\mathrm{HH}$ van clusters en gasmonomeren in de jet te identificeren en van elkaar te scheiden hebben we de harmonische spectra gekarakteriseerd voor een grote reeks stagnatiedrukken (tussen 300 mbar en 35 bar) bij twee verschillende reservoirtemperaturen ( $303 \mathrm{~K}$ en 363 $\mathrm{K})$. Door de reservoirtemperatuur te variëren is het mogelijk om bij verschillende totale atomaire deeltjesdichtheden in de jet toch clusters met dezelfde gemiddelde clustergrootte, $\langle N\rangle$, te genereren. We hebben een eenvoudig model opgesteld waarmee de afhankelijkheid van de $\mathrm{HH}$-opbrengst van de totale atomaire deeltjesdichtheid in de jet 
wordt beschreven. Met behulp van de berekende waarde voor $g$, die voor onze experimentele omstandigheden onder de $20 \%$ ligt, hebben wij de relatieve niet-lineaire $\mathrm{HH}$ respons van clusters met verschillende groottes vastgesteld. We komen tot de conclusie dat onder een gemiddelde clustergrootte, $\langle N\rangle$, van ongeveer 1000 atomen, de generatie van hogere harmonischen in clusters met dezelfde efficiëntie verloopt als in gasmonomeren. Alleen voor grotere clusters, met meer dan 1000 atomen per cluster, verloopt de HHG minder efficiënt. Daarnaast zagen we in de gemeten $\mathrm{HH}$-spectra geen verschil in cutoffenergie, waaruit blijkt dat het driestappen model voor HHG in atomen ook van toepassing is voor HHG in clusters.

Tenslotte hebben we een onderzoek uitgevoerd naar quasi-phase matching van HHG met het specifieke doel om de modulatieperiode waarmee een zo hoog mogelijke $\mathrm{HH}-$ energieopbrengst kan worden gerealiseerd te bepalen. We hebben een eendimensionaal, dynamisch QPM-model ontwikkeld voor HHG in een gasvormig medium (argon) waarin een ruimtelijke periodieke modulatie van de dichtheid is aangebracht. Met behulp van dit model hebben wij de golfvectormismatch en harmonische dipoolamplitude gedurende de gehele drivepuls geanalyseerd. Hieruit blijkt dat bij gebruik van een vaste, periodieke structuur het voor geen enkele hoge orde harmonische mogelijk is om QPM te bewerkstelligen gedurende de gehele drivelaserpuls. Dit is het gevolg van de ultrasnelle tijdsafhankelijkheid van de coherentielengte (golfvectormismatch) die voorkomt uit het gebruik van de hoge, sterk ioniserende intensiteiten die nodig zijn voor het genereren van korte golflengtes. We hebben aangetoond dat het eenvoudigweg kiezen van een modulatieperiode op basis van de coherentielengte berekend voor de piekintensiteit van de drivelaserpuls, waar de harmonische dipoolamplitude het hoogst is, niet de optimale strategie is voor het behalen van de hoogste energieopbrengst. Volgens ons model wordt de optimale $\mathrm{HH}$-energieopbrengst bereikt als er gedurende korte tijd QPM wordt aangebracht in de voorflank van de drivelaserpuls.

Een fundamenteel begrip van clustervorming, van de niet-lineaire $\mathrm{HH}$-respons van clusters en de tijdsafhankelijkheid van de phase mismatch in genererende media blijkt essentieel. De grote vorderingen die wij met ons onderzoek op deze onderwerpen hebben geboekt vormen een solide en belangrijke basis voor het gebruik van QPM bij HHG in de toekomst. Echter, om te bepalen of quasi-phase matching van HHG voor kortere golflengtes haalbaar is door een periodieke modulatie aan te brengen in de dichtheid van een mengsel van clusters en gasmonomeren zijn aanvullende ontwikkelingen noodzakelijk. 



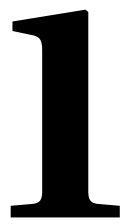

INTRODUCTION 
Shortly after Schawlow and Townes [1] had introduced the concept of the laser, promising the generation of light with extremely high, unprecedented spatial and temporal quality, the first laser was demonstrated in 1960 by Maiman [2]. One year later, second harmonic generation (SHG) was observed in a quartz crystal [3]. This opened up the remarkable field of nonlinear optics, where the quality of laser radiation can be transferred to other wavelengths and distributed across a dramatically increased spectral range. In the example of SHG, due to the sufficiently high intensities produced by the drive laser, a nonlinear polarization is induced in the crystal, which exhibits a quadratic dependence on the field strength of the laser beam. The light generated by this polarization is of identically high quality as the inducing laser radiation, however, at a much shorter wavelength. Since the observation of SHG during the last half century, an extensive range of nonlinear light-matter processes has been discovered and explored, thanks to the rapid evolution of laser technology and the development of new concepts. These are in particular Q-switching, mode-locking and Chirped Pulse Amplification (CPA), all aiming at increasing the drive laser intensity through pulsed generation with a short duration. Nowadays, nonlinear optical processes allow to generate light covering the entire spectral range from the infrared [4] to the X-ray region [5]. In particular, up-converting the coherent laser radiation to multiples of its original frequency, called harmonic generation, is often applied to generate radiation at short wavelengths, e.g., third harmonic generation in gaseous media [6] or fourth and fifth harmonic generation in crystals [7]. Because harmonic generation is a so-called parametric process, the output is again of the same high quality as the drive laser radiation. Harmonic generation is most efficiently achieved in crystals, but the shortest wavelengths that can be efficiently generated in solid materials typically reach down only to the ultraviolet region, to somewhat below $200 \mathrm{~nm}$. Generating high-quality radiation beyond the ultraviolet region, including the extreme ultraviolet (XUV, $10-100 \mathrm{~nm})$ or even in the soft X-ray $(0.1-10 \mathrm{~nm})$ spectral region has remained a significant challenge. There are a number of different alternatives for harmonic generation in the sense that short wavelengths can be obtained, for instance, using capillary discharge pumped X-ray lasers, laser-produced plasma sources, synchrotrons and free-electron lasers. However, for most applications in various fundamental and applied research fields, light sources are not only required to produce an output at a certain range of extremely short wavelengths in a maximally compact format, but have to meet high requirements concerning the quality of the radiation. To one part, this means that a maximum spatial coherence has to be provided which allows the generation of a diffraction limited output beam. The other, more important quality to be provided is full phase coherence across a maximally wide spectral range of tens of electron volts as to control the electric field down to an attosecond time scale. Pushing the limits of these qualities has enabled fundamentally new possibilities, such as precision measurements of the ionizaton potential of ${ }^{4} \mathrm{He}$ [8], or probing attosecond resolved electron dynamics [9] and molecular dynamics [10].

To compare various short-wavelength sources in these respects, let us look at X-ray lasers based on highly ionized plasmas. For instance, when exciting $\mathrm{Ne}$-like $\mathrm{Ar}^{8+}$ via a capillary discharge to generate laser radiation at $47 \mathrm{~nm}$ [11], the output has a relative low spatial and phase coherence, also lacking phase-to-phase reproducibility, due to its origin from amplified spontaneous emission. Similarly, laser-produced plasma 
sources (LPP) typically employ nanosecond pulsed laser beams with an intensity of up to $10^{18} \mathrm{~W} / \mathrm{cm}^{2}$ focused onto solid or liquid targets to obtain short-wavelength radiation. However, such sources are often fully incoherent, comprising spontanous emission emitted into all dimentions ( $4 \pi$ solid angle), which makes it difficult to efficiently collect or focus this radiation [12]. Regarding the spectro-temporal coherence, the excited ions decay and emit radiation via different spontaneous mechanisms including bremsstrahlung [13], radiative recombination [14] or fluorescence from particular transitions [15]. The generated spectrum from LPP sources usually contains a broad spectral background due to the thermal emission as well as several characteristic lines corresponding to certain specific energy transitions. In spite of many disadvantages associated with a low spatial and temporal coherence, due to lacking alternatives with similarly high average power, such sources are still of high importance, e.g., at around $13.5 \mathrm{~nm}$, as sources for next generation lithography machines [16].

A much better control of the radiation generation process is found in Synchrotron sources. Here, electromagnetic radiation is emitted when accelerated, high-energy, relativistic electrons are forced to change their direction of flight, e.g, in bending magnets or magnetic wigglers [17]. Since the electrons travel in the form of short bunches, synchrotrons generate short optical pulses, which are suitable for applications such as timeresolved imaging and spectroscopy [18]. Yet, the temporal resolution of those applications is usually limited by the pulse duration, which is typically as long as one nanosecond. Free-electron lasers (FEL) on the other hand, are typically based on higher electron peak currents, also using a beam of relativistic electrons. Under the influence of the magnetic field of the undulator and their own radiation, the electrons bunch together on a sub-wavelength scale which leads to a strongly increase of spatial and spectral coherence and also to the generation of short optical pulses providing extremely high intensities. FELs cover a wide spectral range from the microwave to the hard X-ray region [19]. The peak intensity of FELs is currently ten orders of magnitude higher than that of synchrotrons. Furthermore, the pulse duration is much shorter, down to a few hundred femtoseconds, which provides broad opportunities for exploring new fields in science including coherent diffractive imaging [20], extreme nonlinear optics [21] and multiphoton excitation in clusters [22]. However, the output from both synchrotrons and FELs is still based on, or develops from, random emission which is typically caused by shot noise in the electron beam. Therefore the output beam has a low shot-to-shot temporal coherence. Additionally, both sources are extremely large-scale facilities which are extremely costly to build and operate, often affordable only in international collaboration.

Currently, the most promising way to reach the XUV and soft X-ray regions with a high spatial and temporal coherence is High-order Harmonic Generation (HHG), an extreme nonlinear, parametric optical process discovered in 1987 [23, 24]. For generating high-order harmonics, typically femtosecond pulsed laser beams with a rather high peak intensity of $10^{13}-10^{14} \mathrm{~W} / \mathrm{cm}^{2}$ are focused into a gasous target [25]. The underlying physics of HHG can be intuitively understood using a simple three-step model [26, 27]: (i) Considering a single atom, first, a bound electron leaves the atomic potential via tunnel ionization, after the potential is distorted by the strong electric field of the laser. (ii) In the first half of the corresponding optical cycle, the tunnel ionized electron is accelerated away by the strong laser field and, upon field reversal in the other half of the optical 
cycle, is driven back to its parent ion. (iii) Finally, the electron recombines with its parent ion, converting the kinetic and ionization energy into an ultrashort burst of radiation with high photon energies. High-order harmonics exhibit a plateau-like behaviour such that relatively weak, but equally intense radiation is generated across a broad spectral range, followed by a sharp cut-off at some maximum light frequency.

Of central importance for the high quality radiation from HHG is that the generation is a parametric process, which reproduces the oscillating field of the drive laser at short wavelengths. Driven by femtosecond laser pulses, high-order harmonics exhibit an excellent temporal resolution, which has opened up many applications in material science, e.g., femtosecond or attosecond resolved detection of dynamical electronic processes in atoms [9], molecules [10] and solids [28]. Moreover, HHG sources generate fully coherent beams not only in the temporal domain but also in the spatial domain, which allows for experiments such as coherent diffractive imaging with high spatial resolution [29], investigating magnetic circular dichroism effects in metals [30], inspecting optical elements for EUV lithography [31] and seeding free-electron lasers to control their optical phase [32]. An additional advantage, specifically over free-electron lasers - the only sources that offer a coherent ultrashort output as well- is that HHG experiments can be performed in a standard optical laboratory in a table-top format.

In spite of these very beneficial properties of HHG in terms of the quality of the radiaiton, HHG is intrinsically a rather inefficient process. The conversion efficiency is typically lower than $10^{-7}$. The highest recorded value is approximately $10^{-5}$ [33], noting that this value was measured at a relatively low harmonic order, far below the cut-off energy for the atoms that were used. There are two main reasons for these low conversion efficiencies. One reason is the intrinsically low recombination rate of ionized electrons with their parent ions. The large majority of the electrons that tunnel away through the potential barrier simply miss their parent ions, instead of recombining with them and emitting high-order harmonic $(\mathrm{HH})$ radiation. This low recombination rate is depending on the intrinsic material properties of the generating medium, such as the spatial size of the ground state wave function in atoms, and cannot be easily improved. Another reason is that HHG with significant output intensity always involves an entire volume of, e.g., a gaseous medium comprising a huge number of individual atomic emitters. Therefore the $\mathrm{HH}$ output is strongly influenced by the mutual coherence of the atomic emitters and the superposition of their radiative contributions. Quantitatively, the latter is expressed as the so-called phase-matching condition. Fulfilling this condition, i.e., that ideally the phase of the $\mathrm{HH}$ radiation generated from all emitters inside the entire generating medium is brought to constructive interference, maximizes the overall output. Unfortunately, this situation is generally not easy to achieve. Efforts to avoid an undesired phase mismatch between the emitters, which is often characterized by the so-called wave-vector mismatch, $\Delta k$, include choosing a proper drive laser intensity, focusing geometry (e.g., waveguide geometry [34] or loose-focusing geometry [35]) and carefully adjusting the gas pressure. Optimizing these conditions allows relatively efficient HHG in the spectral regions of relatively long wavelengths with regard to the cutoff, where the drive laser ionizes the medium only to a small degree.

For generating shorter wavelengths with photon energies well beyond cut-off, there is, however, a fundamental limitation to the maximum harmonic order that can be gen- 
erated. According to the three-step model mentioned above, the cut-off in the HH spectrum (i.e., the highest generated photon energy that can be generated) is given by $E_{\text {cutoff }}=$ $I_{p}+3.17 U_{p}$. In this expression, $I_{p}$ is the ionization potential of the generating medium and $U_{p}$ is the ponderomotive energy. The ponderomotive energy, $U_{p} \propto I \lambda^{2}$, increases in proportion to the drive laser intensity, $I$, and quadratically with the driving laser wavelength, $\lambda$. According to this dependence, it seems straightforward that a shorter cut-off wavelength can be generated by using drive laser pulses with a longer wavelength, e.g., a mid-infrared laser instead of a Ti:Sa laser. Indeed, photons with energies up to one keV have been generated via HHG using a mid-infrared drive laser [36]. This was accompanied, however, with a dramatical decrease in conversion efficiency, equivalent to the generation only about $10^{6}$ photons per second. Both theoretical and other experimental studies confirm that the conversion efficiency scales unfavourably with the wavelength of the drive laser as $\lambda^{-6}[37,38]$.

Alternatively, simply increasing the drive laser intensity in the hope of achieving a shorter cut-off wavelength is problematic in a different manner. Since the HH output mainly originates from neutral atoms, the conversion efficiency drops when a large fraction of the medium becomes ionized. One can reduce the ionization fraction via using a medium with a higher ionization potential (e.g., Ne or He). However, compared to generating high-order harmonics in a medium with a relatively low ionization potential (e.g., Xe, Kr or Ar), the price paid then is a rather small single-atom dipole response, which again results in an overall lower conversion efficiency. The second main problem associated with increasing the drive laser intensity for an increased cut-off energy is that, due to the large number of generated free electrons, the phase-matching condition cannot be fulfilled any more. This results in destructive interference of the emitters and strongly decreases the conversion efficiency. Generally, as we shall explain in Chapter 2, there exists a critical ionization fraction for the generating medium (on the order of a few percent) that is not to be superseded as a prerequisite for efficient HHG. This critical ionization fraction limits the applied drive laser intensity and thereby the highest harmonic order (shortest wavelength) that can be efficiently generated in the $\mathrm{HH}$ spectrum.

When inspecting the reason for low output at high intensities causing a super-critical ionization, the individual atoms are still emitting radiation at shorter wavelengths. However, the superposition of their fields contributions does not build up but only oscillates spatially between a low and zero intensity along the propagation direction. The regions where the $\mathrm{HH}$ intensity decreases from a small value to zero are called out-of-phase regions. In order to improve the conversion efficiency for those higher order harmonics (shorter wavelengths), an alternative approach is often suggested which is so-called quasi-phase matching (QPM). This technique is widely applied in low-order nonlinear conversion, e.g., in SHG. In these cases, special crystals are used where it is possible to periodically and permanently reorient the crystal structure during fabrication.

However, such traditional QPM schemes cannot be transferred to HHG, since the media suitable for HHG are usually gaseous, which are generally isotropic and show an inversion symmetric response of the individual emitters. Specifically, it is difficult to reorient gaseous media into a desired direction. Nevertheless, QPM schemes based on similar ideas have been proposed, which maintain the HHG process in the in-phase regions and suspend (suppress) the generation in the out-of-phase regions. These schemes 
are based on introducing a periodic modulation of either the drive laser intensity or the density of the generating medium along the propagation direction.

Periodic modulation of the drive laser intensity to induce QPM has been experimentally demonstrated by using corrugated hollow waveguides [39], utilizing mode beating between multiple propagation modes in hollow waveguides [40] and applying a counterpropagating pulse train with pulses of appropriate widths and separation [41, 42]. However, these schemes are either limited by the lifetime of the hollow waveguide, or require critical alignment of the drive laser beams. In contrast, a spatially periodic modulation of the density of the generating medium to induce QPM appears relatively easy and has also been investigated, for instance, by propagating the drive laser pulses through several sequentially mounted gas nozzles that eject different types of gases [43-45]. Yet, these schemes demand a complex design of nozzle arrays, and a high accuracy is required in fabrication. The main drawback associated with these multi gas-jet arrays is that usually an insufficiently high density contrast is created, which lowers the efficiency that could be achieved with QPM. This is caused by the diffusion of the gas atoms into all directions due to their rather small masses, such that a mixing of the different species occurs. Accordingly, the smallest modulation periods that are typically preferable for efficiently generating the shortest-wavelength output via QPM are difficult to realize.

We propose and investigate, in this thesis, a promising alternative scheme for achieving QPM in HHG, namely, via a density modulated cluster jet, as opposed to a modulated gas jet. A density modulated cluster jet can be produced by simply placing an array of obtacles, e.g., thin wires, on the top of a supersonic slit nozzle [46]. Within the nozzle, upon supersonic expansion, gas atoms or molecules aggregate via van-der-Waals forces to form clusters.

The motivation of using clusters instead of gas is the following. Firstly, clusters possess a relatively large mass and size, other than gas atoms (monomers). This reduces transverse diffusion and offers promise for density modulation behind obstacles with a sufficiently high contrast. Thereby also a finer-scale modulation behind an array of obstacles would be achievable, which should be of benefit for increasing the efficiency via quasi-phase matching.

The second advantage of clusters versus gas monomers may be a higher output per atom. This claim has been laid based on previous experiments that concluded a higher nonlinear response in clusters, even growing with the cluster size, as compared to atomic gaseous media $[47,48]$.

A density modulated cluster jet was first proposed for generating an axially modulated plasma waveguide as a means of direct acceleration of electrons by a high-intensity laser pulse (laser wakefield acceleration) [49]. In the latter context, a sharp plasma density contrast behind an array of wires has indeed been recently observed [50], when the average cluster size $\left(\langle N\rangle\right.$, atoms per cluster) and density $\left(n_{c}\right)$ are optimized via adjusting the stagnation pressure and temperature of the supersonic nozzle. To illustrate the high effectiveness in density modulation when using clusters, Fig. 1.1 shows an example where we placed a stainless steel grid consisting of five bars directly on the top of the exit of a supersonic nozzle. These bars fabricated with laser cutting have a width of 200 $\mu \mathrm{m}$ and are separated by an equal spacing of $1 \mathrm{~mm}$. We applied a Rayleigh scattering technique to identify the cluster formation and relative density distribution. Spatially 


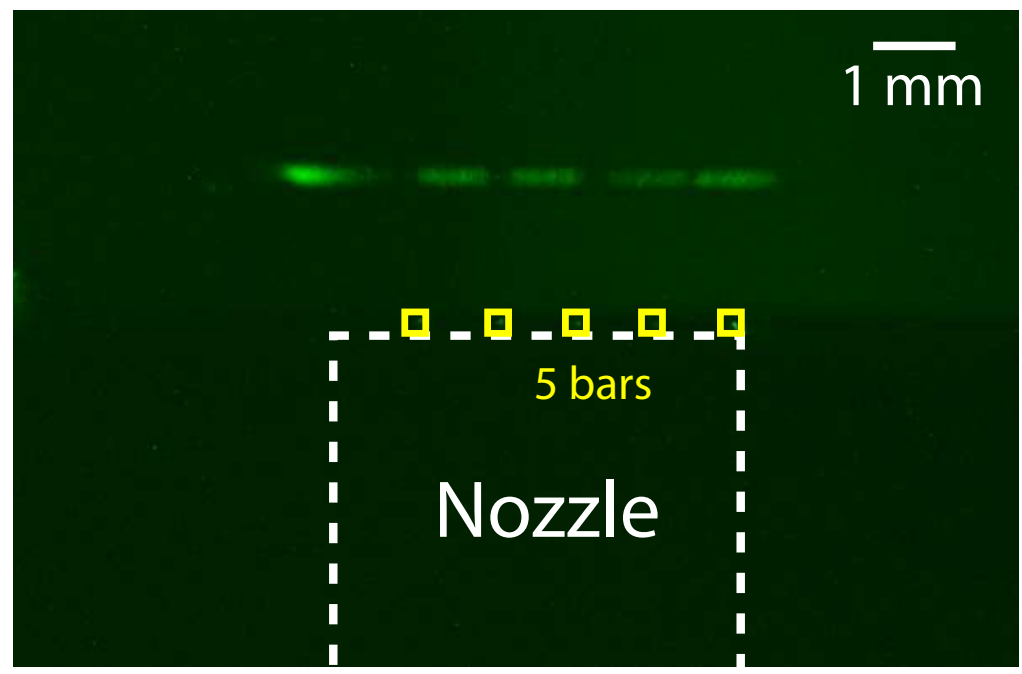

Figure 1.1: Rayleigh scattering image of a density modulated cluster jet measured at around $2 \mathrm{~mm}$ above the exit of the nozzle at a stagnation pressure of 30 bar. The density modulation is achieved by placing a stainless steel grid containing five bars (yellow squares) with a width of $200 \mu \mathrm{m}$ on the top of the nozzle. The spacing between two adjacent bars is set at $1 \mathrm{~mm}$.

modulated scattered signals (five green strips) are observed about $2 \mathrm{~mm}$ above the nozzle, which confirms that a density modulated cluster jet has been generated.

Nevertheless, our experiments using the shown jet with various different modulation periods for HHG only yielded a reduced output as compared to the case without modulation. These initial experiments rather confirmed that using such a density modulated cluster jet for achieving QPM in HHG, even if basically promising, first requires a thorough investigation of the essential ingredients. For instance, we found that an important parameter, the liquid mass fraction, $g$, which characterizes the ratio of the number of atoms in the form of clusters to the total number of atoms in the jet, is generally treated misleadingly in previous research $[47,48,51]$. In those studies, $g$ is usually set equal to unity without any justification, i.e., it is assumed that all atoms leaving the nozzle are part of a cluster (pure cluster jet). In reality, there is an unknown, often even dominating amount of gaseous atoms in the background (gas jet with cluster combination). This, in turn, leads to an overestimation of the average cluster size and density in the jet. Furthermore, due to the background gas, the sharp density contrast behind the obstacles might be spoiled, since, as mentioned above, gaseous atoms diffuse quickly into all directions after passing by obstacles due to their relatively small masses. Researchers have usually estimated the nonlinear response by comparing the $\mathrm{HH}$ yield from cluster jets with a well-known pure gaseous target, e.g., a continuous gas nozzle [48]. However, making wrong assumptions on the liquid mass fraction makes if difficult or impossible to determine the single-atom nonlinear response in clusters, e.g., it is unclear whether atoms bounded in clusters indeed provide a higher nonlinear response than gaseous atoms. However, with a wrong assumption of the amount and sizes of clusters, the interpre- 
tation of the nonlinear response of clusters from experimental measurements remains questionable. Finally, for choosing proper modulation periods in QPM schemes, there is a central aspect which has not been taken into account, namely, the transient nature of QPM, i.e., its variation on an ultrafast time scale due to the rapidly increasing ionization fraction during the drive laser pulse. As a result, it becomes impossible to achieve QPM for any selected harmonic during the entire drive laser pulse with a fixed modulation period. This raises the question of how to choose a proper modulation period for maximizing the total harmonic output pulse energy.

In summary, density modulated cluster jets are holding the fundamental promise of enabling QPM schemes in HHG for increasing the efficiency in generating shorterwavelength radiation. Nevertheless, we have identified three major fundamental issues which need to be clarified before experimental results can be properly interpreted and before QPM can live up to its promise. This thesis aims on an improved understanding of the underlying physics of these three issues and to identify the actual requirements for employing modulated cluster jets for quasi-phase matched high-order harmonic generation.

- First, we note that a trustworthy method to determine the absolute average size of clusters, $\langle N\rangle$, with densities as used in HHG from a supersonic jet expansion is not available. This is an absolutely central shortcoming because it also means that in previous experiments on HHG with clusters other researchers have assumed wrong or unproven cluster properties for data interpretation. Previous studies to determine $\langle N\rangle$ often rely on an experimental power law [52-54], which has been satisfactorily reproduced only in a relatively small range of the so-called Hagena parameter [52], and only for nozzles with a simple conical symmetry. This power law has been frequently assumed to be valid also for experiments which are far beyond the confirmed range of validity. A related problem with unknown cluster properties lies in the correct interpretation of measurements using inteferometric and scattering techniques: to obtain experimental information about $\langle N\rangle$, interferometric techniques are often applied which yield the total atomic number density, $n_{a}$, combined with Rayleigh scattering techniques that measure the product of $\langle N\rangle, n_{a}$ and the liquid mass fraction, $g$. However, in order to obtain $\langle N\rangle$, independent additional information on $g$ is required. Frequently, some unjustified assumptions are made, for instance, by choosing $g=1[51,55]$ which claims without proof that all atoms in the jet have condensed into clusters. However, $g$ is a key parameter in all experiments that attempt to quantify or make use of beneficial properties of clusters, and needs to be accurately determined, since, as mentioned above, it will strongly influence the density contrast in the modulated cluster jet as well as the average cluster size. On the other hand, a modelling approach for deriving $\langle N\rangle$ requires a very complicated nucleation theory for the cluster formation. The predicted values of $\langle N\rangle$ are very sensitive to several parameters such as the surface tension, the liquid mass density, the growth rate and the temperature.

In Chapter 4, we present a comprehensive nucleation theory and investigate all these sensitive parameters in cluster formation during the expansion in a supersonic nozzle. In combination with experimental measurements using Rayleigh scattering and interferometry, we derive the liquid mass fraction and a new ex- 
perimental power law that can be used to determine the average cluster size, $\langle N\rangle$, covering a range from about a thousand to almost ten millions atoms per cluster as is appropriate for HHG.

- Secondly, the basic mechanism as well as the strength of the nonlinear response of atoms in clusters are not well clarified due to the complex structure of clusters. The simple three-step model which describes the HHG in gaseous atoms may fail to explain HHG in clusters. In particular, the recombination process is unclear. For instance, the initially tunnel ionized electron may recollide with its neighbouring ion instead with its parent ion, resulting in the emission of incoherent Bremsstrahlung $[47,56]$. This would extend the cut-off energy beyond the classical cut-off limit for HHG in gas monomers. Others [48, 57] proposed that the harmonic radiation may be generated from the partially delocalized wave function spreading over the whole cluster due to its compact structure. All these interpretations show that HHG from clusters is strongly dependent on the average cluster size, $\langle N\rangle$. The conversion efficiency for HHG in clusters can be lower than that in gas monomers when very large clusters (above 1000 atoms per cluster) are formed, while there are claims that it is higher in rather small clusters consisting of only a few hundred atoms [48]. The according experiments on HHG from clusters assumed for their evaluation of the cluster nonlinearity that the generated radiation would originate exclusively from clusters. However, according to the discussion above, the liquid mass fraction, $g$, plays an important role here. Neglecting the $\mathrm{HH}$ contribution from gas monomers in the measurements may lead to wrong conclusions on the nonlinear response in clusters.

In Chapter 5, we present a detailed experimental study of HHG from a cluster jet over a broad tuning range of the average cluster size, $\langle N\rangle$. In addition to tuning the cluster size via the backing pressure of the jet, the liquid mass fraction was varied by changing the nozzle temperature, while keeping the average cluster size unchanged. In combination with the revisited value of $g$ from the investigation presented in Chapter 4, we obtain what we believe is the first reliable measurement of the single-atom response for clusters vs. gas monomers in HHG.

- Thirdly, in order to apply quasi-phase matching based on density modulated cluster jets for realizing the most efficient HHG, it is required to investigate theoretically what modulation period is providing the maximum output pulse energy. As this modulation period should depend on the coherence lengths in both the inphase and the out-of-phase regions, it is essential to calculate the wave-vector mismatch in both regions. However, the phase-matching condition in HHG is extremely transient due to the time-dependent growth of the ionization fraction during the drive laser pulse. As a result, the wave-vector mismatch in the neutral atoms and plasma varies strongly on an ultrafast time scale. This wave-vector mismatch cannot be compensated during the entire drive laser pulse by applying a fixed modulation period. This raises the question which time interval needs to be chosen within the drive laser pulse for optimizing the HH output pulse energy. The standard suggestion of imposing QPM at the peak intensity of the drive laser pulse, where the highest harmonic dipole amplitude of atoms is usually achieved, 
does not massively correspond to the time-integrated maximized harmonic yield, i.e, the pulse energy.

In Chapter 6, we present the first comprehensive time-dependent model for quasiphase matching in high-order harmonic generation that takes these transient effects on phase matching into account. Using the model we conclude that for optimizing the $\mathrm{HH}$ output pulse energy, QPM has to be imposed in the rising edge of the drive laser pulse.

The described investigations of these fundamental ingredients for quasi-phase matched high-order harmonic generation in clusters may form a solid foundation for further experimental process towards generation of XUV and soft X-ray radiation with high spatial and temporal quality.

\subsection{OUTLINE OF THE THESIS}

This thesis is organized as follows: in Chapter 2, we recall the basic theoretical background needed to understand the formation of atomic clusters, as well as the generation of high-order harmonics. In Chapter 3, detailed technical information is presented on the experimental apparatus constructed for characterizing the cluster formation in a supersonic gas jet and for generating high-order harmonics. In Chapter 4 , we determine the average size of argon clusters generated with a planar nozzle, based on optical measurements in conjunction with theoretical modelling. With a trustworthy value of the liquid mass fraction determined from our nucleation theory, a new power law for predicting the cluster size in experiments is provided. In Chapter 5, an experimental study of HHG in an argon cluster jet produced by a supersonic nozzle is performed under a wide range of stagnation pressures and temperatures. Combined with the liquid mass fraction calculated in Chapter 4, the relative $\mathrm{HH}$ dipole amplitudes for atoms in argon clusters vs. argon as monomers are obtained. We find that the induced HH dipole amplitude of small argon clusters (average cluster size, $\langle N\rangle$, smaller than 1000 atoms per cluster) is strongly size dependent, however, the individual atomic response in a cluster remains the same as that of argon gas monomers. In Chapter 6 we investigate the implications of the highly transient character of phase matching caused by the increasing ionization fraction during the HHG process. A time-dependent QPM model for HHG is developed and rules for the optimum choice of the modulation period are derived that maximize the time-integrated harmonic yield, i,e., the output pulse energy. The maximum pulse energy is achieved when transient quasi-phase matching is provided in the leading edge of the drive laser pulse. In Chapter 7, we conclude the thesis by summarizing the results and suggesting several ideas for improvements and future work. 


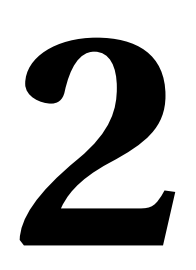

\section{THEORETICAL BACKGROUND OF CLUSTER FORMATION AND HIGH-ORDER HARMONIC GENERATION}

This chapter reviews the background and theory of cluster formation as well as of highorder harmonic generation (HHG), which is essential to understand the characteristics of the clusters, the measurements of high-order harmonic signals and the quasi-phase matching model for HHG presented in this thesis. 


\subsection{Cluster Formation}

Clusters, providing unique properties and novel options for high-intensity laser-matter interactions, are formed via van-der-Waals aggregation of gas atoms or molecules. Because van-der-Waals forces are comparatively weak, such aggregation requires increased pressures and low temperatures as can be achieved, for instance, via supersonic expansion in a nozzle with a given profile mounted in a vacuum chamber. To recall the mechanism of cluster formation in a supersonic jet expansion, we begin in Section 2.1.1 with basic concepts of a gas flow including its thermodynamic properties, and different flow regimes. Next, in Section 2.1.2, we introduce an analytical description of a quasi onedimensional compressible gas flow generated in the nozzle. Finally, we briefly describe our theoretical approach of cluster formation in Section 2.1.3.

\subsubsection{BASIC CONCEPTS OF GAS FLOW}

\section{THERMODYNAMIC PROPERTIES}

In local thermodynamic equilibrium, the basic thermodynamics properties of a given gas with molar mass $M$ can be fully determined by three variables, namely its temperature $T$, pressure $p$, and density $\rho$. The relation among these three variables is given by the equation of state [58]. For an ideal gas, the equation of state can be described by the ideal gas law, with the ideal gas constant $R$, which reads

$$
p=\frac{\rho R T}{M} .
$$

Three assumptions here to be satisfied to address a gas as ideal, such that Eq. (2.1) is a good description. First, the size of the gas atoms or molecules is much smaller than the average distance between different gas particles. Secondly, the gas particles are treated as free particles which do not experience repulsive or attractive forces, and thirdly that, all collisions among particles are elastic. In this thesis, the single gas particles (called monomers) present through the supersonic expansion satisfy the above assumptions and may be treated as ideal gases, while the generated clusters, consisting of hundreds or thousands of particles via van-der-Waals aggregation, immediately fail to satisfy the above assumptions. A detailed description of the thermodynamic properties will be discussed in Chapter 4.

Here, we start with considering an ideal gaseous medium and describe its energy content. The quantity defining the energy content is the internal specific energy, $e$, which can be expressed as a function of the gas temperature

$$
e=\frac{1}{2} f \frac{R T}{M} .
$$

Here, $f$ is the number of degrees of freedom for the gas particles. For a monoatomic gas, $f=3$, while for a diatomic gas another two degrees of freedom associated with rotation around the center of mass of the molecule are taken into account. According to the first principle law of thermodynamics (energy conservation), the internal energy of a system can be increased by adding heat energy, $\delta q$, to or by performing work, $\delta w$, on the system. The change of the internal energy can be mathematically expressed as

$$
\delta e=\delta q+\delta w
$$


in which the exerted work can be expressed via the state variables $p$ and $\rho$ as

$$
\delta w=-p \delta \frac{1}{\rho}
$$

and the added heat can be expressed by a change in entropy, $\delta s$, at constant temperature, $T$, as

$$
\delta q=T \delta s .
$$

When considering the total energy contained in a gaseous medium which is at rest and in thermal equilibrium with its environment, the energy associated with the non-zero volume of the medium has to be taken into account. Namely, to reach a certain thermal equilibrium state from absolute zero total energy, the energy equalling its internal energy $e$, plus the work performed in pushing against the ambient pressure, $p / \rho$, must be applied. Thus, the total energy that can be released by expansion of a gaseous medium with a certain volume is the sum of the internal energy and the work performed in the expansion. This total energy is usually called enthalpy and is defined as

$$
h \equiv e+\frac{p}{\rho} .
$$

Accordingly, if heat is transferred to a medium while it expands, the total energy balance is described by $\delta q$ and can be written as

$$
\delta q=\delta h-\frac{d p}{\rho}
$$

Heat can be transferred to a gaseous medium under different boundary conditions. The two experimentally easiest to realize methods are either maintaining a constant pressure or maintaining a constant volume. By measuring the amount of energy needed to raise the temperature of the gaseous medium by $1 \mathrm{~K}$ one defines the corresponding specific heat capacities, $c_{p}$ and $c_{V}$, as

$$
\begin{aligned}
c_{p} & =\left(\frac{\partial h}{\partial T}\right)_{p}, \\
c_{V} & =\left(\frac{\partial e}{\partial T}\right)_{V} .
\end{aligned}
$$

The difference between $c_{p}$ and $c_{V}$ is only depending on the molar mass, which can be seen by substituting Eq. (2.2) in Eqs. (2.8) and (2.9)

$$
c_{p}-c_{V}=\frac{R}{M}
$$

Similarly, the ratio between the two specific heat capacities, $\gamma$, can be calculated and is called the adiabatic index,

$$
\gamma=\frac{c_{p}}{c_{V}}, \gamma=\frac{f+2}{f}
$$

From the second expression in Eq. (2.11), it is found that the adiabatic index is a universal constant given by the degrees of freedom. For a monoatomic gas $\gamma=5 / 3$ and for 
a diatomic gases, $\gamma=7 / 5$. Since for a gaseous medium, the number of degrees of freedom contributing to the internal energy is constant, except at very high temperatures, the medium can be regarded as what is called a caloric perfect gas, where $c_{p}$ and $c_{V}$ can be treated as constant values. Therefore, the internal energy $e$ and enthalpy $h$ can be expressed as extensively simple functions of temperature, which read

$$
e=c_{V} T
$$

and

$$
h=c_{p} T \text {. }
$$

Under the same conditions, the change in entropy can be calculated straightforwardly as well. If a process brings a gaseous medium from state 1 (with $p=p_{1}, T=T_{1}, \rho=\rho_{1}$ ) to state $2\left(p_{2}, T_{2}, \rho=\rho_{2}\right)$ occurs, the change in entropy $\delta s$ in such a process is given by

$$
\delta s=c_{p} \ln \frac{T_{2}}{T_{1}}-\frac{R}{M} \ln \frac{p_{2}}{p_{1}} .
$$

For isentropic processes, in which the entropy is conserved, one can derive from Eq. (2.14) also a simple relation between the pressure $p$ and the density $\rho$ as

$$
\frac{p}{\rho^{\gamma}}=\text { constant }
$$

Generally, the rapid expansion of gas in a nozzle can be treated as an isentropic process. More strictly, such expansion can also be seen as a reversible adiabatic process. The latter is defined as a process in which no heat is exchanged with the environment. Specifically, the process can be called reversible when no energy is dissipated into the environment as heat [59].

In conclusion so far, we recalled the basic properties that describe the thermodynamic state of an ideal gas and the change of state parameters under certain boundary conditions. In the following, we will make use of these relations and focus on the description of the collective kinetic properties of multiple gas particles (an ensemble of gas atoms) in a flow, such as the continuity and velocity of the flow.

\section{FLOW REGIMES}

To describe the macroscopic kinetic behaviour of a gas, two different approaches are usually considered [60]. One is to model the molecular dynamics with statistical methods, valid when the gas particles can move freely in a certain volume and travel large distances before colliding with other particles. Another approach is to treat the gaseous medium as a continuum flow when the gas particles only travel a rather short distance between collisions. Quantitatively, a dimensionless number, i.e., the Knudsen number [61] $K_{n}$, is used to characterize the flow regimes and determine the proper mathematical approaches. The Knudsen number compares the mean free path of the gas particles, $l$, to a representative physical length scale, $L$, of the system

$$
K_{n} \equiv \frac{l}{L}
$$


For calculating the Knudsen number from the state parameters and physical properties of a gas, assuming a Maxwellian distribution of the particle velocities, the mean free path of the particles is given by

$$
l=\frac{1}{4 \sqrt{2} \pi r_{w}^{2} n},
$$

where $r_{w}$ is the van-der-Waals radius [62], and $n$ is the number density of the particles. With Eq. (2.17), the Knudsen number can be re-written as follows

$$
K_{n}=\frac{R T}{4 \sqrt{2} \pi r_{w}^{2} p N_{A} L},
$$

where $N_{A}$ is the Avogadro constant. As a typical example, if the Knudsen number is below $10^{-3}$, the mean free path of the particles is much smaller than the length scale of the system, and one can treat the gas flow to very good approximation as a continuum flow, which can be characterized by applying Navier-Stokes equations. However, when the Knudsen number grows up to 0.1 , the flow enters the so-called slip flow regime. In this regime, the gas velocity and temperature at the wall of a channel that guides the flow differs significantly from the temperature of the wall itself. Nevertheless, it turns out that the Navier-Stokes equations are still a powerful tool to describe the flow, if the specific conditions at the walls are taken into consideration. When the mean free path of the particles is larger or comparable to the length scale of the system $\left(K_{n}>1\right)$, the gas flow has to be treated as a molecular flow using statistical methods.

For describing the supersonic gas expansion in the nozzle, one has to take into account that the Knudsen number changes its value along the flow trajectories. From Eq. (2.16), it can be seen that the Knudsen number is inversely proportional to the gas pressure. It reaches its maximum at the exit of the nozzle due to a huge pressure drop along the nozzle. To calculate the maximum Knudsen number at this point for a relevant example, we make an estimate for the nozzle used in this thesis (expansion angle of $14^{\circ}$ and an exit diameter of $1 \mathrm{~mm}$ ). Assuming a typical experimental setting, i.e., a reservoir pressure of $P=10 \mathrm{bar}$ and a temperature of $T=300 \mathrm{~K}$ for argon gas, the mean free path of the gas atoms is in the order of $1 \mu \mathrm{m}$ at the nozzle exit, corresponding to a very small value of the maximum Knudsen number of 0.001 . As a result, treating the gas flow throughout the entire nozzle as a continuum flow is well-justified.

\section{SPEED OF SOUND}

Due to the supersonic expansion of the gas, the velocity of the gas flow may reach values far greater than the speed of sound, which is the speed at which an acoustic wave travels through a medium. In a gaseous medium, acoustic waves propagate by small disturbances in pressure. Correspondingly, any information about the system of the gas flow, such as when the flow becomes locally compressed or decompressed by the nozzle geometry or ambient pressure change, can only travel as an acoustic wave to other locations in the flow at the speed of sound. The speed of sound is defined in terms of the pressure change resulting from a change in density while conserving entropy, which can be written as

$$
u_{s}=\sqrt{\left(\frac{\partial p}{\partial \rho}\right)_{s}} .
$$


Combined with Eqs. (2.1) and (2.15), the speed of sound, $u_{s}$, for an ideal gas can be derived as follows

$$
u_{s}=\sqrt{\frac{\gamma R T}{M}}
$$

Eq. (2.20) shows that the speed of sound only depends on the species of gas and the temperature. As an example, we find for argon, that at room temperature ( $300 \mathrm{~K})$, the speed of sound amounts to about $321 \mathrm{~m} / \mathrm{s}$. From a comparison with a kinetic description, one could expect that the speed of sound is of the same order of magnitude as, but limited to, the velocity at which the gas particles travel between collisions. The velocity of the particles can be described by a Maxwellian distribution with an average velocity of

$$
\langle u\rangle=\sqrt{\frac{8 R T}{\pi M}} .
$$

Evaluating Eq. (2.21), for a monoatomic gas where $\gamma=5 / 3$, indeed, the sound velocity and average particle velocity are about the same, the latter being only a factor of about $\sqrt{8 / \gamma \pi} \approx 1.25$-times smaller.

When dealing with changing velocities of the gas flow with the same order of magnitude or even higher than the speed of sound, it is convenient to use a dimensionless number for describing the relative velocity of the gas flow. This number is called Mach number, $M_{a}$, and is defined as the ratio between the velocity of the gas flow $u$ and the speed of sound $u_{s}$

$$
M_{a} \equiv \frac{u}{u_{s}}
$$

A gas flow with $M_{a}<1$ is called subsonic flow, while a gas flow with $M_{a}>1$ is called supersonic flow.

\subsubsection{QUASI ONE-DIMENSIONAL MODEL OF THE GAS FLOW}

In this section, using the concepts and definitions introduced above, we present a simplified description of the dynamics of the gas flow in the nozzle, i.e., where gas condensation (cluster formation) is still neglected. Generally, for most applications, the nozzle is mounted in a vacuum chamber and the flow originates from a gas reservoir that is held under a high pressure, typically ranging from several bars to tens of bars. As is discussed in Section 2.1.1, due to a low Knudsen number, $K_{n}=l / L \ll 1$, the gas particles can collide inside the nozzle yielding a supersonic flow, which can be treated as a compressible continuum flow. In this case, using conservation laws and the shape of the nozzle, the dynamics of the flow in the nozzle can be well described by a quasi one-dimensional model [63].

For the detailed description, we consider a steady, isentropic, quasi one-dimensional flow through a nozzle with varying cross-sectional area $A(x)$, as shown in Fig. 2.1. The gas enters the inlet from the reservoir maintained at so-called stagnation conditions represented by $p_{0}, T_{0}, \rho_{0}$ and $u_{0}=0$. The properties of the gas flow such as temperature $(T)$, pressure $(p)$, density $(\rho)$ and velocity $(u)$, due to expansion and thermodynamic state change and become a function of the position, $x$, along the direction of the flow. 


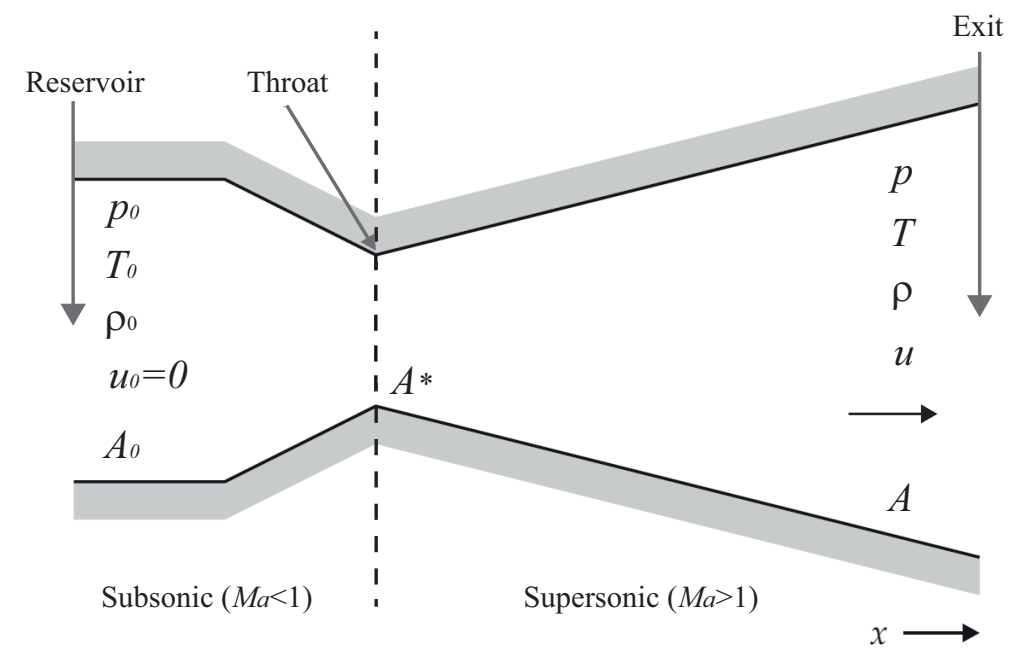

Figure 2.1: Schematic of a quasi one-dimensional gas flow through a nozzle with variable cross-sectional area $A$ (figure adapted and modified with permission from Wolterink [64, 65].

To calculate these properties along the nozzle, three conservation equations are used, namely for mass conservation, Eq. (2.23), for momentum conservation, Eq. (2.24) and for energy conservation equation, Eq. (2.25),

$$
\begin{gathered}
\rho u A=\rho_{0} u_{0} A_{0}, \\
p A+\rho u^{2} A+\int_{A}^{A_{0}} p d A=p_{0} A_{0}+\rho_{0} u_{0}{ }^{2} A_{0}, \\
h+\frac{1}{2} u^{2}=h_{0}+\frac{1}{2} u_{0}{ }^{2} .
\end{gathered}
$$

Using the properties of a calorically perfect gas, described by Eq. (2.8), a relation between the velocity and temperature of the flow can be derived as follows

$$
\frac{T_{0}}{T}=1+\frac{u^{2}}{2 c_{p} T} .
$$

By making use of the Mach number, $M_{a}$, the heat capacity, $c_{p}$, in Eq. (2.26) can be eliminated

$$
\frac{T_{0}}{T}=1+\frac{\gamma-1}{2} M_{a}^{2}
$$

Next, the pressure, $p$, and density, $\rho$, inside the nozzle can be similarly calculated by using Eq. (2.15) as follows:

$$
\begin{aligned}
& \frac{p_{0}}{p}=\left(1+\frac{\gamma-1}{2} M_{a}^{2}\right)^{\frac{\gamma}{\gamma-1}}, \\
& \frac{\rho_{0}}{\rho}=\left(1+\frac{\gamma-1}{2} M_{a}^{2}\right)^{\frac{1}{\gamma-1}} .
\end{aligned}
$$


Eqs. (2.27), (2.28), (2.29) are the three basic equations for describing the gas flow in the nozzle. These equations state that, once the Mach number along the direction of flow is known $\left(M_{a}(x)\right)$, the gas temperature $(T(x))$, pressure $(p(x))$ and density $(\rho(x))$ become known along the expansion axis $x$ as well, only dependent on the stagnation conditions $T_{0}$ and $p_{0}$ in the reservoir. To determine the Mach number of the gas flow inside the nozzle, we compare the gas density in the flow to the gas density at the position where flow reaches the speed of sound, $M_{a}=1$. By combining Eq. (2.23), Eq. (2.25) and Eq. (2.29), we obtain the relation between the cross-sectional area, $A$, and the Mach number, which reads

$$
\frac{A}{A_{*}}=\frac{1}{M_{a}}\left(\frac{2}{\gamma+1}\left(1+\frac{\gamma-1}{2} M_{a}^{2}\right)\right)^{\frac{1}{2} \frac{\gamma+1}{\gamma-1}},
$$

where $A_{*}$ is the cross-sectional area of the nozzle at $x=x_{*}$ where the speed of sound is reached. Eq. (2.30) indicates that the Mach number only depends on the relative crosssectional area. The area $A_{*}$ can be found by differentiating Eq. (2.23) with respect to $x$ and eliminating the density term. The resulting equation relates a change in the flow velocity, $\delta u$, to a change in cross-sectional area $\delta A$, which reads

$$
\frac{\delta u}{u}=-\frac{1}{1-M_{a}^{2}} \frac{\delta A}{A} .
$$

From Eq. (2.31), it can be seen that the flow velocity reaches the sound velocity at $\delta A=$ 0 , i.e., exactly at the throat of the nozzle, which is the location of the minimum crosssectional area. Therefore, the dynamics of the gas flow inside the nozzle illustrated in Fig. 2.1 can be summarized as follows: the gas flow is first compressed to accelerate it towards the speed of sound $\left(0<M_{a}<1\right)$ from its initial speed of zero $\left(M_{a}=0\right)$ in the reservoir. The speed of sound $\left(M_{a}=1\right)$ is reached at the minimal cross-section of the nozzle, namely the throat of the nozzle. Afterwards the cross-sectional area is increasing again and the flow $\left(M_{a}>1\right)$ expands in the diverging section which accelerates the flow further to supersonic speeds.

In the quasi one-dimensional flow discussed above, the Mach number keeps increasing throughout the nozzle and becomes larger than unity due to the diverging shape of the nozzle. Inserting the increasing Mach number in Eqs. (2.27), (2.28) and (2.29) shows that a significant drop of the temperature, pressure and density of the gas occurs along the direction of the flow during the expansion as long as the velocity keeps increasing. However, there is a fundamental limit to the maximum velocity of a gas flow during expansion. Considering energy conservation (Eq. (2.25)), it can be shown that the maximum possible velocity is achieved when all initial enthalpy is converted into kinetic energy. Thus, the maximum velocity can be expressed as [64]

$$
u_{\text {max }}=\sqrt{\frac{2 R \gamma}{M(\gamma-1)} T_{0}} .
$$

For instance, with the reservoir at room temperature, $T_{0}=300 \mathrm{~K}$, the maximum velocity of an argon flow amounts to about $560 \mathrm{~m} / \mathrm{s}$. Important for our experiments is that, due to a sufficiently deep drop of the temperature that can be gained in a supersonic gas flow (and not readily in a subsonic flow), single gas particles may condense and form 
so-called clusters. It can be expected that the size and volume density of such clusters depend strongly on the temperature and pressure dynamics in the nozzle, which can be set via the temperature and pressure in the reservoir as well as via the shape of the nozzle.

\subsubsection{THEORETICAL APPROACH TO CLUSTER FORMATION}

As mentioned in the above section, clusters are often formed in a supersonic expansion that, for instance, can be realized by expanding gas from a reservoir at high pressure and low temperature through a nozzle into vacuum [54]. The gas atoms or molecules forming clusters aggregate via van-der-Waals forces. Qualitatively, the formation of clusters (condensation of gas particles) can be illustrated in a so-called P-T-phase diagram as will be described in Chapter 4. However, a quantitative prediction of cluster formation is extremely difficult. During the last decades, a large number of models have been developed, especially nucleation theories [66]. These models can be divided into three different categories, namely classical nucleation theory first proposed by Becker and Döring [67], mean-field kinetic nucleation theory, first proposed by Kalikmanov [68], and extended modified liquid drop model-dynamical nucleation theory, first proposed by Reguera and Reiss [69]. Further attempts to predict cluster formation include various numerical simulations such as Monte Carlo-type of calculations, molecular dynamics and density functional theory [70-72]. Unfortunately, these models are extremely complicated and require vast computational efforts. Up till now, there is no rigorous model available that successfully, i.e., quantitatively, correctly describes the entire cluster formation process for all substances or conditions. Several important parameters, such as the thermodynamic properties of clusters as well as the nucleation and growth rate of clusters, are not yet quantified accurately. A small variation of these parameters will result in huge deviation (several orders of magnitude) of the characteristics of the cluster such as the size and density distribution.

\section{HAGENA'S SCALING LAW}

Nevertheless, with the development of semi-empirical scaling laws by Hagena [52, 73], within certain limited parameter ranges, the process of cluster formation in a supersonic expansion can be approximately described. Following theoretical considerations as well as numerous experimental observations on gas condensation in different supersonic expansions from different conical nozzles, a dimensionless scaling parameter, the so-called Hagena parameter, $\Gamma^{*}$, has been defined to describe the cluster formation:

$$
\Gamma^{*}=k_{h} d^{0.85} p_{0} T_{0}^{-2.29} .
$$

In this heuristic relation, basically identified via fits to measurements under different experimental conditions, $d$ is the diameter of a round sonic nozzle (in $\mu \mathrm{m}$ ). For a sonic nozzle, the smallest cross-sectional area is located at the exit of the nozzle, where the speed of sound can be reached. $k_{h}$ is a gas-specific constant (for argon, $k_{h}=1650$, for neon, $k_{h}=185$, for helium, $k_{h}=3.85$ ). $T_{0}$ and $p_{0}$ are the reservoir temperature (in K) and the pressure (in mbar), respectively. To extend this equation to the supersonic expansion, an equivalent diameter $d^{*}$ is used, which for a round nozzle (rotationally sym- 
metric) reads

$$
d^{*}=\frac{0.74 d}{\tan \alpha}
$$

where $\alpha$ is the expansion half-angle of the diverging section of the nozzle. Experiments such as time-of-flight mass spectroscopy reveal that no clustering is observed for $\Gamma^{*}<$ 200. At sufficiently high values of $\Gamma^{*}$, massive condensation sets in and the average cluster size, $\langle N\rangle$, was found in experiments to follow approximately a function of $\Gamma^{*}[73]$

$$
\langle N\rangle=33\left(\frac{\Gamma^{*}}{1000}\right)^{2.35},
$$

for $\Gamma^{*}$ ranging from $1000<\Gamma^{*}<7300$. This semi-empirical scaling law is rather useful under certain specific conditions (e.g., for a round sonic nozzle), however it apparently has a number of severe limitations. First of all, it is only valid for the axisymmetric nozzles, while in optical applications with an extended interaction length a planar (slit) nozzle is preferred for confining the transverse density distribution of clusters to one dimension. Such planar nozzles are used particularly in high-intensity laser-matter interaction, such as high-order harmonic generation described in this thesis, requiring sufficiently long interaction lengths. Secondly, due to the limited validity range of $\Gamma^{*}$, the average cluster size can only be determined within a narrow range of temperatures and pressures in the reservoir. For instance, when larger clusters are to be generated, Eq. (2.35) may overestimate the average cluster size [74], which can lead to wrong conditions in optical experiments. Finally and importantly, the scaling law does not predict anything about the fraction of particles that becomes clustered in a supersonic expansion as compared to the total amount of gas particles. This fraction is called liquid mass fraction and is essential and critical to take into account in optical experiments.

In Chapter 4 of this thesis, we present the first complete theory for determining the cluster size that consistently describes cluster formation in supersonic gas jets including a prediction of the liquid mass fraction, and that is valid beyond the range of Eq. (2.35). Specifically, we develop a comprehensive nucleation theory based on the classical nucleation theory mentioned above and investigate all the sensitive parameters in cluster formation during the expansion in a supersonic nozzle, such as surface tension, liquid mass density, growth rate and temperature. In combination with experimental measurements using Rayleigh scattering and interferometry, we derive the liquid mass fraction and a new experimental power law that can be used to determine the average cluster size from Hagena's parameter, $\Gamma^{*}$, in a wider range from $10^{4}$ to $10^{5}$, which is well beyond the proven validity of Hagena's scaling law. The detailed description of the model and results will be presented in Chapter 4 . 


\subsection{High-ORDER HARMONIC GENERATION}

High-order harmonic generation (HHG) is an extremely nonlinear optical process that originates from the nonlinear interaction between high-intensity laser pulses and matter, typically consisting of a gaseous medium. During the interaction, the frequency of a part of the drive laser radiation is up-converted by a relatively large integer number, so-called high-order harmonics $(\mathrm{HH})$ of the original laser frequency, reaching into the extreme ultraviolet (XUV) and soft x-ray range. We begin in Section 2.2.1 with describing the basic principles of HHG process within the semi-classical three-step model which describes the single-atom response to an intense laser field. With this model, several central features of the $\mathrm{HH}$ spectrum can be well understood, such as the overall shape of the spectrum and the spectral location of the cut-off energy region. In Section 2.2.2, we show how the generation efficiency and spectrum of high-order harmonics are influenced by macroscopic properties of the nonlinear medium, where phase matching plays a central role. More specifically, to efficiently generate high-order harmonics, a careful control of the wave-vector mismatch between the drive laser field and the generated harmonic field along the propagation direction is required. The discussion includes also other macroscopic properties of the medium, specifically limitations to efficient HHG through reabsorption of the generated harmonics in the generating medium itself, and limitations in the cut-off wavelength on the spectrum due to ionization. Lastly, we briefly recall the technique of quasi-phase matching (QPM), because QPM is often named as a promising solution for phase matching when generating very high-order harmonics. However, we also discuss the shortcomings of QPM as it is typically assumed to work, as the basis for an extended modelling of dynamic QPM in HHG to be presented in Chapter 6.

\subsubsection{SINGLE-ATOM RESPONSE}

Despite the fact that HHG is a complex optical nonlinear process, which requires a full quantum mechanical description for the signle-atom response of the nonlinear medium [27], it can be quite accurately explained using a simplified semi-classical threestep model introduced by Corkum et al. [26]. The three steps in the description are shown in Fig. 2.2. In brief the three steps involve the following processes:

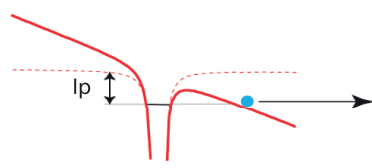

Step I: Ionization

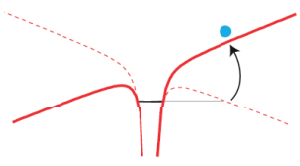

Step II: Propagation

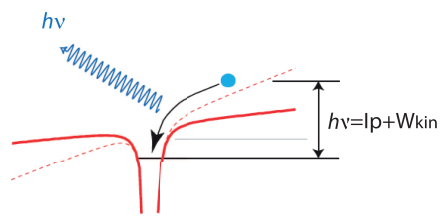

Step III: Recombination

Figure 2.2: Three-step model illustrating the single-atom response in HHG as a sequence of electron tunnel ionization, propagation and recombination. The photon energy $h v$, emitted in the recombination of the electron to its parent ion, equals the sum of the ionization potential of the atom and the kinetic energy of the electron (figure adapted with permission from Winterfeldt et al. [75]. Copyrighted by the American Physical Society). 
I. Ionization Upon the presence of a strong light field, the nuclear binding potential for electrons becomes strongly distorted by adding a potential gradient. An earlier bound electron now leaves the atom via tunnel ionization.

II. Propagation This freed electron is first driven away from its parent ion by the strong electric field and is accelerated. After the electric field has reversed, the electron is driven back towards its parent ion.

III. Recombination The electron recombines in an inelastic collision with its parent ion and emits a high energy photon.

\section{STEP I: IONIZATION}

When an atom is subject to a high-intensity laser field, usually in the form of an ultrashort pulse, a bound electron can be set free from the atomic potential, which is generally called ionization. The ionization can be classified into three different regimes, depending on the strength of the external electric field: (i) multi-photon ionization (MPI), (ii) tunnel ionization and (iii) barrier-suppressed ionization (BSI). Multi-photon ionization (MPI) describes the situation when a relatively low laser intensity is used such that a high number of optical cycles is required for ionization, often supported by atomic resonances to free an electron. Due to the lower-intensity approximation, MPI can be well described by perturbation theory in which the power of the generated harmonics decreases steadily towards high orders [76]. However, when the field strength of the laser pulse becomes comparable to that of the intra-atomic field, such that the atomic Coulomb potential is strongly distorted by the laser field, a valence electron can tunnel through the combined atomic potential within a time interval that is shorter than an optical half-cycle. This process is called tunnel ionization and is essential for HHG. Further, when the field strength of the laser pulse becomes even stronger than that of the intra-atomic field, it can fully suppress the potential barrier. In this case, the electron escapes from the parent ion with tunnelling, i.e., the probability to find the electron still bound after half of an optical cycle is close to zero. This process is named over-thebarrier-ionization. The Keldysh parameter [77], $\gamma$, is a coarse but convenient parameter to distinguish the regimes discussed above, and is given by

$$
\gamma=\sqrt{\frac{I_{p}}{2 U_{p}}}
$$

where $I_{p}$ is the ionization potential of the atom and $U_{p}$ is the ponderomotive energy. The ponderomotive energy, $U_{p}$, is also called quiver energy of the electron in the laser field and can be expressed as

$$
U_{p}=\frac{e^{2} E_{0}^{2}}{4 m_{e} \omega^{2}} \approx 9.3 \times 10^{-14} I\left[\frac{\mathrm{W}}{\mathrm{cm}^{2}}\right] \lambda^{2}[\mu \mathrm{m}],
$$

where $e$ and $m_{e}$ are the charge and the mass of the electron, $E_{0}$ is the strength of the drive laser field with the angular frequency $\omega$. It can be seen that $U_{p}$ is proportional to the drive laser intensity, $I$, and to the square of the wavelength of the drive laser, $\lambda$. 
For $\gamma \gg 1$, multi-photon ionization dominates the ionization process, while for $\gamma \ll 1$, ionization enters the tunneling regime. Considering a typical experimental condition achieved in the experiments for this thesis: HHG in argon $\left(\lambda \approx 800 \mathrm{~nm}, I \approx 10^{14} \mathrm{~W} / \mathrm{cm}^{2}\right.$, $I_{p}=15.6 \mathrm{eV}$ ). In this case, the Keldysh parameter $\gamma=0.93$, which means that tunnel ionization is dominating the ionization process.

Considering the detailed electronic structure in atoms, the ionization rate obtained with tunnel ionization, $\omega_{\mathrm{ADK}}$, has been calculated (in atomic units) by Ammosov, Delone, and Krainov as follows [78]

$$
\omega_{\mathrm{ADK}}(t)=\left|C_{n^{*} l^{*}}\right|^{2} f(l, m) \sqrt{\frac{3 E(t)}{\pi\left(2 I_{p}\right)^{\frac{3}{2}}}} I_{p}\left(\frac{2\left(2 I_{p}\right)^{\frac{3}{2}}}{E(t)}\right)^{2 n^{*}-|m|-1} \exp \left(-\frac{2\left(2 I_{p}\right)^{\frac{3}{2}}}{3 E(t)}\right) .
$$

Here, $E(t)$ is the instantaneous electric field of the laser pulse, $l$ and $m$ are the quantum number of angular momentum and the magnetic quantum number, respectively. The effective principal quantum number $n^{*}=Z\left(2 I_{p}\right)^{-1 / 2}$, as well as the effective orbital quantum number $l^{*}$, includes the ion charge after ionization, $Z$. The constant $\left|C_{n^{*} l^{*}}\right|^{2}$ and the factor $f(l, m)$ are given by

$$
\left|C_{n^{*}} l^{*}\right|^{2}=\frac{2^{2 n^{*}}}{n^{*} \Gamma\left(n^{*}+l^{*}+1\right) \Gamma\left(n^{*}-l^{*}\right)},
$$

and

$$
f(l, m)=\frac{(2 l+1)(l+|m|) !}{2^{|m|}(|m|) !(l-|m|) !} .
$$

Using the ionization rate, $\omega_{\mathrm{ADK}}$, the ionization fraction, $\eta(t)$, defined as the ratio between the density of free electrons, $n_{e}(t)$, and the initial density of the neutral atoms, $n_{0}$, can be calculated as

$$
\eta(t)=\frac{n_{e}(t)}{n_{0}}=1-\exp \left(-\int_{-\infty}^{t} \omega_{\mathrm{ADK}}\left(t^{\prime}\right) d t^{\prime}\right) .
$$

Further increasing the strength of the drive laser pulse field such that the tunnel barrier is largely suppressed, a transition occurs from the tunnel-ionization regime to barriersuppressed ionization. The threshold intensity for BSI is given by

$$
I_{B S}=4.02 \times 10^{9}\left(\frac{I_{p}^{4}}{Z^{2}}\right)
$$

\section{SteP II: Propagation}

Driven by the electric field, $E(t)$, of the laser pulse, the ionized electron propagates following Newton's second law of motion. Assuming that the applied laser field is linearly polarized, that the instantaneous field follows a sinusoidal oscillation, $E(t)=\mathrm{E}_{0} \cos (\omega \mathrm{t})$, and neglecting the potential of the ion core, the equation of motion for the electron can be approximated as

$$
m_{e} \frac{d^{2} x(t)}{d t^{2}}=-e E(t)=-E_{0} \cos (\omega t)
$$

where $x(t)$ is the distance coordinate of the electron from the ion, $E_{0}$ is the amplitude of the electric field and $\omega$ is the laser angular frequency. Assuming the initial position and 
velocity of the electron to be zero at the time of ionization, $t_{0}$, (the corresponding phase of the laser field being $\left.\phi_{0}=\omega t_{0}\right)$, the velocity, $v(t)$, and the position, $x(t)$, of the electron can be derived as

$$
\begin{gathered}
v(t)=-\frac{e E_{0}}{m_{e} \omega}\left[\sin (\omega t)-\sin \left(\omega t_{0}\right)\right], \\
x(t)=\frac{e E_{0}}{m_{e} \omega^{2}}\left[\cos (\omega t)-\cos \left(\omega t_{0}\right)\right]+\frac{e E_{0}}{m_{e} \omega} \sin \left(\omega t_{0}\right)\left(t-t_{0}\right) .
\end{gathered}
$$

Depending on the actual time of ionization, $t_{0}$, and the corresponding laser phase, $\phi_{0}=$ $\omega t_{0}$, the electron can follow several trajectories. Fig. 2.3 shows several calculated trajec-

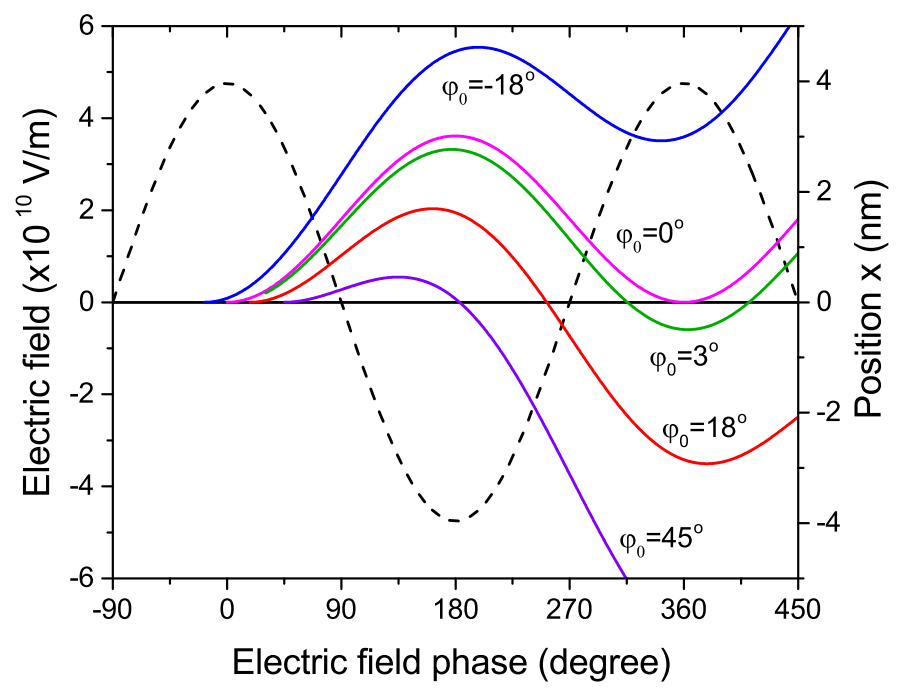

Figure 2.3: Calculated trajectories for the electron ionized at different initial phases, $\phi_{0}$, as a function of the phase of the electric field. The blue line indicates an electron that does not return to its parent ion. The pink line shows an electron returning with zero kinetic energy, while the red line indicates an electron returning with the highest kinetic energy. The green and purple lines represent two different trajectories (short and long), leading to the same final kinetic energy.

tories obtained for different ionization times. The electric field is plotted as a dashed line for reference. Electrons that are ionized before the field maximum (i.e. $-90^{\circ}<\phi_{0}<0^{\circ}$, blue line) will not return to their parent ions, while electrons that are freed in the phase interval $0^{\circ}<\phi_{0}<90^{\circ}$ will be accelerated back towards their parent ions. Ionization at the field maximum leads to the longest trajectory $\left(\phi_{0}=0^{\circ}\right.$, pink line), however, with this particular trajectory, the kinetic energy is zero when the electron returns to the location of the ion $(x=0)$. The highest kinetic energy upon return, resulting in the radiation with the shortest wavelengths near cut-off, is generated at $\phi_{0} \approx 18^{\circ}$ (red line) and its trajectory is referred to as the cut-off trajectory. Besides these two extreme cases with minimum and maximum return energy, the electron trajectories can be classified into two 
groups: long trajectory and short trajectory. The former refers to electrons ionized later, at $18^{\circ}<\phi_{0}<90^{\circ}$ and these return after a short time. The latter refers to the electron ionized earlier, at $0^{\circ}<\phi_{0}<18^{\circ}$, which return after a longer time. These trajectories are experienced in the course of one optical period, which causes a strong intensity dependence of the phase of the short-wavelength radiation [79]. Despite of the different return times for different trajectories, there are always two trajectories (i.e. a pair: a long and a short one, e.g., green and purple curves in Fig. 2.3) that possess the same kinetic energy below $3.17 \mathrm{U}_{\mathrm{p}}$.

\section{STEP III: RECOMBINATION}

When the accelerated electron returns to its parent ion and recombines to re-fill the ground state, a high-energy photon can be emitted carrying any excess energy gained by the electron in the laser field. The energy of the emitted photon can be expressed as

$$
h v=W_{k i n}+I_{p}
$$

where $W_{k i n}$ is the kinetic energy of the accelerated electron when it returns to the ion. As was discussed before, the kinetic energy is strongly dependent on the ionization time, $t_{0}$ (or the phase $\phi_{0}$ ), of the electron and can be calculated as a function of phase $\phi_{0}$ by solving Eq. (2.45) for $x(t)=0$. Fig. 2.4 shows the calculated kinetic energy of an

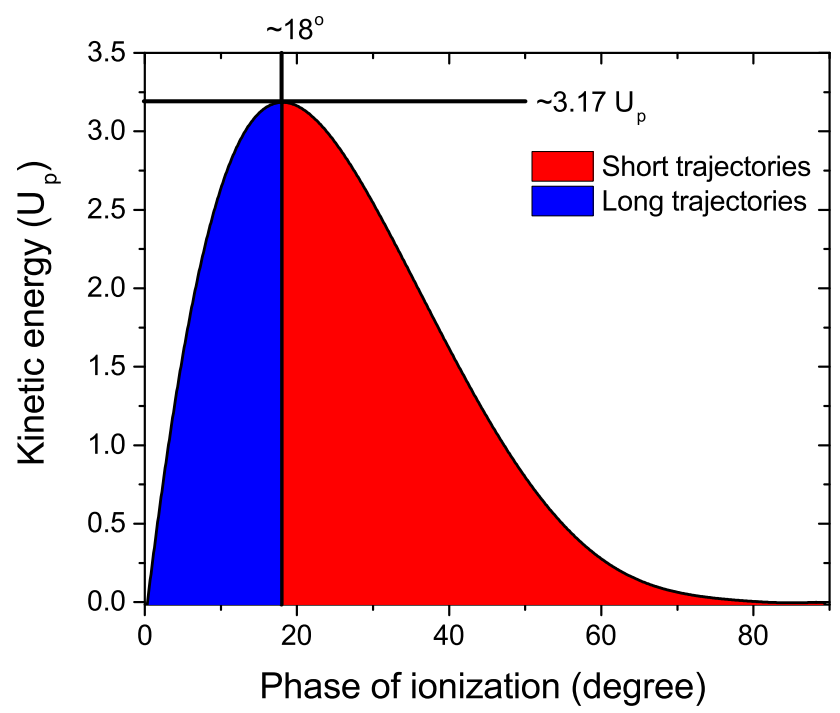

Figure 2.4: Calculated kinetic energy of an electron when it returns to its parent ion as a function of phase, $\phi_{0}$, of the laser field at the moment when the electron is ionized.

electron following different trajectories (red for the short one and blue for the long one) as a function of phase, $\phi_{0}$, of the laser field at the moment when the electron is ionized. The maximum kinetic energy is obtained for a phase $\phi_{0} \approx 18^{\circ}$, corresponding to $W_{\text {kin }} \approx$ 
$3.17 U_{p}$. Consequently, the highest emitted photon energy for HHG [26], called cut-off energy $E_{\text {cutoff }}$, is given by

$$
E_{\text {cutoff }}=h v_{\max }=I_{p}+3.17 U_{p} .
$$

This theoretical value of the cut-off energy matches well with the cut-off measured in the spectrum of previous experiments [80, 81]. A typical spectrum of HHG generated in gaseous media is schematically presented in Fig. 2.5. The spectral amplitude for the low-order harmonics decreases rapidly with increasing harmonic order in the perturbative regime. In the next region, called the plateau, the harmonic intensities remain approximately constant before again dropping significantly at the cut-off.

Another general feature in HH output spectra can be explained by considering the temporal periodicity and spatial symmetry in HHG: according to the three-step model, the recombination process is repeated every half-cycle of the electric field of the laser pulse, i.e., twice per optical cycle. Each time, a short burst or pulse of high energy radiation (XUV or soft X-ray) is generated, leading to a pulse consisting of high-energy photons separated in time by half the optical cycle of the driving laser. This corresponds to a doubled periodicity, which is responsible for the observation of the spacing of two neighbouring harmonics equalling $2 \omega$ in the spectral domain. In addition, when considering the spatial symmetry of the situation in Fig. 2.2, where consecutive bursts are a consequence of recombination from alternating directions, the corresponding spectral components have the same amplitude but opposite phases. This results via superposition over many optical cycles in destructive spectral interference for even order harmonics $\left(\omega_{\mathrm{HH}}=2 m \omega\right)$ and constructive interference $\left(\left(\omega_{\mathrm{HH}}=(2 m+1) \omega\right)\right)$ for odd order harmonics. These arguments explain why, normally, only odd harmonics are observed in measured HH spectra as is shown in Fig. 2.5.

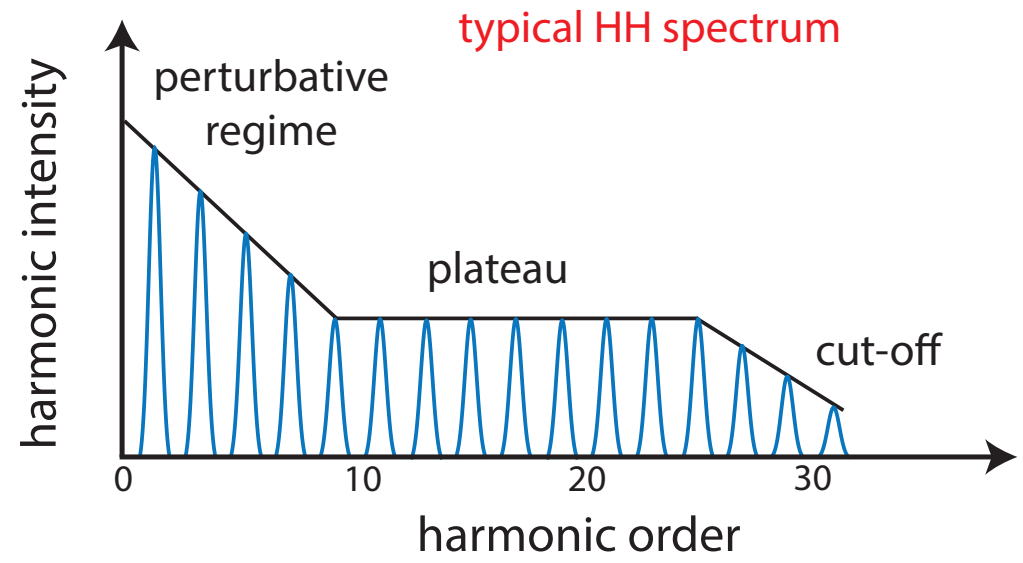

Figure 2.5: Typical spectrum of HHG which can be divided into three regimes: the perturbative regime at low orders, the plateau for intermediate orders and the cut-off at the highest orders.

Moreover, the three-step model also predicts the polarization of the emitted harmonic radiation, which is strongly correlated to the drive laser field. Normally, only a 
linearly polarized drive laser field is suitable for efficient HHG since any slight ellipticity of the drive laser polarization, specifically the transverse component in such field, will drive the electron transversely away from its parent ion, preventing the occurrence of recombination process. The according sharp drop of efficiency for HHG due to the drive field being not linearly polarized has been observed in previous experiments, e.g., by Budil et al. [82].

To understand the HHG process more precisely, a fully quantum mechanical theory has been developed with strong field approximation by Lewenstein et al. [27]. The theory indicates that an electron is promoted into a continuum state by the laser field, then propagates in the laser field freely and finally recombines with the parent ion via a dipole transition. Apart from the conclusions drawn from the three-step model, the fully quantum mechanical theory also predicts the intrinsic phase of the generated harmonic radiation, which is accumulated by the electron as it freely propagates following either short or long trajectories, and which strongly depends on the drive laser intensity [79]. It should also be noted that in such quantum mechanical theory, the presence of alternative classical trajectories is described as a superposition of probability amplitudes for these possibilities, and that the wave packet describing the electron position and momentum spreads out by diffraction. Furthermore, the theory also predicts a slightly higher cut-off energy $\left(E_{\text {cutoff }}=F\left(I_{p} / U_{p}\right) I_{p}+3.17 U_{p}\right)$ than that in the three-step model. The factor $F\left(I_{p} / U_{p}\right)$ is equal to 1.3 for $I_{p} \ll U_{p}$ and approaches unity as $I_{p}$ grows [27].

So far in this chapter, HHG has only been treated as the response of single atoms, or as the so-called microscopic response, which is helpful to understand essential features of the HHG spectrum such as the cut-off energy. However, HHG is usually carried out in samples that contain large ensembles of atoms, as a multi-particle process where also the collective effects have to be taken into consideration. Real experimental observations such as the spectral shape and cut-off of the output are always strongly affected by such collective effects, also called macroscopic, or propagation effects such as phase matching and reabsorption in extended media. Due to their general importance and in view of the calculations presented in this thesis, these macroscopic effects in HHG will be discussed in the next section.

\subsubsection{MACROSCOPIC RESPONSE}

PHASE MATCHING

Before discussing how to realize a macroscopic build-up of desired HHG signals along the generating medium sufficiently strong as measurable harmonic yield, we recall a simple low-order optical parametric process, namely second harmonic generation (SHG) in an optical nonlinear crystal. To generate an output with non-vanishing efficiency, the second harmonic fields generated at different locations in entire medium have to be generated in phase and add up constructively, which is known as phase matching. The phase difference between the fundamental light field at frequency $\omega$ (that drives the radiating nonlinear polarization) and the harmonic light field at frequency $2 \omega$ can be characterized by the wave-vector mismatch, $\Delta k$, where

$$
\Delta k=2 k_{\omega}-k_{2 \omega}
$$


phase matching is achieved, when $\Delta k$ is brought to zero. In the presence of a non-zero $\Delta k$, the frequency conversion is much reduced. By applying perturbation theory [83], where the induced nonlinear polarization is quadratically dependent on the applied field, $P_{N L}=\epsilon_{0} d_{\mathrm{eff}} E^{2}(t)$ and by assuming that the interacting waves have plane phase fronts, the second harmonic intensity generated over the length of the crystal, $L$, can be expressed as a function of the wave-vector mismatch as

$$
I_{2 \omega} \propto d_{\mathrm{eff}}^{2} I_{\omega} L^{2} \operatorname{sinc}^{2}\left(\frac{\Delta k L}{2}\right),
$$

where $d_{\text {eff }}$ is the effective nonlinear susceptibility of the crystal material. The normalized second harmonic intensity is shown in Fig. 2.6 (left). It can be seen that the output decreases quickly from its maximum at $\Delta k=0$ (phase matching), to zero in the form of an oscillating (sinc ${ }^{2}$-shaped) decay for increasing $|\Delta k|$. Similarly, when calculating
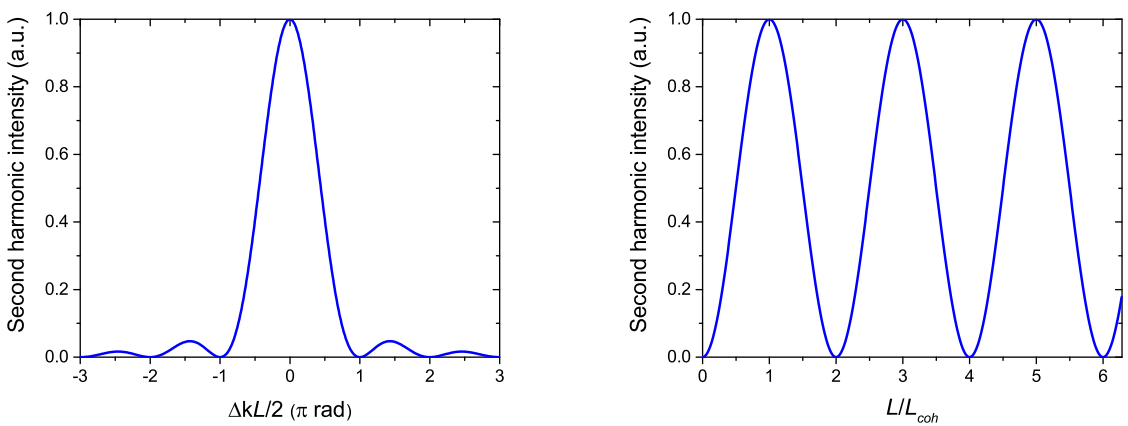

Figure 2.6: Normalized intensity of a second harmonic field as a function of $\Delta k$ for a fixed crystal length $L$ (left) and a function of crystal length $L$ (right).

the second harmonic output vs the crystal length (i.e., vs. propagation distance) for a given nonzero mismatch, $|\Delta k| \neq 0$, Fig. 2.6 (right) shows that the output oscillates in a $\sin ^{2}$-shaped fashion. This oscillation can be explained, e.g., with a phase diagram for the electric field added per infinitesimal propagation step, $d L$, as a repeated coherent growth within the so-called coherence length , $L_{c o h}=\pi /|\Delta k|$, followed by back conversion within the coherence length. In a nonlinear crystal when phase matching is absent $(|\Delta k| \neq 0)$, due to the typical refractive index dispersion in the infrared and visible range, the coherence length is typically in the order of a few micrometers for the optical nonlinear crystal. To more detail, achieving phase matching in SHG requires that the fundamental and second harmonic waves propagate at the same phase velocity. Due to the presence of dispersion, however, it is usually difficult to meet this requirement. A solution to this problem is to use a birefringent nonlinear crystal such as BBO, LBO and KTP. Here the refractive indices can be the same for different light frequencies by using different linear polarizations of the involved light waves. Additionally, the index for light provided along the extraordinary axis can be varied with the propagation direction (rotating the crystal) for SHG in nonlinear birefringent crystals. With such methods, phase matching can generally be achieved. For example, fundamental light traveling as the 
ordinary wave with a central wavelength of $800 \mathrm{~nm}$ can be efficiently converted to the second harmonic in a KDP crystal, if it propagates at an angle of $44.9^{\circ}$ to the optical axis. In this case, the second harmonic radiation propagates as the extraordinary wave [83].

In general, the above discussion can be applied analogously for all other optical parametric processes, including HHG. As is depicted in Fig. 2.7, to achieve efficient HHG in the generating volume, the $\mathrm{HH}$ field contributions generated at different positions (black emitters) in the medium need to add up constructively. This requires the drive laser field to have the same velocity as the generated harmonic field along the propagation direction. This means that, for a given harmonic order, $q$, the wave vector $\Delta k$ can be expressed

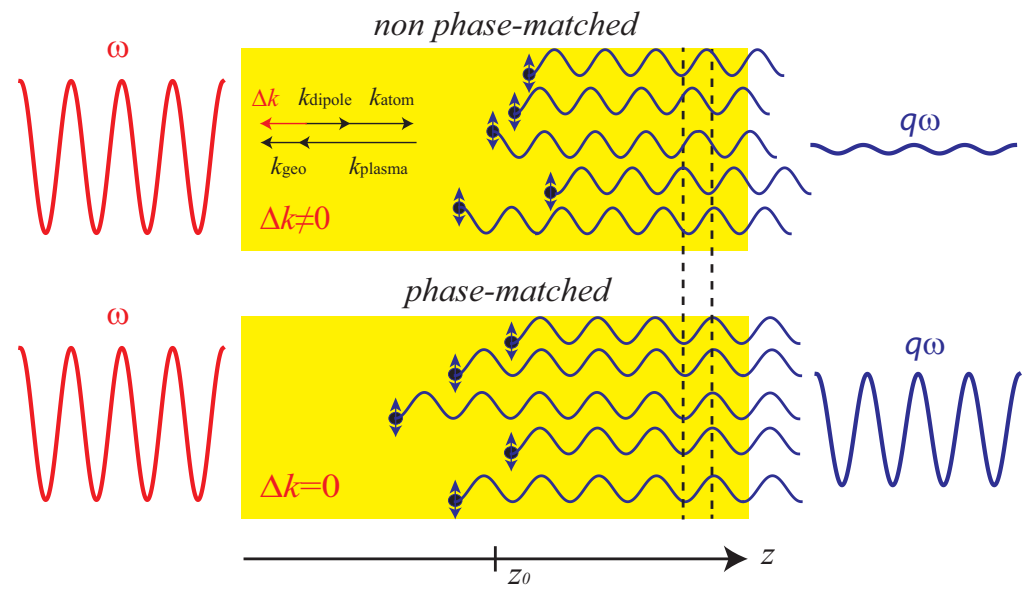

Figure 2.7: Schematic of two different conditions: the absence of phase matching (top, $\Delta k \neq 0$ ) and phase matching (bottom, $\Delta k=0$ ). Red sinusoidal wave is the drive laser field at frequency of $\omega$, while the blue sinusoidal wave is the generated $\mathrm{HH}$ field at frequency of $q \omega$. The black dots represent the induced $\mathrm{HH}$ dipoles emitted at different locations inside the generating medium (yellow area). The black dash lines illustrate the phase front of the generated $\mathrm{HH}$ field. The wave-vector mismatch consisting of four terms $\left(\Delta k_{\text {atom }}, \Delta k_{\text {plasma }}, \Delta k_{\text {geo }}\right.$ and $\left.\Delta k_{\text {dipole }}\right)$ are also indicated.

as $^{1}$

$$
\Delta k=q k_{\omega}-k_{q \omega},
$$

and that $\Delta k=0$ is to be achieved. Here $k_{q \omega}$ denotes the wave vector of the induced polarization at frequency $q \omega$. However, high-order harmonics are usually generated in gaseous media, which are not birefringent but isotropic, such that the phase velocities (refractive index) do not depend on the polarization or propagation direction of the light. Thus, it is not possible to achieve phase matching as in birefringent crystals. Clearly, for HHG a different approach to fulfil phase matching is required, i.e., which maximizes the generation of the $\mathrm{HH}$, by minimizing $\Delta k$. Looking into more detail, there are more essential differences between phase matching of SHG and HHG. These result from the much higher intensities required for HHG in gases $\left(10^{13}-10^{14} \mathrm{~W} / \mathrm{cm}^{2}\right)$ as compared to

\footnotetext{
${ }^{1}$ In some literature, wave-vector mismatch is sometimes defined as $\Delta k=k_{q \omega}-q k_{\omega}$, which leads to an opposite sign of each component and has no physical consequences.
} 
SHG $\left(\approx 10^{8} \mathrm{~W} / \mathrm{cm}^{2}\right)$. A first consequence is that strongly focused beams are to be used for HHG (to provide high intensities). As a result, the phase fronts of the drive laser beam acquire an additional geometrical phase in the focus compared to the standard plane wave description in SHG. A second consequence is that, due to the high intensities, the medium becomes ionized to an appropriate degree during the generation process, which leads to change of the dispersion, thereby rendering Eq. (2.49) incomplete (wrong). If a waveguiding geometry is used for providing high intensities over long interaction lengths [84], there are also contributions to the phase matching that stem from the waveguiding mechanism.

In this thesis, where waveguiding is not applied for HHG, the wave-vector balance can be written as follows, consisting of four terms (as indicated also in Fig. 2.7):

$$
\Delta k=\Delta k_{\text {atom }}+\Delta k_{\text {plasma }}+\Delta k_{\text {geo }}+\Delta k_{\text {dipole }} .
$$

The first contribution, $\Delta k_{\text {atom }}$, is due to the dispersion in the neutral atomic medium used, which is positive (pointing along the propagation direction) and can be expressed as

$$
\Delta k_{\text {atom }}=q(1-\eta) \frac{2 \pi P}{\lambda} \Delta n \text {. }
$$

Here, $\eta$ is the ionization fraction, which was described in Eq. (2.41), $P$ is the pressure of the gas in mbar, and $\Delta n$ is the difference between the indices of refraction of the gas per mbar at the drive laser wavelength, $\lambda$, and the $\mathrm{HH}$ wavelength, $\lambda / q$. The second contribution, $\Delta k_{\text {plasma }}$, originating from the free electrons in the plasma, on the contrary, is negative and becomes

$$
\Delta k_{\text {plasma }}=-\eta P N_{a} r_{e} \frac{q^{2}-1}{q} \lambda,
$$

where, $N_{a}$ is the number density of atoms per mbar, and $r_{e}$ is the classical electron radius. The third contribution, $\Delta k_{\text {geo }}$, resulting from the geometry of the wave propagation, e.g., the focusing of the drive laser beam near or inside the medium, is independent of the gas pressure. For a Gaussian drive laser beam, it equals the derivative of the Gouy phase and can be expressed as

$$
\Delta k_{\text {geo }}=-\frac{1}{z_{R}+\frac{\left(z-z_{0}\right)^{2}}{z_{R}}}(q-1),
$$

where, $z_{0}$ is the position of the focus and $z_{R}$ is the Rayleigh length. Because in a focus the distance between wave fronts is larger than in a plane wave. $\Delta k_{g e o}$ always takes negative values, with a minimum in the beam waist, where it is equal to $-(q-1) / z_{R}$. Since the Rayleigh length increases with the focal length, this contribution is only small for a loosely focused beam. The fourth contribution is due to the intrinsic phase of the radiating dipole formed upon recombination. The value of this phase depends on the trajectory and the momentum of the electron, which depend on the local intensity gradient along the focus as $\partial I / \partial z$ [85],

$$
\Delta k_{\text {dipole }}=-\alpha_{q} \frac{\partial I}{\partial z}=-\frac{2\left(z-z_{0}\right)}{z_{R}^{2}\left[1+\left(\frac{\left(z-z_{0}\right)}{z_{R}}\right)^{2}\right]^{2}} \alpha_{q} I_{0} .
$$


Here, $\alpha_{q}$ expresses the dependence on the electron trajectory and can be determined from the quantum mechanical theory described in Sec. 2.2.1. For instance, for the $21^{\text {st }}$ harmonic in argon, $\alpha_{q}=2 \times 10^{14} \mathrm{~cm}^{2} / \mathrm{W}$ for short trajectories and $\alpha_{q}=22 \times 10^{14} \mathrm{~cm}^{2} / \mathrm{W}$ for long trajectories [86]. It should be noticed that this wave-vector contribution can be made negative by focusing in front of the medium $\left(z<z_{0}\right)$, while placing the focus behind the medium $\left(z>z_{0}\right)$ yields a positive contribution.

Eq. (2.51) implies that phase matching $(\Delta k=0)$ can be achieved when the four described wave-vector contributions are balanced. When devising an according experiment, these four terms need to be properly adjusted via drive laser intensity, focusing geometry and the generating medium. The values for $\Delta k_{\text {atom }}$ and $\Delta k_{\text {plasma }}$ can be controlled by the gas pressure and the ionization fraction, which depend on the drive laser intensity. The values for $\Delta k_{g e o}$ and $\Delta k_{\text {dipole }}$ also vary with the position of the gas target (e.g., a gas cell or a gas jet) relative to the focal position of the drive laser beam, the Rayleigh length (i.e., the beam size and focusing optics), as well as the drive laser intensity.

In a tight focusing geometry, for which the Rayleigh length is on the order of tens of micrometers, phase matching is dominated by $\Delta k_{\text {geo }}$ and $\Delta k_{\text {dipole }}$. Another property of this geometry is that the generation volume, where the drive laser intensity reaches sufficiently high intensities on the order of $10^{14} \mathrm{~W} / \mathrm{cm}^{2}$, is very small. To still obtain an appreciable output, one can consider to increase the number of atoms in the interaction volume via the pressure (to a few bars). However, confining all the gas atoms to such a sufficiently small volume is especially challenging. To avoid the reabsorption of $\mathrm{HH}$ radiation caused by the gas atoms in the background, the maximum pressure is usually limited by the pumping capacity [87] of the vacuum system in which the gas is released into the laser beam, e.g., as a pulsed gas jet. In addition, due to the large number of gas atoms, the drive laser beam is strongly defocused by the ionized medium during the generation process, which results in a relatively low peak intensity of the drive laser and consequently lowers the overall $\mathrm{HH}$ conversion efficiency. On the contrary, in the loose focusing geometry, the Rayleigh length is in the order of tens of millimeters. The contribution from the intrinsic atomic dipole phase, $\Delta k_{\text {dipole }}$, is small and can usually be neglected. The phase matching can be achieved when the positive neutral dispersion $\left(\Delta k_{\text {atom }}\right)$ is compensated by the negative plasma dispersion $\left(\Delta k_{\text {plasma }}\right)$ and geometry dispersion ( $\Delta k_{\text {plasma }}$ ). With loose focusing, the interaction volume can be made much larger for maximizing the $\mathrm{HH}$ output. In this thesis, for maximizing the output and having a reduced criticality with regard to the focusing conditions, we have chosen to perform our experiments and calculations in the loose focusing geometry.

\section{QUASI-PHASE-MATCHING}

When inspecting the experimental conditions for which the phase-matching condition mentioned above can be fulfilled (Eq. (2.50), it can be concluded that fulfilling Eq. (2.50) allows only a very low ionization fraction. This low ionization fraction limits the drive laser intensity and thereby also the $\mathrm{HH}$ output that can be generated. At the same time, via Eq. (2.47), the shortest wavelength that can be generated (cut-off) becomes longer. Otherwise, i.e., when increasing the laser intensity in the hope for more HH output, due to the accumulated free electrons generated in the plasma, the plasma dispersion, $\Delta k_{\text {plasma }}$, becomes extremely large and overwhelms other dispersions and cannot be 
compensated by the other terms in Eq. (2.51). For example, by using a $40 \mathrm{fs}, 800 \mathrm{~nm}$ drive laser pulse to generate high-order harmonics in argon gas, only a less-than- $4 \%$ ionization fraction is allowed. This leads to a maximum harmonic order (the shortest wavelength) that can be phase-matched of about the $35^{\text {th }}(\approx 24 \mathrm{~nm})$ [88]. Simply in-

no phase-matching

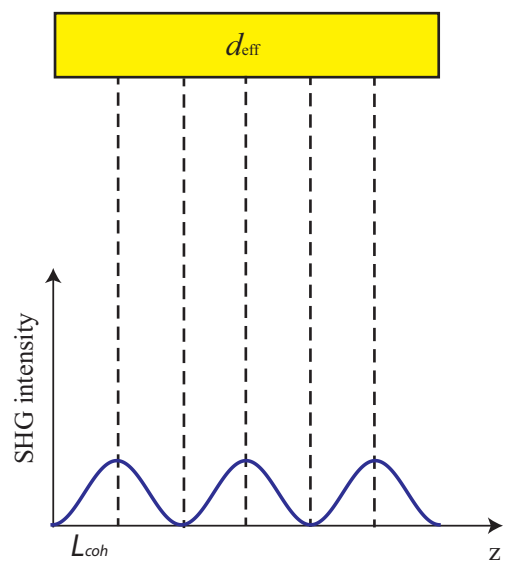

quasi phase-matching (periodically poled crystal)

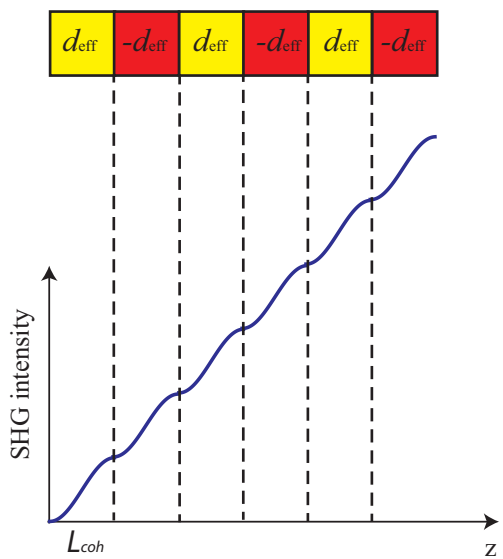

Figure 2.8: Schematic of SHG in the absence of phase matching (left) and when a quasiphase matching (right) is achieved. In a homogeneous nonlinear crystal, the generated second harmonic field is out of phase respect to the generated field at the beginning of the crystal after one coherence length. In periodically poled crystals, the crystal orientation is switched after one coherence length such that the effective nonlinear coefficient changes sign and the new generated second harmonic field in phase again with the previous generated field and interfere constructively.

creasing the drive laser intensity to further increase the generated order, thereby accepting a situation that is not phase matched, will result in an oscillating harmonic yield along the propagation direction, as is depicted in Fig. 2.6 (right). The regions where the harmonic yield increases (decreases) are called in-phase (out-of-phase) regions. In order to improve the conversion efficiency for these higher order harmonics, a solution can be so-called quasi-phase-matching (QPM), a technique that is well-known and often applied for SHG in periodically-poled nonlinear crystals. To illustrate the working principle of QPM, Fig. 2.8 summarizes the situation for SHG. At the beginning of the crystal, the generated second harmonic field grows along the propagation direction, $z$. After one coherence length, the generated second harmonic field is due to dispersion out of phase by $\pi$ with respect to the second harmonic field generated at the beginning of the crystal, resulting in the onset of back-conversion with the second harmonic intensity decreasing again vs. $z$. The idea of QPM in crystals where the sign of the nonlinear coefficient $\left(d_{\mathrm{eff}}\right.$ in Eq. (2.49)) can be reversed by reversing the orientation of the crystal structure, is to correct the phase relation by switching the crystal orientation after one coherence length. In this case, the second harmonic field generated in the reversed regions will interfere constructively with the field generated at the beginning of the crystal and contribute to a steady build-up also after the first coherence length. A periodic repetition of the crystal 
orientation can be obtained in certain crystals with high voltage induced re-orientation (periodic polling [89]), leading to a steady monotonic growth of the second harmonic intensity along the propagation direction.

Following this basic concept that was actually proposed even before the invention of birefringent phase matching, there have been a number of realizations of QPM also for HHG, though mainly in a waveguiding geometry [90]. However, a central problem
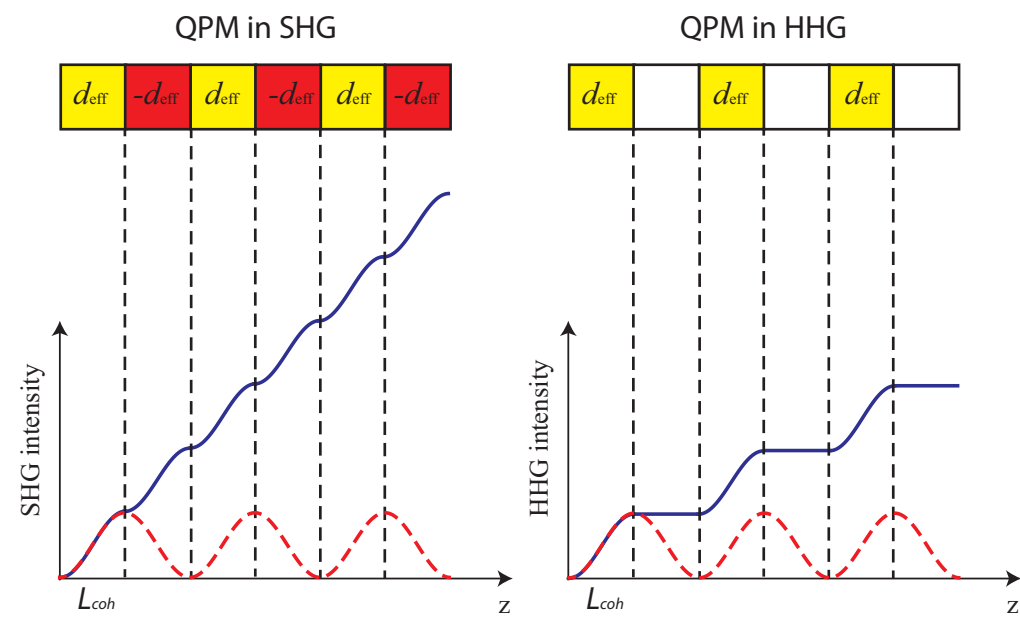

Figure 2.9: Comparison of quasi-phase matching in SHG (left) and HHG (right). The QPM for SHG is achieved by periodically reversing sign of the nonlinear coefficient in crystals, while the QPM for HHG, is achieved by suspending (suppressing) the generation process in the out-of-phase region. The length of each region corresponds to the coherence length $L_{c o h}$, where the generated harmonic field experiences a phase shift of $\pi$. The intensity yields along the propagation axis, z, for both SHG and HHG in the absence of phase matching (red dashed curves) are also plotted, respectively.

in applying a QPM scheme for HHG is, that the media suitable for HHG are usually gaseous. This means that other than crystals, the HHG media are generally isotropic and it is extremely difficult or impossible to re-orient all gas particles into a desired direction. Rather, the idea of QPM for HHG is, instead of introducing some periodical modulation of the orientation of radiating dipoles, introducing a periodic modulation of the local strength of the generation process throughout the medium, as is illustrated in Fig. 2.9. Instead of reversing the nonlinear susceptibility of the gas after one coherence length, one just suspends (suppresses) the HH interaction to prevent back-conversion until another coherence length is reached, where the involved waves are in phase again for further growth of the high-order harmonics. This process can be repeated periodically as is depicted in Fig. 2.9 and should yield a steady monotonic growth of the $\mathrm{HH}$ output vs. interaction length. In the spatial Fourier domain such additional periodic modulation is equivalent to introducing an extra wave vector, $K_{Q P M}$, that compensates the wave-vector mismatch originating from the plasma dispersion, which would otherwise dominate in highly ionized media. The total wave-vector mismatch, $\Delta k^{\prime}$, for HHG 
including QPM can be expressed as

$$
\Delta k^{\prime}=\Delta k-K_{Q P M}
$$

where $\Delta k$ is the wave-vector mismatch in a homogeneous medium given by Eq. (2.51).

\section{REABSORPTION}

The conversion efficiency of HHG is not only dependent on the phase mismatch, as mentioned above, but also on the absorption by the generating medium. Once phase matching, $\Delta k=0$, is achieved, or more practically once $\Delta k$ is minimized such that the coherence length, $L_{c o h}$, is set to values longer than the given length of the medium, $L_{m e d}$, the harmonic yield is expected to grow proportional to the square of $L_{\text {med }}$ in the plane wave approximation. However, due to its short wavelength and photon energies comparable or larger than atomic ionization energies, the generated harmonic radiation covering the range from XUV to the soft X-rays is strongly absorbed by most media including the generating gas, even if a noble gas was chosen to provide increased ionization energies. In practice, the effective length of the generating medium is simply limited by the reabsorption. According to the work published by Constant et al. [91], assuming a homogeneous gaseous medium and a constant single-atom response over the whole generation volume, the on-axis harmonic yield is proportional to

$$
I_{H H G} \propto \frac{4 L_{a b s}^{2}}{1+4 \pi^{2}\left[L_{a b s}^{2} / L_{c o h}^{2}\right]} \times\left[1+\exp \left(-\frac{L_{m e d}}{L_{a b s}}\right)-2 \cos \left(\pi \frac{L_{m e d}}{L_{c o h}}\right) \exp \left(-\frac{L_{m e d}}{2 L_{a b s}}\right)\right],
$$

where $L_{a b s}$ is defined as the length where the harmonic yield drops to $1 / e$ of the initial value [92]. One conclusion is that, as is illustrated in Fig. 2.10, in order to ensure that the phase-matched harmonic yield is only limited by the reabsorption and reaches at least half of the maximum value, the conditions (green area in Fig. 2.10)

$$
L_{m e d}>3 L_{a b s} \text { and } L_{c o h}>5 L_{a b s}
$$

should be fulfilled. In the case when phase matching is not available and QPM is required for generating sufficient higher-order harmonics at shorter wavelengths, the reabsorption of these wavelengths in the generating medium is usually much weaker. Fig. 2.11 shows an example of the calculated absorption length, $L_{a b s}$, between $10 \mathrm{~nm}$ and 60 $\mathrm{nm}$ in argon gas at a pressure of $30 \mathrm{mbar}$ [92]. It can be clearly seen that on the longer wavelength side ( $30 \mathrm{~nm}-60 \mathrm{~nm}$ ), where phase matching can be successfully achieved, the absorption length is rather short, specifically above $35 \mathrm{~nm}$ (HH23)), less than $1 \mathrm{~mm}$, while on the short wavelength side (10 nm - $30 \mathrm{~nm})$, the absorption length grows dramatically and reaches its maximum around $25 \mathrm{~nm}$. Considering a typical experimental condition of HHG in argon gas with a medium length of $5 \mathrm{~mm}$, the generation of harmonic orders above $35^{\text {th }}(\approx 24 \mathrm{~nm})$ cannot be phase matched. For efficient generation of these higher orders implementation of QPM is required. Since the absorption length at these orders is longer than the medium length, the reabsorption in the generating medium usually can be neglected. 


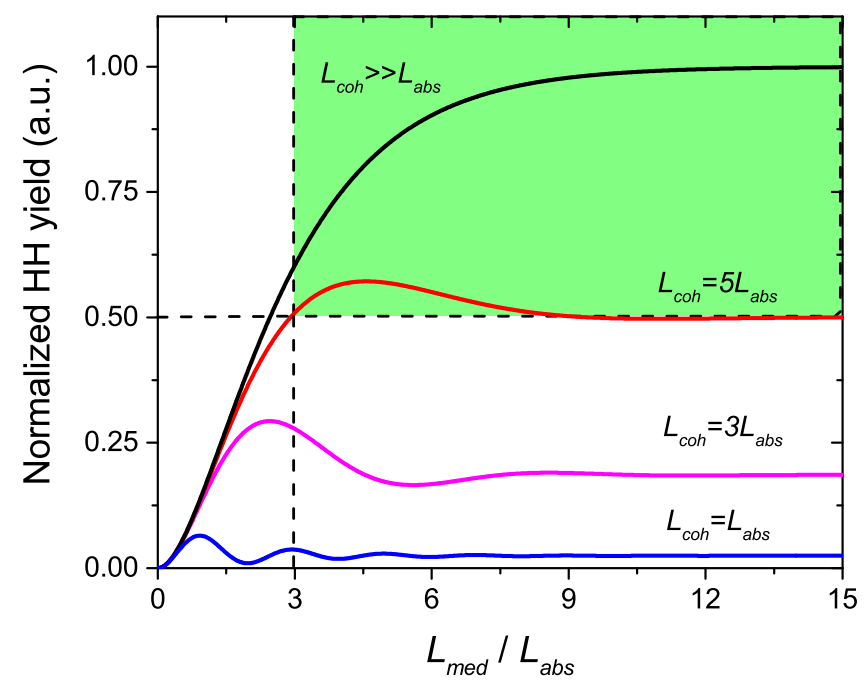

Figure 2.10: Normalized harmonic yield as a function of medium length in terms of absorption length, $L_{a b s}$, for four different coherence lengths, $L_{c o h}$. The green area indicates the criteria $L_{m e d}>3 L_{a b s}$ and $L_{c o h}>5 L_{a b s}$ are fulfilled.

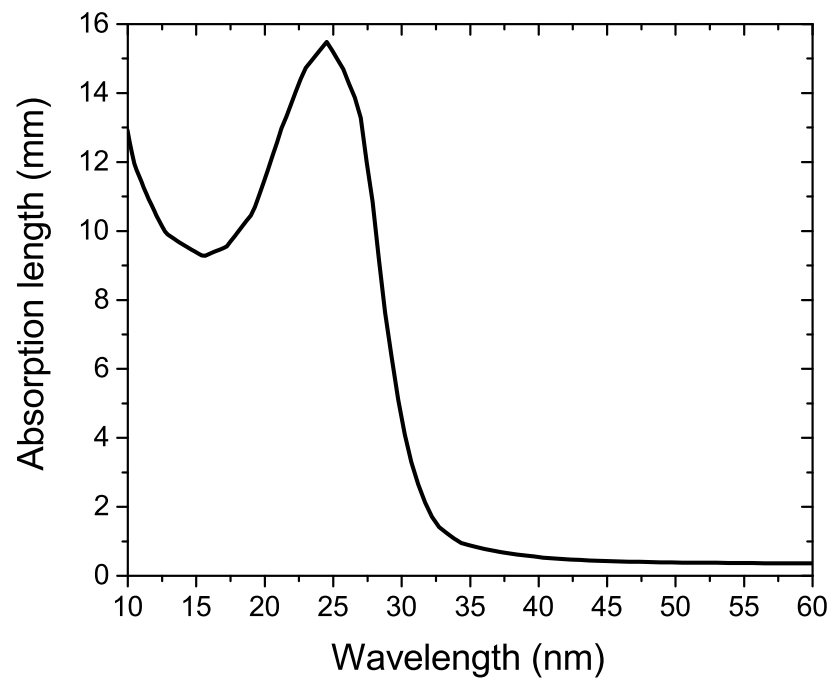

Figure 2.11: Calculated absorption length in argon gas at a pressure of 30 mbar from 10 $\mathrm{nm}$ to $60 \mathrm{~nm}$ based on data from [92]. 


\section{TRANSIENT CHARACTER OF PHASE MATCHING AND QUASI-PHASE MATCHING}

Apart from the above discussion about realizing phase matching or quasi-phase-matching in HHG, there is an absolutely central issue that has not been considered so far and which is often neglected in spite of its importance. Namely, the phase-matching conditions, particularly at the intensities required for HHG, are highly transient due to the time-dependent growth of the ionization fraction during each drive laser pulse. As a result, the values of $\Delta k_{\text {atom }}$ and $\Delta k_{\text {plasma }}$ vary strongly and on an ultrafast time scale. It should be noted that the ionization of the medium is not a troublesome, perturbing effect that needs to be avoided or removed. Ionization is, namely, the very operating mechanism underlying HHG. In other words, ionization is required to obtain a HH response of the atoms (tunnel ionization). This situation is comparable with, e.g., four-wave mixing with ultrashort pulses in standard optical materials. There, the main interaction is enabled by the third-order nonlinearity, while, the same nonlinearity gives rise to the Kerr effect that manifests as an intensity and thus time-dependent refractive index. The latter transforms phase matching into a transient process, just as is the case here with HHG. Strictly speaking, the phase-matching condition cannot be fulfilled for the entire laser pulse. This opens the question whether for phase matching an optimum moment in time, within the drive laser pulse, needs to be chosen. The harmonic output generated with phase matching at the peak intensity of the drive laser pulse does not necessarily correspond to the time-integrated maximized harmonic yield.

Furthermore, such a highly transient character of phase matching has also a direct influence on determining the modulation period for achieving QPM in HHG and thereby obtaining a time-integrated maximized HH output. Various schemes to demonstrate or make use of QPM in HHG have been proposed and developed already, although these seem to be much less successful in comparison with low-order conversion in standard nonlinear crystals. For instance, the use of a small set of periodically spaced dual-gas jets has shown to increase the conversion efficiency for the harmonic orders up to the $41^{\text {st }}$ order $(\approx 19 \mathrm{~nm})$ in argon $[45,93,94]$. A train of tunable counter-propagating pulses in a hollow-core waveguide has been used to introduce a spatially periodic ionization pattern for generating high-order harmonics of photon energies of several hundred eV [41]. Other schemes include waveguides with a spatially periodic diameter [39], multimode beating in a capillary [40] and polarization-beating in a birefringent waveguide [95, 96]. In all these QPM schemes, people claimed that HHG is confined to the in-phase region and suppressed in the out-of-phase regions, as described in Fig. 2.9. However, all of them have silently ignored the transient character of phase matching in HHG, and thereby also of QPM in HHG. Instead of carefully analyzing the time-dependence of phase matching, they usually select a fixed periodic structure for the highest harmonic output based on an unproven assumption, namely, that the quasi-phase matching period has to be chosen as equal to twice the coherence length calculated at the peak intensity of the drive laser pulse. This is based on the argument that then the nonlinearly induced dipole moment of the individual atoms is the highest. They hope that this will also result in the highest harmonic output for the entire pulse without any detailed calculations.

In this thesis, we propose the first comprehensive time-dependent QPM model for HHG that takes into account all the transient effects in phase matching for the important case of quasi-phase matching, and we determine the appropriate modulation period 
for maximizing the global (time-integrated) harmonic yield. A detailed description and discussion of the model will be presented in Chapter 6 . 



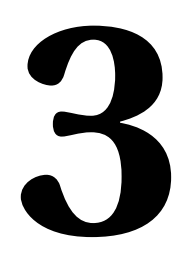

\section{EXPERIMENTAL PREPARATION}

This chapter is addressed to the experimental apparatus constructed in the lab. The apparatus is applied for two main experiments described in this thesis. The first experiment is the optical characterization of cluster formation in a supersonic argon gas jet. The second experiment is the high-order harmonic generation in such a supersonic jet. The purpose of this chapter is to provide detailed information for a better understanding of the experimental results presented in Chapter 4 and Chapter 5. 


\subsection{Cluster Jet CHARACTERIZATION SETUP}

Within the work described in this thesis, there is a great need for an easy and reliable method for characterizing cluster jets, such as with respect to the average cluster size, $\langle N\rangle$, density, $n_{c}$, and total atomic number density, $n_{a}$. We have generated an argon cluster jet using a supersonic slit nozzle, which is also called planar nozzle. We begin the description of the supersonic nozzle in Section 3.1.1. Next, in Section 3.1.2 we introduce the setup that we constructed for performing interferometry and quantitative measurements of Rayleigh scattering, which was used to obtain experimental information about measured values for the cluster size and density.

\subsubsection{SUPERSONIC NOZZLE}

The supersonic nozzle used in this thesis has been designed for having a rectangular shaped exit with targeted dimensions of $1.0 \times 5.0 \mathrm{~mm}^{2}$ as well as a rectangular shaped throat with diemensions of $0.3 \times 1.0 \mathrm{~mm}^{2}$. The expansion half-angle of the nozzle is set to $14^{\circ}$. A detailed technical drawing of the nozzle can be found in Appendix A [64]. Manufacturing such a nozzle with the intended dimensions is of great challenge, especially for achieving the required size and narrow (sub-millimeter) shape for the throat of the nozzle. To obtain a high accuracy in the order of tens of micrometers for the specified shape of the nozzle, fabrication was performed by electrical discharge machining. Fig. 3.1 shows an optical microscopic image of the real shape of the throat of the nozzle. It is

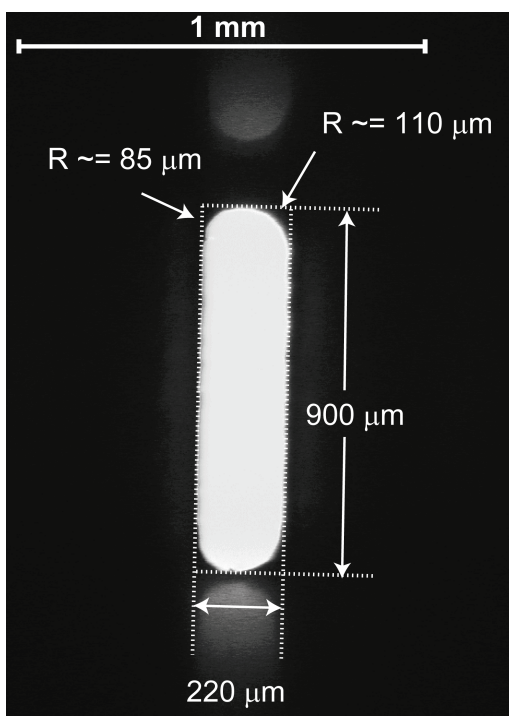

Figure 3.1: Image of the throat of the nozzle captured under an optical microscopy. The throat has a shape slightly deviating from a rectangular shape and is about $900 \mu \mathrm{m}$ long and $220 \mu \mathrm{m}$ wide.

clearly seen that, instead of an ideal rectangular shape, the throat is only approximately rectangular due to the rounded shape of the corners. Furthermore, the real throat area amounts to about $0.22 \times 0.90 \mathrm{~mm}^{2}$, which is slightly smaller than the designed area. This 
real measured area of the throat is what we used as input value for the flow calculation, and for the calculation of cluster formation as discussed in Chapter 4.

Due to technical limitations on the capacity of the vacuum pumps and to avoid excessive gas consumption as typical for a continuous gas or cluster jet, we generate the argon clusters with a pulsed gas flow. For this purpose, the supersonic nozzle is mounted on top of a pulsed valve operated magnetically with a solenoid (Parker, 9 series), as is shown in Fig. 3.2. The current for valve activation is generated with a home-made elec-

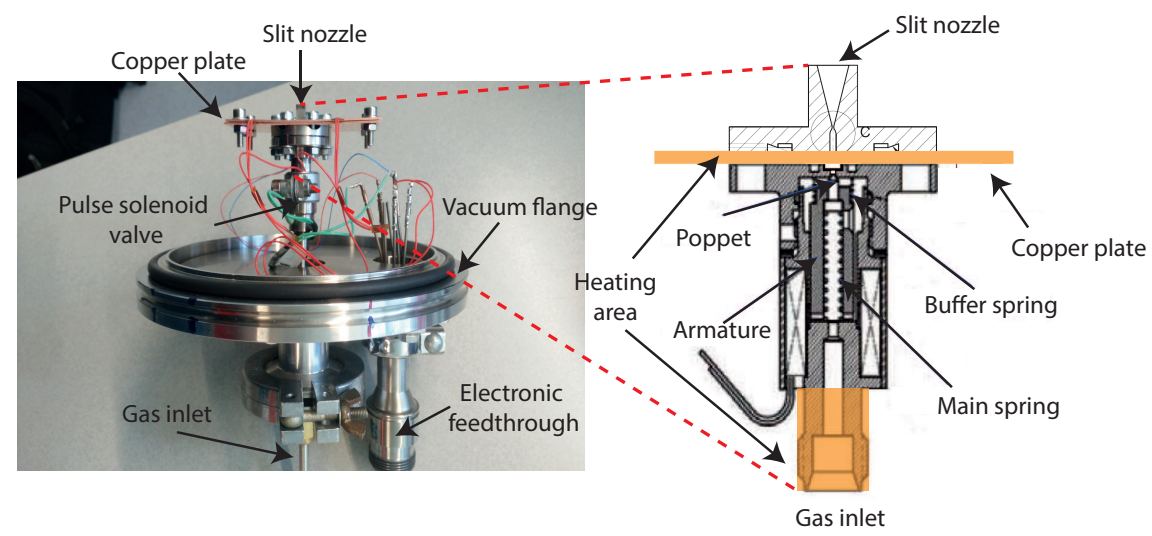

Figure 3.2: Real image (left) and enlarged schematic (right) of the slit nozzle mounted on the top of the solenoid valve. The whole assembly is attached to the vacuum flange with both gas and electronic feedthrough.

tronic driver developed by T. A. W. Wolterink in the LPNO group. The driver works as follows: when the valve is in operation, the magnetic field produced by a high voltage $(\approx 300 \mathrm{~V})$ and thus driven by a high current quickly draws the armature and the attached poppet downwards the solenoid, thus quickly opening the valve. After 180 microseconds, the voltage is reduced to $12 \mathrm{~V}$ to avoid damage by excessive currents, however the voltage is sufficient to hold the armature and keeps the valve open. When the voltage is withdrawn after an adjustable time, the main spring pushes the poppet back in place which seals the orifice. A buffer spring located between the armature and the orifice prevents damage that may otherwise be caused by too fast a collision between the poppet and the orifice. The whole assembly of the nozzle and the valve is connected to a vacuum flange with both gas and electronic feedthroughs. The stagnation pressure, $p_{0}$, applied to the valve is controlled by two commercial gas regulators and can be varied up to 70 bar. An accuracy of 0.5 bar in the high-pressure range between 5 bar and 70 bar is achieved, while a higher accuracy of $200 \mathrm{mbar}$ is available in the low-pressure range between 0 bar and 5 bar. According to the specification, such a pulsed solenoid valve allows a maximum repetition rate of $100 \mathrm{~Hz}$. However, due to the pump capacity in our setup, the rate is normally lowered to $1 \mathrm{~Hz}$ or below during the experiments. Moreover, the temperature of the gas, $T_{0}$, in the valve can also be increased, which is essential to influence the density and the size of the generated clusters for the HHG experiments performed in Chapter 5. This is achieved by heating the whole nozzle as well as the valve. For heating, we have installed two Polyimide Thermofoil heaters (Minco) in between two 
copper plates which are tightly fixed on the top of the valve (see. Fig. 3.2). Due to the high heat conductivity of copper, the whole assembly can be easily heated uniformly. In addition, we also heat the bottom part of the valve to avoid a temperature gradient inside the valve. The heating power and temperature is controlled by a PID temperature controller (Thorlabs, TC200-EC). During the experiment, the temperature of the valve can be set to any temperature between room temperature and $105^{\circ} \mathrm{C}$ with an accuracy of $0.2^{\circ} \mathrm{C}$.

\subsubsection{INTERFEROMETRY AND RAYLEIGH SCATTERING SETUP}

To characterize the spatial distribution of gas and clusters throughout the supersonic argon jet, we have built an experimental setup, as is shown schematically in Fig. 3.3. The setup includes a Mach-Zehnder interferometer and a detection of single-pass Rayleigh scattered light. The jet is located in the upper arm of the interferometer. The slit nozzle

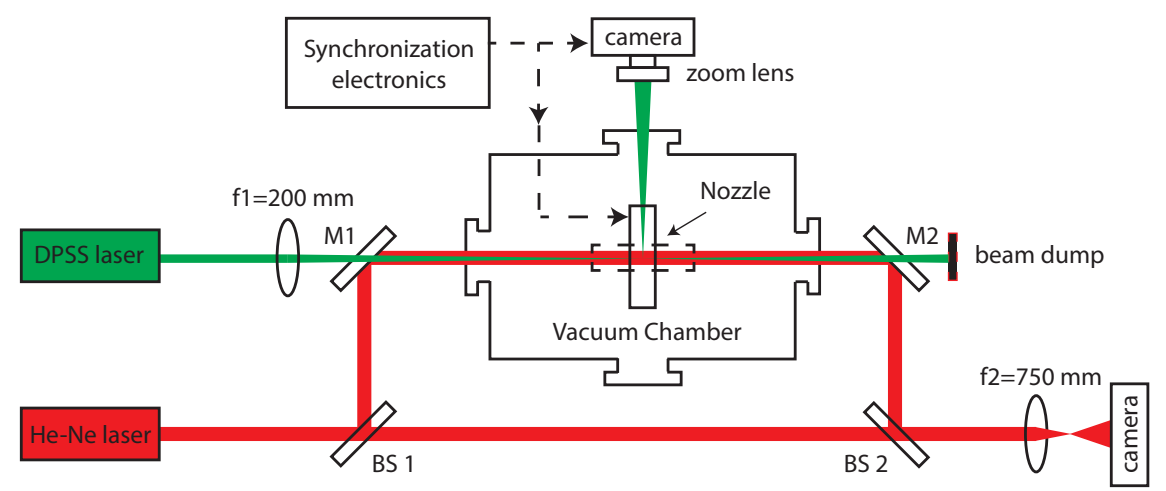

Figure 3.3: Schematic of the experimental setup; Red beam path: Mach-Zehnder Interferometer; Green beam path: beam paths for imaging of Rayleigh scattering. The nozzle can be rotated by 90 degree (dashed) for a transverse interferometric measurement. Dashed arrows represent the electronic timing synchronization path.

can be rotated about its central symmetry axis, to orient it either perpendicular or parallel to the laser beam, i.e., to propagate the laser beams through the short dimension (width) or the long dimension (length) of the jet. To measure the total atomic number density distribution in the cluster jet, we inject the beam from a linearly polarized $\mathrm{He}-\mathrm{Ne}$ laser (JDS Uniphase, wavelength $633 \mathrm{~nm}$ ) via a $50 \%$ beam splitter (BS1) into the interferometer. After superposition with light beam from the reference arm behind beam splitter (BS2), interference fringes containing information about the density distribution of the jet are imaged onto a CMOS camera (PixeLink, PL-A741) using a lens with $750 \mathrm{~mm}$ focal length.

To quantify the number density and size of clusters via Rayleigh scattering, the beam of a linearly polarized continuous-wave diode pumped solid state laser (DPSSL, Shanghai Laser Optics Century, operating at $532 \mathrm{~nm}$ at an output power of $100 \mathrm{~mW}$ ) was focused at a distance of $1 \mathrm{~mm}$ above the nozzle exit using a lens with a focal length of 20 $\mathrm{cm}$. The nozzle is positioned to be parallel to the laser beam (beam traverses the jet 
along its long dimension). The Rayleigh length of the beam is around $1 \mathrm{~cm}$. Scattered light from the jet is recorded under an angle of 90 degree with another camera (PixeLink, PL-A741) having a lens with a focal length of $50 \mathrm{~mm}$. For the absolute calibration of the Rayleigh scattering signal, scattering from several known densities of gas atoms (argon) in the field of view imaged by the camera was recorded. For this purpose, the entire vacuum chamber was filled with argon gas at different static pressures between 300 mbar and 900 mbar, while keeping the other setup parameters unchanged, specifically, the collection angle, the optical system transmission as well as the gain of the camera. Fig. 3.4 shows a typical image of Rayleigh scattering from the argon gas filled inside the entire chamber at a static pressure of 700 mbar. Both cameras and the driver for the gas valve in the experiment are electronically synchronized (Thales, Intelligence Synchronization Electronics for Optics).

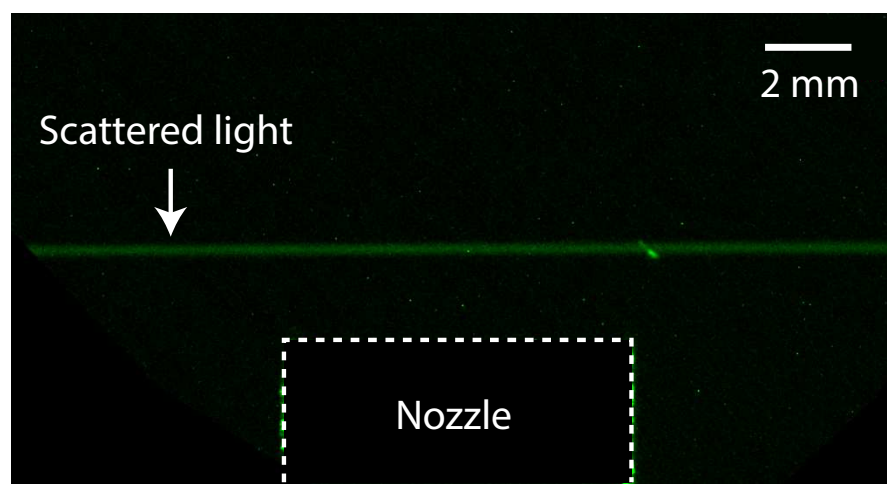

Figure 3.4: A typical Rayleigh scattering image of argon gas filled inside entire chamber at a static pressure of 700 mbar.

In the experiments on cluster formation, the argon gas is supplied to the backside of the valve (and nozzle) with varying stagnation pressures of up to 70 bar. The length of the opening time interval of the valve is set to a sufficiently long value (5 ms) to reach a steady-state flow. Steady-state flow is confirmed by monitoring the scattering signals as a function of time with a photomultiplier at the position of the camera, as is shown in Fig. 3.5. During repetitive valve operation, the time averaged background pressure lies in a range between $10^{-3}$ and $10^{-2}$ mbar depending on the stagnation pressure applied. In order to attain a good signal-to-noise ratio and to reduce the effect of shot-to-shot deviations in the gas flow, all the measurements are averaged over 20 gas shots.

\subsection{HIGH-ORDER HARMONIC GENERATION SETUP}

To drive HHG in the supersonic argon jet described in the above section and diagnose the generated harmonic beam such as the beam profile and the spectrum, a HHG beamline has been designed and constructed during this thesis. Fig. 3.6 shows a schematic of the full setup and a few photographs of this setup are shown in Appendix B. The solid arrows depict the optical paths, while the dashed arrows indicate the electronic timing synchronization paths. The entire setup can be coarsely divided into three parts, namely 


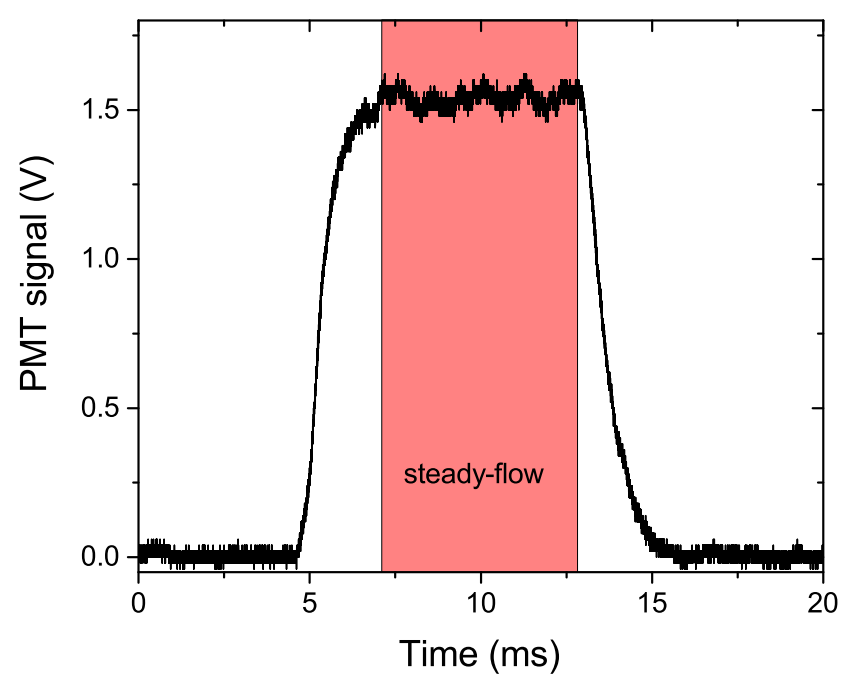

Figure 3.5: Measured Rayleigh scattering signal from the argon cluster jet at a stagnation pressure of 20 bar by a photomultiplier. A time interval (longer than $5 \mathrm{~ms}$ ) when a steadyflow is reached is indicated by the red filled area.

the high-power laser system (described in Section 3.2.1), the HHG target chamber (see Section 3.2.2) and the harmonic diagnostic system (see Section 3.2.3).

\subsubsection{HigH POWER FEMTOSECOND LASER SYSTEM}

The high-power femtosecond laser system used for HHG is a modified chirped pulse oscillator amplifier system (Coherent. Inc). It consists of a Ti:Sapphire (Ti:Sa) oscillator (Mantis) and a two-stage amplifier (Legend Elite Duo-USP-HP) including a regenerative amplifier and a single pass amplifier. The laser has a repetition rate of $1 \mathrm{kHz}$ and a nominal maximum pulse energy of $7 \mathrm{~mJ}$ at a central wavelength of $798 \mathrm{~nm}$. The pulse duration of the laser is about $40 \mathrm{fs}$ (see Fig. 3.7), as measured by an optical autocorrelator (APE PulseCheck). Together with the bandwidth $(\approx 27 \mathrm{~nm}$, see Fig. 3.7) measured by a spectrometer (OceanOptics USB2000), a time bandwidth product of 0.5 is obtained, which indicates that the pulses are almost Fourier-limited. Furthermore, an acousto-optic programmable dispersive filter (AOPDF, Dazzler HR-800/T1, fastlite ${ }^{1}$ ) [97] is installed between the oscillator and amplifier. This component was originally used for our previous experiemnts on spectral control of HHG experiments in waveguide capillaries [34]. In this thesis, the Dazzler is used to compensate the extra dispersion of the transmissive optical components including the half-wave plates, the focusing lens and the entrance window $\left(\mathrm{W} 1, \mathrm{MgF}_{2}\right)$ for transmitting the beam into the vacuum chamber. For driving high-order harmonics and avoiding any major self-phase modulation along

\footnotetext{
${ }_{1}$ http://www.fastlite.com/en/ar824665-823529-Dazzler.html
} 


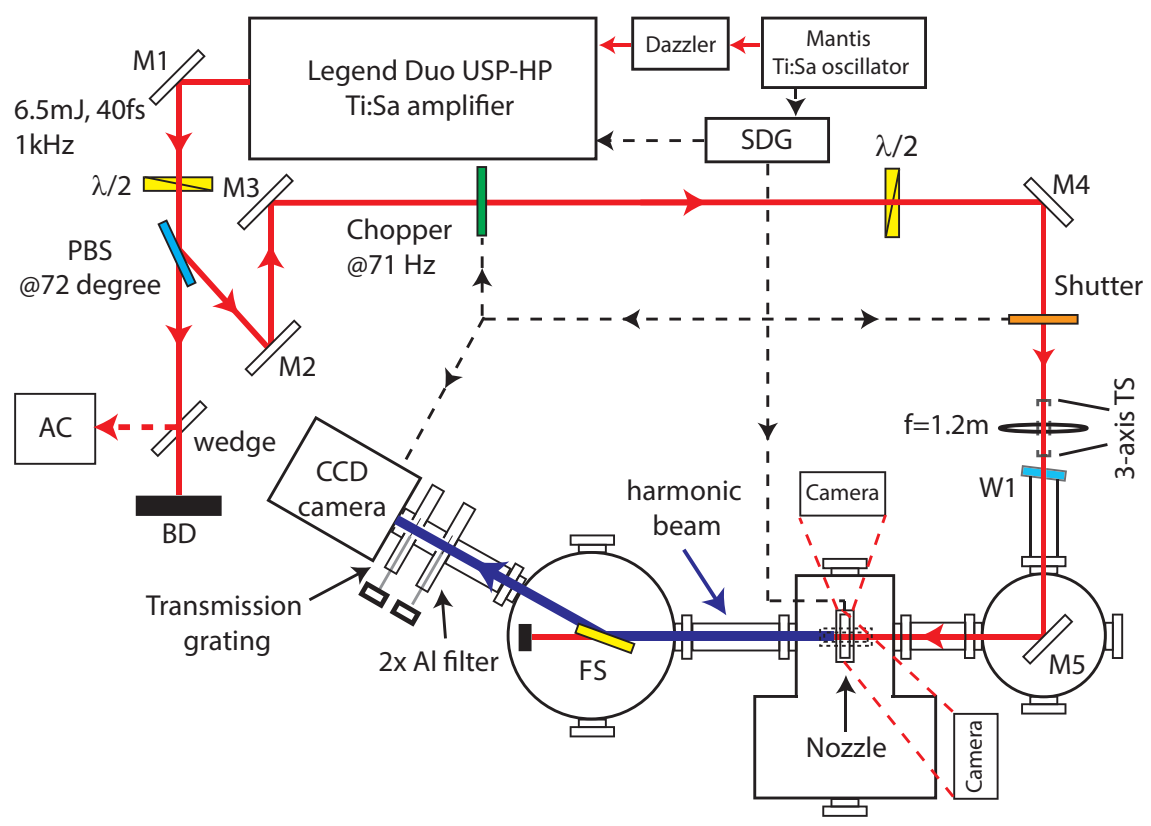

Figure 3.6: Schematic of the full HHG setup. The high-order harmonics are driven by focusing a Ti:Sa laser beam consisting of high-energy ultrashort pulses into a supersonic argon jet produced by a nozzle. The generated harmonic beam is separated from the drive laser beam first by a uncoated fused silica plate and additionally by two thin aluminium (Al) filters. The harmonic beam profile and spectrum are analyzed by the XUV spectrometer including a home-built transmission grating and a back-illuminated XUV CCD camera. The timing synchronization is controlled with a set of synchronization and delay generators. Combing with a mechanical chopper and a mechanical shutter, single-shot measurement can be realized. AC: auto-correlator, BD: beam dump, FS: fused silica plate, PBS: polarizing beam splitter, SDG: synchronization and delay generator and TS: translational stage.
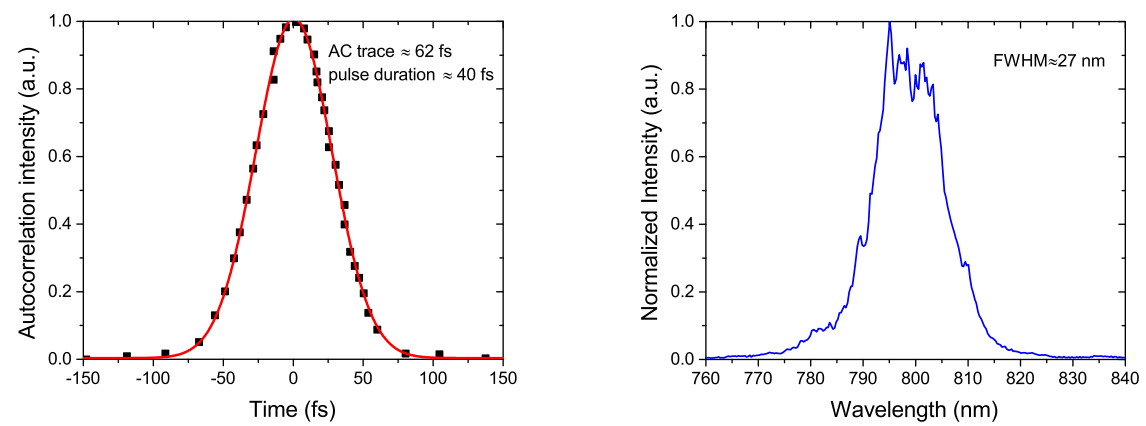

Figure 3.7: Measured autocorrelation trace (left) and spectrum (right) of the laser pulse. The real pulse duration is derived to be around $40 \mathrm{fs}$ by assuming a Gaussian shape and the bandwidth of the laser, $\approx 27 \mathrm{~nm}$, is determined at the full-width of the spectrum a $1 / e^{2}$ of its maximum. 
the path into the vacuum chamber, only a fraction of the laser pulse energy is used, typically 1 to $3 \mathrm{~mJ}$. The used fraction was adjusted by a variable attenuator consisting of a rotatable half-wave plate followed by a polarizing beam splitter (CVI, low dispersion, working at $72^{\circ}$ angle of incidence), as is shown in Fig. 3.6. Such arrangements ensure that the shortest pulse duration is obtained after the laser beam is sent into the vacuum chamber. The laser beam displayed a slightly ellipticity (1:2 aspect ratio) and a slightly off-unity beam parameter $\left(M_{x}^{2}=1.1 ; M_{y}^{2}=1.3\right)$ [98]. These values are taken into consideration for the calculations as well as for performing the experiments as descried in Chapter 5 and Chapter 6. The laser beam described so far is sent into the HHG target chamber and cluster jet to generate high-order harmonics.

\subsubsection{HHG TARGET CHAMBER}

To enable the HHG, the supersonic nozzle with the solenoid pulsed valve described in Section 3.1.1 is directly mounted and fixed inside the vacuum chamber. As is shown in Fig. 3.6, the laser beam is focused with a plano-convex lens of $1200 \mathrm{~mm}$ focal length into the vacuum chamber achieving a so-called loose focusing geometry, where the Rayleigh range is much longer than the interaction length. The measured focal spot has an elliptical transverse profile (close to a Gaussian) with a waist of $100 \times 75 \mu \mathrm{m}$. The focusing lens is mounted on a three-axis $(\mathrm{x}, \mathrm{y}, \mathrm{z})$ translational stage such that, together with the mirror (M4), the focal position of the beam can be accurately controlled longitudinally and transversely with respect to the position of the nozzle. The nozzle is fixed in two alternative manners, either oriented along or perpendicular to the laser propagation axis. The laser beam is usually fixed about $1 \mathrm{~mm}$ above the nozzle and its focal position can be adjusted from about $5 \mathrm{~mm}$ in front of to $5 \mathrm{~mm}$ behind the center of the nozzle with an accuracy of $100 \mu \mathrm{m}$. To avoid absorption of the generated harmonics in the

side view

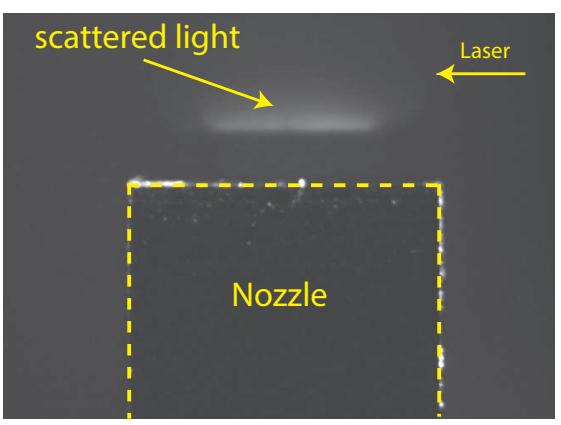

top view

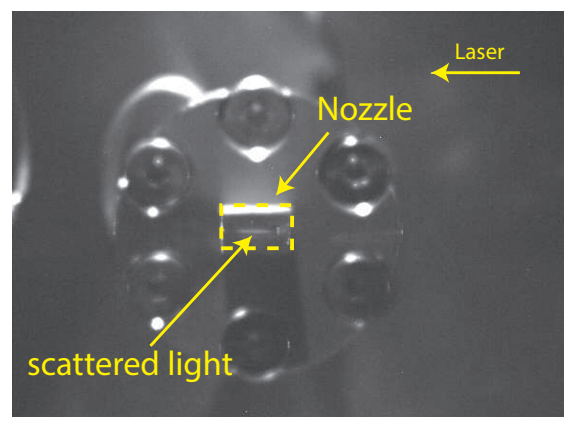

Figure 3.8: Side (left) and top (right) view of the scattered light from the argon jet irradiated by the Ti:Sa laser pulse. The laser beam is coming from the right side of the image. The stagnation pressure on the gas valve is set to 20 bar. Note: these two images were captured before the heating assembly for the nozzle is installed.

background gas which is usually unavoidable with a finite pump capacity, the solenoid pulsed valve is operated at a repetition rate of $1 \mathrm{~Hz}$, as mentioned before. The pressure in the vacuum chamber is normally kept in the range of $10^{-6}$ mbar by a $750 \mathrm{~L} / \mathrm{s}$ turbo 
pump, while during the operation of the valve, the time averaged background pressure rises up to $2 \times 10^{-3} \mathrm{mbar}$. The repetition rate of the laser is reduced as well, to $71 \mathrm{~Hz}$ in a first step, by inserting a mechanical chopper (MC20008B-EC, Thorlabs Inc) into the beam path to prevent undesired HHG from the remaining background gas which is not pumped away promptly as well as the optical damage in the detection system described in Section 3.2.3. Combining with a mechanical optical shutter (SH05, Thorlabs Inc), the repetition rate of the laser can be further reduced down to $1 \mathrm{~Hz}$, which allows single-shot detection of the harmonic beam profile. Two cameras (Pixelink, PL-A741) are installed behind two view ports (top and side view) of the vacuum chamber to monitor scattered light from the laser-cluster interaction. This provides a visual inspection on the laser beam alignment with respect to the nozzle. Fig. 3.8 shows two typical captured images of the scattered light from the supersonic argon jet irradiated by the Ti:Sa drive laser with $3 \mathrm{~mJ}$ pulse energy. For these two images, the stagnation pressure on the gas valve was set to 20 bar. All electric synchronization among the laser pulses, gas valve, chopper, shutter and cameras is achieved by a Synchronization and Delay generator (SDG, Coherent Inc) and several digital delay generators (DG535, Stanford Inc). The jitter between the laser pulses and the opening of the gas valve can be controlled with an accuracy better than $50 \mu \mathrm{s}$, which is mainly induced by the instability of the mechanical chopper.

\subsubsection{HARMONIC DIAGNOSTIC SYSTEM}

After the high-order harmonics are generated in the argon jet, the generated harmonic beam co-propagates with the drive laser beam towards a harmonic diagnostic system. To prevent any damage of the detection system by the drive laser beam, in particular, to protect the XUV CCD camera, the drive laser beam and the harmonic beam are separated with a large contrast in two steps. First, an uncoated fused silica plate is placed half a meter behind the nozzle in the exiting beams. The plate is placed at $75^{\circ}$ angle of incidence (relative to the normal), which is close to the Brewster angle for the drive laser beam. For p-polarized light, the reflectance for the drive laser beam is measured to be about $10 \%$, while for the generated harmonic beam between $17 \mathrm{~nm}$ to $80 \mathrm{~nm}$, it is calculated to be above $20 \%$ [92, 99, 100] (see Fig. 3.9). For achieving the named suppression factor for the drive laser, the polarization of the drive laser beam is carefully controlled by inserting another tunable half-wave plate in front of the focusing lens. Next, two $200 \mathrm{~nm}$ thick Al filters placed in series are used to fully block the drive laser radiation via reflection. The filters act as a band pass for the harmonic radiation, transmitting more than $40 \%$ of the harmonic radiation in the wavelength range of $17 \mathrm{~nm}$ to $80 \mathrm{~nm}$ [92], as is shown in Fig. 3.9. Behind the filters, the transmitted harmonic beam is sent into a home-built XUV spectrometer that consists of a transmission grating and a back illuminated XUV CCD camera (PIXIS-XO 2048B, Princeton Instruments). The camera is normally cooled down to $-60^{\circ} \mathrm{C}$ to reduce the noise during the operation. The spectrometer requires high vacuum for its operation, which was provided with an extra $90 \mathrm{~L} / \mathrm{s}$ turbo pump directly connected to the spectrometer. This approach kept the background pressure below $10^{-5}$ mbar during all measurements. The harmonic beam profile is detected by the same XUV camera after shifting the diffraction grating out of the beam path. Principally, the camera can be regarded as being located in the far-field of the harmonic beam, as can be seen from the small Fresnel number, $N_{f}=a^{2} / L \lambda$ [101] between 0.06 and 0.3 


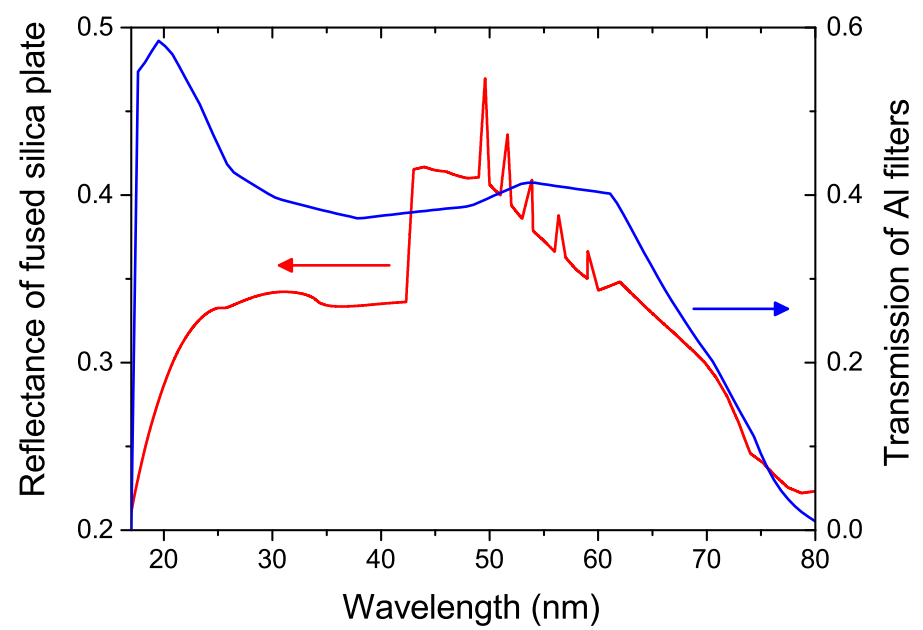

Figure 3.9: Calculated reflectance (red) of the p-polarized harmonic beam between $17 \mathrm{~nm}$ to 80 $\mathrm{nm}$ from an uncoated fused silica plate with an incident angle of $75^{\circ}$ (relative to the normal) and transmission (blue) of the two $200 \mathrm{~mm}$ thick Al filters in series for the same harmonic beam.

calculated for the transmitted wavelength range, where $a \approx 87.5 \mu \mathrm{m}$ (averaged value of the beam radius for two transverse directions) is the focal spot size in the argon jet and $L \approx 1.5 \mathrm{~m}$ is the optical distance from the nozzle to the camera. Together with the optical shutter, single-shot images of the beam profile can be recorded. Fig. 3.10 shows a typical image of a single-shot beam intensity profile. For this particular recording, the drive laser energy is about $3 \mathrm{~mJ}$ and the stagnation pressure is set to $5 \mathrm{bar}$. It can be seen from the two fit curves (red traces) that the beam profile is close to a Gaussian shape in both transverse directions, and that the beam is elliptical, quite comparable with the pump laser ellipticity. For measurements of the harmonic spectrum, home-fabricated transmission gratings $(3,000$ lines $/ \mathrm{mm})$ were used in the harmonic beam path at $6.1 \mathrm{~cm}$ distance in front of the XUV CCD camera. Measurements of average harmonic spectra are usually recorded by integrating over 100 subsequent shots (100 s exposure time) in order to obtain a good signal to noise ratio (typically in the range of 1000). Fig. 3.11 shows a typical example of a captured spectrum comprimising harmonic orders from the $15^{\text {th }}$ up to the $29^{\text {th }}$ order (labelled by red dashed lines). The spectrum was recorded under the same experimental conditions as given above. On the long wavelength side, high diffraction orders including the second order (white dashed lines), even the third order are visible for the shorter wavelengths. The chosen example spectrum is limited to about $53 \mathrm{~nm}$ ( $15^{\text {th }}$ order) due to reabsorption of the generated XUV radiation in argon [102]. We note that the described XUV spectrometer also allows to measure the absolute energy of the harmonics by inserting a calibrated XUV photodiode (AXUV100G, IRD) into the harmonic beam. 


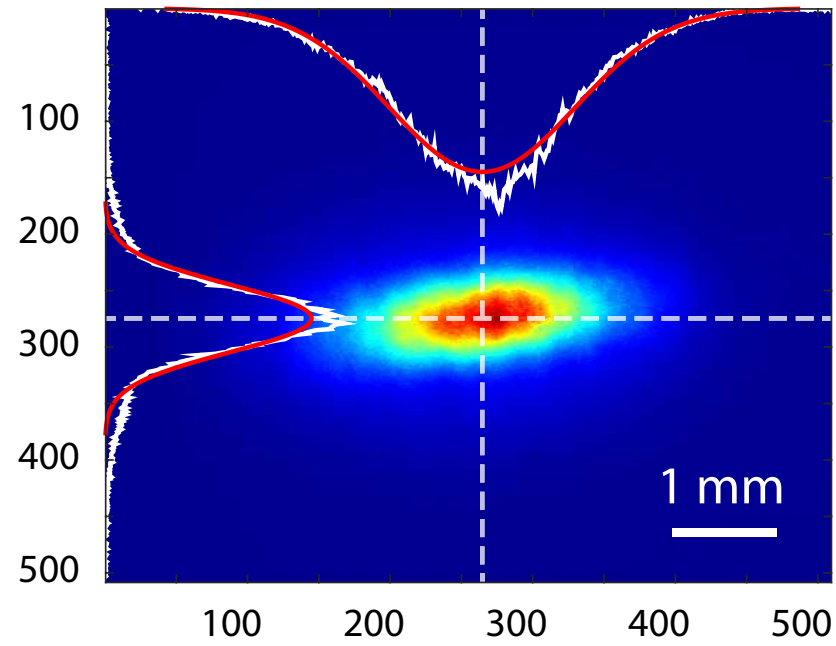

Figure 3.10: Single-shot far-field harmonic intensity profile captured by the XUV CCD camera. The measured beam profiles (white curves) along the respective axes (white dash lines) are plotted, which match well with Gaussian fit curves (red curves). The image was recorded by using a drive laser energy of $3 \mathrm{~mJ}$ and a stagnation pressure of 5 bar. Horizontal and vertical axes are pixels and the color represents counts.

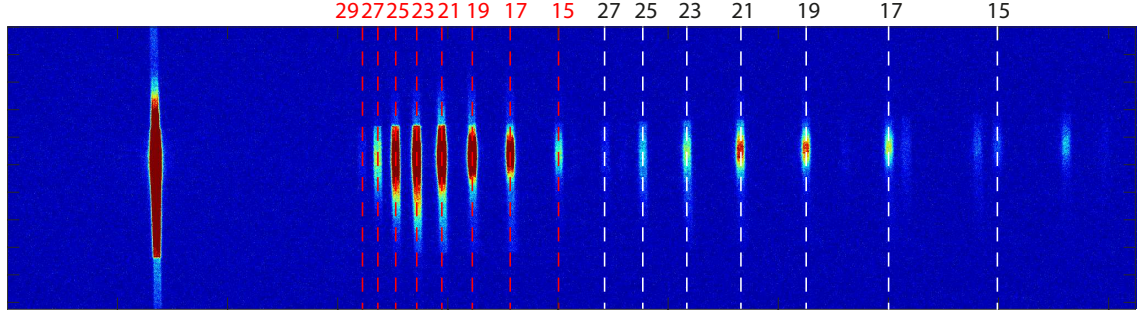

Figure 3.11: Raw CCD data of the harmonic spectrum (integrated over 100 shots) with color presenting counts, and horizontal and vertical axes are pixels. The experiment was performed in the same condition as mentioned in Fig. 3.10. 



\section{4}

\section{REVISITING ARGON CLUSTER}

\section{FORMATION IN A PLANAR GAS JET FOR HIGH-INTENSITY LASER MATTER INTERACTION}

We determine the size of argon clusters generated with a planar nozzle, based on optical measurements in conjunction with theoretical modelling. Using a quasi-one dimensional model for the moments of the cluster size distribution, we determine the influence of critical physical assumptions. These refer to the surface tension depending on the presence of thermal equilibrium, the mass density of clusters, and different methods to model the growth rate of the cluster radius. We show that, in spite of strong variation in the predicted cluster size, $\langle N\rangle$, the liquid mass fraction, $g$, can be determined with high trustworthiness, because $g$ is predicted as being almost independent of the specific model assumptions. Exploiting this observation, we use the calculated value for $g$ to retrieve the cluster size from optical measurements, i.e., calibrated Rayleigh scattering and interferometry. Based on measurements of the cluster size $v$ s. the nozzle stagnation pressure, we provide a new power law for the prediction of the cluster size in experiments with higher values of the Hagena parameter $\left(\Gamma^{*}>10^{4}\right)$. This range is of relevance for experiments on high-intensity laser matter interactions.

\footnotetext{
This chapter has been published as: Y. Tao, R. Hagmeijer, E. T. A. van der Weide, H. M. J. Bastiaens, and K. -J. Boller, Revisiting argon cluster formation in a planar gas jet for high-intensity laser matter interaction, J Appl Phys, 119, 164901 (2016).
} 


\subsection{INTRODUCTION}

The formation of nanometer-sized objects via van-der-Waals aggregation of gas atoms and molecules, called cluster formation, is emerging into a highly important technique for the field of high-intensity laser-matter interactions [103]. As compared to standard gas targets made of atomic or molecular monomers, clusters provide a number of unique advantages and novel options. These are, for instance, achieving higher efficiency in $\mathrm{THz}$ generation [104], high-harmonic generation with shorter cut-off wavelengths, reduced saturation and higher yield [47, 56], increased field gradients for laser-driven particle acceleration [49] or super-hot microplasmas for nuclear fusion [105].

The advantages mentioned are associated with the relatively large mass and size of clusters, typically thousands to millions of atoms or molecules, which offers a much stronger interaction with the drive laser radiation and allows to form spatially tailored density distributions. Specifically, periodic density distributions are promising, such as for direct acceleration of particles [106] or quasi-phase matching in high-harmonic generation [39].

The experimental results obtained with clusters depend, however, critically on the cluster size, $\langle N\rangle$, i.e., on the number of constituent atoms per cluster and, typically, changing global experimental parameters (pressure, temperature) is intended to obtain clusters of a certain size. An example is the retrieval of structural and dynamical information on sub-nanometer and attosecond scales via high-harmonic generation [57]. Another example is the density contrast that can be obtained in periodic structuring of cluster jets, e.g., via an array of wires [50]. Here a lower temperature in the jet increases the cluster size and improves the directedness of the flow, whereas the monomer contribution in the flow gives rise to shock waves that reduce the cluster size and density contrast.

In summary, there is a great need for an easy and reliable method for determining the size of clusters as well as a clear understanding of cluster formation. However, achieving a desired cluster size is problematic. A measurement of the cluster size that has been generated with a certain set of global parameter is difficult due to the highly transient and dynamical character of nanocluster formation [107, 108]. Before we present our measurements and improved theoretical modelling of cluster formation, let us recall the current understanding of cluster formation and according models, and point to some serious limitations.

Generally, clusters are generated in a supersonic jet expansion. The thermodynamic state of the gas in terms of pressure and temperature typically follows a trajectory through the phase diagram as indicated by the red curve in Fig. 4.1, as was already indicated by e.g. Hagena [52]. Starting in the high-temperature, high-pressure region (gas phase) of the gas reservoir, the state initially follows an isentropic change ('dry limit') and then enters the liquid phase region by crossing the saturation curve ('saturation limit') where the pressure, $p$, equals the saturation pressure, $p_{s}$, and nucleation sets in. When the saturation ratio is sufficiently above unity, nucleation reaches its peak value such that the trajectory starts to deviate from the isentropic curve. The temperature reaches a minimum value at the so-called Wilson-point [109] and thereafter increases again due to latent heat release. At this stage, nucleation rapidly decreases and cluster growth takes over as the main condensation process. The thermodynamic state proceeds nearly iso- 


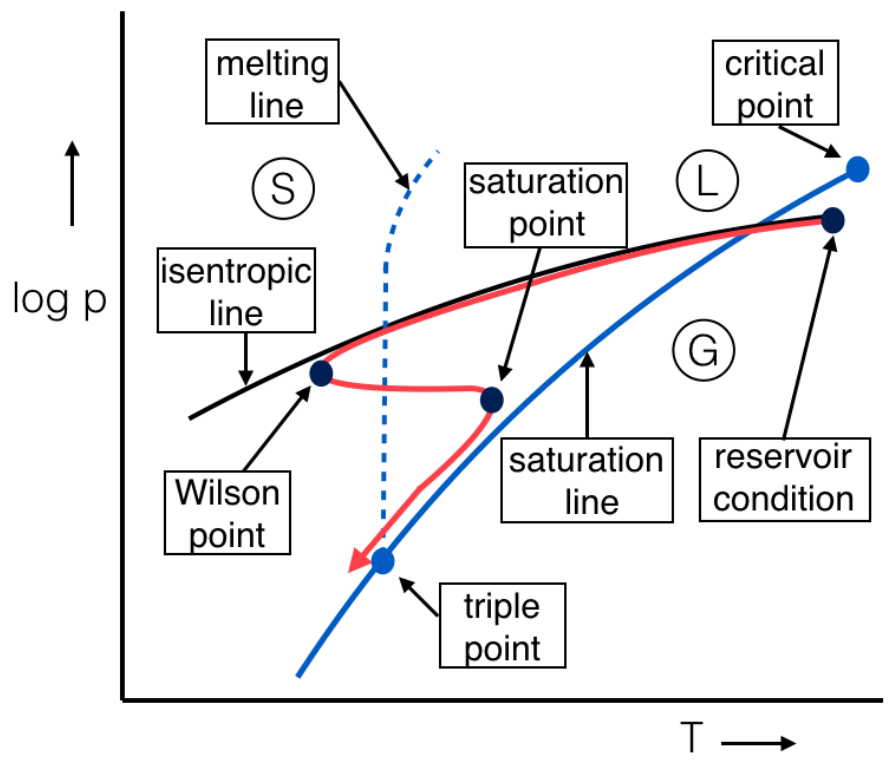

Figure 4.1: Typical vapor expansion trajectory in the phase diagram.

barically towards the saturation curve and temperature reaches a local maximum. From this point onwards pressure remains slightly higher than the saturation pressure while temperature further decreases.

A problem with the described changes of state is that certain details are not known but can have a large influence. For instance, if the trajectory briefly enters the solid state region still one would not expect an instantaneous solidification or even crystallization, but rather that the clusters remain liquid in a super-cooled state. Another issue is the validity of assuming a thermal equilibrium between the forming clusters and the remaining gas. The remaining gas might have a lower temperature (due to expansion) while clusters could show a higher temperature caused by the release of latent heat upon growth. Due to the described phase changes and non-equilibrium dynamics involving nanoscale phenomena, an adequate theoretical description can become extremely complex and suffer from large uncertainties.

Nevertheless, the size of the clusters, $\langle N\rangle$, is widely assumed to follow a simple power law that has been experimentally observed with an orifice [73]:

$$
\langle N\rangle=33\left(\frac{\Gamma^{*}}{1000}\right)^{2.35} .
$$

In this expression, the main experimental conditions are summarized in the Hagena parameter,

$$
\Gamma^{*}=k_{h}\left(\frac{0.74 d}{\tan \alpha}\right)^{0.85} p_{o} T_{o}^{-2.29},
$$

where $k_{h}$ is a gas-specific constant (for argon, $k_{h}=1650$ ), $d$ is the diameter of the orifice 
in $\mu \mathrm{m}, \alpha$ is the expansion half-angle of the jet, $p_{o}$ is the stagnation pressure in mbar and $T_{o}$ is the stagnation temperature in $\mathrm{K}$.

The experimental power law observed at an orifice, however, has only been satisfactorily reproduced with additional experimental data [73] at low gas densities, in a relatively small range of $1000<\Gamma^{*}<7300$ by time-of-flight mass spectroscopy, and only with nozzles having a simple conical symmetry. Nonetheless, the power law is frequently used for evaluating experiments that lie far outside the confirmed range of validity, i.e., at much higher values of the Hagena parameter, also in combination with non-conical geometries. The reason why such extrapolation is still frequently used is that a measurement of $\langle N\rangle$ is actually not trivial, as can be seen as follows.

To obtain experimental information about $\langle N\rangle$, Rayleigh scattering is often applied. However, this method alone does not allow to determine the absolute value of $\langle N\rangle$, because the absolute strength of the Rayleigh scattering signal, $I_{R S}$, is proportional also to the total atomic number density, $n_{a}$, and the liquid mass fraction, $g$, which is the ratio of the number of atoms in the form of clusters to the total number of atoms:

$$
I_{R S} \propto n_{a}\langle N\rangle g .
$$

In some experiments [51], interferometric techniques that yield $n_{a}$ were combined with Rayleigh scattering measurements in order to obtain $\langle N\rangle$ from $n_{a}$ and $I_{R S}$, but this requires additional information on $g$. Often some ad hoc assumptions are made, for instance, by choosing $g=1$ without further justification [55]. Other researchers measured the value of $g$ using Mie scattering from very large clusters (several hundreds of nanometers diameter) $[110,111]$. However, this Mie scattering technique would not be applicable for the much smaller clusters in our work (up to few tens of nanometers diameter).

Recently, Gao et al. [112, 113] have provided the first independent measurement of $g$ via recording time-resolved refractive index changes using frequency domain holography during cluster explosion driven by a high-intensity laser. Their measurements yielded an upper limit for $g$ (e.g. $g<0.5$ at room temperature). Here a problem is that a calculation of the average ionization states of the clusters during laser cluster interaction is needed, the estimation of which is difficult and less reliable.

An interesting alternative that might give more reliable values for $g$ is to develop a physical model based on a description of nucleation and growth of clusters (cluster formation). If the model is sufficiently reliable, calculated values of $g$ can be used in Eq. (4.3) to extract from the experimental results (Rayleigh scattering and interferometry), the absolute values of the cluster size. Boldarev et al. $[74,114]$ have developed a threedimensional model for the investigation of the cluster formation in a conical gas nozzle. They calculated typical values of $g \approx 0.20-0.25$ at room temperature. As an argument for reliability in determination of $g$, they found good agreement of their calculations of $n_{a}$ with the measured values.

Although the described modelling approach seems adequate in those cases, there are also several problems. First of all, the cluster size is extremely sensitive to the surface tension model used. Boldarev et al. [114] applied two different surface tension models in their calculation: the model of Sprow and Prausnitz [115] (which is obtained from experiments), and a linear model which is presented without derivation. Although the linear 
model for surface tension differs only slightly (by 16\%) from the Sprow and Prausnitz model at the triple-point temperature, the predicted cluster size differs at least an order of magnitude.

Secondly, the cluster size is also very sensitive to the liquid mass density which can either be taken from data obtained at saturation conditions [116], or be derived from assuming the solid-like density of a face centered cubic (fcc) lattice [74]. Changing from one model to the other again results in an order of magnitude difference in $\langle N\rangle$.

Thirdly, the cluster size is very sensitive to the growth rate of clusters which is clustersize dependent. In the model applied by Boldarev et al. [114] it is implicitly assumed that $\langle N\rangle$ is equal to the critical cluster size, i.e, the size for which the Gibbs free energy has a maximum. Variation of the growth rate model in this respect has large implications on the resulting values of $\langle N\rangle$.

Finally, the cluster size is very sensitive to the temperature of clusters during the growth phase. One may either assume that the clusters have the same temperature as the surrounding gas whereas, as mentioned above, expansive cooling of the gas and release of latent heat during cluster growth suggest that a thermal non-equilibrium model is to be applied.

These uncertainties show that an experimental determination of the cluster size based on optical measurements requires an improved modelling of cluster formation in search of reliable auxiliary information. Reliable means that this auxiliary information needs to be insensitive to the named variations in modelling as a justification for using it for the evaluation of optical data.

Here, we present a comprehensive modelling of cluster formation and systematically investigate all of the four named sensitivities for the expansion in a supersonic nozzle using argon as an example. Using a model that is based on conservation of mass, momentum and energy, and which describes the cluster size distribution via its moments, we vary the critical model assumptions. These are, specifically, a thermal equilibrium $v s$. non-equilibrium surface tension model, a liquid $v s$. solid-like mass density of the clusters, and a small-cluster and Hill's radius $v s$. large-cluster limit for the growth rate.

In spite of the heavy variations of the cluster size that we observe depending on the model assumptions, we obtain that the liquid mass fraction, $g$, is almost insensitive to the model variations. This strongly supports the validity of our model for a safe determination of $g$. Using this information in combination with new measurements based on Rayleigh scattering and interferometry, we derive a new experimental power law that can be used to determine the cluster size from Hagena parameter, $\Gamma^{*}$. The derived power law complements the prediction of cluster size in the range of at least an order of magnitude higher $\Gamma^{*}$ than before $\left(1.8 \times 10^{4}<\Gamma^{*}<2.5 \times 10^{5}\right)$, i.e., well beyond the proven validity of Hagena's relation.

\subsection{EXPERIMENTS}

To measure the average cluster size, $\langle N\rangle$, we have generated an argon cluster jet using a supersonic slit nozzle (planar nozzle, slit area: $1.0 \times 5.0 \mathrm{~mm}^{2}$, throat size: $220 \times 900$ $\mu \mathrm{m}^{2}$, expansion half-angle: $14^{\circ}$ ) mounted on top of a solenoid pulsed valve (Parker, 9 series). The nozzle is installed in a vacuum chamber and operates at a repetition rate of $0.25 \mathrm{~Hz}$. For Rayleigh scattering and interferometric measurements, we have built an 
experimental setup comprising a Mach-Zehnder interferometer and detection of singlepass Rayleigh scattered light. The detailed description of the experimental setup can be found in Chapter 3.

\subsubsection{INTERFEROMETRY}

To derive the total atomic number density, $n_{a}$, of argon from the experimental data, we first retrieve the phase information from the interference fringe pattern by using spatial Fourier transformation, high-frequency filtering and back Fourier transformation [117]. By comparing the phase information when the gas valve is open $v s$. closed, two-dimensional, cross-sectional phase shift distributions, $\phi$, are obtained. From the phase shift distributions, we calculate the refractive index distribution, $n(\lambda)$, in the cluster jet using

$$
\phi=\frac{2 \pi}{\lambda}(n(\lambda)-1) d_{\mathrm{eff}}
$$

where $\lambda$ is the wavelength of the laser and $d_{\text {eff }}$ is the effective interaction thickness of the cluster jet as experienced by the laser beam. In order to determine $d_{\text {eff }}$, we perform another interferometric measurement of the jet, with the same experimental settings, however, with the nozzle rotated by 90 degrees. The recorded transverse phase shift profile showed a near Gaussian shape having a thickness at half maximum (FWHM) of $d_{\text {eff }}=0.65 \mathrm{~mm}$, as obtained by a Gaussian fit. Replacing the Gaussian shape with a homogeneous (rectangular) density distribution of the same area, and by approximating its width, $d_{\text {eff }}$, with the FWHM from the fit, we obtain $n(\lambda)$ via Eq. (4.4). The total atomic number density, $n_{a}$, is then obtained using the Clausius-Mosotti relation:

$$
n_{a}=\frac{3 \epsilon_{o}}{\alpha(\lambda)}\left[\frac{n(\lambda)^{2}-1}{n(\lambda)^{2}+2}\right]
$$

where $\epsilon_{o}$ is the vacuum permittivity and $\alpha(\lambda)$ is the atomic polarizability of the respective gas. For argon monomers $\alpha(633 \mathrm{~nm})=18.52 \times 10^{-41} \mathrm{Fm}^{2}$, while the polarizability for argon clusters can be computed [74] as $\alpha_{c}=\alpha(\lambda)\langle N\rangle$.

A typical interference fringe pattern obtained from the cluster jet is shown in Fig. 4.2 (a). The presence of the jet can be identified as a slight upward shift of the fringes near the exit of the nozzle as indicated by the arrow. The absolute value of the phase-shift distribution retrieved from the interferogram is presented in Fig. 4.2 (b).

Using Eq. (4.5), we calculate the corresponding total atomic number density, $n_{a}$, and the typical cross sections are shown in Fig. 4.3 where $n_{a}$ is plotted versus lateral position along the nozzle exit about $1 \mathrm{~mm}$ above the exit for various stagnation pressures. It can be seen that the $n_{a}$-profiles are fairly uniform (maximum $\sim 10 \%$ deviation) with steep gradients near the nozzle walls. Increasing the pressure from 50 to 70 bar leads to an increase in average density from $7 \times 10^{18}$ to $10.5 \times 10^{18} \mathrm{~cm}^{-3}$, while the density profile remains of approximately the same shape. The absolute values of the densities are sufficiently high to be suitable for typical laser-cluster experiments such as in highharmonic generation, where comparable densities are used in similar jets $[47,49,56$, $105]$. 
(a)
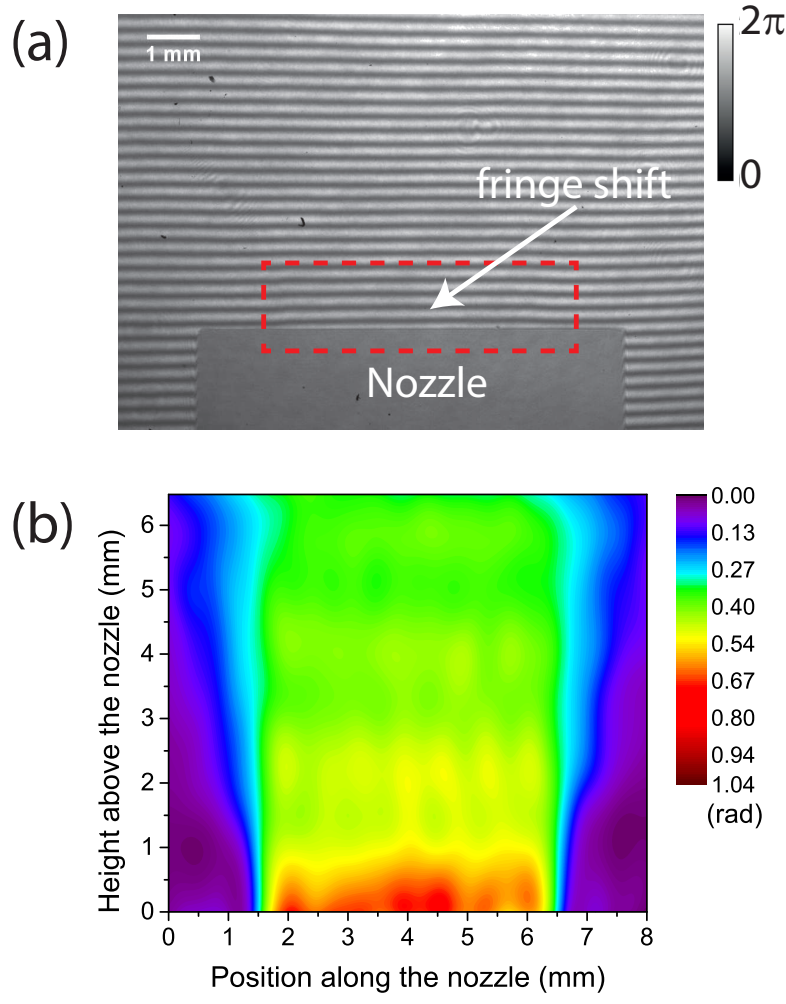

Figure 4.2: Interferogram (a) and retrieved phase shift (b) from an argon cluster jet generated at 50 bar stagnation pressure.

\subsubsection{RAYLEIGH SCATTERING}

In the Rayleigh scattering experiments, we performed a series of measurements with stagnation pressures varying from 8 to 70 bar. To remove a spatially uniform background signal, we define the effective area of the scattering region as the area where the scattering intensity is higher than $3 \%$ of the maximum intensity in the scattering trace [53]. With this definition we observe that the size of the scattering region is close to the length of the slit $(5.0 \mathrm{~mm})$. The Rayleigh scattering intensity at the camera is quantified as a number of counts per pixel measured within the exposure time interval (5 ms). Across the effective area, the number of counts per pixel is spatially integrated to obtain the total Rayleigh scattering signal, $I_{R S}$, which is theoretically equal to [74]

$$
I_{R S}=C \alpha_{R}^{2} P_{o} n_{c}\left\langle N^{2}\right\rangle .
$$

Here $C$ is a constant containing the optical collection angle, the wavelength of the scattered light, the optical system transmission as well as other efficiency parameters of the CCD camera, $\alpha_{R}$ is the atomic polarizability at the used wavelength of $532 \mathrm{~nm}, P_{o}$ is the incident laser power and $n_{c}$ is the cluster density (the number of clusters per unit volume). 


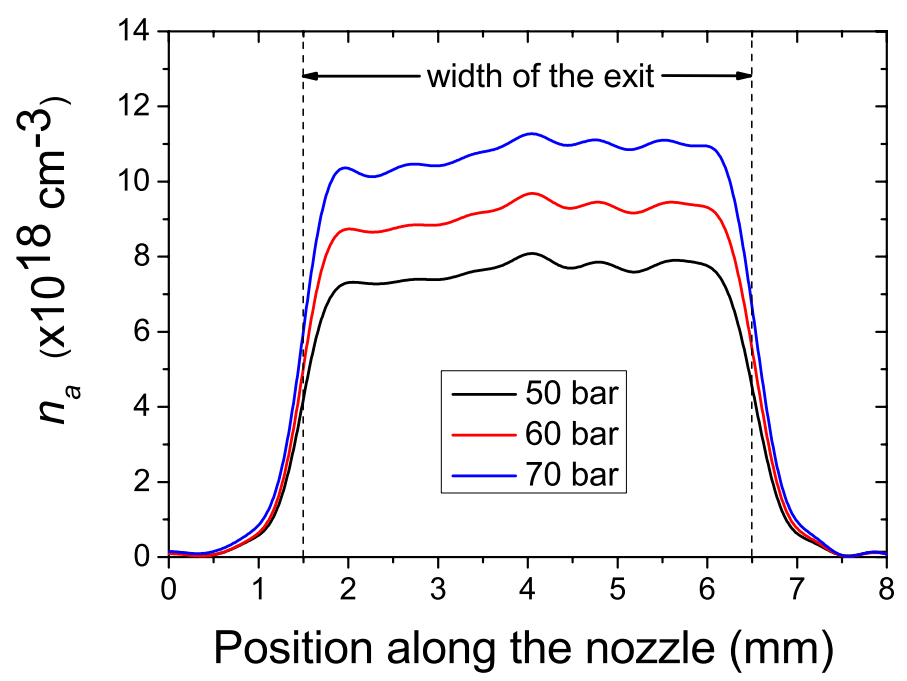

Figure 4.3: Measured spatial profile of the total atomic number density, $n_{a}$, in the argon cluster jet about $1 \mathrm{~mm}$ above the exit of the nozzle for stagnation pressures of 50, 60, and 70 bar, respectively.

Using the conservation law for the number of atoms, $n_{c}\langle N\rangle=g n_{a}$, Eq. (4.6) can be re-written as follows:

$$
I_{R S}=C \alpha_{R}^{2} P_{o} \Psi g n_{a}\langle N\rangle,
$$

where $\Psi=\frac{\left\langle N^{2}\right\rangle}{\langle N\rangle^{2}}=1+\frac{\left\langle N^{2}\right\rangle-\langle N\rangle^{2}}{\langle N\rangle^{2}}$ is the ratio between the averaged squared and the squared average cluster size. By assuming a Gaussian size distribution, Dorchies and co-workers [74, 114] estimated that the value of $\frac{\left\langle N^{2}\right\rangle-\langle N\rangle^{2}}{\langle N\rangle^{2}}$ is typically $20 \%$. In this work, we have used computed values of $\Psi$, obtained from the moments of the cluster size distribution as function of $\Gamma^{*}$. The calculations confirm that $\Psi$ deviates indeed only marginally from unity. For a determination of the factor $C \alpha_{R}^{2} P_{o}$ in Eq. (4.6), i.e, for an absolute calibration of the Rayleigh scattering signal, we make use of the fact that with the vacuum vessel filled with argon gas, the Rayleigh scattering signal amounts to

$$
I_{R S}=C \alpha_{R}^{2} P_{o} n_{a}
$$

Here, we determined $n_{a}$ via a measurement of the gas pressure which yields for the factor in Eq. (4.6) an absolute value of

$$
C \alpha_{R}^{2} P_{o}=\frac{I_{R S}}{n_{a}} .
$$

A typical image of Rayleigh scattering at the argon cluster jet about $1 \mathrm{~mm}$ above the exit of the nozzle is shown in Fig. 4.4. The total Rayleigh scattering signal, $I_{R S}$, from the jet is plotted in the Fig. 4.5 (a) as a function of the nozzle stagnation pressure. In Fig. 4.5 (b) the Rayleigh scattering signal, $I_{R S}, v s$. the static pressure from the calibration experiment in argon gas is plotted. The value of the factor $C \alpha_{R}^{2} P_{o}$ derived from the 


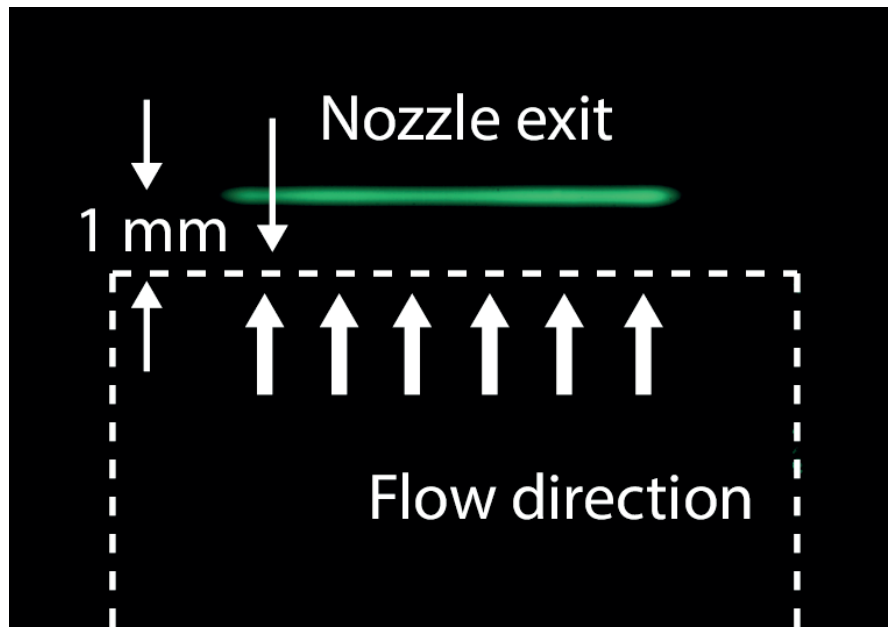

Figure 4.4: A typical Rayleigh scattering image of argon clusters about $1 \mathrm{~mm}$ above the nozzle at a stagnation pressure of 70 bar.

calibration experimental data via a linear fit (red line in Fig. 4.5 (b)) is

$$
C \alpha_{R}^{2} P_{o}=1.32 \pm 0.19 \times 10^{-17} \text { counts } / \mathrm{cm}^{3} \text {. }
$$

Looking at Eq. (4.7), for a determination of the cluster size, $\langle N\rangle$, additional information on the liquid mass fraction, $g$, and the ratio between the averaged squared and the squared average cluster size, $\Psi$, is required. In the following we present a quasi-one di-
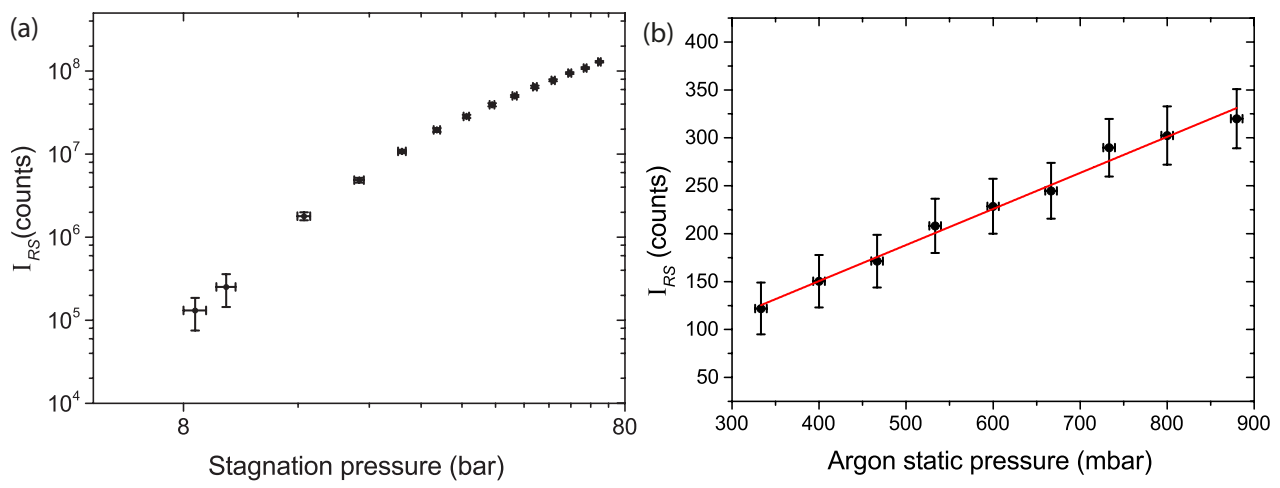

Figure 4.5: Rayleigh scattering signals from the argon cluster jet (a) and from the calibration experiment in static argon gas (b) and the error bars in both figures indicate the standard deviation of the Rayleigh scattering intensity from pixel to pixel for each measurement. The red line is a linear least-square fit to determine the calibration factor $C \alpha_{R}^{2} P_{o}$.

mensional model to calculate $g$ and $\Psi$. Special emphasis is put on an evaluation of the variation of the theoretical predictions when varying the critical physical assumptions. In spite of heavy variations of the cluster size by several orders of magnitude, we observe 
that $g$ remains almost insensitive to variations in the model, which makes it possible to predict $g$ safely.

\subsection{THEORETICAL MODEL}

\subsubsection{NOZZLE GEOMETRY AND RESERVOIR CONDITIONS}

We consider our planar nozzle as depicted in Fig. 4.6 which is described in terms of an axial coordinate $x$, with $x=0$ at the throat, and cross section $A(x)$. The nozzle has a constant width of $w=0.9 \mathrm{~mm}$ along the x axis, a throat height of $h_{o}=0.22 \mathrm{~mm}$, a halfangle of $\alpha=0.244 \mathrm{rad}\left(14^{\circ}\right)$, and a length of $L_{N}=10 \mathrm{~mm}$. The cross section, $A$, is then given by

$$
A(x)=h(x) w, \quad h(x)=h_{o}+2 x \tan \alpha .
$$

The reference reservoir conditions chosen in this work in terms of pressure and temperature are $p_{o}^{\text {ref }}=50 \mathrm{bar}$ and $T_{o}^{\text {ref }}=293 \mathrm{~K}$, respectively. Both parameters have been varied individually while keeping the other variable constant as shown in Table 4.1.

\begin{tabular}{|l|l|}
\hline \multicolumn{2}{|c|}{ pressure series } \\
\hline$p_{o}=10,20,30, \ldots, 100 \mathrm{bar}$ & $T_{o}=T_{o}^{\mathrm{ref}}$ \\
\hline
\end{tabular}

\begin{tabular}{|c|c|}
\hline \multicolumn{2}{|c|}{ temperature series } \\
\hline$p_{o}=p_{o}^{\text {ref }}$ & $T_{o}=200,215,230, \ldots, 350 \mathrm{~K}$. \\
\hline
\end{tabular}

Table 4.1: Definition of the pressure and temperature series.

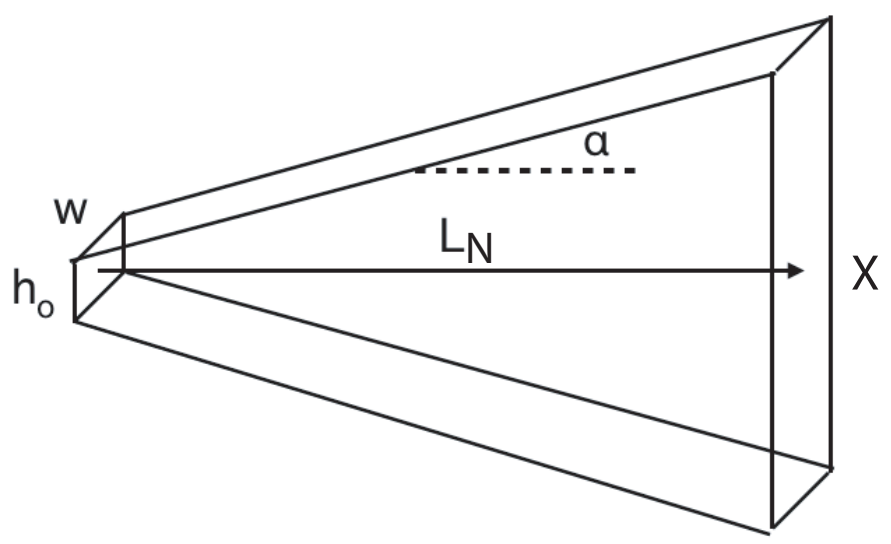

Figure 4.6: Geometry of the planar nozzle used for modelling and in the experiments

\subsubsection{CONSERVATION EQUATIONS}

As we discussed previously (see Section 2.1.2), the conservation equations for mass, momentum and energy in quasi-one dimensional flow with negligible viscosity and heat 
conduction can be written as:

$$
\begin{aligned}
& \frac{D}{D t}(\rho u A)=0, \\
& \frac{D u}{D t}=-\frac{1}{\rho} \frac{D p}{D t},
\end{aligned}
$$

and

$$
\frac{D H}{D t}=0
$$

where $\rho$ and $u$ are the mass density and velocity of the vapor-clusters mixture, $p$ is the vapour pressure, $H$ is the total specific enthalpy and $\frac{D}{D t}$ is the material derivative which in case of stationary flow is defined as

$$
\frac{D}{D t} \equiv u \frac{\partial}{\partial x}
$$

The total specific enthalpy is defined as

$$
H \equiv e+\frac{1}{2} u^{2}+\frac{p}{\rho}
$$

where $e$ is the internal specific energy. Combination of Eqs. (4.13), (4.14) and (4.16) leads to

$$
\frac{D e}{D t}-\frac{p}{\rho^{2}} \frac{D \rho}{D t}=0
$$

which shows that the flow is isentropic in case there is no phase transition.

To relate the thermodynamic variables to each other, we use the thermal equation of state:

$$
p=(1-g) z(\rho, T) \rho R T
$$

and the calorical equation of state:

$$
e=c_{\nu} T+g R T-g L
$$

where $g$ is the liquid mass fraction, $z(\rho, T)$ is the compressibility factor (detailed description is presented in Appendix C, $R$ is the specific gas constant, $T$ is temperature, $c_{\nu}$ is the specific heat at constant volume and $L$ is the latent heat.

To model the formation of clusters as a distribution of clusters of various different sizes, we introduce the distribution function, $f(r)$, where $r$ is the cluster radius. The spatio-temporal development of $f(r)$ is described via its moments which are integrals over the cluster-radius distribution function :

$$
\hat{\mu}_{k}=\mu_{k} / \rho, \quad \mu_{k}=\int_{0}^{\infty} f(r) r^{k} d r .
$$

The moment $\hat{\mu}_{0}$ represents the number of clusters per unit mass and $\hat{\mu}_{3}$ is related to the liquid mass fraction, i.e., the amount of liquid mass per unit mass of the mixture:

$$
g=\frac{4}{3} \pi \rho_{L} \hat{\mu}_{3},
$$


with $\rho_{L}$ the bulk mass density of the liquid phase. Finally, we calculate $\Psi$ from the ratio of moments $\left(\hat{\mu}_{0}, \hat{\mu}_{3}\right.$ and $\left.\hat{\mu}_{6}\right)$ :

$$
\Psi \equiv \frac{\left\langle N^{2}\right\rangle}{\langle N\rangle^{2}}=\frac{\hat{\mu}_{0} \hat{\mu}_{6}}{\hat{\mu}_{3}^{2}} .
$$

The moments satisfy the following transport equations: [118-120]

$$
\frac{D \hat{\mu}_{k}}{D t}=\hat{J} r_{*}^{k}+k\langle\dot{r}\rangle \hat{\mu}_{k-1}, \quad k=0,1,2,3,
$$

where $\hat{J}$ is the classical nucleation rate per unit mass, $r_{*}$ the critical radius, and $\langle\dot{r}\rangle$ a suitable chosen average of the radius-dependent cluster growth rate (see Section 4.3.5). We basically follow the classical nucleation theory as given in, e.g., [121, 122], where the nucleation rate is modeled as

$$
\hat{J}=J / \rho, \quad J=K \exp \left(-\frac{\Delta G_{*}}{k_{B} T}\right) .
$$

Here, $\Delta G_{*}$ is the critical value of the Gibbs free energy of formation,

$$
\Delta G_{*}=\frac{4}{3} \pi r_{*}^{2} \sigma,
$$

with $\sigma(T)$ the surface tension, and $K$ the prefactor:

$$
K=\frac{\rho_{1}^{2}}{\rho_{L}} \sqrt{\frac{2 \sigma}{\pi m_{1}^{3}}},
$$

with $\rho_{1}=(1-g) \rho$ the monomer density, $\rho_{L}$ the bulk density of the liquid phase and $m_{1}$ the monomer mass.

The Gibbs free energy of formation attains its critical value at the critical radius:

$$
r_{*}=\frac{2 \sigma}{\rho_{L} R T \ln S},
$$

where $S$ is the saturation ratio, i.e., the ratio between the vapor pressure $p$ and the saturated vapor pressure $p_{s}(T)$ :

$$
S=\frac{p}{p_{s}(T)}
$$

Finally, the growth rate is modeled by the Hertz-Knudsen growth law:

$$
\dot{r}=\frac{\beta}{\rho_{L}}\left(\frac{p}{\sqrt{2 \pi R T}}-\frac{p_{s, r}}{\sqrt{2 \pi R T_{c l}}}\right),
$$

where $\beta$ is the sticking probability, $T_{c l}$ is the cluster temperature and $p_{s, r}$ is the saturated vapor pressure over a curved surface with radius $r$ :

$$
p_{s, r}=p_{s} \exp \left(\frac{r_{*}}{r} \ln S\right)=p S^{\frac{r_{*}}{r}-1} .
$$

The sticking probability in this work is taken as $\beta=1$. We are aware that this choice somewhat affects the average cluster size and liquid mass fraction, see, e.g., a sensitivity analysis by Sidin et al. [123], but there is a lack of a clear argument to assume another value.

The complete set of equations is solved using the method presented in Appendix C. 


\subsubsection{SURFACE TENSION MODEL}

To model the surface tension for argon, the gas that is used in our experiments, we make use of the model of Sprow and Prausnitz [115]:

$$
\sigma(T)=\sigma_{o}\left(1-\frac{T}{T_{c}}\right)^{c},
$$

where $T_{c}$ is the critical temperature and

$$
\sigma_{o}=37.78 \times 10^{-3} \mathrm{~N} / m, \quad c=1.277 .
$$

We will refer to a first model as the thermal equilibrium surface tension model by assuming that the cluster temperature remains equal to the gas temperature via a sufficiently fast heat transfer.

The critical value of the Gibbs free energy of formation depends cubically on surface tension:

$$
\ln (J / K) \sim \sigma^{3} .
$$

Upon neglecting high order variations of $K$ with $\sigma$ as an approximation, one finds the following estimate for the dependence of the nucleation rate on the surface tension, $\sigma$ :

$$
\frac{J+\Delta J}{J} \approx\left(\frac{J}{K}\right)^{3 \Delta \sigma / \sigma} .
$$

At the nucleation peak one typically has $J / K \approx 10^{-14}$, which, already for a small variation of surface tension, e.g., $\Delta \sigma / \sigma= \pm 10 \%$, leads to a large variation of $(J+\Delta J) / J$ by approximately four orders of magnitude.

A further uncertainty is the following. When pressure and temperature enter the solid phase regime, clusters will tend to change from the liquid phase to the solid phase rendering Eq. (4.31) formally invalid, or the clusters remain in a liquid, super-cooled phase. As we do not have reliable information on the surface tension at that point in the trajectory, here we assume that the clusters remain liquid, and we take the option of a solid state into account in a later step.

In our second model which we refer to as the thermal non-equilibrium surface tension model, we assume that the heat transfer rate between clusters and the gas phase may be relatively low compared to the expansion rate. Below we estimate the relative values of these rates, and the estimation suggests that the clusters have a higher temperature than the surrounding gas phase. To model this thermal non-equilibrium effect to some basic extent the following modification of Eq. (4.31) is proposed.

$$
\sigma(T)= \begin{cases}\sigma_{o}\left(1-\frac{T}{T_{c}}\right)^{c}, & T \geq T_{s}, \\ \sigma_{o}\left(1-\frac{T_{s}}{T_{c}}\right)^{c}, & T<T_{s},\end{cases}
$$

where the saturation temperature $T_{s}(p)$ is implicitly defined by

$$
p_{s}\left(T_{s}(p)\right)=p
$$




\subsubsection{LIQUID MASS DENSITY MODEL}

The critical value of the Gibbs free energy of formation depends inversely quadratically on the liquid mass density:

$$
\ln (J / K) \sim \rho_{L}^{-2}
$$

Upon neglecting again the variation of $K$ with $\rho_{L}$ one finds the following estimate for a variation of the nucleation rate $v s$. a variation of $\rho_{L}$ :

$$
\frac{J+\Delta J}{J} \approx\left(\frac{J}{K}\right)^{-2 \Delta \rho_{L} / \rho_{L}} .
$$

Again at the typical value $J / K \approx 10^{-14}$ with only a small variation of the liquid mass density of $\Delta \rho_{L} / \rho_{L}= \pm 10 \%$ one finds a strong variation of $(J+\Delta J) / J$ by approximately three orders of magnitude.

Two different models for the bulk density are considered. As the first model the density at the saturation line is used [116]:

$$
\rho_{L}^{s}(T)=\rho_{c} \exp \left(\sum_{i=1}^{4} \rho_{i}\left(\frac{T}{T_{c}}\right)^{c_{i}}\right),
$$

where $\rho_{c}$ is the critical density and the coefficients $\rho_{i}$ and $c_{i}$ are given in Table 4.2.

\begin{tabular}{|c|c|c|}
\hline$i$ & $\rho_{i}$ & $c_{i}$ \\
\hline 1 & 1.5004262 & $1 / 3$ \\
2 & -0.31381290 & $2 / 3$ \\
3 & 0.086461622 & $7 / 3$ \\
4 & -0.041477525 & 4 \\
\hline
\end{tabular}

Table 4.2: Coefficients in liquid mass density Eq. (4.39)

To take a possible solidification of clusters into account, we used, as a second model, the fcc packed bulk density of the solid phase [74]:

$$
\rho_{L}^{f c c}=\frac{3}{4} \pi \frac{m_{1}}{v_{o}}, \quad v_{o}=9.0 \times 10^{-30} \mathrm{~m}^{3} .
$$

The ratio of the mass densities as given by the two assumptions is depicted in Fig. 4.7. It can be seen that there is, particularly at the critical temperature, a significant difference in the mass density and, correspondingly, a large difference in nucleation rate might be expected.

\subsubsection{GROWTH RATE MODEL}

The growth rate equation Eq. (4.29) for the radius of a cluster can be rewritten by using Eq. (4.30):

$$
\dot{r}=\frac{\beta}{\rho_{L}}\left(1-S^{\frac{r_{*}}{r}-1} \sqrt{\frac{T}{T_{c l}}}\right) \frac{p}{\sqrt{2 \pi R T}} .
$$




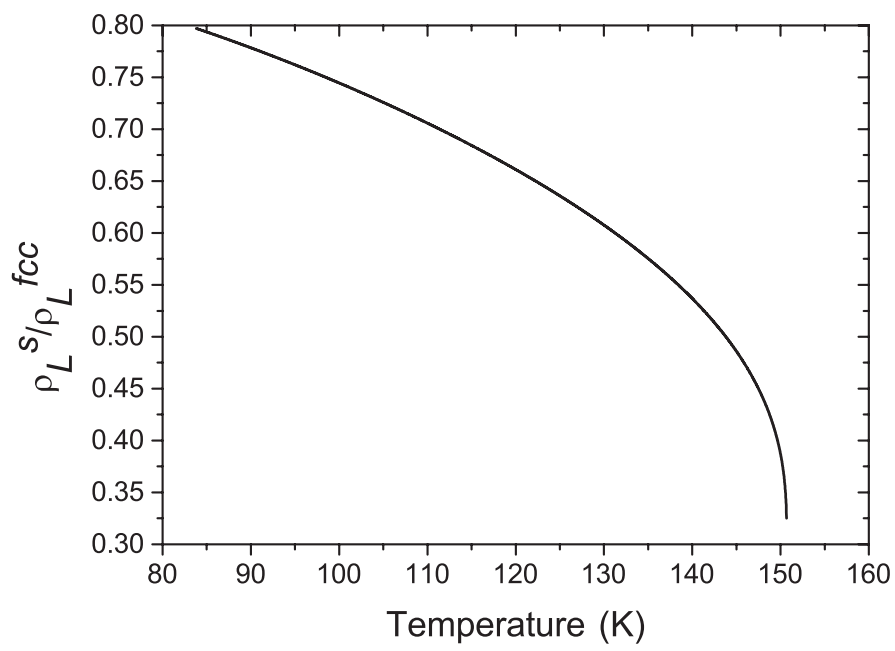

Figure 4.7: Liquid mass density ratio, $\rho_{L}^{s}(T) / \rho_{L}^{f c c}$, as function of temperature, $T$, with $T_{t r}<T<T_{c r}$.

showing that the growth rate depends nonlinearly on the radius and the cluster temperature. An average growth rate, $\langle\dot{r}\rangle$, can be obtained by replacing the cluster radius, $r$, and, the cluster temperature, $T_{c l}$, by suitable average values as follows.

For the cluster temperature, $T_{c l}$, two limiting cases are considered: the thermal equilibrium limit:

$$
T_{c l} \rightarrow T
$$

and the thermal non-equilibrium limit:

$$
T_{c l} \rightarrow T_{s}
$$

where $T_{s}$ is chosen according to the surface tension modification given in Eq. (4.35).

For the average cluster radius, $\langle r\rangle$, three models are considered: the small cluster limit, the so-called Hill's radius, and the large cluster limit. The small cluster limit for thermal equilibrium is defined as

$$
\frac{\langle r\rangle}{r_{*}}=2 \Rightarrow S^{\frac{r_{*}}{r}-1} \rightarrow 1 / \sqrt{S},
$$

and for thermal nonequilibrium as

$$
\frac{\langle r\rangle}{r_{*}}=1 \Rightarrow S^{\frac{r_{*}}{r}-1} \rightarrow 1 .
$$

It is noted that taking $\langle r\rangle / r_{*}=1$ in Eq. (4.44) would lead to a zero growth rate reflecting the maximum in the Gibbs free energy. In the intermediate model by Hill [118] the average radius is taken as Hill's radius:

$$
\langle r\rangle=r_{H} \equiv \sqrt{\frac{\hat{\mu}_{2}}{\hat{\mu}_{o}}},
$$


which means that the average value is obtained from the moments of the calculated size distribution function. The large cluster limit finally is taken as

$$
\frac{\langle r\rangle}{r_{*}} \rightarrow \infty \Rightarrow S^{\frac{r_{*}}{r}-1} \rightarrow S^{-1}
$$

\subsubsection{MODEL VARIATION SENSITIVITY}

The average cluster size, $\langle N\rangle$, is inversely proportional to the liquid mass fraction, $g$ (see Eq.(4.7)). The approach taken in this work is to investigate the sensitivity of the liquid mass fraction with respect to variations of the model. We begin the investigations with a specific choice of model which we call baseline model (Section 4.3.7), because this choice appears to be justified best based on physical arguments. We will show below that both the average cluster size, $\langle N\rangle$, and the cluster number density, $n_{c}$, are extremely sensitive to variation of the model. However, their product, $n_{c}\langle N\rangle=g n_{a}$, turns out to be very insensitive because both $g$ and $n_{a}$ are insensitive, specifically, when the physically more justified thermal non-equilibrium assumption is used.

The average cluster size, $\langle N\rangle$, computed under variation of models as described above is depicted in Fig. 4.8 as a function of the Hagena parameter, $\Gamma^{*}$. The left figure shows the results obtained with the thermal equilibrium models and the right figure shows the results obtained with the thermal non-equilibrium models. It is evident that the results are extremely sensitive to model variations because they lead to differences of up to eight orders of magnitude. This proves that modelling $\langle N\rangle$ using any of the assumptions mentioned will not provide trustworthy predictions. Even when not comparing absolute values of $\langle N\rangle$ but looking only at the variation of $\langle N\rangle$ with $\Gamma^{*}$, rather different trends are predicted by different groups of models. Specifically, the thermal equilibrium models roughly show an opposite trend than non-equilibrium models in that they predict a decreasing cluster size with increasing $\Gamma^{*}$ instead of an increasing cluster size. Such opposite trend can also be observed in the work of Boldarev et al. [114]. Of all thermal non-equilibrium models the model with Hill's radius predicts the largest average cluster size, since it produces the largest growth rate (see Eq. (4.41)).

The corresponding computational results for the liquid mass fraction, $g$, are depicted in Fig. 4.9. Again, the left figure shows the results obtained with the thermal equilibrium models and the right figure with the thermal non-equilibrium models. Variations of the thermal equilibrium models lead to considerable variations in $g$ and even may lead to no nucleation at all (lowest value of $\Gamma^{*}$, small cluster limit with $f c c$-packed density). In contrast, the right figure of Fig. 4.9 reveals that $g$ is very insensitive to variations within all thermal non-equilibrium modelling and Fig. 4.9 is therefore a key figure in this work. The insensitivity of $g$, for thermal non-equilibrium models, together with our time-scale estimation that suggests the presence of thermal non-equilibrium cluster formation (Section 4.3.7), supports the conclusion that our model predicts physically correctly and reliably the liquid mass fraction. This reliability in determining $g$ is what justifies to make use of the calculated $g$-values for deriving the average cluster size from the optical measurements.

The corresponding computational results for the cluster number density, $n_{c}$, are depicted in Fig. 4.10, with again the thermal equilibrium results in the left figure. It can be seen that the sensitivity of $n_{c}$ to model variations is as large as the sensitivity of $\langle N\rangle$. 

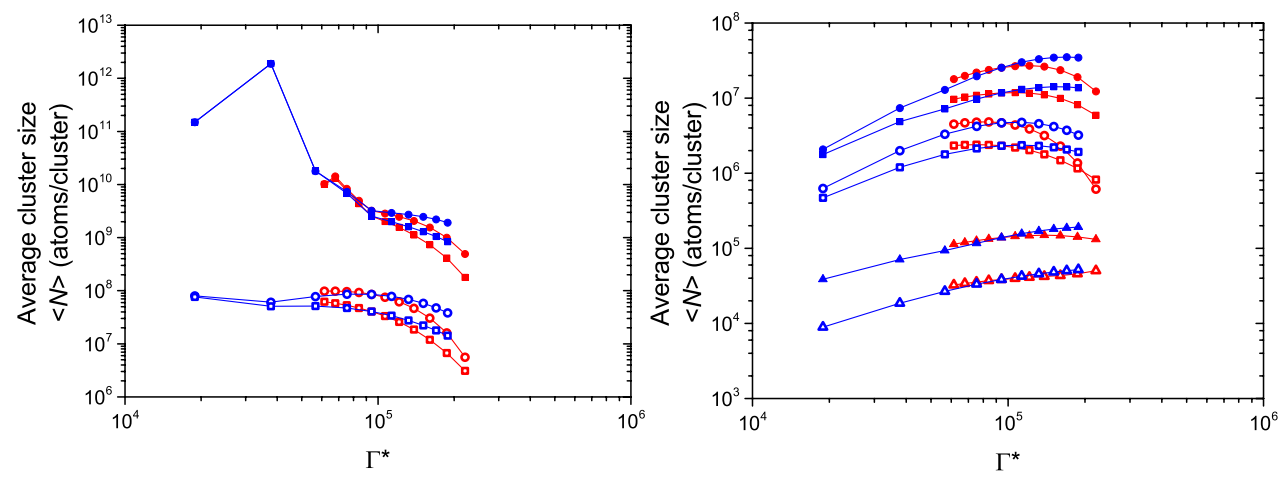

Figure 4.8: Computed cluster size as function of the Hagena parameter at the exit of the nozzle assuming either thermal equilibrium (left, Eq. (4.31) and Eq. (4.42)) or thermal non-equilibrium (right, Eq. (4.35) and Eq. (4.43)) with either super-cooled liquid clusters, $\rho_{L}=\rho_{L}^{s}$ (open symbols), or with perfectly crystalline solid density cluster, $\rho_{L}=\rho_{L}^{f c c}$ (closed symbols), assuming various average radii: $\mathrm{O}:\langle r\rangle=r_{H}, \square:\langle r\rangle=2 r_{*}, \Delta$ : $\langle r\rangle=r_{*}$, either as variation of the nozzle pressure (blue) or as variation of the nozzle temperature (red).
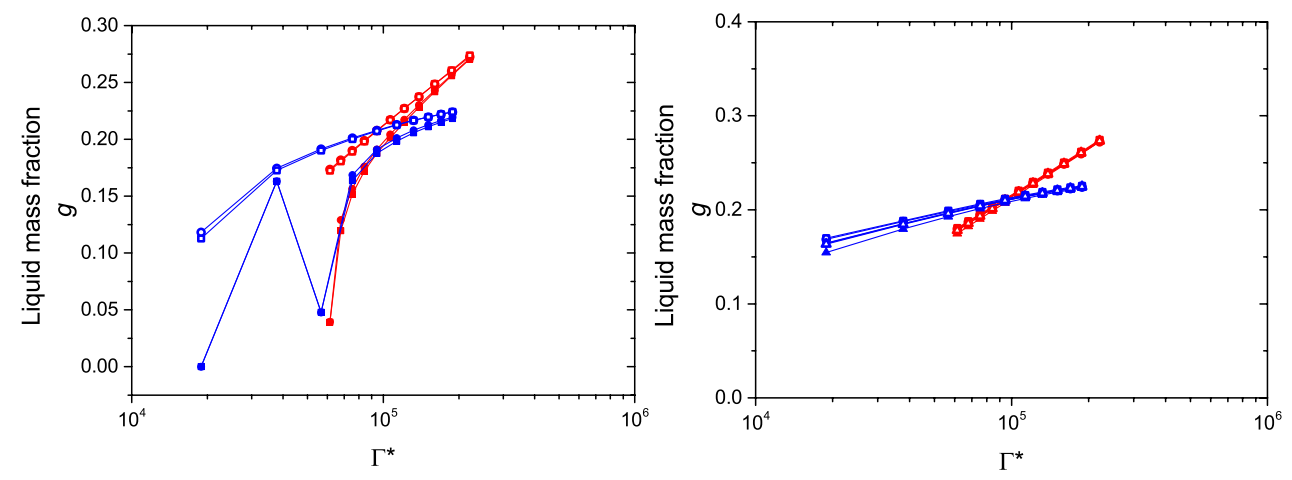

Figure 4.9: Liquid mass fraction as function of the Hagena parameter at the exit of the nozzle assuming either thermal equilibrium (left, Eq. (4.31) and Eq. (4.42)) or thermal non-equilibrium (right, Eq. (4.35) and Eq. (4.43)) with either super-cooled liquid clusters, $\rho_{L}=\rho_{L}^{s}$ (open symbols) or with perfectly crystalline solid density cluster, $\rho_{L}=\rho_{L}^{f c c}$ (closed symbols) with various average radii: $\mathrm{O}:\langle r\rangle=r_{H}, \square:\langle r\rangle=2 r_{*}, \triangle:\langle r\rangle=r_{*}$, and either as variation of the nozzle pressure (blue) or as variation of the nozzle temperature (red).

However, the variations occur in the opposite direction such that the product, $n_{c}\langle N\rangle$, is fairly insensitive, and even very insensitive when only thermal non-equilibrium model variations are considered. This is also reflected in Fig. 4.11 where the computational results for the total atomic number density are depicted, with the thermal equilibrium models in the left figure and the thermal non-equilibrium models in the right figure. Except for the thermal equilibrium small cluster limit with $f c c$-packed liquid mass density all models give near identical results for $n_{a}$. 

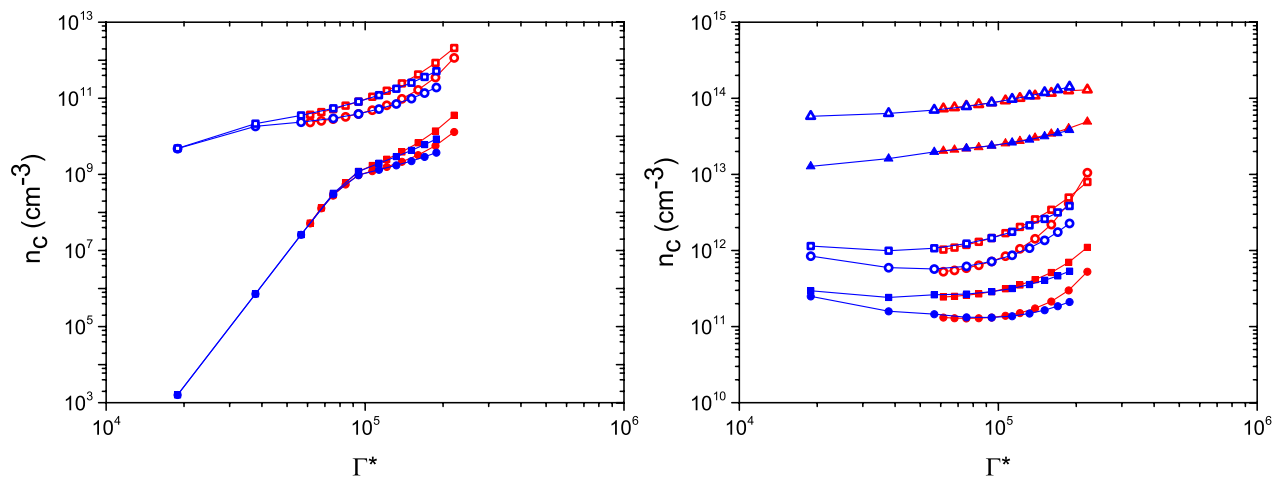

Figure 4.10: Cluster number density as function of the Hagena parameter at the exit of the nozzle assuming either thermal equilibrium (left, Eq. (4.31) and Eq. (4.42)) or thermal non-equilibrium (right, Eq. (4.35) and Eq. (4.43)) with either super-cooled liquid clusters, $\rho_{L}=\rho_{L}^{S}$ (open symbols) or with perfectly crystalline solid density cluster, $\rho_{L}=\rho_{L}^{f c c}$ (closed symbols) with various average radii: $\mathrm{O}:\langle r\rangle=r_{H}, \square:\langle r\rangle=2 r_{*}, \Delta:\langle r\rangle=r_{*}$, and either as variation of the nozzle pressure (blue) or as variation of the nozzle temperature (red).
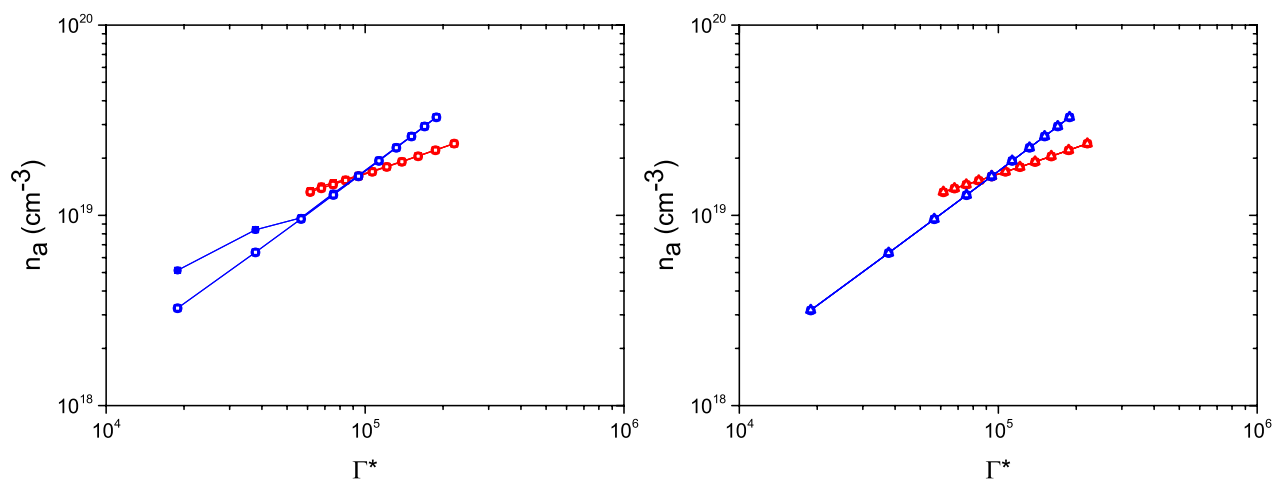

Figure 4.11: Total atom number density as function of the Hagena parameter at the exit of the nozzle assuming either thermal equilibrium (left, Eq. (4.31) and Eq. (4.42)) or thermal non-equilibrium (right, Eq. (4.35) and Eq. (4.43)) with either super-cooled liquid clusters, $\rho_{L}=\rho_{L}^{s}$ (open symbols) or with perfectly crystalline solid density cluster, $\rho_{L}=\rho_{L}^{f c c}$ (closed symbols) with various average radii: $\mathrm{O}:\langle r\rangle=r_{H}, \square:\langle r\rangle=2 r_{*}, \Delta:\langle r\rangle=r_{*}$, and either variation of the nozzle pressure (blue) or as variation of the nozzle temperature (red).

\subsubsection{BASELINE MODEL AND RESULTS}

Among the variation of different choices between possible submodels we have selected a specific model based on physical arguments which we call baseline model. To choose between the thermal equilibrium and nonequilibrium models we compare the thermal relaxation time scale of the clusters, $\tau_{r}$, with the convection time scale of the gas flow, $\tau_{f}$. If the thermal relaxation of the clusters is much slower, this would indicate thermal non-equilibrium. We start the comparison with estimating the impingement rate $v$, i.e., the number of collisions of atoms with a cluster per unit time and per unit area [122]:

$$
v=\frac{p}{\sqrt{2 \pi m_{1} k_{B} T}}
$$


which has the same order of magnitude as

$$
v_{o}=\frac{p_{o}}{\sqrt{2 \pi m_{1} k_{B} T_{o}}}
$$

Assuming, as an approximation, that the condensation rate and evaporation rate on a cluster are proportional to each other, and taking again $\beta=1$, the energy per unit time removed from a cluster is

$$
\dot{e}_{N}=v_{o} A_{N} \frac{3}{2} k_{B}\left(T_{d}-T\right)
$$

where $N$ is the size of the cluster, $A_{N}$ is its surface area and $T_{d}-T$ is the temperature difference between the cluster and the surrounding gas vapor. The thermal relaxation time scale can be now defined as the time needed for $N$ collisions because a number of $\mathrm{N}$ collisions (each collision associated with sticking and reevaporation of one atom) would lead to equal cluster and vapor temperatures:

$$
\tau_{r, N}=\frac{N}{v_{o} A_{N}}
$$

The convection time scale, on the other hand, is taken as the time needed to pass through the nozzle with the speed of sound at stagnation conditions:

$$
\tau_{f}=\frac{L}{\sqrt{\gamma R T_{o}}} .
$$

For the reference conditions already for the smallest clusters predicted by theory, i.e., and $N \approx 10^{4}$ we find that the ratio $\tau_{f} / \tau_{r}$ is rather small on the order of $10^{-3}$. This estimate indicates that thermal relaxation is much slower than convection such that the cluster temperature is expected to be different (higher) than the surrounding vapor temperature. This is why we choose the thermal non-equilibrium options in the surface tension model and in the growth law for our baseline model.

With the same reasoning of relatively slow heat transfer to the clusters, in the baseline model we take the liquid mass density as equal to the saturation liquid mass density instead of the solid-state $f c c$-packed density. In some of the computations the thermodynamic state of the vapor does enter the solid state region but in view of the time needed for clusters to relatively lay over their temperature to that of the vapor we assume that the clusters remain liquid, i.e., liquid mass density is maintained.

The growth law (Eq. (4.41)) is radius dependent and as an approximation we evaluate the growth for a representative average radius. With our model, which provides the moments of the cluster radius distribution, we decided to calculate the average radius from measurements according to Eq. (4.46) (i.e., we calculate Hill's radius). This corresponds to selecting again thermal non-equilibrium model based on quantified physical arguments in order to avoid the less justified assumptions on the cluster size, such as the small or large cluster limits. In summary, our baseline model comprises:

1. thermal non-equilibrium surface tension model, Eq. (4.35),

2. saturated liquid density model, Eq. (4.39),

3. non-equilibrium cluster temperature, Eq. (4.43), and 
4. Hill's average cluster radius, Eq. (4.46).

The results of the baseline model in terms of average cluster size, $\langle N\rangle$, and liquid mass fraction, $g$, are depicted in Fig. 4.12 for the pressure series (blue symbols) and temperature series (red symbols). It is observed that the computed values for the average cluster size from the pressure series and the temperature series have a fair amount of overlap indicating that the Hagena parameter, $\Gamma^{*}$, indeed approximately covers both the dependence on stagnation pressure and the dependence on stagnation temperature. There is overlap at a single point also for the liquid mass fraction but the variation shows two slightly different power laws. The liquid mass fraction varies in between $16 \%$ and $22 \%$ which is consistent with the range reported by Gao et al. [112] and Dorchies et al. [74].
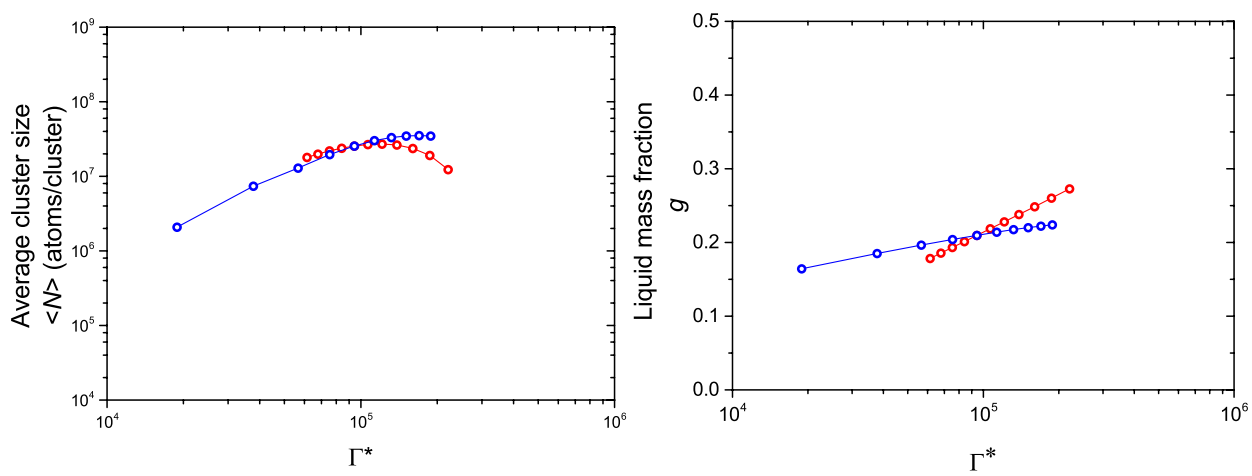

Figure 4.12: Average cluster size and liquid mass fraction computed with the baseline model at constant $T_{o}=$ $T_{\text {ref }}$ and varying $p_{o}$ (blue symbols) and constant $p_{o}=p_{\text {ref }}$ and varying $T_{o}$ (red symbols).

\subsection{AVERage Cluster Size}

By using the liquid mass fraction, $g$, and the ratio between the averaged squared and the squared average cluster size, $\Psi$, as obtained from calculations (using the baseline model) as explained in Section 4.3.7 and shown in Fig. 4.12, an expression for retrieving the average cluster size from optical data can be derived from Eq. (4.6):

$$
\langle N\rangle=\frac{I_{R S}}{C \alpha_{R}^{2} \Psi P_{o} g n_{a}} .
$$

Inserting in this expression the experimentally determined parameters $\left(I_{R S}\left(p_{o}\right), n_{a}\left(p_{o}\right)\right.$ and the calibration factor $\left(C \alpha_{R}^{2} P_{o}\right)$ ), we obtain the relationship between the measured average cluster size, $\langle N\rangle$, and the calculated Hagena parameter, $\Gamma^{*}$. In a double logarithmic plot, which is shown in Fig. 4.13, the measured relation between $\langle N\rangle$ and $\Gamma^{*}$ is plotted as black round symbols. For convenient use in experiments, these data can be represented by a closed algebraic expression obtained by a fit to the data, in the form of a simple power law with a slight linear variation of the exponent.

$$
\langle N\rangle=10^{-2.16}\left(\frac{\Gamma^{*}}{1000}\right)^{7.64-1.60 \log \left(\frac{\Gamma^{*}}{1000}\right)}
$$




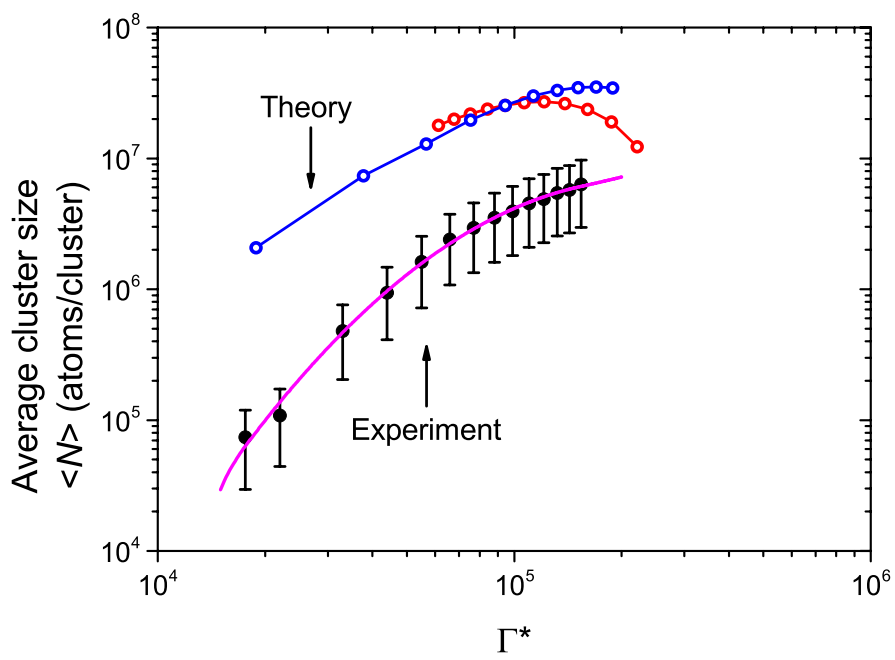

Figure 4.13: Average cluster size from the argon cluster jet $v s$. the Hagena parameter in a double logarithmic plot. The black symbols are derived from optical measurements making use of the calculated, model-insensitive liquid mass fraction, $g$. The error bars are calculated from the error bars obtained in the Rayleigh scattering measurement. The pink solid curve is the corresponding parabolic fit line (Eq. (4.54)). The blue and red symbols are the data calculated from our baseline model.

It can be seen that, besides a relatively small offset (about one order of magnitude), compared to the up to seven orders of magnitude variation calculated with other models, the data are in good agreement with the measurements. Especially, the trends of the experimental values (black symbols) and computed values of $\langle N\rangle v s . \Gamma^{*}$ (blue symbols) are very similar.

\subsection{CONCLUSION}

We have investigated argon cluster formation in a planar nozzle expansion both experimentally and theoretically with a main interest in the average cluster size, $\langle N\rangle$. The average cluster size at a small distance downstream of the nozzle exit has been determined by combining Rayleigh scattering and interferometry data on the one hand and theoretically derived values for the liquid mass fraction on the other hand, as a function of the so-called Hagena parameter, $\Gamma^{*}$.

The baseline theoretical model employed uses a thermal non-equilibrium surface tension model, a saturated liquid density, a non-equilibrium cluster temperature, and Hill's average cluster radius which is computed from the moments of the size distribution. The liquid mass fraction that is obtained from the baseline model proves very insensitive with regards to model variations which justifies the usage of these data to translate measured data to average cluster size. By using our baseline model to calculate the liquid mass fraction, $g$, and using $g$ to retrieve from interferometry and Rayleigh scattering measurements, the average cluster size, $\langle N\rangle$ is determined, in the region of 
higher Hagena parameter, $\Gamma^{*}$, which is well beyond what is known for small cluster from Hagena's guideline [73]. This is summarized in Fig. 4.14 where our experimental data (pink symbols) represented by a modified power law (Eq. (4.54), pink curve) and our theoretical data (blue and red symbols) are plotted for the range of large values $\left(1.5 \times 10^{4}<\Gamma^{*}<2.5 \times 10^{5}\right)$ in the same graph as Hagena's guideline for lower values across the verified region $\left(10^{3}<\Gamma^{*}<7.3 \times 10^{3}\right.$, black line $)$. It can be seen that our power law complements the previously found variation in a consistent fashion and extends the total range of conveniently predictable cluster size from $\Gamma^{*}=10^{3}$ to $2.5 \times 10^{5}$. Thereby the extended range of predictable average cluster size, $\langle N\rangle$, covers values from about a thousand to almost ten million atoms per cluster. Further increasing the detection sensitivity in the optical measurements, such as increasing the laser power to the 10-Watt level seems promising for establishing a unified experimental power law spanning the entire data range. Towards further increased densities, the cluster size appears to approach some limiting size or much slower growth which might be explored with further increased stagnation pressures.

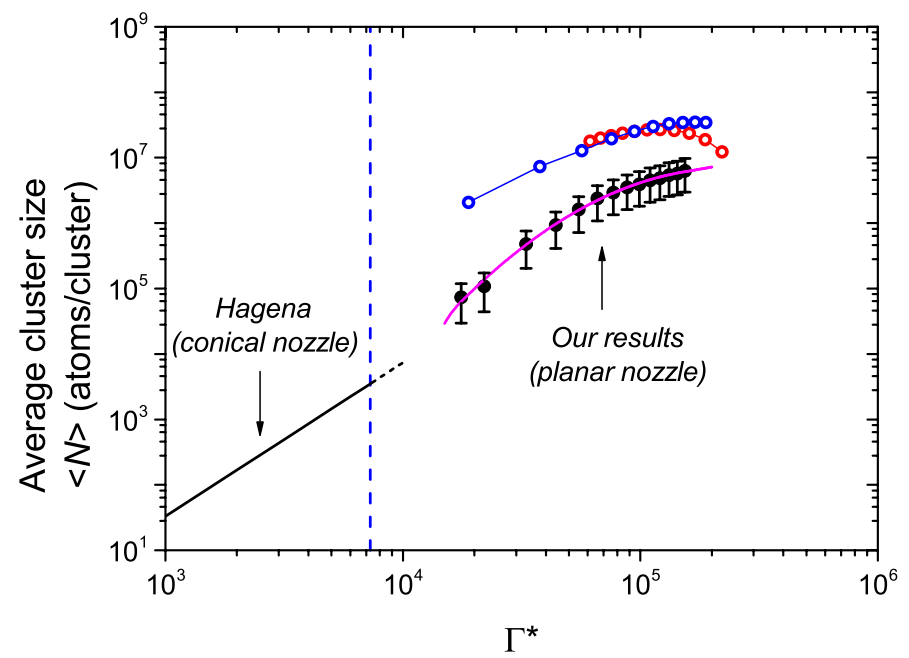

Figure 4.14: Average cluster size from the argon cluster jet at different value of the Hagena parameter in a double logarithmic plot. The black symbols are derived from optical experiments making use of the calculated values of $g$ as summarized by our modified power law (Eq. (4.54), pink curve). The blue and red symbols are the data calculated from our baseline model. In the region of lower $\Gamma^{*}\left(1000<\Gamma^{*}<7300\right)$ the solid line displays Hagena's guideline. 


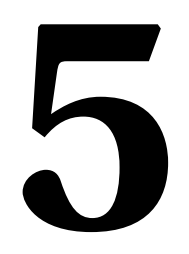

\section{Cluster Size DEPENDENCE OF HIGH-ORDER HARMONIC GENERATION}

We investigate high-order harmonic generation (HHG) from noble gas clusters in a supersonic gas jet. To identify the contribution of harmonic generation from clusters versus that from gas monomers, we measure the high-order harmonic output over a broad range of the total atomic number density in the jet (from $3 \times 10^{16} \mathrm{~cm}^{-3}$ to $3 \times 10^{18} \mathrm{~cm}^{-3}$ ) at two different reservoir temperatures ( $303 \mathrm{~K}$ and $363 \mathrm{~K}$ ). For the first time in the evaluation of the harmonic yield in such measurements, the variation of the liquid mass fraction, $g$, versus pressure and temperature is taken into consideration, which we determine, reliably and consistently, to be below 20\% within our range of experimental parameters. Based on measurements with a thin jet where significant variations in reabsorption and the phase matching conditions can be neglected, we conclude that atoms in the form of small clusters (average cluster size $<1000$ atoms) provide the same higher-order nonlinear response as single-atoms. This implies that HHG in small clusters is based on electrons that return to their parent ions and not to neighbouring ions in the cluster. This conclusion is consistent with the measured harmonic spectra showing no obvious changes of the cut-off wavelength. Our results are in clear contrast to previous work concluding that the single-atom response in small clusters increases with the cluster size, thereby promising a higher output than with monomers. Cluster may still increase the yield of high-order harmonic generation, however, not via the single-atom response but possibly via quasi-phase matching, as the higher mass of clusters allows for a higher density contrast in spatially structuring the nonlinear medium. 


\subsection{INTRODUCTION}

Table-top sources based on high-order harmonic generation (HHG) provide coherent extreme ultraviolet (XUV) radiation on the femtosecond or even attosecond time scale [124]. Such radiation is of great interest for various applications such as probing the ultrafast dynamics of atomic, molecular and solid systems [125], lenless diffractive imaging of objects at the nanoscale [126], as well as seeding free-electron lasers [127]. Typically, noble gas atoms serve as the medium for HHG. On the single-atom level, the mechanism of such a process can be intuitively understood within a simple three-step model [26, 27]: Initially, an electron escapes from its bound state in a strong drive laser field through tunnel ionization. Secondly, the electron is driven away and then accelerated back towards its parent ion. Finally, the electron recombines with its parent ion. However, in spite of progress with phase matching, the macroscopic output remains low [33, 91, 128]. Recently, HHG from crystalline solid materials [129-131] has been discovered and has shown a potential for higher conversion efficiency owing to the high density in solids. In addition, solids can be structured periodically on a micrometer scale which might further enable quasi-phase matching [132]. However, the generation mechanism of HHG in solids differs fundamentally from that in gas atoms. Unlike the atomic three-step model, knowledge of the complex electron dynamics inside the periodic structure of solids, responsible for the generation of harmonics, is missing. Moreover, in order to prevent permanent damage of the crystal, the drive laser intensities in those experiments are at least one order of magnitude lower than those conventionally applied in the gas medium, which results in a rather low cut-off energy [131].

Nanometer-sized clusters, formed via the van-der-Waals aggregation of gas atoms or molecules, provide an attractive alternative for HHG [47, 48, 56, 57, 133, 134] since they combine the low average density of gas and the local high density of solids and liquids. This unique property should allow to investigate the mechanism of HHG across the full range from individual atoms up to solid materials. Furthermore, clusters have been shown as suitable to form spatially tailored density distributions that can be used for direct acceleration of particles [49], an avenue that appears promising also for achieving quasi-phase matching in HHG.

However, the exact mechanism of HHG in clusters is not clear, e.g., it is not known to what extent the simple three-step model remains applicable for describing HHG in clusters. In particular, the recollison mechanism in the three-step model for clusters is not clarified yet. Several reports have attempted to improve the understanding of the mechanism of HHG in clusters based on their more detailed experimental observations. Donnelly et al. [56], Vozzi et al. [47] and Aladi et al. [135] observed an extended cut-off energy and enhanced conversion efficiency in clusters. The results suggest that the electron is initially tunnel ionized from one atom and later recollides with another neighbouring atom (atom-to-neighbour) instead of recombining with its parent atom (atom-to-itself). Such a mechanism would result in the generation of Bremsstrahlung (incoherent, broadband emission). Meanwhile, both Ruf et al. [57] and Park et al. [48] proposed another recollisional mechanism (cluster-to-itself). In that scenario, the harmonic radiation is assumed to be generated from a partially delocalized wave function spreading over the whole cluster. This is supported by measuring the ellipticity as well as the group delay of the high-order harmonics from clusters. Besides, other mechanisms 
might coexist such as resonant heating [136] occuring during the tunnel ionization process, and an alternative recollision mechanism occuring in overdense plasmas [137] or solids [129].

In addition to these microscopic atomic-scale phenomena, HHG is a coherent emission process, such that the yield is also strongly affected by the macroscopic aspect, specifically phase matching, while further modifications can be caused by reabsorption of generated harmonics along the interaction length. Correspondingly, it is not easy to disentangle the single particle (gas monomer or cluster) contribution from an experimental point of view. When attempting a measurement of the intrinsic (microscopic) nonlinear response of clusters versus their size, several considerations are of importance in an experiment. Generally, clusters are produced in a supersonic jet expansion of inert gas atoms. Both the average cluster size and density can be well controlled by the stagnation pressure and reservoir temperature [54, 74]. However, when tuning these two experimental parameters, it is required to carefully keep the measured data, e.g., the generated harmonic order, away from ranges where strong phase mismatch and absorption limit or strongly influence the output signals. This is to ensure that the signals are large enough to be measureable by the detection system [48]. Moreover, except for the average cluster size and density, there is another important parameter, the liquid mass fraction, $g$, which characterizes the formation of clusters. This parameter is defined as the ratio of the number of atoms in the form of clusters to the total number of atoms in the jet. For most of the experiments mentioned above [47, 48, 133, 134], the researchers interpret their results by choosing $g=1$ without further justification, namely, they assume that a pure cluster jet is generated and thereby the measured high-order harmonic $(\mathrm{HH})$ signals are entirely to be attributed to clusters. However, both our recent modelling of cluster formation [138] and other measurements [112,113] strongly indicate that $g$ is not unity but dependent on both the stagnation pressure and reservoir temperature. For instance, the value of $g$ for argon clusters lies only at about $20 \%$ at room temperature over a broad range of stagnation pressures. Even at very low reservoir temperatures $(\sim 173$ $\mathrm{K}$, via cooling by pre-cooled nitrogen gas [113]), $g$ only rises up to $\sim 40 \%$. As a result, the assumption of $g=1$ misleads the interpretation of the measurements. For a valid determination of the nonlinearity of clusters, one has to take into account the contribution to the $\mathrm{HH}$ yield from both clusters as well as gas monomers when interpreting experimental data.

In this work, we present a detailed experimental study on HHG from a supersonic argon jet. To identify a possible dependence of HHG on the average cluster size, we change the stagnation pressure over a broad range to maximize the variation in cluster size. For disentangling the contribution to HHG from clusters and gas monomers, we perform experiments at two different reservoir temperatures in order to vary the liquid mass fraction, $g$, for the same range of cluster sizes. We determine the dependence of the liquid mass fraction, $g$, on both stagnation pressure and reservoir temperature with a high degree of reliability using our one-dimensional model [138]. We find that about a maximum of $20 \%$ of the gas atoms are converted into clusters under our experimental conditions. Combining this value of $g$ with the density dependent measurement of the $\mathrm{HH}$ spectra, we show that small argon clusters below 1000 atoms per cluster have the same single-atom response as gas monomers. For clusters having larger cluster sizes, 
the single-atom response becomes smaller. This result is in contrast to earlier work [48] that concludes an increased nonlinearity of atoms in clusters when $\langle N\rangle$ is smaller than 700 atoms per cluster. Moreover, we observe no changes in the cut-off energy when the average cluster size increases. This confirms other reports [48] suggesting that the collision mechanism described in the three-step model for HHG in gas atoms may still be applicable for HHG in clusters. This means that the tunnelled electron recombines only with its parent ion.

\subsection{EXPERIMENTAL SETUP}

The experimental setup used for HHG in clusters is depicted in Fig. 5.1. Argon clusters are generated from a supersonic slit nozzle (rectangular cross section of exit: $1.0 \times 5.0$ $\mathrm{mm}^{2}$, expansion half-angle: $14^{\circ}$ ) mounted on top of an electro-magnet driven pulsed gas valve (Parker, 9 series) inside a vacuum chamber. The stagnation pressure applied to the valve can be varied between 0 and 70 bar, with an accuracy of 0.2 bar at low pressures ( 0 to 5 bar), and with an accuracy of 0.5 bar in the higher pressure regime ( 5 to 70 bar). With a resistive heater, the temperature of the nozzle can be adjusted between room temperature and $105^{\circ} \mathrm{C}$ with an accuracy of about $0.5^{\circ} \mathrm{C}$. The average cluster size and density, as well as the total atomic number density generated with the supersonic nozzle were measured as a function of stagnation pressure at different temperatures as presented in Chapter 4 [138]. To exclude the influence of changing phase matching conditions in the build-up of the harmonic field, the slit nozzle is oriented perpendicular to the drive laser beam, such that the laser beam propagates through the short dimension (width) of the jet ensuring that the interaction length is shorter than the coherence length. For driving HHG, we employ a femtosecond Ti:Sapphire laser system operating at a center wavelength of $795 \mathrm{~nm}$ at $1 \mathrm{kHz}$ repetition rate (Legend Elite Duo HP USP, Coherent Inc.). The laser generates linearly polarized output pulses with a maximum pulse energy of $6.5 \mathrm{~mJ}$ and a pulse duration of about $40 \mathrm{fs}$ [34]. To avoid any major self-phase modulation and plasma defocusing along the propagation direction, the pulse energy used in our experiment is limited to a fixed value of $3.0 \mathrm{~mJ}$ via a variable attenuator comprising a rotatable half-wave plate followed by a thin film polarizing beam splitter (PBS). The laser pulse is loosely focused about $1 \mathrm{~mm}$ above the nozzle using a lens of $1200 \mathrm{~mm}$ focal length, resulting in a peak intensity of about $1.5 \times 10^{14} \mathrm{~W} / \mathrm{cm}^{2}$ at focus and an effective interaction length [138] of about $650 \mu \mathrm{m}$. The relatively low peak intensity and short pulse duration ensures that the harmonic emission involves only clusters that are not already affected by ionization induced explosion and disintegration, since the time scale for these processes ( $\sim$ hundreds of femtoseconds) is much longer than the drive laser pulse duration ( $\sim 40 \mathrm{fs}$ ) [48]. The generated HH beam co-propagates with the drive laser beam, from which it is primarily separated by an uncoated fused silica plate placed at an incident angle of 75 degree. This incident angle is close to the Brewster angle for the drive laser beam (at center wavelength), such that most of the drive laser beam is transmitted and diverted to an absorbing beam dump. Any residual drive laser radiation that is reflected from the fused silica plate is fully blocked by a set of two 200 -nm thick aluminium (Al) filters placed in series. These filters act as a band pass filter for the harmonic radiation, transmitting more than $40 \%$ in the wavelength range of 17 to $80 \mathrm{~nm}$ [84]. The transmitted $\mathrm{HH}$ beam is detected by an XUV CCD camera (Princeton Instruments, PIXIS- 


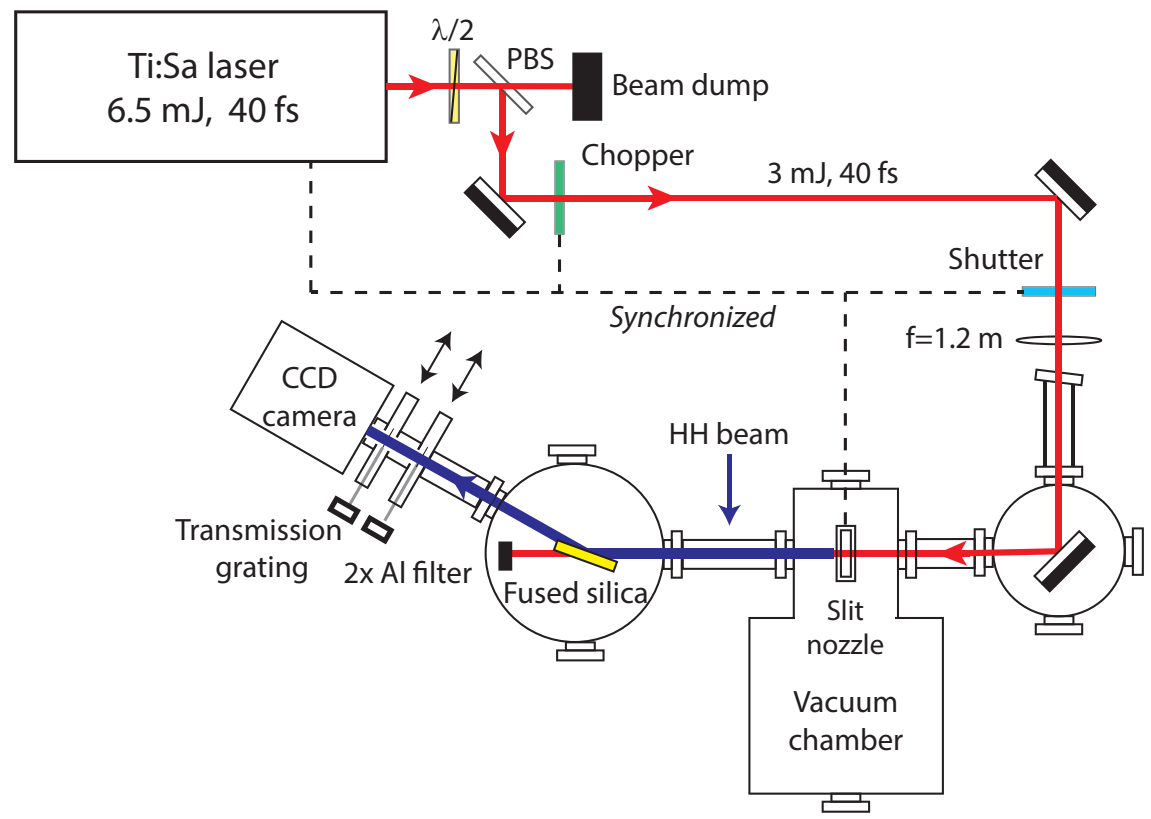

Figure 5.1: Schematic of the experimental setup for HHG in an argon supersonic jet.

XO 2048B) placed behind the filters. For the measurement of the spectral distribution of the $\mathrm{HH}$ output, an in-house fabricated transmission grating (3,000 lines/mm) [84] illuminated through a $300 \mu \mathrm{m}$ slit is shifted into the beam path. To minimize absorption of $\mathrm{HH}$ radiation in the beam path towards the detection system, the pulsed gas valve is operated at a low repetition rate of $1 \mathrm{~Hz}$, as to keep the background pressure below $10^{-3}$ mbar during operation. A mechanical chopper system (MC20008B-EC, Thorlabs Inc) is inserted into the beam path, which reduces the repetition rate of the drive laser from 1 $\mathrm{kHz}$ to $71 \mathrm{~Hz}$, in order to prevent any damage to the fused silica plate and the Al filters due to high average power. For allowing the single-shot detection of the HH beam profile, an additional mechanical shutter (SH05, Thorlabs Inc) could be inserted into the beam path, reducing the repetition rate of the laser further to $1 \mathrm{~Hz}$.

\subsection{Results AND Discussion}

As was emphasized above, it is essential for correct data interpretation that the influence of the liquid mass fraction, $g$, on the $\mathrm{HH}$ yield is clarified, because only this enables us to resolve the relative contribution from clusters and gas monomers. Here, we first determine the dependence of the liquid mass fraction, $g$, on the two main experimental parameters, which are the stagnation pressure, $p_{0}$, and the reservoir temperature, $T_{0}$. An understanding of this dependence is required for the analysis of the scaling of the measured $\mathrm{HH}$ intensity with the stagnation pressure (which determines the atomic number density) as shown below. Next, we discuss the HH spectra and the cut-off wavelength measured for two specific temperatures, at three different stagnation pressures. From 
these spectra, we extract and compare the spectrally integrated $\mathrm{HH}$ signal measured at two different temperatures. Importantly, we show that, in the low-pressure regime, the measured HH signal is not dominated by absorption and phase mismatch which is another essential ingredient for appropriate interpretation of the data. Finally, using a simple model describing the dependence of the harmonic yield on the total atomic number density as well as the average cluster size, we analyze the relative contribution of clusters and gas atoms with regard to the liquid mass fraction. From this, we obtain the singleatom response for clusters with different sizes.

Fig. 5.2 shows the liquid mass fraction, $g$, across a broad range of stagnation pressures, $p_{0}$, (from 300 mbar to $35 \mathrm{bar}$ ), and the two reservoir tempartures, $\left(T_{1}=303 \mathrm{~K}\right.$ and $T_{2}=363 \mathrm{~K}$ ) used in the HHG experiments. To determine $g$, we have used the relation that we derived previously by combining interferometry and Rayleigh scattering data with a theoretical description of cluster formation (see Chapter 4). To allow a direct

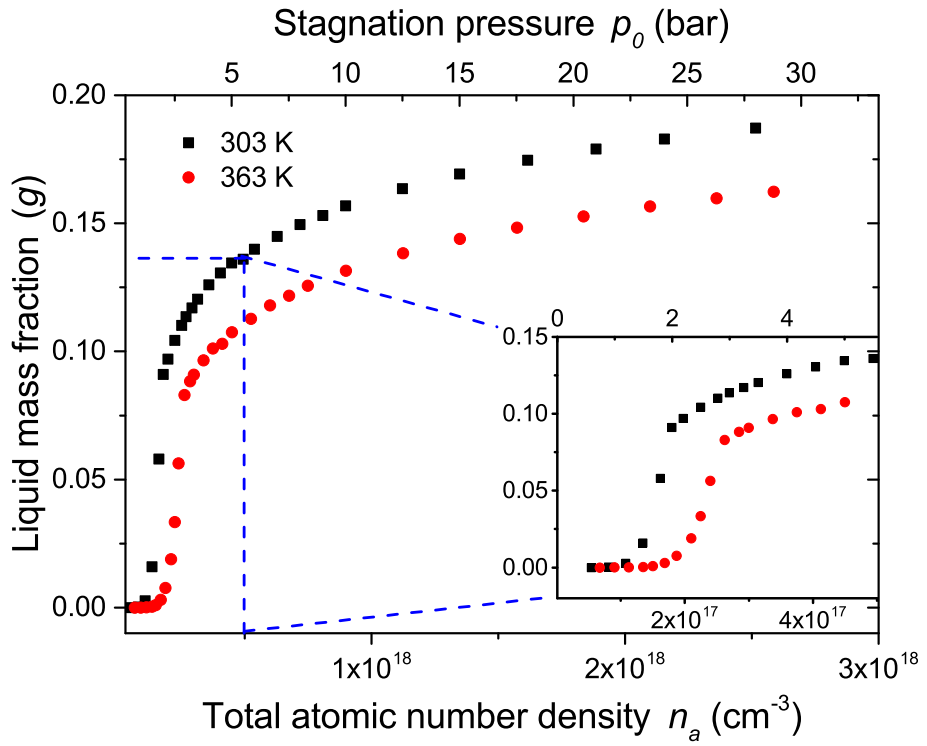

Figure 5.2: Liquid mass fraction, $g$, in the supersonic argon jet as a function of the total atomic number density, $n_{a}$, for two different reservoir temperatures $\left(T_{1}=303 \mathrm{~K}\right.$ and $T_{2}=363 \mathrm{~K}$ ), as obtained from Ref. [138]

comparison of the liquid mass fraction at different temperatures, the stagnation pressure is converted into the total atomic number density, $n_{a} \propto p_{0} / T_{0}$, calibrated by an interferometric measurement [138]. It can be seen that the liquid mass fraction is far off unity in that it grows from extremely small values (near zero) to a maximum of about $19 \%$. The inset shows an enlarged view of the growth of $g$ in the low-density region up to $n_{a}=5 \times 10^{17} \mathrm{~cm}^{-3}$. From Fig. 5.2, it can be clearly seen that, at $T_{1}=303 \mathrm{~K}$ (black squares), the liquid mass fraction is very small $(\leq 0.01)$ for densities up to $n_{a}=10^{17} \mathrm{~cm}^{-3}$, and increases rapidly up to $10 \%$ at a density of about $n_{a}=1.8 \times 10^{17} \mathrm{~cm}^{-3}$. Above this density, $g$ grows more weakly, reaching its maximum value of about $19 \%$ for a density near 
$n_{a}=2.5 \times 10^{18} \mathrm{~cm}^{-3}$. The growth trend of the liquid mass faction at increased temperature, $T_{2}=363 \mathrm{~K}$ (red circles), is very similar, although setting in at a higher density of about $n_{a}=2 \times 10^{17} \mathrm{~cm}^{-3}$. In this case, $g$ reaches a value of $16 \%$ for a density around $n_{a}=2.5 \times 10^{18} \mathrm{~cm}^{-3}$. Within the entire range of the densities and temperatures accessible in our experiment, we find that the liquid mass fraction remains lower than $19 \%$. We note that these rather low values for $g$ correspond well with the experimental results reported by others [113, 139]. Our modelling results show that even at cryogenic temperatures (at $170 \mathrm{~K}$, which can be achieved using pre-cooled nitrogen gas) and high stagnation pressures ( 50 bar) the maximum liquid mass fraction still does not rise above $40 \%$. From this we conclude that all the previous experimental investigations aiming to unravel the size dependent cluster contribution to the $\mathrm{HH}$ yield from the supersonic gas jet have provided questionable conclusions as it is not justified to neglect the major presence of atoms as monomers $[47,48]$.

To study more quantitatively the influence of the liquid mass fraction on the $\mathrm{HH}$ yield, we raise the temperature of the nozzle to decrease $g$, while keeping the total atomic number density in the jet unchanged. This is accomplished by increasing the stagnation pressure such that the ratio $p_{0} / T_{0}$ remains constant. In Fig. 5.3, we present a series of

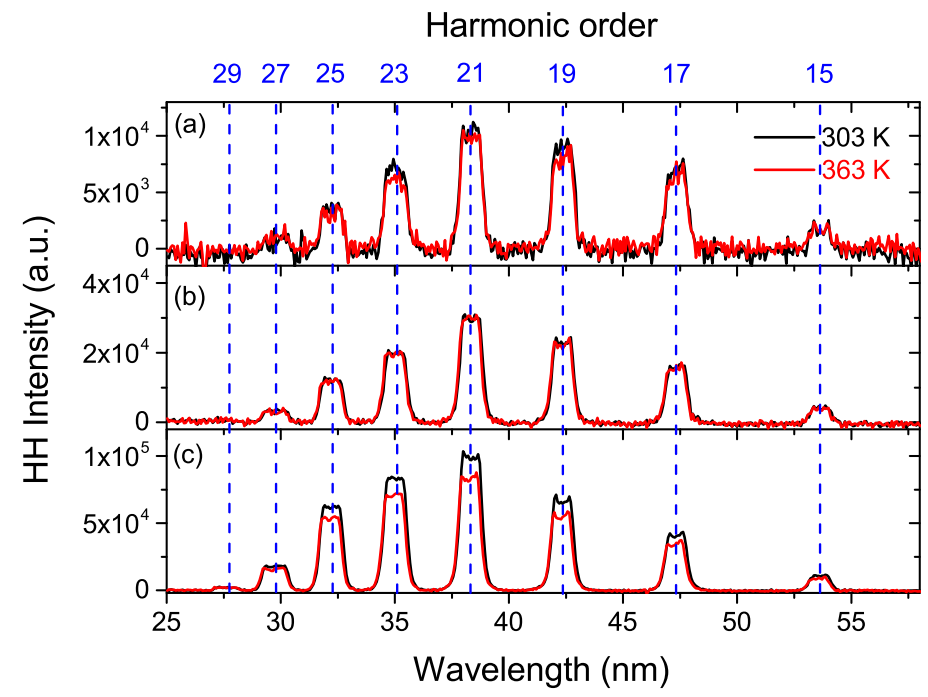

Figure 5.3: $\mathrm{HH}$ spectra measured at $303 \mathrm{~K}$ and $363 \mathrm{~K}$ for the total atomic number densities, $n_{a} \approx 1.6 \times 10^{17}$ (a), $4.5 \times 10^{17}$ (b) and $2.1 \times 10^{18} \mathrm{~cm}^{-3}$ (c). The quasi-flat-top shape of the peaks is caused by the limited resolution of the spectrometer, chosen for maximizing the output signal.

six typical HH spectra measured at two specific temperatures $\left(T_{1}=303 \mathrm{~K}\right.$ and $T_{2}=363$ $\mathrm{K}$ ), for three different total atomic number densities, $n_{a}$, falling within the low (Fig. 5.3 (a)), middle (Fig. 5.3 (b)) and high-density regions (Fig. 5.3 (c)) of the experimental measurement range. Each spectrum is integrated over 100 laser shots to increase the signal to noise ratio as well as reduce the influence due to the fluctuation of the drive laser pulse energy (typically 5\%). For measuring the $\mathrm{HH}$ spectra in the low-density region, 
the density is set to around $1.6 \times 10^{17} \mathrm{~cm}^{-3}$ (with $p_{0}=1.5$ bar at $T_{1}=303 \mathrm{~K}, p_{0}=1.8 \mathrm{bar}$ at $T_{2}=363 \mathrm{~K}$ ). In the middle and high-density regions, the densities are set to around $4.5 \times 10^{17} \mathrm{~cm}^{-3}$ (with $p_{0}=5.0$ bar at $T_{1}=303 \mathrm{~K}, p_{0}=6.0$ bar at $T_{2}=363 \mathrm{~K}$ ) and to around $2.1 \times 10^{18} \mathrm{~cm}^{-3}$ (with $p_{0}=24$ bar at $T_{1}=303 \mathrm{~K}, p_{0}=28$ bar at $T_{2}=363 \mathrm{~K}$ ). From Fig. 5.3, it can be seen that the spectra comprise in total eight harmonic orders, ranging from the $15^{\text {th }}$ to the $29^{\text {th }}$. Note that the $29^{\text {th }}$ harmonic intensity in the low-density region is so weak that it is not observable in the spectrum. Among these harmonic orders, the $21^{\text {st }}$ harmonic consistently exhibits the strongest intensity. Another feature observable in Fig. 5.3 is that the intensity of the harmonics grows with the increasing total atomic number density, from the low-density region to the high-density region. The HH intensity measured at $T_{1}=303 \mathrm{~K}$ is found to be very similar to that measured at $T_{2}=363 \mathrm{~K}$ in both the low and the middle-density regions, while it is slightly higher in the high-density region. Remarkably, we find that the relative shape of the spectra remains identical, independent of the total atomic number density and the temperature. This suggests that the two-field combinations originating from clusters on one hand and gas monomers on the other hand are emitted coherently, i.e., without any change in relative phase, when the density is increased. On the long wavelength side, the spectra are limited to about $53 \mathrm{~nm}\left(15^{\text {th }}\right.$ order). This limit can be traced back to strong reabsorption of the generated XUV radiation in argon [102]. On the short wavelength side, the spectra are limited to about $28 \mathrm{~nm}\left(29^{\text {th }}\right.$ order). This wavelength agrees well with the calculated cut-off wavelength according to the three-step model $\left(\lambda_{\text {cutoff }}=h c /\left(I_{p}+3.17 U_{p}\right) \approx 28 \mathrm{~nm}\right.$, where $U_{p}$ is calculated from the experimental laser parameters). Nevertheless, the measured cutoff wavelengths could imply different mechanisms acting in the recombination process during HHG. Specifically, for HHG in cluster jets, a huge extension of the cut-off wavelength towards shorter wavelengths was previously observed, which has been explained by the so-called atom-to-neighbour collision mechanism [47, 134, 140]. Our observation of the cut-off wavelength does not show such an extension in the measured wavelength range. Instead, the cut-off coincides with the predicted value from the three-step model, as confirmed by experiments of others, suggesting that the coherent emission from clusters is due to the recombination of the tunnel ionized electron with its parent ion within the cluster (atom-to-itself collision mechanism) [48, 57].

Further investigating the high-order harmonic contribution from clusters and gas monomers, we record the harmonic spectra over a broad range of the total atomic number density, $n_{a}$, from $n_{a}=6 \times 10^{16} \mathrm{~cm}^{-3}$ to $n_{a}=2.5 \times 10^{18} \mathrm{~cm}^{-3}$. Since the relative $\mathrm{HH}$ intensity distribution in the measured spectra does not change with density (see Fig. $5.3)$, it allows us to select, as an example, the $21^{\text {st }}$ harmonic $(\approx 38 \mathrm{~nm})$ in the spectra as representative also for the other harmonic orders. The motivation of choosing the $21^{\text {st }}$ harmonic is that it provides the highest signal in all the spectra and therefore provides the highest signal to noise ratio. In Fig. 5.4, we plot the average $21^{\text {st }}$ harmonic yield obtained by spectrally integrating the $21^{\text {st }}$ harmonic signal in the spectra, versus the total atomic number density, $n_{a}$, for two different temperatures in a double logarithmic scale. Due to weak, single-shot signals of the measured HH spectrum, especially for the measurements at low densities, the error bars shown in Fig. 5.4 are derived from measurements of the shot-to-shot fluctuations in the relative harmonic beam energy (in total 100 shots), assuming that the relative error in the average spectral intensity is equal to 


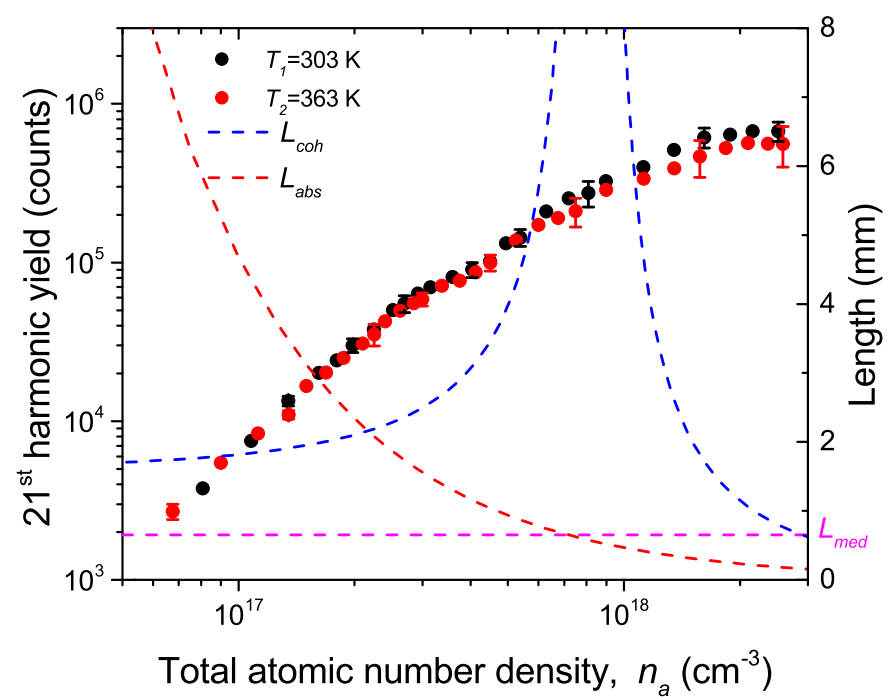

\begin{abstract}
Figure 5.4: Comparison of the average yield (over 100 shots) at the $21^{\text {st }}$ harmonic as a function of the total atomic number density, $n_{a}$, for two different reservoir temperatures, $T_{1}=303 \mathrm{~K}$ (black) and $T_{2}=363 \mathrm{~K}$ (red) respectively. The calculated coherence length (blue dashed curve) and absorption length (red dashed curve) for the $21^{\text {st }}$ harmonic as well as the medium length $\left(L_{\text {med }}=0.65 \mathrm{~mm}\right.$, pink dashed line) are presented for comparison.
\end{abstract}

the relative error in the harmonic beam energy. From Fig. 5.4, it can be seen that the $21^{\text {st }}$ harmonic yield exhibits the same growth for both temperatures, except for some smaller deviation at high densities $\left(n_{a} \geq 7 \times 10^{17} \mathrm{~cm}^{-3}\right)$, that remains within the error margin, where the $\mathrm{HH}$ yield starts to saturate. We also notice that the $\mathrm{HH}$ yield summed up over all harmonic orders in the spectra, i.e., from the $15^{\text {th }}$ harmonic to the $27^{\text {th }}$ harmonic, gives a similar result.

Before conclusions can be drawn on the single-particle (clusters or gas mono-mers) contribution to the $\mathrm{HH}$ yield, the influence of the absorption as well as the phase mismatch should be minimized. For finding the appropriate experimental density range, we plot the absorption length ( $L_{a b s}$, red dashed curve) and the coherence length $\left(L_{c o h}\right.$, blue dashed curve) in Fig. 5.4 versus the atomic number density for comparison with the effective experimental interaction length (effective length of the medium, $L_{m e d}=0.65$ $\mathrm{mm}$, pink dashed line as determined in Chapter 4).

The absorption length, starting with a rather big value, $L_{a b s}=6.5 \mathrm{~mm}$, drops gradually with increasing total number density, $n_{a}$. The length becomes smaller than the length of the medium at higher densities $\left(n_{a} \geq 7 \times 10^{17} \mathrm{~cm}^{-3}\right)$, which means that here the measured $21^{\text {st }}$ harmonic yield is mainly limited by reabsorption in the jet and, therefore, may mask the behaviour of the cluster size. To ensure that the influence of absorption on $\mathrm{HH}$ yield can be neglected at the lower densities below $n_{a}=7 \times 10^{17} \mathrm{~cm}^{-3}$, we checked 
the $\mathrm{HH}$ yield for all different harmonic orders, and found no obviously different growth trend below $n_{a}=7 \times 10^{17} \mathrm{~cm}^{-3}$ among the different harmonics. Such similarity in the growth trend indicates that here the absorption does not play an important role for the $\mathrm{HH}$ yield. As the absorption length is strongly wavelength dependent, the yield should also become strongly dependent on the harmonic orders (with the largest difference between the $15^{\text {th }}$ harmonic and the $27^{\text {th }}$ harmonic) if absorption would play a role.

The coherence length, $L_{c o h}$, is calculated from the wave-vector mismatch consisting of the atomic, the plasma, and the geometric dispersion (see Chapter 2). It can be noticed that the $L_{c o h}$ remains at least three to four times longer than $L_{\text {med }}$ for a broad range of densities. In the range from $n_{a}=5 \times 10^{16} \mathrm{~cm}^{-3}$ to $n_{a}=3 \times 10^{17} \mathrm{~cm}^{-3}$, the wave-vector mismatch is mainly dominated by the geometry dispersion originating from the Gouy phase shift, which is independent of $n_{a}$. Further increasing $n_{a}, L_{c o h}$ grows rapidly and reaches its maximum value around $n_{a}=8 \times 10^{17} \mathrm{~cm}^{-3}$ where the minimum wave-vector mismatch is achieved. Below $n_{a}=8 \times 10^{17} \mathrm{~cm}^{-3}$, a maximum variation of $10 \%$ in the $\mathrm{HH}$ output is theoretically expected when phase mismatch is taken into consideration in the density range around $n_{a}=7 \times 10^{16} \mathrm{~cm}^{-3}$. For $n_{a}>8 \times 10^{17} \mathrm{~cm}^{-3}, L_{c o h}$ drops dramatically due to the dispersion of a large density of ionized electrons and becomes even shorter than $L_{\text {med }}$ around $n_{a}=2.5 \times 10^{18} \mathrm{~cm}^{-3}$, i.e., here the $\mathrm{HH}$ yield is strongly influenced by phase mismatch such that the high-pressure range cannot be analyzed straightforwardly for a cluster-size dependent atomic nonlinearity. For a convincing evaluation without significant influence of absorption and phase mismatch, we limit ourselves to the measured $\mathrm{HH}$ yield at densities below $n_{a}=7 \times 10^{17} \mathrm{~cm}^{-3}$. Here the measured $\mathrm{HH}$ yield is dominated by the single-atom response of single particles.

For deriving the relative single-atom response from the clusters and gas monomers, we propose a simple model which includes the liquid mass fraction, $g$, to interpret the dependence of the $\mathrm{HH}$ yield on the total atomic number density, $n_{a}$. The liquid mass fraction, $g$, was misrepresented in previous experiments by simply assuming $g=1$. Instead, $g$ is essential for evaluating the harmonic contribution from clusters and cannot be generalized as single representing value. The value of $g$ determines the fraction of atoms in the form of differently sized clusters with an average size, $\langle N\rangle$, that may generate harmonics with a different efficiency than the efficiency of the gas monomers, and therefore $g$ affects the $\mathrm{HH}$ yield. In other words, if atoms provide a different yield, depending on whether they are part of a cluster or not, the HH output will be different when changing $g$ while maintaining $n_{a}$ constant. In our model, we follow the basic approach of Durfee et al. and Constant et al. [91, 102]. Here the generated harmonic field from gas monomers or individual atoms which form clusters scales proportionally with the harmonic dipole amplitude and with the atomic number density, when absorption and phase matching terms can be neglected. Consequently, the generated $q^{\text {th }}$ harmonic field contributions from gas monomers, $E_{\text {mono }}(q)$, and from clusters, $E_{c l}(q)$, can be written as follows:

$$
\begin{gathered}
E_{\text {mono }}(q) \propto d_{\mathrm{mono}}^{q} \cdot n_{\text {mono }}, \\
E_{c l}(q) \propto d_{\mathrm{cl}}^{q}(\langle N\rangle) \cdot n_{c l},
\end{gathered}
$$

where $d_{\text {mono }}^{q}$ and $d_{\mathrm{cl}}^{q}(\langle N\rangle)$ represents the $q^{\text {th }}$ harmonic dipole amplitude induced in gas monomers and in the individual atoms inside clusters with an average cluster size, $\langle N\rangle$. 
$n_{m o n o}$ and $n_{c l}$ are the atomic number densities for gas monomers and clusters, respectively. By introducing the liquid mass fraction, $g$, these two terms can be described in terms of the liquid mass fraction, $g$, and the total atomic number density, $n_{a}$, as: $n_{\text {mono }}=[1-g(\langle N\rangle, T)] n_{a}$, and $n_{c l}(\langle N\rangle)=g(\langle N\rangle, T) n_{a}$. With these parameters, Eqs. (5.1) and (5.2) can be re-written as:

$$
\begin{gathered}
E_{\text {mono }}(q) \propto d_{\text {mono }}^{q}[1-g(\langle N\rangle, T)] n_{a}, \\
E_{c l}(q) \propto d_{\mathrm{cl}}^{q}(\langle N\rangle) g(\langle N\rangle, T) n_{a} .
\end{gathered}
$$

Assuming that the generated harmonic field by gas monomers and clusters add up coherently, the measured $\mathrm{HH}$ yield, $\xi(q)$, will scale with the square of the sum of both fields, which can be expressed as

$$
\begin{aligned}
\xi(q) & \propto\left[E_{\text {mono }}(q)+E_{c l}(q)\right]^{2} \\
& =C\left[d_{\text {mono }}^{q}(1-g(\langle N\rangle, T))+d_{\mathrm{cl}}^{q}(\langle N\rangle) g(\langle N\rangle, T)\right]^{2} \cdot n_{a}^{2},
\end{aligned}
$$

where $\mathrm{C}$ is a wavelength dependent calibration factor, that takes into account the efficiency with which the harmonic radiation emitted from the jet reaches the XUV CCD camera, including the reflectance of the fused silica plate, the transmission of the Al filters and the grating, as well as the responsivity of the camera.

To determine the relative single-atom response for gas monomers and clusters using our model, we re-plot from Fig. 5.4, the $\mathrm{HH}$ yield for the $21^{\text {st }}$ harmonic for two different temperatures separately in Fig. 5.5 (a) and (b) versus the total atomic number density (lower axes) as well as versus the average cluster size (upper axes). The average cluster size, $\langle N\rangle$, that corresponds to the total atomic number density, $n_{a}$, is displayed on the top axis of both figures. $\langle N\rangle$ is determined from the stagnation pressures, $p_{0}$ and the two specific temperatures, $T_{1}=303 \mathrm{~K}$ and $T_{2}=363 \mathrm{~K}$, using Hagena's law (for $\langle N\rangle\langle 1000$ ) and a new developed power law (for $\langle N\rangle \geqslant 1000$ ) as mentioned in Chapter 4 . The calculated liquid mass fraction, $g$, for both temperatures is also plotted for comparison. It can be seen that, for both temperatures, the $\mathrm{HH}$ yield initially grows rather rapidly, up to $n_{a}=3 \times 10^{17} \mathrm{~cm}^{-3}$, where $\langle N\rangle$ is around 1000 atoms per cluster. Specifically, the growth of the $\mathrm{HH}$ yield for both temperatures can be fitted, on the doublelogarithmic scale of the graph, by the same linear relation (red solid line) with a slope of $2 \pm 0.05$ and the same interception on the vertical axis of the graphs at $-30.1 \pm 0.05$. Since the slope has a fitted value of two with a rather small error, we conclude that, simply, the $\mathrm{HH}$ yield scales with the square of the total atomic number density, namely, $\xi(\mathrm{HH} 21) \propto \mathrm{n}_{\mathrm{a}}^{2}$. According to our model, such a quadratic growth implies that the term $C\left[d_{\mathrm{cl}}^{\mathrm{HH} 21}(\langle N\rangle) g(\langle N\rangle, T)+d_{\mathrm{mono}}^{\mathrm{HH} 21}(1-g(\langle N\rangle, T))\right]$ in Eq. 5.5, is independent of $n_{a}$, and remains the same at two different chosen temperatures $\left(T_{1}\right.$ and $\left.T_{2}\right)$. Since we keep all the experimental settings the same, including the exposure time and gain setting of the camera when recording the spectra at different temperatures, the calibration factor $C$ remains unchanged. Therefore a rather simple relation for the two temperatures can be derived as follows

$$
\left[d_{\mathrm{cl}}^{\mathrm{HH} 21}(\langle N\rangle)-d_{\mathrm{mono}}^{\mathrm{HH} 21}\right] g\left(\langle N\rangle, T_{1}\right)=\left[d_{\mathrm{cl}}^{\mathrm{HH} 21}(\langle N\rangle)-d_{\mathrm{mono}}^{\mathrm{HH} 21}\right] g\left(\langle N\rangle, T_{2}\right) .
$$


Average cluster size, $\langle N\rangle$

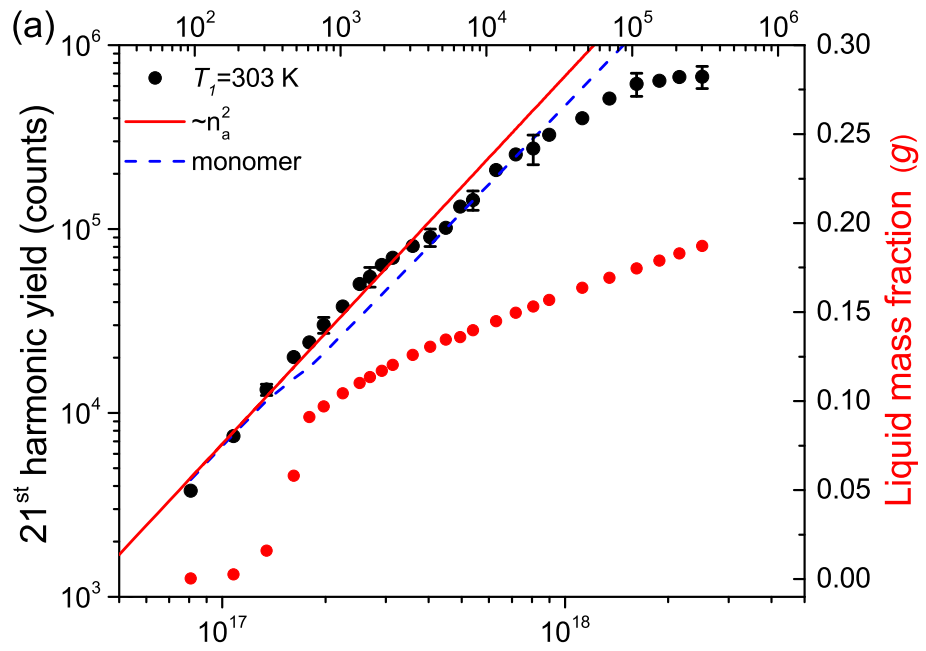

Total atomic number density, $n_{a}\left(\mathrm{~cm}^{-3}\right)$

Average cluster size, $\langle N\rangle$

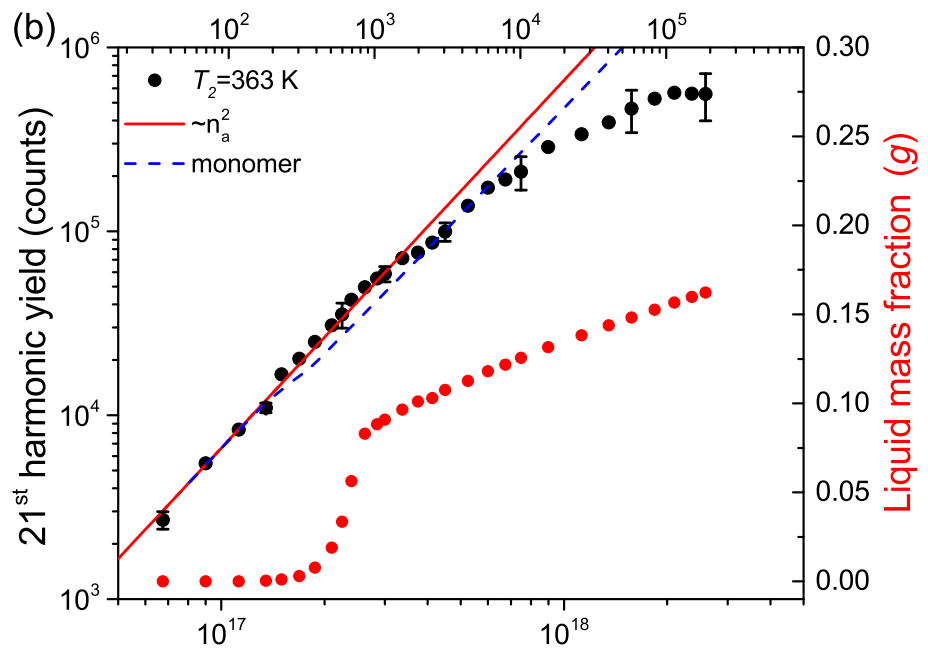

Total atomic number density, $n_{a}\left(\mathrm{~cm}^{-3}\right)$

Figure 5.5: Detailed plot of the $21^{\text {st }}$ harmonic yield versus the total atomic number density, $n_{a}$ at two different temperatures $\left(T_{1}=303 \mathrm{~K}\right.$ (a) and $T_{2}=363 \mathrm{~K}$ (b)). The corresponding average cluster size, $\langle N\rangle$, is displayed at the top axes. The liquid mass fraction, $g$, is plotted in both figures as well. The red solid lines show a $\xi_{\mathrm{HH} 21} \propto n_{a}^{2}$ relation. For comparison, the blue dashed lines show the $21^{\text {st }}$ harmonic yield generated only from the gas monomers. 
In this relation, $d_{\mathrm{cl}}^{\mathrm{HH} 21}(\langle N\rangle)$ and $d_{\mathrm{mono}}^{\mathrm{HH} 21}$ are intrinsic properties of the particles and therefore are independent of the stagnation pressure and the reservoir temperature. The liquid mass fraction, $g$, as shown in Fig. 5.5, however, remains varying with the stagnation pressure and the reservoir temperature and, importantly, has a different dependence on $n_{a}$ and $\langle N\rangle$ for the two temperatures. This means that for the same $\langle N\rangle$, at different temperatures, e.g., $T_{1}$ and $T_{2}, g\left(\langle N\rangle, T_{1}\right) \neq g\left(\langle N\rangle, T_{2}\right)$. As a result, Eq. (5.6) yields that $d_{\mathrm{cl}}^{\mathrm{HH} 21}(\langle N\rangle)=d_{\mathrm{mono}}^{\mathrm{HH} 21}$. This solution leads to the conclusion that below $n_{a}=3 \times 10^{17} \mathrm{~cm}^{-3}$, these clusters with average cluster sizes, $\langle N\rangle$, up to $\sim 1000$ atoms per cluster can be treated as an ensemble of independent atoms in terms of the $\mathrm{HH}$ yield that they generate. In other words, these atoms inside small clusters provide the same single-atom nonlinear response as the gas monomers, resulting in the same harmonic generation efficiency. Note that we do not observe a faster growth trend than the quadratic growth for the $21^{\text {st }}$ harmonic yield with $\langle N\rangle$ up to 700 atoms per cluster. This is in clear contrast to the conclusion drawn by Park et al. [48], who suggest that their observed growth of the yield with $n_{a}$ results from a larger harmonic dipole amplitude for individual atoms in clusters due to their partial delocalization of the initial wave function.

Above $n_{a}=3 \times 10^{17} \mathrm{~cm}^{-3}(\langle N\rangle>1000$ atoms per cluster), a relatively slower growth trend (less than quadratic) is observed with increasing densities. Using our model with the calculated values of $g$, we can retrieve the $\mathrm{HH}$ yield from only gas monomers for both temperatures, namely, by setting $d_{\mathrm{cl}}^{\mathrm{HH} 21}(\langle N\rangle)$ to zero in Eq. 5.5 (blue dashed curves). This $\mathrm{HH}$ yield is plotted in both figures as blue dashed curves. It can be seen that the $\mathrm{HH}$ yield, for both temperatures, grows slower than quadratic with increasing density. It deviates from the red solid lines indicating a $\xi_{\mathrm{HH} 21} \propto n_{a}^{2}$ relation and approaches the blue dashed curves. This observation indicates that the harmonic generation from atoms in larger clusters becomes less efficient. Interestingly, such a slower growth trend is indeed also found by Park et al. [48] when the average cluster size produced in their supersonic argon jet becomes larger than 700 atoms per clusters. This relatively slow growth trend has earlier been explained as resulting from field screening of the laser that prevents further tunnel ionization of atoms inside the core of the larger clusters [141], and from stronger reabsorption of the XUV radiation inside the large clusters resulting in the formation of nanoplasmas [142].

\subsection{CONCLUSION}

We have investigated high-order harmonic generation in a supersonic argon gas jet. To identify the contributions of the generated high-order harmonics from both clusters and gas monomers, we measured the harmonic spectra over a broad range of the total atomic number densities (from $3 \times 10^{16} \mathrm{~cm}^{-3}$ to $3 \times 10^{18} \mathrm{~cm}^{-3}$ ) in the jet at two different reservoir temperatures ( $303 \mathrm{~K}$ and $363 \mathrm{~K}$ ). For the first time in the evaluation of the harmonic yield in such measurements, the detailed variation of the liquid mass fraction, $g$, versus pressure and temperature is taken into consideration. We determine this fraction, and find, consistently, low values of $g$ below $20 \%$, within our range of experimental parameters. Changing the temperature of the nozzle allows us to study the dependence of the $\mathrm{HH}$ yield on different values of $g$ and for the average cluster size, $\langle N\rangle$, while maintaining the value for the total atomic number density, $n_{a}$. Based on different values for $g$ at two different temperatures, a simple model is applied to interpret the measured, 
density-dependent harmonic spectra. We show that the single-atom response in clusters can is the same as the response of gas monomers, for clusters having an average size, $\langle N\rangle$, below $\sim 1000$ atoms per cluster. With increasing $\langle N\rangle$, HHG in clusters becomes less efficient. We also observe no change of the cut-off energy in the measured harmonic spectra, which indicates that the three-step model is still applicable for HHG in clusters, i.e., the tunnel ionized electrons collide with their parent ion. We conclude that using a supersonic gas jet to provide clusters as the nonlinear medium, does not promise a higher harmonic yield via an increased nonlinearity as compared to a gas jet of monomers. With this finding the use of cluster jets in high-order harmonic generation would rather be that an increased density contrast can be obtained for pursuing a higher yield via quasi-phase matching, such as by placing an array of obstacles. 


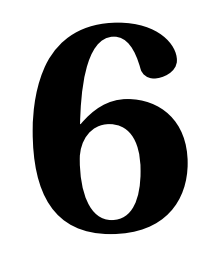

\section{A TEMPORAL QUASI-PHASE MATCHING MODEL FOR HIGH-ORDER HARMONIC GENERATION}

We present the first time-dependent model for quasi-phase matching (QPM) in high-order harmonic generation (HHG). Using a one-dimensional model, we analyze the time-dependent, ultrafast wave-vector balance to calculate the on-axis harmonic output versus time, from which we obtain the time-integrated, overall output. Considering, as an example, periodically patterned argon gas, as may be provided with a grid in a cluster jet, we calculate the harmonic output during different time intervals within the drive laser pulse duration. We find that identifying a suitable single spatial period is not straightforward due to the complex plasma dynamics in HHG. The maximum overall on-axis harmonic pulse energy is obtained when choosing the QPM period to phase match HHG at the leading edge of the drive laser pulse. 


\subsection{INTRODUCTION}

High-order harmonic generation (HHG) is an extremely nonlinear optical process that provides coherent radiation in the extreme ultraviolet spectral region on an ultrafast time scale. This radiation is of great interest for applications such as coherent diffractive imaging [126], pump-probe spectroscopy for studying dynamical electronic processes inside molecules [143] and attosecond science [144]. The range of generated wavelengths covered by the harmonics is usually limited. On the long wavelength side, the harmonic spectrum is limited by reabsorption in the generating medium and on the short wavelength side by lack of phase matching due to ionization. For example, the maximum harmonic order (the shortest wavelength) which can be phase matched in argon is the $35^{\text {th }}$ order $(\approx 24 \mathrm{~nm})$ for a $800 \mathrm{~nm}, 40$ fs drive laser pulse [88]. The laser intensities required for generating radiation of shorter wavelengths are so high that a significant fraction of the gaseous medium becomes ionized. As a result, the ultrafast change in the refractive index associated with the transient electron plasma renders phase matching techniques without effect.

A technique that is generally seen as suitable for overcoming this ionization limitation, for increasing the order of the efficiently generated harmonics, is so-called quasiphase matching (QPM) [88]. With the introduction of a spatially periodic structure with a suitable periodicity, an extra wave vector can be provided that compensates the wavevector mismatch. Various schemes to demonstrate or make use of QPM in HHG have been proposed and developed based on the idea that HHG is only enabled in the region where generated harmonics interfere constructively (in-phase region) and is suppressed in the region where generated harmonics interfere destructively (out-of-phase region). A train of tunable counter-propagating pulses in a hollow-core waveguide has been used to introduce a spatially periodic ionization pattern for the generation of photon energies of several hundred electron volts [41]. Other schemes include waveguides with a spatially periodic diameter [39], multimode beating in a capillary [40] and polarizationbeating in a birefringent waveguide [95, 96]. Recently, a small set of periodically spaced dual-gas jets, consisting of the generating gas (argon) and a passive gas (hydrogen), has shown to increase the conversion efficiency for the harmonic orders up to the $41^{\text {st }}$ order $(\approx 19 \mathrm{~nm})[45,93,94]$.

All these approaches are based on the assumption that, depending on the specific set of parameters used, there is a certain spatial period, $P_{\text {mod }}$, that provides phase matching. This assumption is much in analogy with nonlinear processes in periodically poled crystals where a particular conversion process with given input and output wavelengths requires a well-defined poling period. However, selecting an appropriate periodic structure for achieving QPM in HHG is actually rather problematic, since the wave-vector mismatch is strongly time dependent within the entire drive laser pulse. A fixed periodic structure may only be effective in achieving QPM during a short time interval within the drive laser pulse, such that the total output pulse energy for HHG may still remain low. To demonstrate QPM for HHG in experiments using the schemes mentioned above, the selection of a fixed periodic structure for the highest high-order harmonic $(\mathrm{HH})$ output is usually based on an unproven assumption, namely, that the quasi-phase matching period has to be chosen equal to the twice coherence length calculated at the peak intensity of the drive laser pulse. This is based on the argument that then the nonlinearly induced 
dipole moment of the individual atoms is the highest [27]. However, this assumption neglects any radiation that may be generated before and after the peak of the drive laser pulse. A direct experimental verification is difficult because, due to the ultrashort time scales, one is usually detecting or optimizing HHG via its pulse energy. Thus, for a proper discussion of optimizing QPM in HHG, it is required to provide a time-dependent modelling of the output followed by a calculation of the output pulse energy.

Here, we present the first time-dependent model for quasi-phase matching in highorder harmonic generation. Using a one-dimensional model, we include the full wavevector mismatch with its ultrafast time dependence to calculate the $\mathrm{HH}$ field vs. time. The pulse energy is then obtained by time integration. For definiteness and as a typical example we consider HHG in argon gas where a periodic density pattern may be provided, e.g., with a grid in a supersonic jet. We show that the maximum harmonic pulse energy is obtained when choosing the QPM period to phase match the harmonic generation at the leading edge of the drive laser pulse, rather than at the peak.

\subsection{ONE-DIMENSIONAL TIME-DEPENDENT MODEL FOR HHG}

As our basis for considering quasi-phase matching, we recall the well-known model of Constant et al. [91] and Kazamias et al. [145], which describes standard phase matching as achievable via pressure adjustment at weakly ionizing intensities. The instantaneous field for a specific harmonic order, $E_{q}(t)$, generated at the exit of the medium, where $q$ is the harmonic order, can be expressed as

$$
E_{q}(t) \propto \int_{0}^{L_{m e d}} \rho(z) \exp \left(-\frac{L_{m e d}-z}{2 L_{a b s}(z)}\right) d_{q}(z, t)[1-\eta(t)] \exp \left[i \phi_{q}(z, t)\right] d z .
$$

Here, $L_{m e d}$ is the length of the medium, $\omega$ is the angular frequency of the drive laser, $c$ and $\varepsilon_{0}$ are the speed of light and vacuum permittivity respectively, $\rho(z)$ is the initial atomic number density, $L_{a b s}(z)=1 /[\sigma \rho(z)]$ is the absorption length, where $\sigma$ is the ionization cross section [146], and $\phi_{q}(z, t)$ is the accumulated phase difference between the drive laser field and the generated harmonic field at the exit of the medium. The drive laser field is implicitly included in the induced atomic dipole moment amplitude, $d_{q}(z, t)$. Based on the full quantum theory developed by Lewenstein et al. [27], $d_{q}$ exhibits a strong dependence on time through its variation with the drive laser intensity $(I(z, t))$ and can be described approximately by the following power law [147].

$$
d_{q}(z, t)\left\{\begin{array}{ccc}
\propto\left(\frac{I(z, t)}{I_{t h r}(q)}\right)^{4.6} & \text { if } & I>I_{t h r}(q) \\
\approx 0 & \text { if } & I<I_{t h r}(q)
\end{array}\right.
$$

Here, $I_{t h r}(q)$ is the threshold laser intensity to be realized before there is a noticeable generation of the $q^{\text {th }}$ harmonic. The factor $1-\eta(t)$ in Eq. (6.2), where $\eta(t)$ is the ionization fraction, originates from ionization-induced ground state depletion, which indicates that the generated harmonic field scales with the number of neutral atoms. The ionization fraction, $\eta(t)$, can be calculated using the Ammosov-Delone-Krainov (ADK) model [78]. To give an example for the temporal variation of the ionization fraction and 


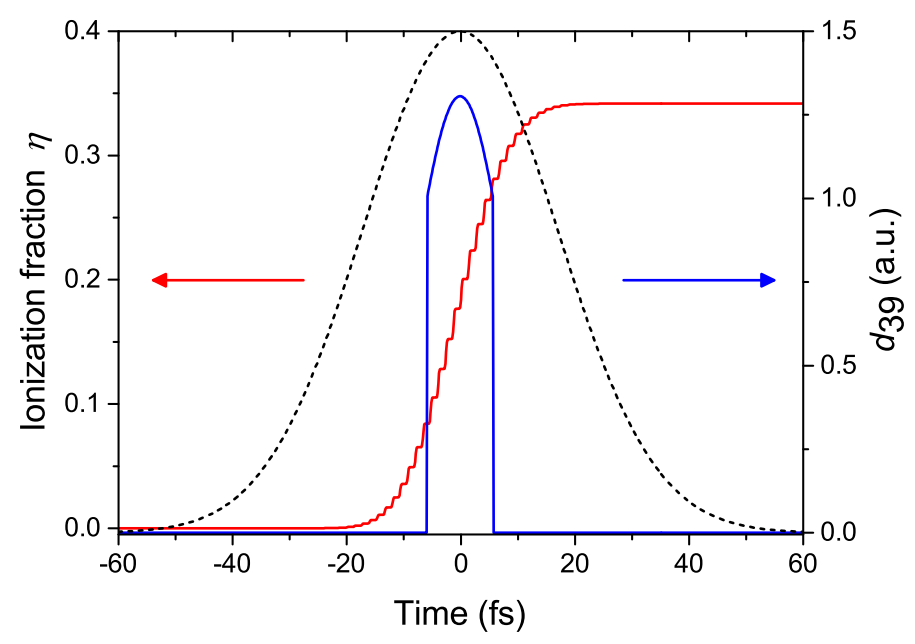

Figure 6.1: The ionization fraction, $\eta$, (red line) and the harmonic dipole amplitude, $d_{q}$, for the $39^{\text {th }}$ harmonic (blue line) calculated for argon and a drive laser pulse (Gaussian temporal shape) with a central wavelength at $800 \mathrm{~nm}$, a pulse duration of $\tau=40$ fs $(F W H M)$ and a peak intensity of $I_{0}=2.5 \times 10^{14} \mathrm{~W} / \mathrm{cm}^{2}$. The drive laser pulse (black dashed line) is plotted as a reference.

individual harmonic dipole amplitude, Fig. 6.1 shows a calculation of the ionization fraction and the $39^{\text {th }}$ harmonic dipole amplitude, $d_{39}$ of argon for a drive laser pulse with a peak intensity of $I_{0}=2.5 \times 10^{14} \mathrm{~W} / \mathrm{cm}^{2}$. The laser pulse has a Gaussian shape with a pulse duration of $\tau=40 \mathrm{fs}$ (FWHM) and a central laser wavelength of $800 \mathrm{~nm}$. As can be seen from the red curve, the ionization fraction reaches about $18 \%$ at the peak of the laser intensity and raises further to about $34 \%$ towards the end of the laser pulse. It can further be noticed that the ionization fraction grows with each optical cycle resembling a stepwise increase (here 15 steps in total). The dipole amplitude exhibits a strong dependence on the drive laser intensity through the threshold and power law in Eq. (6.2).

The term $\phi_{q}(z, t)$ in Eq. (6.1), is directly related to the wave-vector mismatch, $\Delta k(z, t)$, between the drive laser and the generated harmonics. The accumulated phase difference after a propagation length of $L_{m e d}$ can be described as

$$
\phi_{q}\left(L_{\text {med }}, t\right)=\int_{0}^{L_{\text {med }}} \Delta k(z, t) d z .
$$

Here, as will be detailed below, the mismatch, $\Delta k(z, t)$, originates from the dispersion due to neutral gas atoms $\left(\Delta k_{\text {atom }}\right)$, electrons in the plasma that is generated via ionization $\left(\Delta k_{\text {plasma }}\right)$, the Gouy phase shift, which depends on the focusing geometry of the drive laser $\left(\Delta k_{g e o}\right)$, and the intrinsic harmonic dipole phase $\left(\Delta k_{\text {dipole }}\right)$. An idealized description of all these contributions, i.e., neglecting any time-dependence, has been introduced in Chapter 2. However, several parameters in the named wave-vector contributions are not only space-dependent but also strongly time-dependent. Analyzing the 
consequence of these dynamic effects for quasi-phase matching is the purpose of this chapter. Taking the time-dependence into account, Eq. (2.51) turns into the following expression

$$
\Delta k(z, t)=\Delta k_{\text {atom }}(z, t)+\Delta k_{\text {plasma }}(z, t)+\Delta k_{\text {geo }}(z)+\Delta k_{\text {dipole }}(z, t)
$$

The contribution of the neutral atomic dispersion to the total wave-vector mismatch is positive and can be expressed by

$$
\Delta k_{\text {atom }}(z, t)=q[1-\eta(z, t)] \frac{2 \pi P(z)}{\lambda} \Delta n .
$$

Here, $P(z)$ is the pressure of the gas in mbar, $\lambda$ is the wavelength of the drive laser and $\Delta n$ is the difference between the indices of refraction of the gas per mbar at the drive laser wavelength, $\lambda$, and the high harmonic wavelength, $\lambda / q$. The dependence on $\eta(z, t)$ shows that the mentioned atomic dispersion decreases during the drive laser pulse.

The contribution due to the free electrons in the plasma, on the contrary, is negative and becomes

$$
\Delta k_{\text {plasma }}(z, t)=-\eta(z, t) P(z) N_{a} r_{e} \frac{q^{2}-1}{q} \lambda,
$$

where $N_{a}$ is the initial number density of atoms per mbar, and $r_{e}$ is the classical radius of the electron. This contribution via $\eta(z, t)$ grows in magnitude vs. time.

The wave-vector mismatch that arises from the Gouy phase shift, resulting from the focusing of the drive laser beam, only varies in space. It also has a negative sign and can be expressed by

$$
\Delta k_{\text {geo }}(z)=-\frac{1}{z_{R}+\frac{\left(z-z_{0}\right)^{2}}{z_{R}}}(q-1),
$$

where $z_{0}$ is the position of the focus and $z_{R}$ is the Rayleigh range.

Finally, the contribution of the intrinsic harmonic dipole phase originates from the intensity dependence of the trajectories that the transiently ionized electrons follow before recombination and harmonic generation, which can be described as [85]

$$
\Delta k_{\text {dipole }}(z, t)=-\alpha_{q} \frac{\partial I(z, t)}{\partial z}=\frac{2\left(z-z_{0}\right)}{z_{R}^{2}\left[1+\left(\frac{\left(z-z_{0}\right)}{z_{R}}\right)^{2}\right]^{2}} \alpha_{q} I_{0},
$$

where $\alpha_{q}$ is a coefficient related to the trajectories which the electrons take. This expression can be chosen to be either positive or negative depending on the position of the focus with regard to the generating medium. Placing the medium in front of (behind) the focus, where $z<z_{0}\left(z>z_{0}\right)$, results in a negative (positive) term. When the focus is placed in the center of the medium $\left(z=z_{0}\right)$, such as to maximize the drive laser intensity, the value of $\Delta k_{\text {dipole }}(z, t)$ becomes equal to zero.

The terms described so far can now be used to calculate the spatial and temporal evolution of the harmonic field, as expressed in Eq. (6.1). The instantaneous harmonic intensity of the $q^{\text {th }}$ harmonic at the exit of the medium is obtained from $I_{q}(t) \propto$ 
$\frac{1}{2} c \varepsilon_{0}\left|E_{q}(t)\right|^{2}$. The on-axis harmonic pulse energy for the $q^{\text {th }}$ harmonic, $F_{q}$, can be obtained by temporal integration over the entire pulse and multiplying with an interaction area, $S(z)$,

$$
F_{q}=S(z) \int_{-\infty}^{\infty} I_{q}(t) d t
$$

The model has been widely and successfully applied to analyze the measurements of HHG under various experimental conditions such as a guided-wave geometry [88, 102], or loosely focusing geometry [33, 91, 148, 149]. In the next section, we will use the model in its simplest form, namely, for the case of a homogeneous medium (argon) with a loosely focused drive laser beam. In detail, we will present the consequence of the time dependence in the wave-vector mismatch.

\subsection{TIME-DEPENDENCE OF PHASE MATCHING IN A HOMOGE- NEOUS MEDIUM}

In the case of a loose focusing geometry for HHG, the Rayleigh range is chosen to be long, typically in the order of tens of millimeters such that it becomes much longer than the length of the medium $\left(z_{R} \gg L_{m e d}\right)$. Although loose focusing reduces the drive laser intensity, the advantage is that a large interaction volume can be provided for maximizing the total harmonic pulse energy, and usually a relatively low gas pressure is required to maintain phase matching [33, 149-151]. In this geometry, the drive laser intensity stays approximately constant along the entire generating medium. Therefore, the harmonic dipole amplitude, $d_{q}(z, t)$, the interaction area, $S(z)$, and also the ionization fraction, $\eta(z, t)$, become space independent. Due to the long Rayleigh range, the geometryinduced wave-vector contribution can be simplified as $\Delta k_{g e o}=-(q-1) / z_{R}$ and becomes independent of $z$. In addition, the harmonic dipole phase contribution, $\Delta k_{\text {dipole }}(z, t)$, becomes much smaller than the other terms [145] and can be neglected. As a result, the total wave-vector mismatch can be regarded as a space-independent parameter, $\Delta k(t)$, such that the phase term, $\phi_{q}(z, t)$, in Eq. (6.1) can be simply represented as the product of $\Delta k(t)$ and $z$ :

$$
\phi_{q}(z, t)=\Delta k(t) \cdot z=\frac{\pi z}{L_{c o h}(t)} .
$$

Here, the time-dependent coherence length, $L_{c o h}(t)=\pi / \Delta k(t)$, is introduced for characterizing the wave-vector mismatch in comparison to other length parameters, specifically the length of the medium. The physical meaning of $L_{c o h}$ is that the $q^{\text {th }}$ harmonic radiation generated at the end of this length is shifted out of phase with harmonic radiation generated from the beginning of the length, which leads to back conversion in futher propagation, often also termed as destructive inteference. By implementing a time-dependent coherence length, Eq. (6.1) can be integrated analytically and the instantaneous HH intensity reads as [91, 147]

$$
\begin{aligned}
I_{q}(t) \propto \rho^{2}[1-\eta(t)]^{2} d_{q}(t)^{2} \frac{4 L_{a b s}^{2}}{1+4 \pi^{2}\left[L_{a b s}^{2} / L_{c o h}(t)^{2}\right]} \\
\times\left[1+\exp \left(-\frac{L_{m e d}}{L_{a b s}}\right)-2 \cos \left(\pi \frac{L_{m e d}}{L_{c o h}(t)}\right) \exp \left(-\frac{L_{m e d}}{2 L_{a b s}}\right)\right] .
\end{aligned}
$$


In this expression, also reabsorption of harmonic radiation is taken into account via an absorption length, $L_{a b s}$. From Eq. (6.11) it can be seen that, ideally, the maximum harmonic output can be obtained when perfect phase matching is achieved, namely, $\Delta k(t)=0$ (equivalent to an infinitely long coherence length) during the entire drive laser pulse. In particular, when absorption is neglected (infinitely long absorption length) the harmonic intensity would grow proportionally to the square of the medium length. For the case that phase matching is not fulfilled $(\Delta k \neq 0)$ and can be described by a temporally constant and finite coherence length, i.e., when the ionization fraction does not change vs. time, and when additionally absorption cannot be neglected, a coarse criterion, $L_{c o h}>L_{\text {med }}>3 L_{a b s}$, has been proposed [91, 149, 150]. If this criterion is fulfilled, the harmonics are efficiently generated and limited only by absorption. Quantitatively, for instance, when $L_{c o h}>5 L_{a b s}$ and $L_{m e d}>3 L_{a b s}$, the harmonic intensity reaches more than half of the maximum intensity of the ideal case $\left(L_{c o h} \rightarrow \infty, L_{a b s} \rightarrow \infty\right)$. However, when generating high-order harmonics with an increased intensity and appreciable gas density, the named criterion becomes inapplicable because the coherence length is not a constant parameter. In that case, the coherence length varies strongly on an ultrafast time scale due to a dramatic change of the ionization fraction of the medium during the drive pulse. Therefore, phase matching is only achieved in a very short time interval during the drive pulse. As a result, it remains questionable to what fraction and at what instance during the drive laser pulse the criterion should be fulfilled for a maximum harmonic output pulse energy. A further complication is that the harmonic dipole amplitude, $d_{q}(t)$, is also a time-dependent parameter that will influence the HH output. For instance, even if the above length criterion is fulfilled for a certain time interval, the output pulse energy may still be far below optimum if $d_{q}$ is small during that interval.

In view of these effects, it is important to analyze the time dependence of the coherence length as well as of the harmonic dipole amplitude over the entire drive laser pulse. Here, for definiteness, we take two harmonic orders for HHG in argon as an example, namely, a moderate harmonic order, the $21^{\text {st }}$ harmonic, and a relatively high harmonic order, the $39^{\text {th }}$ harmonic. Fig. 6.2 shows the time-dependent coherence lengths (black curves) for the $21^{\text {st }}$ harmonic (a) and the $39^{\text {th }}$ harmonic (b) calculated for a HHG experiment in a typical loose focusing configuration, assuming a drive laser peak intensity of $I_{0}=2.5 \times 10^{14} \mathrm{~W} / \mathrm{cm}^{2}$, a Rayleigh range of $z_{R}=18 \mathrm{~mm}$ as achieved by a f/150 focusing lens, a medium length of $L_{\text {med }}=5 \mathrm{~mm}$ and an argon gas pressure of $30 \mathrm{mbar}$. The laser pulse (also shown in Fig. 6.2) is again taken to have a Gaussian shape (blue curve) with a pulse duration of $\tau=40 \mathrm{fs}$ (FWHM). The lengths of the medium (dashed blue lines) and the calculated absorption lengths (dashed pink line) for both harmonics are also plotted for comparison. Furthermore, the green areas in Fig. 6.2 (a) and (b) indicate the time intervals of sufficiently high intensity, in which, potentially, each respective harmonic can be generated $\left(I>I_{t h r}\right)$. Finally, the time-dependent coherence lengths are plotted as solid black curves.

It can be clearly seen that for both harmonics, the coherence length shows an ultrafast time dependence. Specifically, starting from a certain mismatch, there is first a rapid increase of $L_{c o h}$ in some part of the leading edge of the drive laser pulse. Initially, the dispersion of free electrons due to the negligible ionization fraction is relatively small, and the other terms in Eq. (6.4) dominate. However, with the increase of the drive laser in- 

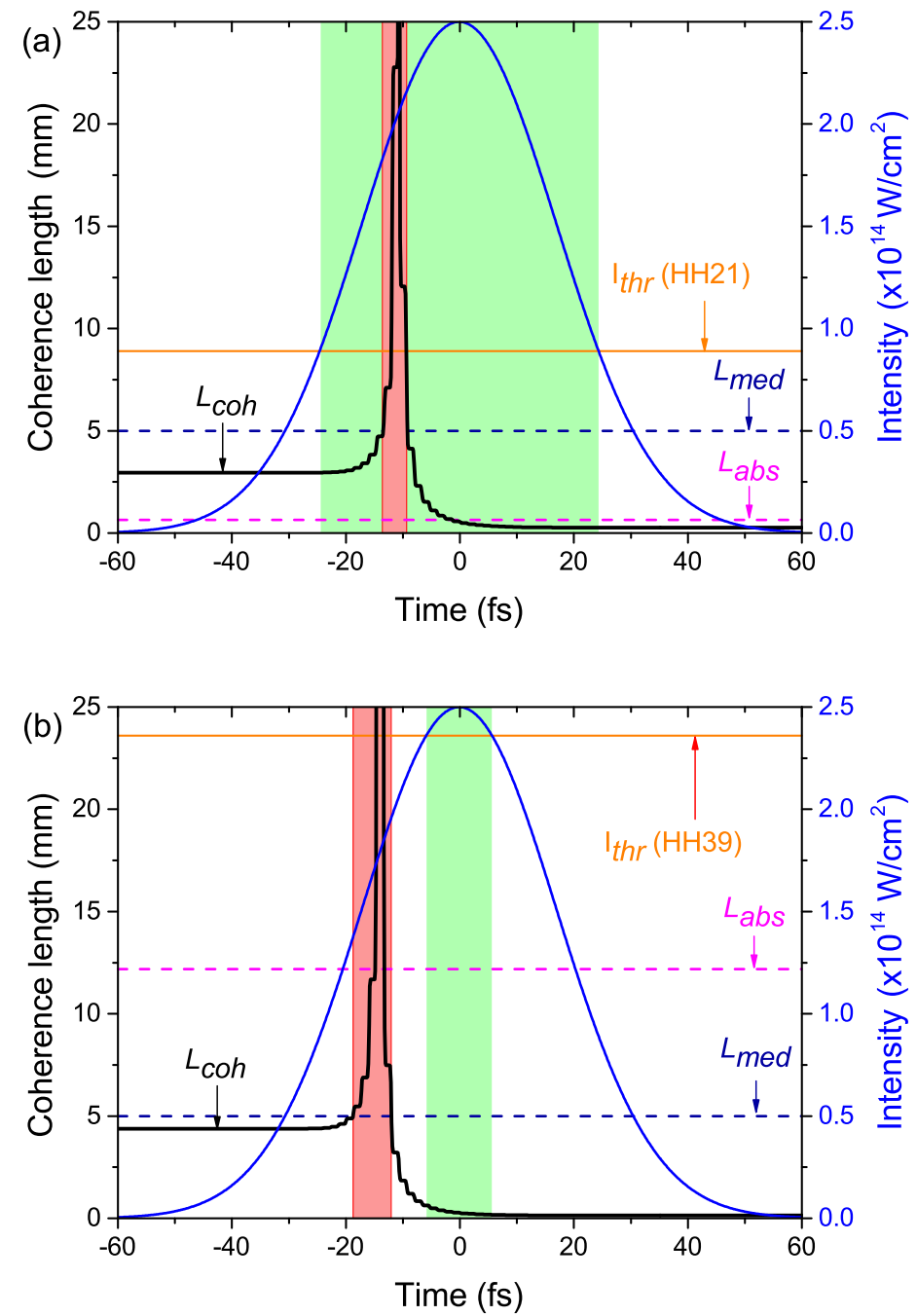

Figure 6.2: Calculated coherence lengths (black curves) for the $21^{\text {st }}$ harmonic (a) and the $39^{\text {th }}$ harmonic (b) as a function of time. Assumed is harmonic generation in $30 \mathrm{mbar}$ of argon in a $5 \mathrm{~mm}$ long medium ( $L_{\text {med }}=5 \mathrm{~mm}$, dashed blue lines) with a Gaussian-shaped laser pulse of $2.5 \times 10^{14} \mathrm{~W} / \mathrm{cm}^{2}$ peak intensity (blue curves). The absorption lengths (dashed pink lines) and the minimum required laser intensity (solid orange lines) are calculated for both harmonics and plotted for comparison. The green areas show within which time interval there is a sufficiently high drive laser intensity for HHG $\left(I>I_{t h r}\right)$. The red areas indicate the time interval during which phase-matching condition is approximately fulfilled, i.e., the harmonic output from the entire medium adds constructively $\left(L_{c o h}>L_{m e d}\right)$, if the respective harmonic is generated.

tensity, the ionization fraction rapidly increases further. Consequently, the dispersion in the plasma due to the accumulation of free electrons overwhelms the other terms in 
Eq. (6.4), resulting in a quick drop of the coherence length. When the ionization increases rapidly, there is an extremely short interval (a few femtoseconds long) where the dispersion is zero (phase matching). For the $21^{\text {st }}$ harmonic (Fig. 6.2 (a)), the time interval of phase matched generation is indicated by the red area. This interval is found in the leading edge of the drive laser pulse (where the criterion $L_{c o h}>L_{\text {med }}(5 \mathrm{~mm})>$ $3 L_{a b s}(0.7 \mathrm{~mm})$, is fulfilled). It can be seen that this interval overlaps with the time interval where the drive laser intensity is sufficiently high for generating the $21^{\text {st }}$ harmonic, which is indicated as the green area. The overlap indicates that the $21^{\text {st }}$ harmonic can be efficiently generated at least within the short overlapping time interval. For the $39^{\text {th }}$ harmonic (Fig. 6.2 (b)), the coherence length reaches a very large value and also becomes longer than the length of the medium in a short time interval at the leading edge of the pulse (red area). However, in contrast to the $21^{\text {st }}$ harmonic, this interval is located outside the time interval in which the $39^{\text {th }}$ harmonic can be generated (green area, where $I>I_{t h r}$ (HH39)). Thus, the green (phase matching) and red (generation) intervals do not overlap, which indicates that the ionization fraction induced by the minimum required drive laser intensity for generating the $39^{\text {th }}$ harmonic is too high for phase matching. The wave-vector mismatch, $\Delta k_{\text {plasma }}$, due to the large amount of free electrons dominates over the other wave-vector contributions. In this example, for argon, this occurs for harmonic orders higher than $q=35$. Consequently, the $39^{\text {th }}$ harmonic will not be efficiently generated at any moment throughout the entire drive laser pulse. To illustrate the differ-

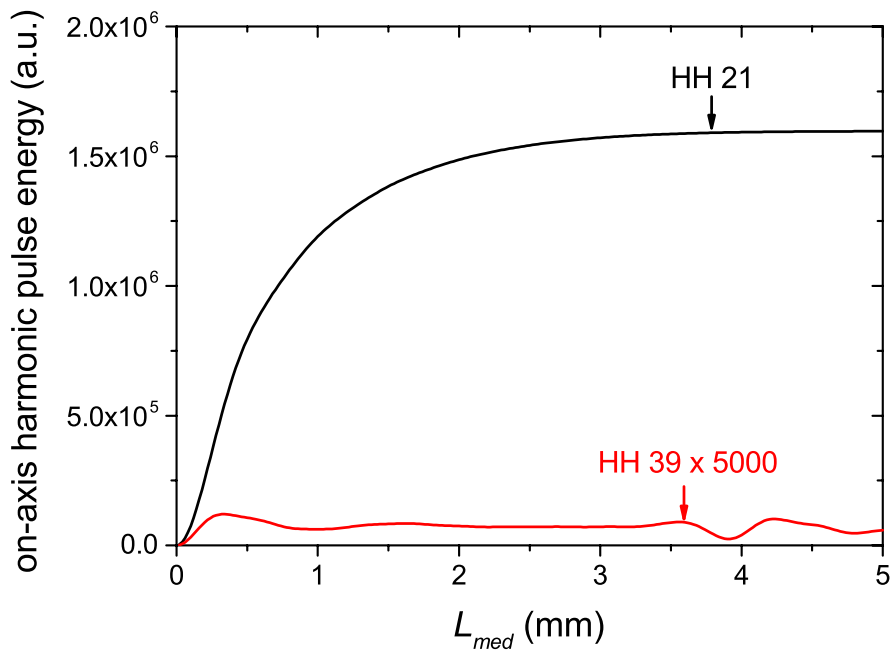

Figure 6.3: Calculated HH pulse energy for the $21^{\text {st }}$ (black) and the $39^{\text {th }}$ harmonic (red) as a function of the medium length. For $L_{m e d}>1 \mathrm{~mm}$, the $39^{\text {th }}$ harmonic output is about four to five orders of magnitude weaker.

ence between the two considered harmonics also quantitatively, we have calculated the on-axis harmonic output pulse energy, $F_{q}$, as a function of interaction length using Eqs. (6.9) and (6.11), which is plotted in Fig. 6.3. It can be seen that the output energy of the $21^{\text {st }}$ harmonic (black curve) grows strongly within the first few $\mathrm{mm}$ of the medium and saturates after roughly 3 to $4 \mathrm{~mm}$. This observation shows that the harmonic is efficiently 
generated and only limited by reabsorption. On the other hand, the output pulse energy of the $39^{\text {th }}$ harmonic (red curve) does not show any obvious growth with the length of the medium and is (beyond $L_{\text {med }}=1 \mathrm{~mm}$ ) approximately four to five orders of magnitude weaker. A few oscillations can be seen along the length of the medium, which is due to varying levels of destructive interference.

From a series of additional calculations, we conclude that for moderate harmonic orders up to $q=35$ in this example, the length criterion for efficient output $\left(L_{c o h}>L_{\text {med }}>\right.$ $3 L_{a b s}$ ) can be fulfilled over at least some certain short time interval falling within the interval that the drive laser intensity is above the generation threshold. This allows for harmonic generation that is limited only by absorption. However, for harmonics of $q>35$ (such as in the example above, $q=39$ ), the criterion can never be fulfilled at any instance during the drive laser pulse, which leads to a generation efficiency that is decreased by many orders of magnitude.

A solution to overcome such low efficiencies for higher orders is to implement QPM techniques into the HHG configuration. In Section 6.4, we present a specific maximally simplified QPM scheme based on providing a periodic density modulation.

\subsection{HHG IN A PERIODIC DENSITY MODULATED MEDIUM}

The basic idea of quasi-phase matching (QPM), also for HHG, is to introduce some periodic modulation of the local strength or phase of the generation process throughout the medium. To counteract the destructive interference between locally generated harmonic fields that are generated in subsequent coherence lengths, a straightforward approach would be to suppress the harmonic generation within every other coherence length. By repeating the suppression periodically in the spatial domain, such modulation is in the Fourier domain equivalent to providing an additional wave-vector contribution, $K_{Q P M}$, that compensates the remaining wave-vector mismatch $(\Delta k,(z, t)$ in Eq. (6.4)). As was shown above for harmonics in argon with an order beyond $q=35$, the mismatch that needs to be compensated originates predominately from the plasma dispersion. The total wave-vector mismatch $\Delta k^{\prime},(z, t)$, for HHG including QPM can be expressed as $\Delta k^{\prime},(z, t)=\Delta k,(z, t)-K_{Q P M}$. The latter expression looks simple. However, two points have to be noted. While the mismatch $\Delta k(z, t)$ changes on an ultrafast time scale, the additional wave-vector, $K_{Q P M}$, essentially has a temporally constant value in the case of using a periodic density modulated medium because it is difficult to change any gas density distribution on an ultrashort time scale as well. Consequently, quasiphase matching will only be fulfilled transiently again, for a certain short time interval within the drive laser pulse, as discussed above. Moreover, because the density of the medium is spatially modulated, the mismatch attains an additional, periodic space dependence.

Because experimentally, it is the simplest way of spatial-periodically suppressing destructive contributions in HHG, we consider here the introduction of a periodic modulation of the gas atomic density along the propagation axis, as is depicted in Fig. 6.4. Such a density distribution might be achieved, e.g., by placing a periodic grid in a supersonic gas jet. To express the density modulation quantitatively, the length of the medium can be written as: $L_{\text {med }}=N P_{\text {mod }}=N\left(L_{h i g h}+L_{l o w}\right)$, where $N$ is the number of modulation periods, $P_{m o d}$ is the modulation period, and $L_{h i g h}$ and $L_{l o w}$ are the lengths of the high- 
(a)

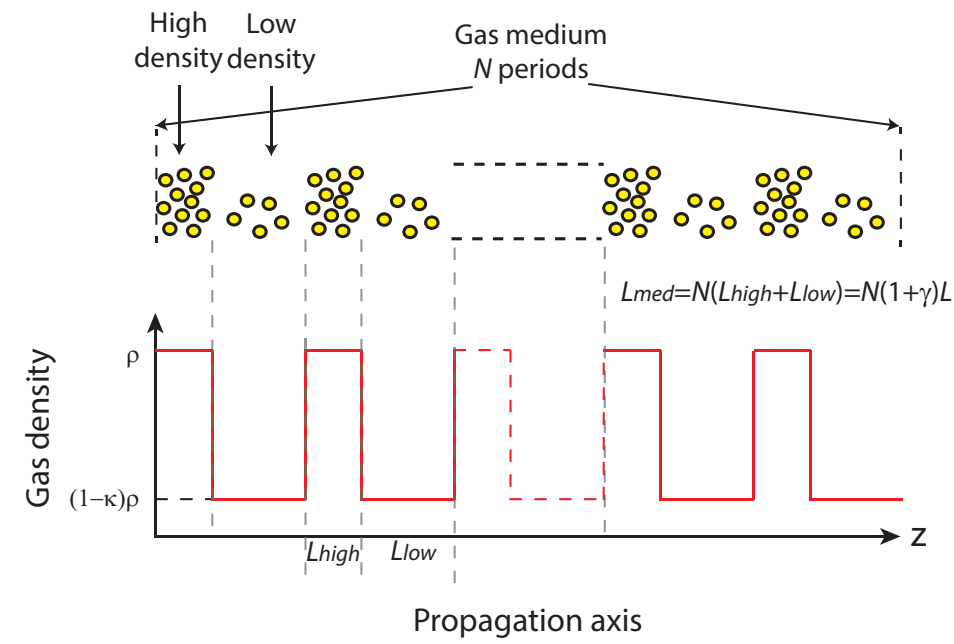

(b)

Figure 6.4: (a) Schematic of a density modulated medium consisting of alternating regions with high and low gas density. (b) The corresponding gas density profile can be described via a maximum gas density, $\rho$, and a modulation depth, $\kappa$. The lengths of the high-density and low-density regions are $L_{\text {high }}$ and $L_{l o w}$, respectively.

density and low-density regions, respectively. The corresponding modulated gas density profile along the propagation axis of the drive laser, $z$, is shown in Fig. 6.4 (b) using the simplifying assumption of homogeneous densities within $L_{h i g h}$ and $L_{l o w}$. We define the modulation depth of the gas density by a factor $\kappa$. When $\kappa=1$, the gas density is fully modulated, while if $\kappa=0$, there is no modulation imposed on the gas. To obtain the instantaneous on-axis harmonic field, $E_{q}(t)$, in a density modulated medium with a loose focusing geometry, one has to take into account that several additional parameters become space dependent in the integral in Eq. (6.1), specifically, the gas density, $\rho(z)$, the phase term, $\phi_{q}(z, t)$ and the absorption length, $L_{a b s}(z)$. To evaluate Eq. (6.1), we rewrite the integral as a sum over the field contributions from the high-density and low-density regions as

$$
E_{q}(t)=\sum_{n=1}^{N}\left[E_{q, n}^{h i g h}(t)+E_{q, n}^{l o w}(t)\right]
$$

where $E_{q, n}^{\text {high }}(t)$ and $E_{q, n}^{\text {low }}(t)$ are the $q^{\text {th }}$ harmonic field generated in the high-density and low-density regions of the $n^{\text {th }}$ modulation period, respectively. The phase term, $\phi_{q, n}(z, t)$, can be calculated from the wave-vector mismatch in the regions of different densities as follows

$$
\phi_{q, n}(z, t)=\left\{\begin{array}{r}
(n-1)\left[\Delta k_{h i g h}(t) L_{h i g h}+\Delta k_{l o w}(t) L_{l o w}\right] \\
+\Delta k_{h i g h}(t) \cdot z \quad \text { in any high-density region, } \\
(n-1)\left[\Delta k_{h i g h}(t) L_{h i g h}+\Delta k_{l o w}(t) L_{l o w}\right] \\
+\Delta k_{h i g h}(t) L_{h i g h}+\Delta k_{\text {low }}(t) \cdot z \quad \text { in any low-density region. }
\end{array}\right.
$$

Here, $\Delta k_{\text {high }}(t)$ and $\Delta k_{\text {low }}(t)$ are the wave-vector mismatch in the high-density and low- 
density regions, respectively. Similarly, the coherence length in the high-density and low-density regions can be written as: $L_{c o h}^{\text {high }}(t)=\pi / \Delta k_{h i g h}(t)$ and $L_{c o h}^{l o w}(t)=\pi / \Delta k_{\text {low }}(t)$.

Next, in order to investigate the influence of QPM on efficient harmonic generation rather than on limitations due to reabsorption, we restrict ourselves to the situation that the length of the medium is shorter than the absorption length. As was described in Section 6.3, this can be achieved with a sufficiently low gas pressure in a loose focusing geometry. Indeed, the absorption lengths for the higher-order harmonics ( $q=35$ to 49 ) are typically much longer than the length of the medium $(5 \mathrm{~mm})$ over a broad tuning range of the gas pressure (density). For example, the absorption length for the $39^{\text {th }}$ harmonic at a pressure of $30 \mathrm{mbar}$ is about $12 \mathrm{~mm}$ long, assuming no modulation $(\kappa=0)$ is achieved. Finally, using the equation for the sum of a geometric series, Eq. (6.12), the expression for the instantaneous on-axis harmonic field can be simplified, and reads

$$
E_{q}(t)=\xi_{q}^{\text {high }}(t)+\xi_{q}^{l o w}(t)
$$

where $\xi_{q}^{h i g h}(t)$ is the sum of generated harmonic field contributions from only the highdensity regions:

$$
\xi_{q}^{h i g h}(t) \propto \frac{\rho d_{q}(t)}{i \pi / L_{c o h}^{h i g h}(t)}\left[\exp \left(i \frac{\pi L_{h i g h}}{L_{c o h}^{h i g h}(t)}\right)-1\right] \frac{1-\exp \left[i N \pi\left(\frac{L_{h i g h}}{L_{c o h}^{h i g h}(t)}+\frac{L_{l o w}}{L_{c o h}^{\text {low }}(t)}\right)\right]}{1-\exp \left[i \pi\left(\frac{L_{h i g h}}{L_{c o h}^{\text {high }}(t)}+\frac{L_{\text {low }}}{L_{c o h}^{\text {low }}(t)}\right)\right]},
$$

and where $\xi_{q}^{\text {low }}(t)$ is the generated harmonic field contribution from only the low-density regions:

$$
\begin{aligned}
\xi_{q}^{\text {low }}(t) \propto \frac{(1-\kappa) \rho d_{q}(t)}{i \pi / L_{c o h}^{l o w}(t)}\left[\exp \left(i \frac{\pi L_{l o w}}{L_{c o h}^{\text {low }}(t)}\right)-1\right] & \exp \left[i \frac{\pi L_{h i g h}}{L_{c o h}^{\text {high }}(t)}\right] \\
& \times \frac{1-\exp \left[i N \pi\left(\frac{L_{\text {high }}}{L_{c o h}^{\text {high }}(t)}+\frac{L_{\text {low }}}{L_{c o h}^{\text {low }}(t)}\right)\right]}{1-\exp \left[i \pi\left(\frac{L_{h i g h}}{L_{c o h}^{\text {high }}(t)}+\frac{L_{l o w}}{L_{c o h}^{\text {low }}(t)}\right)\right]} .
\end{aligned}
$$

With these expressions, the instantaneous on-axis intensity, $I_{q}(t)$, for the $q^{\text {th }}$ harmonic from a density modulated medium becomes

$$
I_{q}(t) \propto \frac{1}{2} c \varepsilon_{0}\left|\xi_{q}^{h i g h}(t)+\xi_{q}^{l o w}(t)\right|^{2} .
$$

The corresponding on-axis HH pulse energy of the $q^{\text {th }}$ harmonic, $F_{q}$, can be obtained again by integration over the entire pulse (Eq. (6.9)).

From Eqs. (6.15) and (6.16), it can be seen again that the coherence length (in both the high-density and low-density regions, $L_{c o h}^{\text {high }}$ and $L_{c o h}^{l o w}$ ) varies strongly on an ultrafast time scale via the ionization fraction. Therefore, similar to standard phase matching discussed above for lower-order harmonics, QPM can only be transiently achieved in a short time interval within the drive laser pulse. However, by choosing the value of the modulation period, this time interval can be shifted to a proper instance, such that the $\mathrm{HH}$ pulse energy is maximized. 


\subsection{Results and Discussion OF HHG With QPM}

In this section, we reconsider the generation of the $39^{\text {th }}$ harmonic assuming the same loose focusing parameters given in Section 6.3 (a drive laser peak intensity $I_{0}=2.5 \times 10^{14}$ $\mathrm{W} / \mathrm{cm}^{2}$, a Rayleigh range $z_{R}=18 \mathrm{~mm}$ achieved by a f/150 focusing lens, $L_{\text {med }}=5 \mathrm{~mm}$ ) and the same argon gas pressure (30 mbar). Based on the QPM model described in Section 6.4, we calculate the instantaneous on-axis HH intensity, $I_{39}(t)$, and the harmonic pulse energy, $F_{39}$, by applying different modulation periods, $P_{\text {mod }}$, and modulation depths, $\kappa$. We note that, to re-phase the harmonic wave with the fundamental wave after each coherence length in the high-density regions, the low-density regions have to provide a phase shift of $\pi$ between the generated harmonic wave and the fundamental wave. This means that also the low-density regions have to be as long as a coherence length. As a result, the desired modulation period should be equal to the sum of the coherence length calculated in both high-density and low-density regions, $P_{\text {mod }}=L_{c o h}^{\text {high }}+L_{c o h}^{\text {low }}$.

In order to choose an appropriate modulation period, $P_{m o d}$, the time-dependent coherence length for both high-density and low-density regions needs to be calculated. Fig. 6.5 shows the calculated coherence lengths for the $39^{\text {th }}$ harmonic (coloured curves)

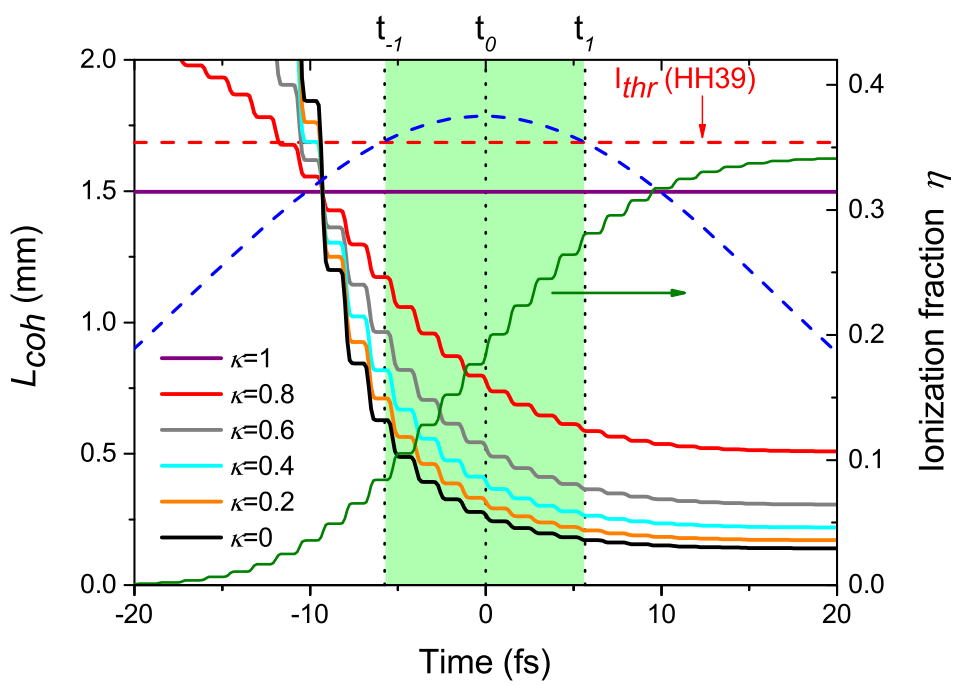

Figure 6.5: Calculated coherence lengths for the $39^{\text {th }}$ harmonic as a function of time for various modulation depths, $\kappa$ (1 (purple), 0.8 (red), 0.6 (grey), 0.4 (cyan), 0.2 (orange) and 0 (black)). The ionization fraction (dark green curve) and the temporal profile of the drive laser intensity (blue dashed line) are plotted for reference. The red dashed line is the threshold intensity for the $39^{\text {th }}$ harmonic generation and the green area indicates the time interval (from $t_{-1}$ to $t_{1}$ ) during which the generation of the $39^{\text {th }}$ harmonic is possible. The generating medium is $5 \mathrm{~mm}$ long and the gas pressure is set at $30 \mathrm{mbar}$ in the high-density region. The drive laser pulse has a peak intensity of $2.5 \times 10^{14} \mathrm{~W} / \mathrm{cm}^{2}$.

in the low-density regions as a function of time. The modulation depth, $\kappa$, is varied from 0 to 1 in steps of 0.2 during the drive laser pulse (the blue dashed curve). The coherence 
length for the high-density regions is depicted as the black curve $(\kappa=0)$. The green area indicates the time interval where the drive laser intensity is above the intensity threshold (red dashed line) for generating the $39^{\text {th }}$ harmonic. In Fig. 6.5, three characteristic times are introduced and shown along the top axis, where $t_{-1}$ represents the on-set of the generation of the $39^{\text {th }}$ harmonic, $t_{0}$ is the moment at which the drive laser pulse reaches its peak intensity, and $t_{1}$ indicates the cease of the generation of the $39^{\text {th }}$ harmonic. For full modulation ( $\kappa=1$, purple line), the coherence length for the low-density region becomes independent of time due to the absence of any gas that can be ionized. In this limiting case, the coherence length is only given by the wave-vector mismatch, $\Delta k_{g e o}$, due to the Gouy phase shift, which is set by the focusing geometry. The coherence lengths for the modulation depths with $\kappa<1$ are strongly time-dependent and drop rapidly during the pulse due to the increasing ionization fraction (dark green curve), specifically within the time interval where the $39^{\text {th }}$ harmonic is generated (green area). The information in the figure can be used as follows: if the harmonic output is to be maximized at a particular instance, one can read the required QPM period from the figure. For instance, if the output is to be maximized in a short time interval at the peak of the drive laser pulse, the crossing of the coherence lengths with the dashed vertical line at that time $\left(t_{0}\right)$ gives the length of both the high-density and low-density regions that yields QPM at that time. For instance, for $\kappa=0.8$, one obtains $L_{c o h}^{\text {low }}=0.79 \mathrm{~mm}$ (red curve) and $L_{c o h}^{\text {high }}=0.27 \mathrm{~mm}$ (black curve), resulting in a modulation period of $P_{\text {mod }}=L_{c o h}^{\text {high }}+L_{c o h}^{l o w}=1.06 \mathrm{~mm}$.

Now that we have illustrated how to choose the modulation period that maximizes $I_{q}$ in Eq. (6.17) at the peak of the drive laser pulse, $t_{0}$, we present in Fig. 6.6 a calculation of how the $\mathrm{HH}$ intensity $\left(I_{39}\right)$ generated at the drive laser peak grows vs. the propagation coordinate through the medium, $z$. For comparison, we also plot the spatial growth of $I_{39}$ generated at other characteristic instances of the drive laser pulse in the leading edge $\left(t_{-1}=-5.7 \mathrm{fs}\right)$ and the trailing edge $\left(t_{1}=5.7 \mathrm{fs}\right)$. With the modulation period, $P_{\text {mod }}$, set to provide QPM at $t_{0}$, we also display the influence of the modulation contrast by choosing three different modulation depths. For reference, we also plot the growth of $I_{39}$ generated at the peak of the drive laser pulse in a homogeneous medium, i.e., without density modulation. From Fig. 6.6 it can be clearly seen that, in the absence of density modulation, the harmonic intensity generated at $t_{0}=0$ fs does not grow vs. the interaction length but only oscillates periodically between 0 and some smaller value. This oscillation, indicating periodic generation and back conversion, which is caused by a rather short coherence length (in this case about $270 \mu \mathrm{m}$ ). However, when the medium is periodically modulated, the harmonic intensity of the $39^{\text {th }}$ harmonic generated at $t_{0}=0$ fs (black curves) shows a clear growth of the intensity with the medium length with all shown examples of the modulation depth. Specifically, the intensity generated at $t_{0}$ increases in synchronism with the increasing number of modulation periods. Such increase of the intensity implies that QPM is successfully achieved due to a correctly chosen modulation period. However, the harmonic intensity generated at the other times of the drive pulse (red and blue curves) oscillates between 0 and very low values along the medium length because for these generation times the modulation period is wrongly chosen. Among the black curves in Fig. $6.6\left(t=t_{0}\right)$, the highest harmonic intensity is found in Fig. 6.6 (a), where a full modulation is applied $(\kappa=1)$. This can be explained as follows: due to the absence of gas in the low-density regions, a full suppression of the 

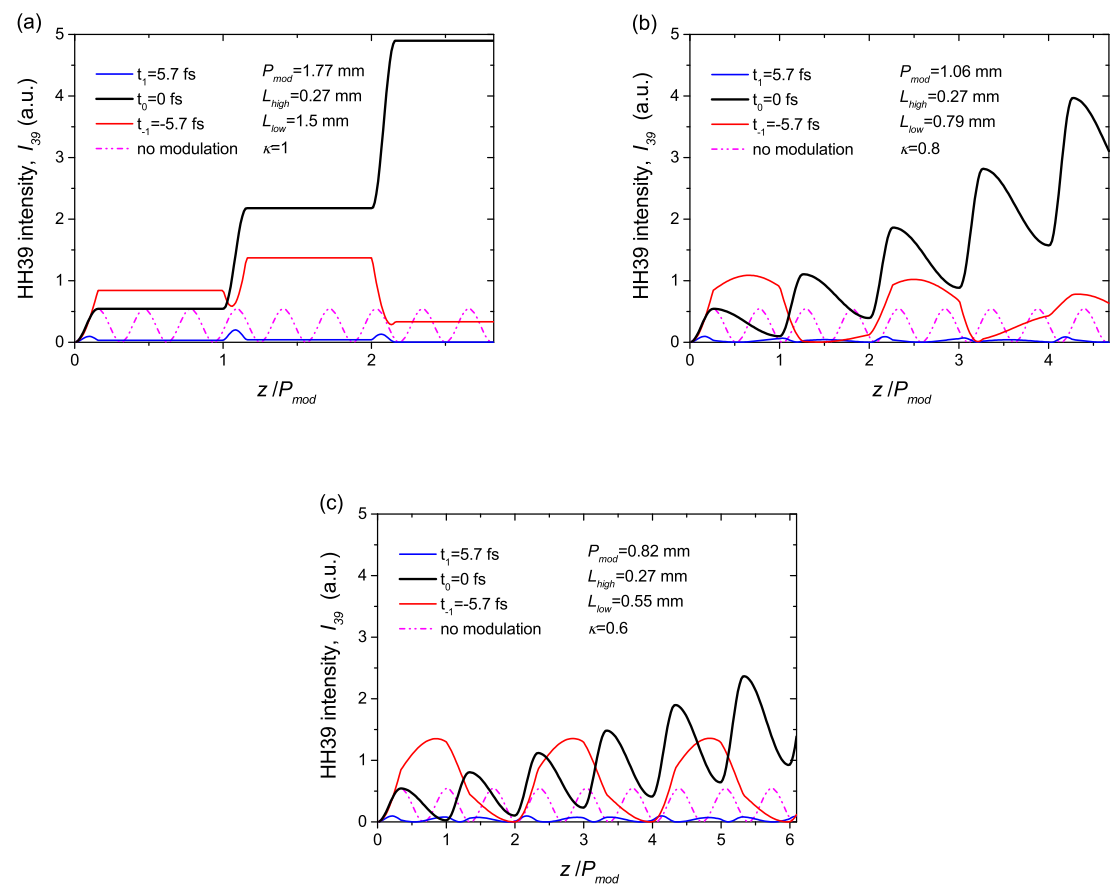

Figure 6.6: Growth of the on-axis $\mathrm{HH}$ intensity, $I_{39}$, throughout the density modulated medium for (a) $\kappa=1$, (b) $\kappa=0.8$ and (c) $\kappa=0.6$ when the modulation period, $P_{\text {mod }}$, is chosen to induce QPM at the peak of the drive laser pulse $\left(t_{0}=0 \mathrm{fs}\right)$. The black curves show the growth of $I_{39}$ generated at the drive laser peak, while the other curves show the growth of $I_{39}$ at an earlier and later time in the pulse $\left(t_{1}=-5.7 \mathrm{fs}\right.$ (red) and $t_{3}=5.7 \mathrm{fs}$ (blue)). The propagation coordinate is expressed relative to the modulation period. The pink dashed line represents $I_{39}$ generated at $t_{0}$ in a homogeneous medium, without density modulation $(\kappa=0)$.

out-of-phase generation is achieved. For other modulation depths, the $39^{\text {th }}$ harmonic generated from the remaining gas in the low-density region will still partially destructively interfere with the harmonics generated in the high-density region. Moreover, it can be noted that the maximum $I_{39}$, generated earlier in the pulse (at $t_{-1}$, red curves) is always higher than that generated with the same intensity later in the pulse (at $t_{1}$, blue curves), although the harmonic dipole amplitude, $d_{39}$, is the same at both times. This is due to a larger number of neutral atoms contributing to the HHG in the leading edge of the pulse than that in the trailing edge, because the ionization fraction reduces the neutral atomic density.

The results above show that choosing a particular modulation period only maximizes the $\mathrm{HH}$ output for a certain temporal fraction of the drive laser pulse, but, that also at other time intervals there is output, particularly in the leading edge. In an experiment, however, one is usually interested in the $\mathrm{HH}$ pulse energy, while the instantaneous $\mathrm{HH}$ intensity is rather difficult to monitor [152]. To optimize the pulse energy, $F_{q}$, we inte- 
grate the instantaneous harmonic intensity, $I_{q}(t)$, over the entire drive laser pulse and vary the modulation period. Since, from the previous calculations, the strongest growth in the instantaneous harmonic intensity is found in a fully density modulated medium $(\kappa=1)$, we restrict ourselves to this example. In previous experiments, generally a modulation period has been selected that is equal to the sum of the coherence lengths calculated for high-density and low-density regions at the time that the drive laser pulse reaches the peak intensity. The reason is that there the highest harmonic dipole amplitude, $d_{39}$, is achieved $[39,93,153]$. However, this choice appears questionable because then the growth of the harmonic intensity is placed in the time of the highest ionization rate (quickest change of plasma dispersion) which yields the shortest possible time interval of quasi-phase matching. On the other hand, if QPM is set to occur over a longer time interval (when the ionization rate is not maximum), one has to take into account a smaller instantaneous intensity. To determine the optimal modulation period for the highest on-axis harmonic pulse energy, we again apply the same loose focusing configuration for the experiment described in Section 6.3 (a drive laser peak intensity $I_{0}=2.5 \times 10^{14} \mathrm{~W} / \mathrm{cm}^{2}$, a Rayleigh range $z_{R}=18 \mathrm{~mm}$ achieved by a f/150 focusing lens, $L_{\text {med }}=5 \mathrm{~mm}$ ) and an argon gas pressure of $30 \mathrm{mbar}$. We calculate the harmonic pulse energy for the $39^{\text {th }}$ harmonic by selecting different modulation periods that provide QPM at various different time intervals of sufficiently high intensity (green area in Fig. 6.5). For more clarity, we have enlarged the green area and re-plotted it in Fig. 6.7 (a). It can be seen that between $t_{-1}$ and $t_{1}$, the coherence length in the low-density regions $\left(L_{c o h}^{\text {low }}\right)$ remains independent of time due to the absence of gas that can be ionized, while the coherence length in the high-density regions $\left(L_{c o h}^{\text {high }}\right)$ decreases due to the increasing ionization fraction. Here, we choose various different modulation periods that provide QPM at seven different instances in the drive pulse: at five instances in the leading edge, denoted as $P_{\text {mod }}\left(t_{-1}\right), P_{\text {mod }}\left(t_{-2}\right), P_{\text {mod }}\left(t_{-3}\right), P_{\text {mod }}\left(t_{-4}\right)$ and $P_{\text {mod }}\left(t_{0}\right)$, respectively, and at another two instances in the trailing edge, denoted as $P_{\text {mod }}\left(t_{2}\right)$ and $P_{\text {mod }}\left(t_{1}\right)$. The calculated lengths for the high-density and low-density regions as well as the corresponding modulation periods are listed in Table 6.1. In Fig. 6.7 (b), we show the calculated on-axis

Table 6.1: List of coherence lengths in the high-density and low-density regions and the modulation periods $\left(P_{\text {mod }}\right)$ chosen to provide QPM at seven different instances within the drive laser pulse when the $39^{\text {th }}$ harmonic is generated.

\begin{tabular}{|c|c|c|c|}
\hline & $L_{\text {high }}(\mathrm{mm})$ & $L_{\text {low }}(\mathrm{mm})$ & $P_{\text {mod }}(\mathrm{mm})$ \\
\hline$t_{-1}=-5.7 \mathrm{fs}$ & 0.63 & 1.50 & 2.13 \\
\hline$t_{-2}=-4.2 \mathrm{fs}$ & 1.98 & 1.50 & 1.98 \\
\hline$t_{-3}=-2.8 \mathrm{fs}$ & 0.39 & 1.50 & 1.89 \\
\hline$t_{-4}=-1.4 \mathrm{fs}$ & 0.32 & 1.50 & 1.82 \\
\hline$t_{0}=0 \mathrm{fs}$ & 0.27 & 1.50 & 1.77 \\
\hline$t_{2}=2.8 \mathrm{fs}$ & 0.20 & 1.50 & 1.70 \\
\hline$t_{1}=5.7 \mathrm{fs}$ & 0.17 & 1.50 & 1.67 \\
\hline
\end{tabular}

harmonic pulse energy, $F_{39}$, versus the propagation coordinate through the medium for the listed modulation periods. For comparison with the case where QPM is absent, we calculated the $\mathrm{HH}$ pulse energy from a homogeneous medium as well. It can be seen 

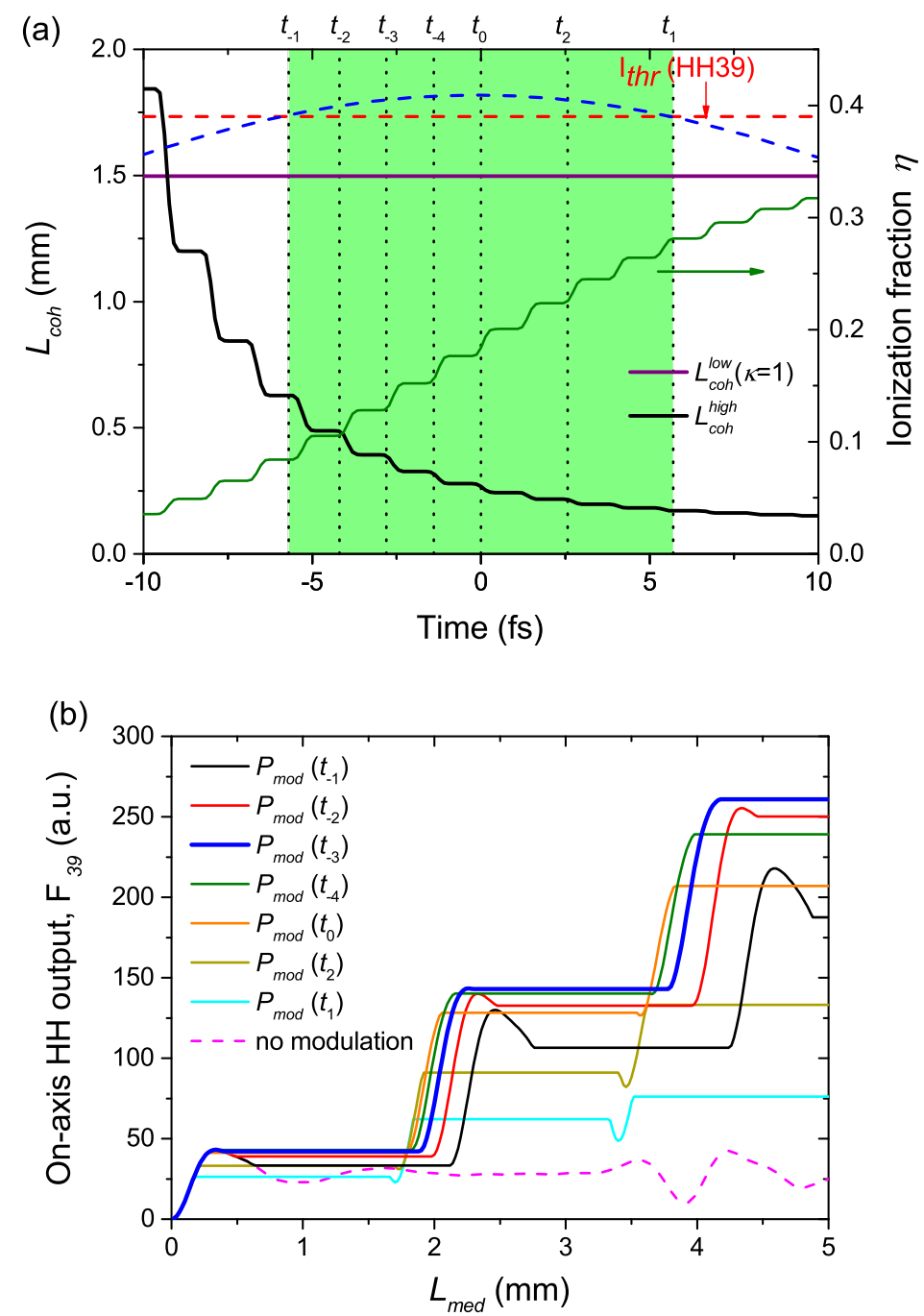

Figure 6.7: (a) calculated coherence lengths enlarged from Fig. 6.5. The purple horizontal line is the coherence length in the low-density regions, while the black curve is the coherence length in the high-density regions. The modulation depth is set to $\kappa=1$. For the calculation of the HH output pulse energy, seven different modulation periods are chosen corresponding to providing QPM at seven selected instances in the drive laser pulse (from $t_{-1}$ to $t_{1}$, dotted lines). (b) On-axis HH pulse energy for the $39^{\text {th }}$ harmonic versus the length of the medium obtained with the seven different modulation periods. The highest output is achieved with $P_{\text {mod }}$ chosen to provide transient QPM in the rising edge of the pulse $\left(t_{-3}\right.$, blue line). The pink dashed line of the lowest output is obtained without density modulation.

that the output pulse energy is very sensitive to the chosen modulation period. For a homogeneous medium (without density modulation, pink dashed curve), the output oscil- 
lates along the medium length and reaches only a small value. In the density modulated medium, the output grows stepwise along the propagation direction with each modulation period. However, the most interesting and important finding from Fig. 6.7 (b) is that the highest on-axis harmonic pulse energy is obtained by applying a modulation period that provides transient QPM in the leading edge rather than at the peak intensity of the drive laser pulse, here with $P_{\text {mod }}\left(t_{-3}\right)$. Choosing such a modulation period results in about $25 \%$ more output pulse energy generated in the medium.

In summary, the optimum modulation period for the highest on-axis $\mathrm{HH}$ pulse energy, $F_{39}$, cannot simply be determined from the remaining wave-vector mismatch at the peak of the drive laser pulse, although at this time the highest instantaneous output intensity is reached. We address this to the circumstance that at the peak also the time interval of successful QPM is the shortest.

\subsection{CONCLUSION}

We have developed a one-dimensional, dynamic model for quasi-phase matching (QPM) of high-order harmonic generation. The model is used to calculate the optimum quasiphase-matching period, using the example of generation in a medium (argon) with spatially periodic density modulation (periodic structure). Due to the ultrafast temporal dependence of the coherence length in high-order harmonic generation at elevated strongly ionizing intensities, which are required for short output wavelength, quasi-phase matching occurs transiently for any selected generated harmonic at some instance during the drive laser pulse. The choice of the modulation period determines the specific moment and duration of quasi-phase matching. We show that simply choosing the modulation period to provide phase matching at the peak intensity of the pulse, when the highest amplitude of the nonlinearly induced high-order harmonic dipole amplitude is reached, does not provide the highest harmonic output pulse energy. To find the optimum modulation period, it is essential to analyze the time dependence of the wave-vector mismatch and the harmonic dipole amplitude along the entire pulse duration. According to our model, the optimal modulation is obtained when transient quasi-phase matching is provided in the leading edge of the pulse.

Although we have presented results from the model using a specific choice of parameters for an idealized situation, we note that the model can also be applied to more complicated modulation profiles such as profiles measured in our density modulated cluster jet mentioned in Chapter 1 . The model may also be applied to more complicated gas distributions such as multi dual-gas jet arrays [45], by recalculating the wave-vector mismatch and the induced dipole amplitudes for the different gas species used. Further improvements of this model would include an extension to three dimensions (including the transverse direction) together with an improved quantum mechanical calculation of the single-atom response. 


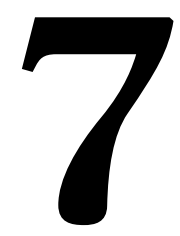

CONCLUSION AND OUTLOOK 
Light sources based on high-order harmonic generation (HHG) generate extreme ultraviolet (XUV) radiation, and even soft X-rays, with a high, unprecedented spatial and temporal coherence. These unique properties enable new applications such as nanometer resolved coherent diffraction imaging [29] and femtosecond or even attosecond resolved detection of electron dynamics in atoms [9]. However, HHG is intrinsically inefficient and the highest-ever achieved efficiency is rather modest (up to $10^{-5}$ ) and was only achieved in a limited spectral region of the high-order harmonic $(\mathrm{HH})$ spectrum where the phase-matching condition could be fulfilled. The highest harmonic order (shortest wavelength) that can be efficiently generated is limited through the loss of the phasematching condition due to a large ionization fraction induced by the required high drive laser intensities. A potential solution to efficiently generate $\mathrm{HH}$ radiation at shorter wavelengths is using quasi-phase matching (QPM). For achieving QPM, a spatial modulation of the generation process along the entire medium has to be introduced, enabling HHG in the in-phase regions and suppressing HHG in the out-of-phase regions.

A promising scheme for achieving QPM in HHG is to use a density modulated cluster jet. Such a cluster jet can be produced from a supersonic expansion of a noble gas through a slit nozzle, where clusters are formed via van-der-Waals aggregation. Compared to a gas jet, a density modulation is expected to be more easily achieved, by placing an array of wires (grid) on the top of the nozzle. The reason for this is that clusters as compared to gas atoms (monomers) exhibit a much reduced transverse diffusion due to their relatively large mass, which is promising to create a rather high density contrast behind the grid. Thereby also a finer-scale modulation behind an array of wires appears achievable, which can be important for increasing the efficiency via QPM. Another advantage of clusters might be that they exhibit a higher nonlinear $\mathrm{HH}$ response than gas atoms, as claimed by others $[47,48]$.

However, in initial experiments, carried out by us with a modulated cluster jet as shown in Fig. 1.1, we did not observe any enhanced HH output using various different modulation periods. These experiments suggest that, even if using a density modulated cluster jet remains promising for achieving QPM in HHG, an according demonstration first requires a thorough investigation of several basic and essential aspects.

These aspects are, first, the liquid mass fraction, $g$, which describes the relative percentage of the number of atoms aggregated in clusters. This fraction is generally assumed to be equal to unity without further justification. However, the actual value of the liquid mass fraction, $g$, according to our work, lies below $40 \%$, even at a very low reservoir temperature (173 K, via cooling by pre-cooled nitrogen gas) and a high stagnation pressure (50 bar). Therefore, there is generally an overestimation of the average cluster size and the density in the jet. Secondly, as a consequence of using wrongly determined values for $g$, the interpretation of the nonlinear $\mathrm{HH}$ response of clusters from previous HHG experimental data appears quite questionable. Finally, the variation of phase mismatch on an ultrashort time scale, due to the rapidly increasing ionization fraction in the generating medium during the drive laser pulse in previous calculations and experiments, has not yet been taken into account for selecting the proper modulation period for QPM. A particular, selected modulation period may enable a maximum generation of high-order harmonics only for an extremely short time interval. However, the total, time-integrated $\mathrm{HH}$ yield, i.e., the pulse energy, is not necessarily maximized. 
In this thesis, we investigated the fundamental issues regarding these three essential aspects for applying density modulated cluster jets for quasi-phase matching in highorder harmonic generation. These aspects need to be investigated before an experimental demonstration of QPM becomes possible for an efficient generation of shortwavelength radiation via HHG.

- The first issue we investigated is related to the process of cluster formation in a supersonic slit nozzle, and it aimed on determining the average size, $\langle N\rangle$, of clusters, as well as the liquid mass fraction, $g$, in the jet. In Chapter 4 , we presented a comprehensive modelling of cluster formation and systematically investigated all influences of various critical physical assumptions for the gas condensation in a supersonic nozzle using argon as an example. The baseline theoretical model as proposed in Chapter 4, uses a thermal non-equilibrium surface tension model, a saturated liquid density, a non-equilibrium cluster temperature, and Hill's average cluster radius which is computed from the moments of the size distribution. Using this baseline model, we showed that the liquid mass fraction is very insensitive with regards to any variations in the model which justifies the usage of these data to derive the averaged cluster size from experimental data. The calculated liquid mass fraction, $g$, from the baseline model is then used to retrieve the average cluster size, $\langle N\rangle$, from interferometric and Rayleigh scattering measurements. We determined that the average cluster size, $\langle N\rangle$, follows a modified power law for higher Hagena parameters, $\Gamma^{*}$, in the range of $1.8 \times 10^{4}$ to $2.5 \times 10^{5}$. Our power law complements the previously found scaling law for the Hagena parameter, $\Gamma^{*}$, extending it into the region in a consistent fashion, i.e., extends the total range of predictable average cluster size from $\Gamma^{*}=10^{3}$ to $2.5 \times 10^{5}$. This translates into a range of the average cluster size, $\langle N\rangle$, that can be predicted with high reliability, extending from about a thousand to almost ten million atoms per cluster. The derived average cluster size and liquid mass fraction are essential for interpreting the measurements on HHG from the cluster jet to determine the single-atom response of clusters as discussed in Chapter 5.

- Secondly, we performed a detailed experimental study on HHG in a supersonic argon jet, as described in Chapter 5 . In order to identify and separate the contributions from clusters versus that of gas monomers in the jet to the generation of high-order harmonics, we characterized the harmonic spectra over a broad range of stagnation pressures (between 300 mbar to 35 bar) at two different reservoir temperatures ( $303 \mathrm{~K}$ and $363 \mathrm{~K}$ ). Varying the temperature allowed to maintain the same average cluster size, $\langle N\rangle$, at different total atomic number densities in the jet. We proposed a simple model to interpret the dependence of the $\mathrm{HH}$ yield on the total atomic number density in the jet. Using the calculated value of $g$, which is below $20 \%$ for our experimental conditions, we derived the relative single-atom response of clusters with different average sizes. We observed that, below an average cluster size, $\langle N\rangle$, of about 1000 atoms per cluster, HHG in clusters shows the same efficiency as in gas monomers. Only with larger clusters $(\langle N\rangle>1000$ atoms per cluster), HHG becomes less efficient. For generating efficient high-order harmonics, it becomes therefore important to accurately control the average cluster size. In the measured $\mathrm{HH}$ spectra, we observed no changes of the cut-off energy, which 
indicates that the three-step model for HHG in atoms remains valid for HHG in clusters. Specifically, the tunnel ionized electrons recombine with their parent ions instead of their neighbouring ions, as suggested in other literature [48, 57].

- Lastly, we studied quasi-phase matching for HHG, specifically aiming at determining a proper quasi-phase matching modulation period for achieving the optimum harmonic output pulse energy. We developed a one-dimensional, dynamic QPM model for HHG in a gaseous medium (argon) with spatially periodic density modulation, as presented in Chapter 6. From the model, we found that it is not possible to achieve QPM for any selected generated high-order harmonics during the entire drive laser pulse using a fixed periodic structure. This is due to the ultrafast temporal dependence of the coherence length (wave-vector mismatch) at the elevated, strongly ionizing intensities required for generating short output wavelengths. We showed that simply choosing the modulation period according to the coherence length calculated at the peak intensity of the laser pulse when the highest harmonic dipole amplitude, $d_{q}$, is reached, is not the optimum choice for achieving the highest harmonic output pulse energy. In order to find the optimum modulation period, it is essential to analyze the wave-vector mismatch and harmonic dipole amplitude during the entire drive pulse. According to our model, the optimum HH output pulse energy is obtained when transient QPM is provided in the leading edge of the drive laser pulse.

The basic understanding of the cluster formation, the nonlinear HH response of clusters and the temporal dependence of the phase mismatch in the generating media is essential, and the progress achieved in our work on these aspects forms a solid and important foundation towards the further development of QPM in HHG. Yet, to determine whether quasi-phase matched HHG at shorter wavelengths is feasible by introducing a periodic density modulation in a mixture of clusters and gas monomers, one requires further developments including a better understanding of the dynamics of gas monomers and clusters, and a detailed analysis of the density modulation behind obstacles in a mixed gas and cluster jet.

Following the three major issues which have been investigated in this thesis, future research might be carried out along the following lines

- A first essential step towards experimentally demonstrating QPM in HHG is to design an array of wires (grid) with a proper modulation period from our QPM model described in Chapter 6. However, before this step can be done, interferometric techniques need to be carried out that quantify the actual modulated density profile of the cluster jet behind the grid to check the efficiency improvements obtained with QPM. These investigations might lead to further experimental modifications such as reducing the temperature of the nozzle for a higher density contrast or modifying the cross-sections of the bars in the grid.

- To obtain a more accurate predication of the average cluster size, $\langle N\rangle$, the current one-dimensional model might have to be improved further. The motivation for this is that there is still, so far, a certain offset of about one order of magnitude 
between the computed and experimental values of $\langle N\rangle$, even using our baseline model. An option would be a three-dimensional model based on fully solving the Becker-Döring equations [67] for more accurately describing the gas condensation in arbitrary shaped nozzles. Another shortcoming of the present model and cluster jet characterization is that it focuses only at the very exit of the nozzle, while HHG is to be performed further downstream, behind a grid. There the influence of placing an array of wires on both clusters and gas monomers, e.g., the influence of shock formation, has not been investigated so far. Questions such as whether clusters can pass without disintegration through the shock remain so far unanswered.

- This thesis focused only on cluster jets consisting of noble gas atoms for HHG. However, there are also other nanoparticles having similar sizes, i.e., of the order of few nanometers, such as buckyballs $\left(C_{60}\right)$ or metal nanoparticles, which may be promising as well. For instance, silver nanoparticles have been demonstrated to exhibit an appreciable nonlinear HH response [154] and can be injected into a supersonic gas flow via an electrospray aerosol generator. Furthermore, using elliptical nanoparticles may serve to locally enhance the driving field [155, 156], such that relatively low incident intensities could be used to generate high-order harmonics efficiently. With such an approach large-frame drive laser systems could be replaced by single femtosecond laser oscillators with a pulse energy of only a few tens of nJ and a much higher repetition rate of several tens of megahertz. Recently, a theoretically study showed that a periodic modulation of the ellipsoidal sliver nanoparticle concentration in a gas flow is also promising for providing QPM in HHG [157]. 



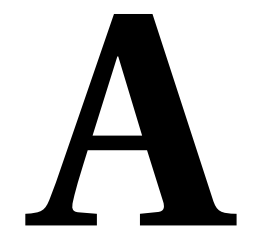

NOZZLE DESIGN 


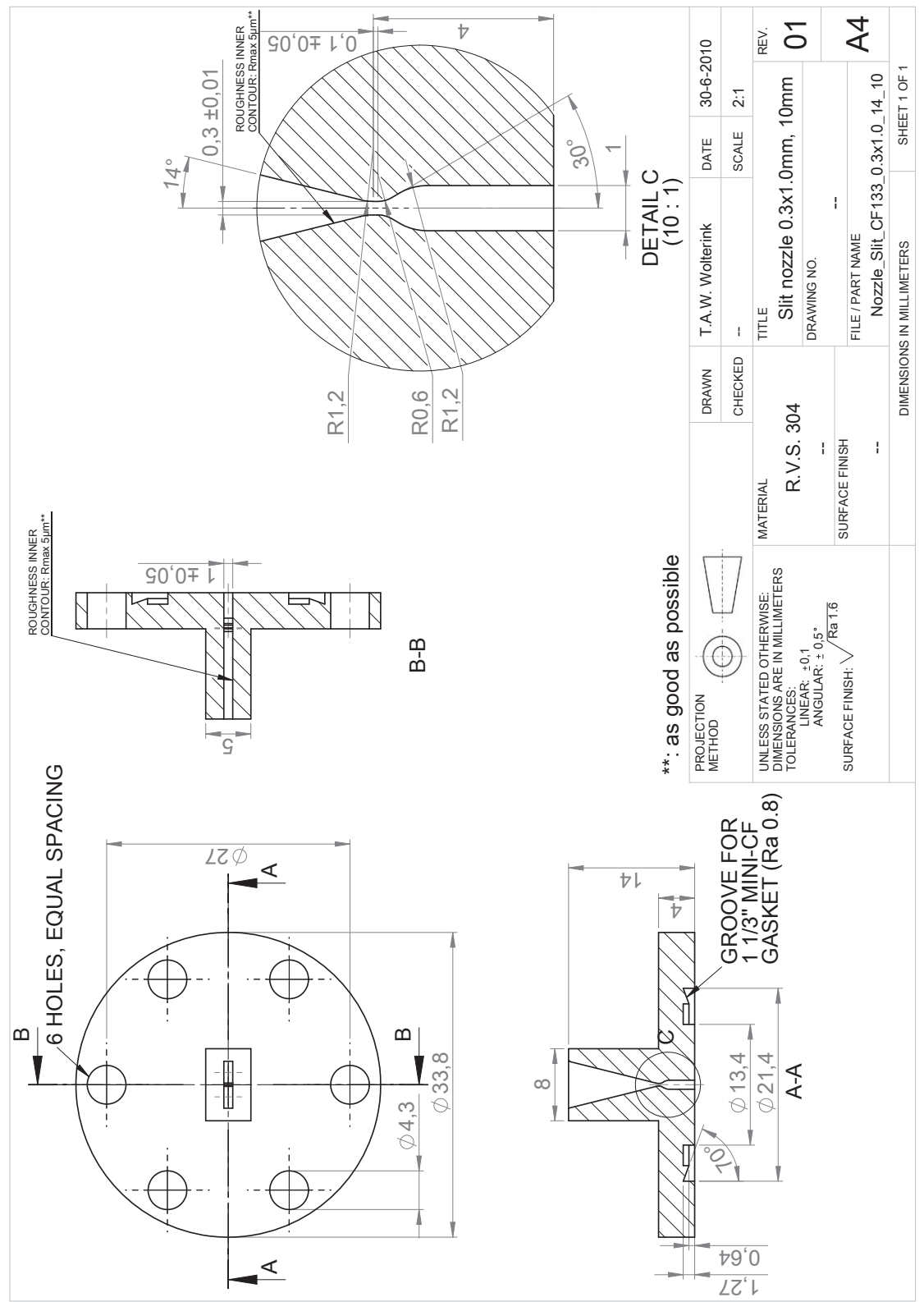

Figure A.1: Slit nozzle designed for this thesis (Planar nozzle, slit area: $1.0 \times 5.0 \mathrm{~mm}^{2}$, throat size: $0.3 \times 1.0 \mathrm{~mm}^{2}$, expansion half-angle: $14^{\circ}$ ). [64] 


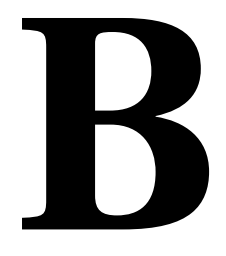

\section{HHG SETUP}




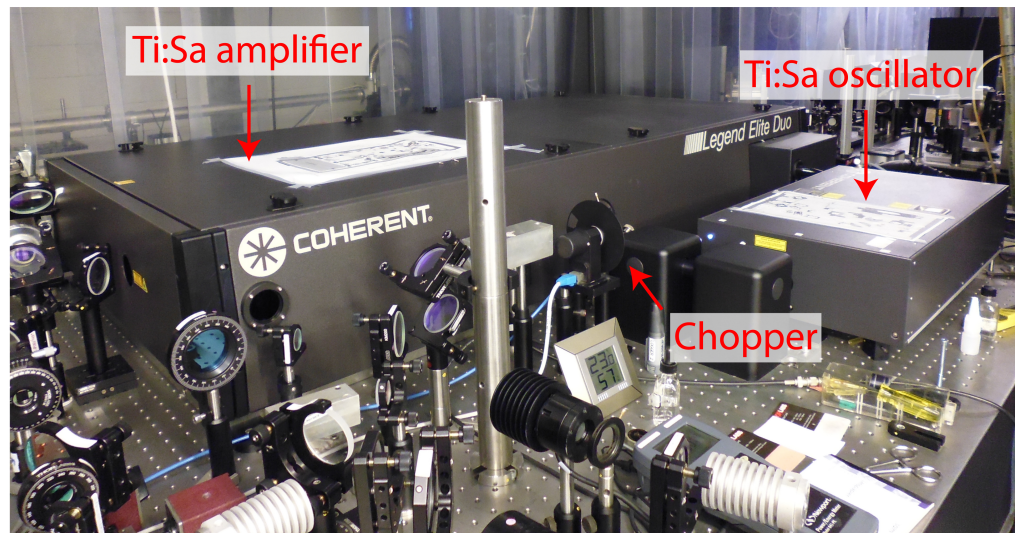

Figure B.1: A photograph of the high power Ti:Sa laser system.

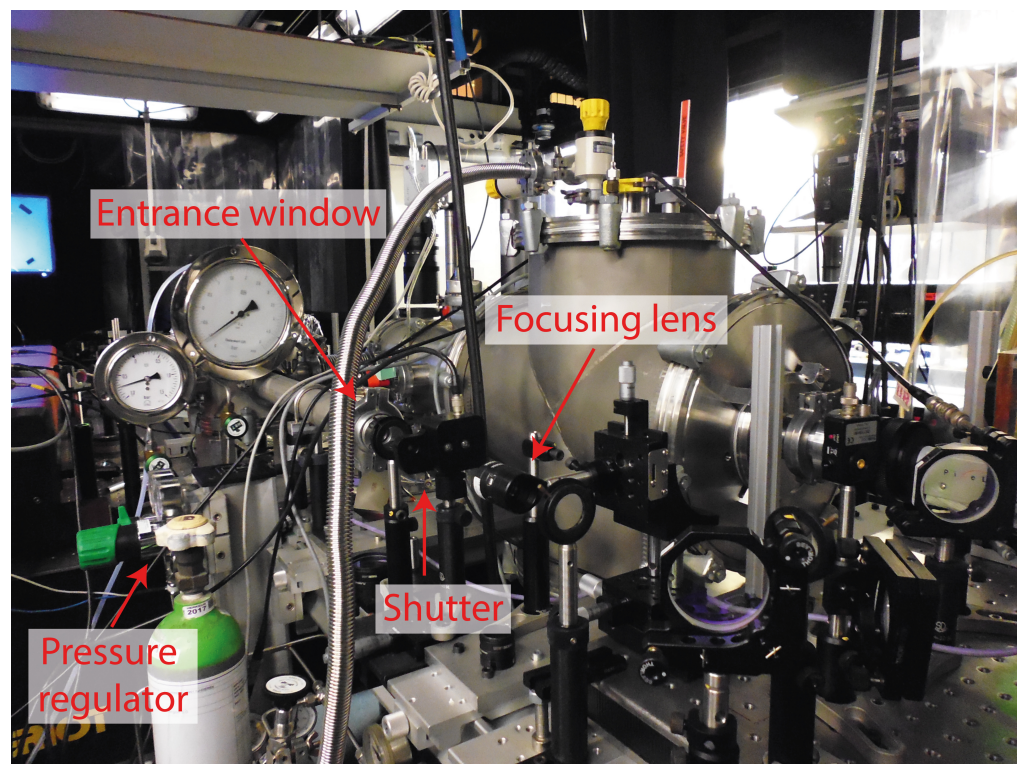

Figure B.2: A photograph of the beam focusing setup and pressure regulation system. 


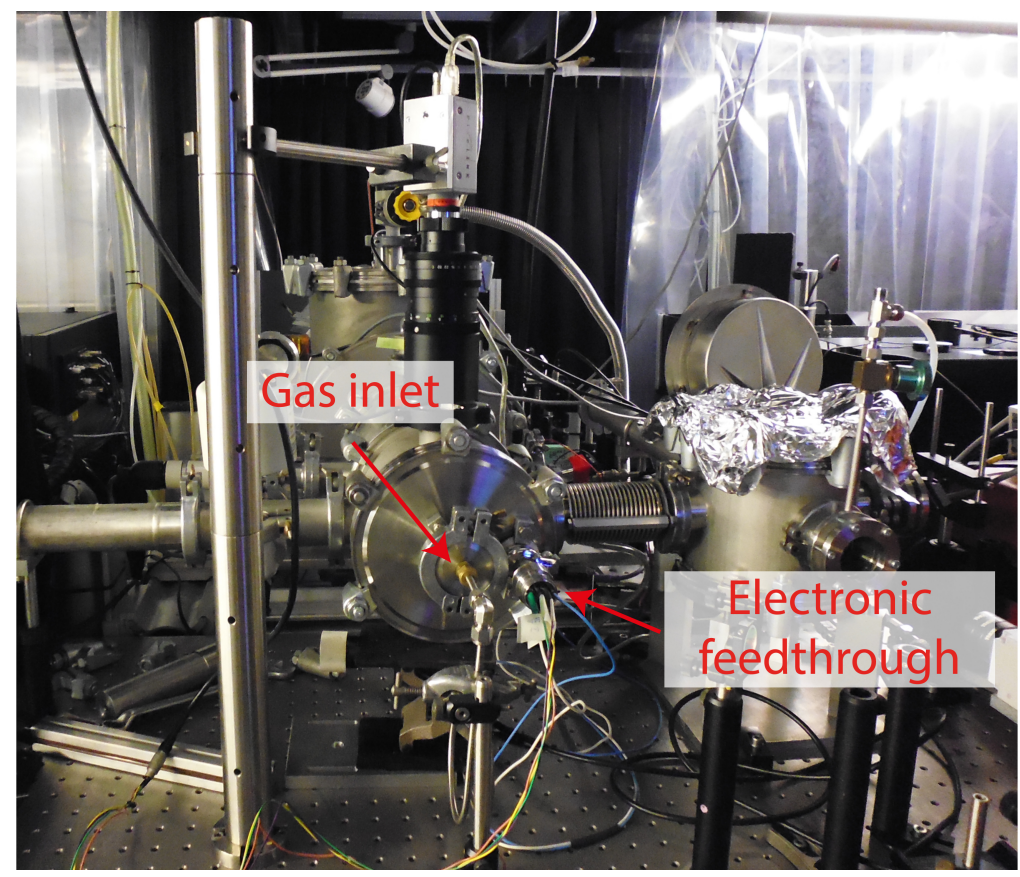

Figure B.3: A photograph of the vacuum chamber for mounting the supersonic nozzle.

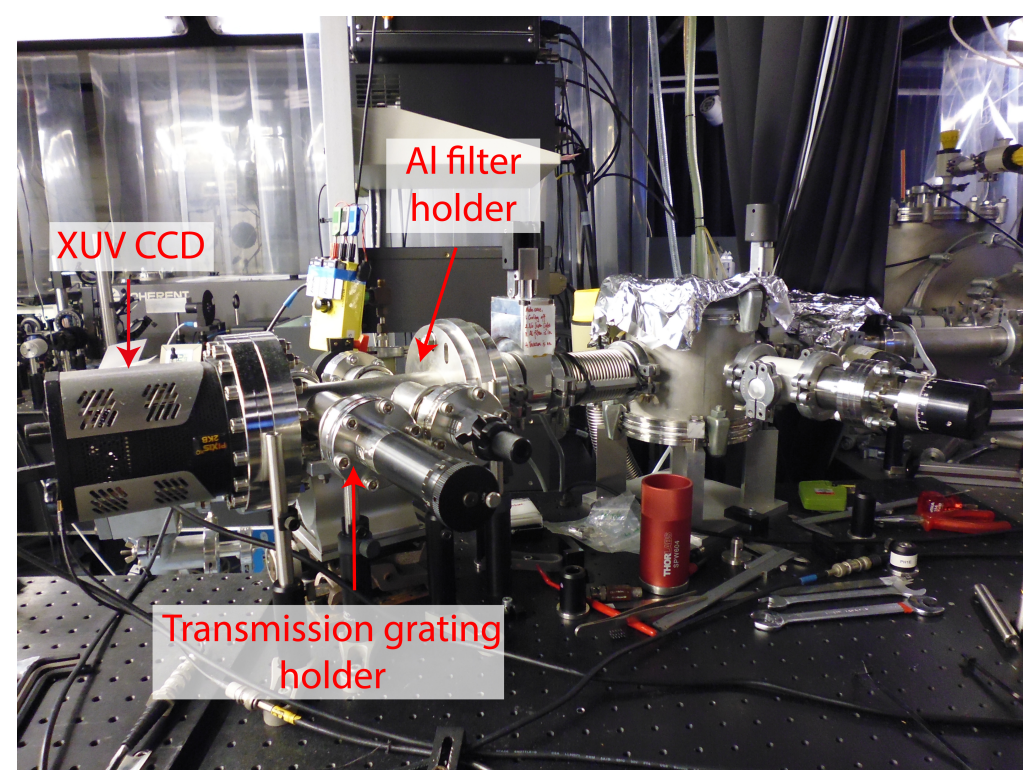

Figure B.4: A photograph of the home-built XUV spectrometer. 



\section{C}

\section{SOLUTION METHOD FOR CONSERVATION EQUATIONS}

Using the equations of state, Eqs. (4.18) and (4.19) in Chapter 4, the material derivatives of $p$ and $e$ become:

$$
\frac{D p}{D t}=-\frac{\rho^{2}}{1-g} \beta_{\rho} \frac{D g}{D t}+\rho \beta_{\rho} \frac{D \rho}{D t}+\rho \beta_{T} \frac{D T}{D t},
$$

and

$$
\frac{D e}{D t}=\left(c_{\nu}+g R\right) \frac{D T}{D t}+(R T-L) \frac{D g}{D t}
$$

where

$$
\begin{aligned}
& \beta_{\rho}=\frac{1}{\rho}\left[\frac{p}{\rho}+(1-g) \frac{p}{z}\left(\frac{\partial z}{\partial \rho}\right)_{T}\right], \\
& \beta_{T}=\frac{1}{\rho}\left[\frac{p}{T}+\frac{p}{z}\left(\frac{\partial z}{\partial T}\right)_{\rho}\right] .
\end{aligned}
$$

The compressibility factor $z(\rho, T)$ is computed by employing the series expansion described in Reid et al. [158]:

$$
z(\rho, T)=1+B(T)\left(\frac{\rho}{M}\right)+C(T)\left(\frac{\rho}{M}\right)^{2}+\ldots
$$

which we truncate after the third term. In this equation $M$ is the molar mass and $B$ and $C$ are the second and third virial coefficients, respectively, which are modeled by using the equations provided by Stewart and Jacobsen [159]:

$$
B(T)=\sum_{i=1}^{5} B_{i} T^{b_{i}}, \quad C(T)=\sum_{i=1}^{6} C_{i} T^{c_{i}},
$$




\begin{tabular}{|c|c|c||c|c|c|}
\hline$i$ & $B_{i}$ & $b_{i}$ & $i$ & $C_{i}$ & $c_{i}$ \\
\hline 1 & $0.2866924170 \times 10^{-1}$ & 0 & 1 & $0.2850918168 \times 10^{6}$ & $-11 / 4$ \\
2 & $-0.3554066483 \times 10^{2}$ & $-5 / 4$ & 2 & $-0.1472740048 \times 10^{9}$ & $-7 / 2$ \\
3 & $-0.8003312290 \times 10^{2}$ & $-3 / 2$ & 3 & $0.6616737314 \times 10^{9}$ & $-15 / 4$ \\
4 & $-0.1388893486 \times 10^{11}$ & $-11 / 2$ & 4 & $-0.1262999051 \times 10^{11}$ & $-9 / 2$ \\
5 & $0.3663978029 \times 10^{11}$ & $-23 / 4$ & 5 & $0.3794222032 \times 10^{12}$ & $-21 / 4$ \\
6 & $-0.6465333262 \times 10^{12}$ & $-11 / 2$ \\
\hline
\end{tabular}

Table C.1: Coefficients in second and third virial coefficients

With these expressions we write Eqs. (4.12), (4.13) and (4.17) as

$$
K \frac{D}{D t}\left(\begin{array}{c}
\rho \\
u \\
T
\end{array}\right)=\left(\begin{array}{c}
-\frac{1}{A} \frac{D A}{D t} \\
\frac{\rho^{2}}{1-g} \beta_{\rho} \frac{D g}{D t} \\
(R T-L) \frac{D g}{D t}
\end{array}\right),
$$

where $K$ is a matrix:

$$
K=\left(\begin{array}{ccc}
\frac{1}{\rho} & \frac{1}{u} & 0 \\
\beta_{\rho} & u & \beta_{T} \\
\frac{p}{\rho^{2}} & 0 & -\left(c_{\nu}+g R\right)
\end{array}\right) .
$$

The system Eq. (C.7) can be solved for $\rho, u$ and $T$ when the right hand side is given. The term $-\frac{1}{A} \frac{D A}{D t}$ is determined by the nozzle geometry so we are left with $\frac{D g}{D t}$ which is obtained by differentiating Eq. (4.21)

$$
\frac{D g}{D t}=\frac{D}{D t}\left(\frac{4}{3} \pi \rho_{L} \hat{\mu}_{3}\right) \approx \frac{4}{3} \pi \rho_{L} \frac{D \hat{\mu}_{3}}{D t} .
$$

The moment transport equations Eq. (4.23), the system Eq. (C.7) and finally Eq. (C.9) form a closed set of differential equations that can be solved in the diverging supersonic part of the nozzle in a downstream space-marching manner. The required throat conditions are obtained by integrating the energy equation Eq. (4.14) and the entropy equation Eq. (4.17) from reservoir conditions to sonic conditions. 


\section{REFERENCES}

[1] A. L. Schawlow and C. H. Townes, Infrared and optical masers, Phys. Rev. 112, 1940 (1958).

[2] T. H. Maiman, Stimulated optical radiation in ruby, Nature 187, 493 (1960).

[3] P. A. Franken, A. E. Hill, C. W. Peters, and G. Weinreich, Generation of optical harmonics, Phys. Rev. Lett. 7, 118 (1961).

[4] S. J. Brosnan and R. L. Byer, Optical parametric oscillator threshold and linewidth studies, IEEE J. Quant. Electron. 15, 415 (1979).

[5] P. Monot, T. Auguste, P. Gibbon, F. Jakober, G. Mainfray, A. Dulieu, M. LouisJacquet, G. Malka, and J. L. Miquel, Experimental demonstration of relativistic self-channeling of a multiterawatt laser pulse in an underdense plasma, Phys. Rev. Lett. 74, 2953 (1995).

[6] G. H. C. New and J. F. Ward, Optical third-harmonic generation in gases, Phys. Rev. Lett. 19, 556 (1967).

[7] Y. Mori, I. Kuroda, S. Nakajima, T. Sasaki, and S. Nakai, New nonlinear optical crystal: cesium lithium borate, Appl. Phys. Lett. 67, 1818 (1995).

[8] D. Z. Kandula, C. Gohle, T. J. Pinkert, W. Ubachs, and K. S. E. Eikema, Extreme ultraviolet frequency comb metrology, Phys. Rev. Lett. 105, 063001 (2010).

[9] E. Goulielmakis, Z. H. Loh, A. Wirth, R. Santra, N. Rohringer, V. S. Yakovlev, S. Zherebtsov, T. Pfeifer, A. M. Azzeer, M. F. Kling, S. R. Leone, and F. Krausz, Realtime observation of valence electron motion, Nature 466, 739 (2010).

[10] F. Lepine, M. Y. Ivanov, and M. J. J. Vrakking, Attosecond molecular dynamics: fact or fiction? Nat. Photon. 8, 195 (2014).

[11] J. J. Rocca, Table-top soft x-ray lasers, Rev. Sci. Instrum 70, 3799 (1999).

[12] M. Bayraktar, F. A. Van Goor, K. J. Boller, and F. Bijkerk, Spectral purification and infrared light recycling in extreme ultraviolet lithography sources, Opt. Express 22, 8633 (2014).

[13] J. F. Kephart, R. P. Godwin, and G. H. McCall, Bremsstrahlung emission from laser - produced plasmas, Appl. Phys. Lett. 25, 108 (1974).

[14] P. Nicolosi, E. Jannitti, and G. Tondello, Soft x-ray emission of continua from laser produced plasmas, Appl. Phys. B 26, 117 (1981). 
[15] S. Sebba, R. Haroutunia, P. Balcou, G. Grillon, A. Rousse, S. Kazamias, T. Marin, J. P. Rousseau, L. Notebaert, M. Pittman, J. P. Chambaret, A. Antonetti, D. Hulin, D. Ros, A. Klisnick, A. Carillon, P. Jaeglé, G. Jamelot, and J. F. Wyart, Saturated amplification of a collisionally pumped optical-field-ionization soft $x$-ray laser at 41.8 nm, Phys. Rev. Lett. 86, 3004 (2001).

[16] H. Tanaka, A. Matsumoto, K. Akinaga, A. Takahashi, and T. Okada, Comparative study on emission characteristics of extreme ultraviolet radiation from co2 and nd:yag laser-produced tin plasmas, Appl. Phys. Lett. 87, 041503 (2005).

[17] D. H. Bilderback, P. Elleaume, and E. Weckert, Review of third and next generation synchrotron light sources, J. Phys. B 38, S773 (2005).

[18] W. A. Hendrickson, Determination of macromolecular structures from anomalous diffraction of synchrotron radiation, Science 254, 51 (1991).

[19] T. Ishikawa, H. Aoyagi, T. Asaka, Y. Asano, N. Azumi, T. Bizen, H. Ego, K. Fukami, T. Fukui, Y. Furukawa, and et al., A compact $x$-ray free-electron laser emitting in the sub-ångström region, Nat. Photon. 6, 540 (2012).

[20] S. Boutet and G. J. Williams, The coherent $x$-ray imaging (cxi) instrument at the linac coherent light source (lcls), New J. Phys. 12, 035024 (2010).

[21] B. Rudek, S. K. Son, L. Foucar, S. W. Epp, B. Erk, R. Hartmann, M. Adolph, R. Andritschke, A. Aquila, N. Berrah, and et al., Ultra-efficient ionization of heavy atoms by intense $x$-ray free-electron laser pulses, Nat. Photon. 6, 858 (2012).

[22] A. McPherson, B. D. Thompson, A. B. Borisov, K. Boyer, and C. K. Rhodes, Multiphoton-induced $x$-ray emission at 4-5 kev from xe atoms with multiple core vacancies, Nature 370, 631 (1994).

[23] A. McPherson, G. Gibson, H. Jara, U. Johann, T. S. Luk, I. A. McIntyre, K. Boyer, and C. K. Rhodes, Studies of multiphoton production of vacuum-ultraviolet radiation in the rare gases, J. Opt. Soc. Am. B 4, 595 (1987).

[24] M. Ferray, A. L’Huillier, X. F. Li, L. A. Lompre, G. Mainfray, and C. Manus, Multipleharmonic conversion of 1064-nm radiation in rare-gases, J. Phys. B 21, L31 (1988).

[25] A. L'Huillier, P. Balcou, S. Candel, K. J. Schafer, and K. C. Kulander, Calculations of high-order harmonic-generation processes in xenon at $1064 \mathrm{~nm}$, Phys. Rev. A 46, 2778 (1992).

[26] P. B. Corkum, Plasma perspective on strong field multiphoton ionization, Phys. Rev. Lett. 71, 1994 (1993).

[27] M. Lewenstein, P. Balcou, M. Y. Ivanov, A. L'Huillier, and P. B. Corkum, Theory of high-harmonic generation by low-frequency laser fields, Phys. Rev. A 49, 2117 (1994). 
[28] M. Schultze, E. M. Bothschafter, A. Sommer, S. Holzner, W. Schweinberger, M. Fiess, M. Hofstetter, R. Kienberger, V. Apalkov, V. S. Yakovlev, M. I. Stockman, and F. Krausz, Controlling dielectrics with the electric field of light, Nature 493, 75 (2013).

[29] E. R. Shanblatt, C. L. Porter, D. F. Gardner, G. F. Mancini, J. Karl, R. M., M. D. Tanksalvala, C. S. Bevis, V. H. Vartanian, H. C. Kapteyn, D. E. Adams, and M. M. Murnane, Quantitative chemically specific coherent diffractive imaging of reactions at buried interfaces with few nanometer precision, Nano Lett. 16, 5444 (2016).

[30] G. Lambert, B. Vodungbo, J. Gautier, B. Mahieu, V. Malka, S. Sebban, P. Zeitoun, J. Luning, J. Perron, A. Andreev, S. Stremoukhov, F. Ardana-Lamas, A. Dax, C. P. Hauri, A. Sardinha, and M. Fajardo, Towards enabling femtosecond helicitydependent spectroscopy with high-harmonic sources, Nat. Commun. 6, 6167 (2015).

[31] M. Nakasuji, A. Tokimasa, T. Harada, Y. Nagata, T. Watanabe, K. Midorikawa, and H. Kinoshita, Development of coherent extreme-ultraviolet scatterometry microscope with high-order harmonic generation source for extreme-ultraviolet mask inspection and metrology, Jpn. J. Appl. Phys. 51, 06FB09 (2012).

[32] G. Lambert, T. Hara, D. Garzella, T. Tanikawa, M. Labat, B. Carre, H. Kitamura, T. Shintake, M. Bougeard, S. Inoue, Y. Tanaka, P. Salieres, H. Merdji, O. Chubar, O. Gobert, K. Tahara, and M. E. Couprie, Injection of harmonics generated in gas in a free-electron laser providing intense and coherent extreme-ultraviolet light, Nat. Phys. 4, 296 (2008).

[33] J. F. Hergott, M. Kovacev, H. Merdji, C. Hubert, Y. Mairesse, E. Jean, P. Breger, P. Agostini, B. Carré, and P. Salières, Extreme-ultraviolet high-order harmonic pulses in the microjoule range, Phys. Rev. A 66, 021801 (2002).

[34] S. J. Goh, J. Reinink, Y. Tao, P. J. M. van der Slot, H. M. J. Bastiaens, J. L. Herek, S. G. Biedron, S. V. Milton, and K. J. Boller, Spectral control of high-harmonic generation via drive laser pulse shaping in a wide-diameter capillary, Opt. Express 24, 1604 (2016).

[35] E. Takahashi, Y. Nabekawa, and K. Midorikawa, Generation of 10- $\mu$ J coherent extreme-ultraviolet light by use of high-order harmonics, Opt. Lett. 27, 1920 (2002).

[36] T. Popmintchev, M. C. Chen, D. Popmintchev, P. Arpin, S. Brown, S. Ališauskas, G. Andriukaitis, T. Balčiunas, O. D. Mücke, A. Pugzlys, A. Baltuška, B. Shim, S. E. Schrauth, A. Gaeta, C. Hernández-García, L. Plaja, A. Becker, A. Jaron-Becker, M. M. Murnane, and H. C. Kapteyn, Bright coherent ultrahigh harmonics in the kev x-ray regime from mid-infrared femtosecond lasers, Science 336, 1287 (2012).

[37] A. D. Shiner, C. Trallero-Herrero, N. Kajumba, H. C. Bandulet, D. Comtois, F. Legare, M. Giguere, J. C. Kieffer, P. B. Corkum, and D. M. Villeneuve, Wavelength scaling of high harmonic generation efficiency, Phys. Rev. Lett. 103, 073902 (2009). 
[38] J. Tate, T. Auguste, H. G. Muller, P. Salieres, P. Agostini, and L. F. DiMauro, Scaling of wave-packet dynamics in an intense midinfrared field, Phys. Rev. Lett. 98, 013901 (2007).

[39] E. A. Gibson, A. Paul, N. Wagner, R. Tobey, D. Gaudiosi, S. Backus, I. P. Christov, A. Aquila, E. M. Gullikson, D. T. Attwood, M. M. Murnane, and H. C. Kapteyn, Coherent soft $x$-ray generation in the water window with quasi-phase matching, Science 302, 95 (2003).

[40] M. Zepf, B. Dromey, M. Landreman, P. Foster, and S. M. Hooker, Bright quasiphase-matched soft-x-ray harmonic radiation from argon ions, Phys. Rev. Lett. 99, 143901 (2007).

[41] X. H. Zhang, A. L. Lytle, T. Popmintchev, X. B. Zhou, H. C. Kapteyn, M. M. Murnane, and O. Cohen, Quasi-phase-matching and quantum-path control of highharmonic generation using counterpropagating light, Nat. Phys. 3, 270 (2007).

[42] K. O'Keeffe, T. Robinson, and S. M. Hooker, Quasi-phase-matching high harmonic generation using trains of pulses produced using an array of birefringent plates, Opt. Express 20, 6236 (2012).

[43] A. Pirri, C. Corsi, and M. Bellini, Enhancing the yield of high-order harmonics with an array of gas jets, Phys. Rev. A 78, 011801(R) (2008).

[44] P. W. Wachulak, . Węgrzyński, Z. Zápražný, A. Bartnik, T. Fok, R. Jarocki, J. Kostecki, M. Szczurek, D. Korytár, and H. Fiedorowicz, Extreme ultraviolet tomography of multi-jet gas puff target for high-order harmonic generation, Appl. Phys. B 117, 253 (2014).

[45] A. Willner, F. Tavella, M. Yeung, T. Dzelzainis, C. Kamperidis, M. Bakarezos, D. Adams, M. Schulz, R. Riedel, M. C. Hoffmann, W. Hu, J. Rossbach, M. Drescher, N. A. Papadogiannis, M. Tatarakis, B. Dromey, and M. Zepf, Coherent control of high harmonic generation via dual-gas multijet arrays, Phys. Rev. Lett. 107, 175002 (2011).

[46] B. D. Layer, A. G. York, S. Varma, Y. H. Chen, and H. M. Milchberg, Periodic indexmodulated plasma waveguide, Opt. Express 17, 4263 (2009).

[47] C. Vozzi, M. Nisoli, J. P. Caumes, G. Sansone, S. Stagira, S. De Silvestri, M. Vecchiocattivi, D. Bassi, M. Pascolini, L. Poletto, P. Villoresi, and G. Tondello, Cluster effects in high-order harmonics generated by ultrashort light pulses, Appl. Phys. Lett. 86, 1 (2005).

[48] H. Park, Z. Wang, H. Xiong, S. B. Schoun, J. Xu, P. Agostini, and L. F. DiMauro, Sizedependent high-order harmonic generation in rare-gas clusters, Phys. Rev. Lett. 113, 263401 (2014).

[49] A. G. York, H. M. Milchberg, J. P. Palastro, and T. M. Antonsen, Direct acceleration of electrons in a corrugated plasma waveguide, Phys. Rev. Lett. 100, 195001 (2008). 
[50] S. J. Yoon, A. J. Goers, G. A. Hine, J. D. Magill, J. A. Elle, Y. H. Chen, and H. M. Milchberg, Shock formation in supersonic cluster jets and its effect on axially modulated laser-produced plasma waveguides, Opt. Express 21, 15878 (2013).

[51] K. Y. Kim, V. Kumarappan, and H. M. Milchberg, Measurement of the average size and density of clusters in a gas jet, Appl. Phys. Lett. 83, 3210 (2003).

[52] O. F. Hagena, Nucleation and growth of clusters in expanding nozzle flows, Surf. Sci. 106, 101 (1981).

[53] G. Chen, B. Kim, B. Ahn, and D. E. Kim, Pressure dependence of argon cluster size for different nozzle geometries, J. Appl. Phys. 106, 053507 (2009).

[54] R. A. Smith, T. Ditmire, and J. W. G. Tisch, Characterization of a cryogenically cooled high-pressure gas jet for laser/cluster interaction experiments, Rev. Sci. Instrum 69, 3798 (1998).

[55] K. C. Gupta, N. Jha, P. Deb, D. R. Mishra, and J. K. Fuloria, Determining the mean size and density of clusters, formed in super sonic jets, by rayleigh scattering and mach-zehnder interferometer, J. Appl. Phys. 118, 114308 (2015).

[56] T. D. Donnelly, T. Ditmire, K. Neuman, M. D. Perry, and R. W. Falcone, High-order harmonic generation in atom clusters, Phys. Rev. Lett. 76, 2472 (1996).

[57] H. Ruf, C. Handschin, R. Cireasa, N. Thire, A. Ferre, S. Petit, D. Descamps, E. Mevel, E. Constant, V. Blanchet, B. Fabre, and Y. Mairesse, Inhomogeneous high harmonic generation in krypton clusters, Phys. Rev. Lett. 110, 083902 (2013).

[58] W. Pauli, Pauli Lectures on Physics, Volume 3: Thermodynamics and the Kinetic Theory of Gases (Dover Publications, 2010).

[59] J. Fricke, Kondensation in düsenstrahlen, Physik in unserer Zeit 4, 21 (1973).

[60] S. Hoffmann, Structure and electronic properties of supported noble metal clusters, Ph.D. thesis, Technical University of Dortmund, Dortmund, Germany (2012).

[61] M. Knudsen, Die gesetze der molekularströmung und der inneren reibungsströmung der gase durch röhren, Ann. Phys. 333, 75 (1909).

[62] A. Bondi, van der waals volumes and radii, J. Phys. Chem. 68, 441 (1964).

[63] J. Anderson, Modern compressible flow : with historical perspective (McGraw-Hill, 2004).

[64] T. A. W. Wolterink, High-gradient gas-jet targets for laser wakefield acceleration, Master's thesis, University of Twente, Enschede, The Netherlands (2011).

[65] J. Couperus, A. Köhler, T. Wolterink, A. Jochmann, O. Zarini, H. Bastiaens, K. Boller, A. Irman, and U. Schramm, Tomographic characterisation of gas-jet targets for laser wakefield acceleration, Nucl. Instr. Meth. Phys. Res. Sect. A-Accel. Spectrom. Dect. Assoc. Equip. 830, 504 (2016). 
[66] V. I. Kalikmanov, J. Wolk, and T. Kraska, Argon nucleation: bringing together theory, simulations, and experiment, J. Chem. Phys. 128, 124506 (2008).

[67] R. Becker and W. Döring, Kinetische behandlung der keimbildung in übersättigten dämpfen, Ann. Phys. 416, 719 (1935).

[68] V. I. Kalikmanov, Mean-field kinetic nucleation theory, J. Chem. Phys. 124, 124505 (2006).

[69] D. Reguera and H. Reiss, Fusion of the extended modified liquid drop model for nucleation and dynamical nucleation theory, Phys. Rev. Lett. 93, 165701 (2004).

[70] J. K. Johnson, J. A. Zollweg, and K. E. Gubbins, The lennard-jones equation of state revisited, Mol. Phys. 78, 591 (1993).

[71] T. Kraska, Molecular-dynamics simulation of argon nucleation from supersaturated vapor in the nve ensemble, J. Chem. Phys. 124 (2006), 10.1063/1.2162882.

[72] R. Evans, The nature of the liquid-vapour interface and other topics in the statistical mechanics of non-uniform, classical fluids, Adv. Phys. 28, 143 (1979).

[73] O. F. Hagena, Cluster ion sources (invited), Rev. Sci. Instrum. 63, 2374 (1992).

[74] F. Dorchies, F. Blasco, T. Caillaud, J. Stevefelt, C. Stenz, A. S. Boldarev, and V. A. Gasilov, Spatial distribution of cluster size and density in supersonic jets as targets for intense laser pulses, Phys. Rev. A 68, 232011 (2003).

[75] C. Winterfeldt, C. Spielmann, and G. Gerber, Colloquium: Optimal control of highharmonic generation, Rev. Mod. Phys. 80, 117 (2008).

[76] L. A. Lompre, A. L. Huillier, M. Ferray, P. Monot, G. Mainfray, and C. Manus, Highorder harmonic-generation in xenon - intensity and propagation effects, J. Opt. Soc. Am. B-Opt. Phys. 7, 754 (1990).

[77] L. V. Keldysh, Ionization in the field of a strong electromagnetic wave, Sov. Phys. JETP 20, 1307 (1965).

[78] M. V. Ammosov, N. B. Delone, and V. P. Krainov, Tunnel ionization of complex atoms and of atomic ions in an alternating electromagnetic field, Sov. Phys. - JETP 64, 1191 (1986).

[79] M. Lewenstein, P. Salieres, and A. L'Huillier, Phase of the atomic polarization in high-order harmonic generation, Phys. Rev. A 52, 4747 (1995).

[80] J. L. Krause, K. J. Schafer, and K. C. Kulander, High-order harmonic generation from atoms and ions in the high intensity regime, Phys. Rev. Lett. 68, 3535 (1992).

[81] A. L’Huillier, M. Lewenstein, P. Salieres, P. Balcou, M. Y. Ivanov, J. Larsson, and C. G. Wahlstrom, High-order harmonic-generation cutoff, Phys. Rev. A 48, R3433 (1993). 
[82] K. S. Budil, P. Salieres, M. D. Perry, and A. L'Huillier, Influence of ellipticity on harmonic generation, Phys. Rev. A 48, R3437 (1993).

[83] R. W. Boyd, Nonlinear Optics, 2nd ed. (Academic Press, 2003).

[84] S. J. Goh, H. M. J. Bastiaens, B. Vratzov, Q. Huang, F. Bijkerk, and K. J. Boller, Fabrication and characterization of free-standing, high-line-density transmission gratings for the vacuum uv to soft x-ray range, Opt. Express 23, 4421 (2015).

[85] T. Auguste, B. Carre, and P. Salieres, Quasi-phase-matching of high-order harmonics using a modulated atomic density, Phys. Rev. A 76, R011802 (2007).

[86] P. Balcou, A. S. Dederichs, M. B. Gaarde, and A. L'Huillier, Quantum-path analysis and phase matching of high-order harmonic generation and high-order frequency mixing processes in strong laser fields, J. Phys. B 32, 2973 (1999).

[87] C. M. Heyl, J. Gudde, A. L'Huillier, and U. Hofer, High-order harmonic generation with $\mu$ J laser pulses at high repetition rates, J. Phys. B 45, 074020 (2012).

[88] T. Popmintchev, M. C. Chen, A. Bahabad, M. Gerrity, P. Sidorenko, O. Cohen, I. P. Christov, M. M. Murnane, and H. C. Kapteyn, Phase matching of high harmonic generation in the soft and hard $x$-ray regions of the spectrum, Proc Natl Acad Sci U S A 106, 10516 (2009).

[89] M. M. Fejer, D. H. Jundt, R. L. Byer, and G. A. Magel, Quasi-phase-matched second harmonic generation: Tuning and tolerances, IEEE J. Quant. Electron. 28, 2631 (1992).

[90] A. Paul, R. A. Bartels, R. Tobey, H. Green, S. Weiman, I. P. Christov, M. M. Murnane, H. C. Kapteyn, and S. Backus, Quasi-phase-matched generation of coherent extreme-ultraviolet light, Nature 421, 51 (2003).

[91] E. Constant, D. Garzella, P. Breger, E. Mevel, C. Dorrer, C. Le Blanc, F. Salin, and P. Agostini, Optimizing high harmonic generation in absorbing gases: Model and experiment, Phys. Rev. Lett. 82, 1668 (1999).

[92] B. L. Henke, E. M. Gullikson, and J. C. Davis, X-ray interactions: Photoabsorption, scattering, transmission, and reflection at $e=50-30,000 e v, z=1-92$, Atomic Data and Nuclear Data Tables 54, 181 (1993).

[93] A. Hage, B. Landgraf, M. Taylor, M. Wunsche, T. Gangolf, H. Hoppner, M. J. Prandolini, R. Riedel, M. Schulz, F. Tavella, A. Willner, M. Yeung, G. G. Paulus, C. Spielmann, B. Dromey, and M. Zepf, New design of a multi-jet target for quasi phase matching, Rev. Sci. Instrum. 85, 103105 (2014).

[94] A. Willner, F. Tavella, M. Yeung, T. Dzelzainis, C. Kamperidis, M. Bakarezos, D. Adams, R. Riedel, M. Schulz, M. C. Hoffmann, W. Hu, J. Rossbach, M. Drescher, V. S. Yakovlev, N. A. Papadogiannis, M. Tatarakis, B. Dromey, and M. Zepf, Efficient control of quantum paths via dual-gas high harmonic generation, New J. Phys. 13, 113001 (2011). 
[95] L. Z. Liu, K. O’Keeffe, and S. M. Hooker, Quasi-phase-matching of high-orderharmonic generation using polarization beating in optical waveguides, Phys. Rev. A 85, 053823 (2012).

[96] L. Z. Liu, K. O’Keeffe, and S. M. Hooker, Quasi-phase-matching of high-orderharmonic generation using multimode polarization beating, Phys. Rev. A 87, 023810 (2013).

[97] P. Tournois, Acousto-optic programmable dispersive filter for adaptive compensation of group delay time dispersion in laser systems, Opt. Commun. 140, 245 (1997).

[98] S. J. Goh, Y. Tao, P. J. M. van der Slot, H. M. J. Bastiaens, J. Herek, S. G. Biedron, M. B. Danailov, S. V. Milton, and K. J. Boller, Single-shot fluctuations in waveguided high-harmonic generation, Opt. Express 23, 24888 (2015).

[99] E. Palik, Handbook of Optical Constants of Solids (Academic Press, 1998).

[100] D. L. Windt, Imd-software for modeling the optical properties of multilayer films, Comput. Phys. 12, 360 (1998).

[101] E. Hecht, Optics (Addison-Wesley, 2002).

[102] C. G. Durfee Iii, A. R. Rundquist, S. Backus, C. Herne, M. M. Murnane, and H. C. Kapteyn, Phase matching of high-order harmonics in hollow waveguides, Phys. Rev. Lett. 83, 2187 (1999).

[103] V. P. Krainov and M. B. Smirnov, Cluster beams in the super-intense femtosecond laser pulse, Phys. Rep. 370, 237 (2002).

[104] T. Nagashima, H. Hirayama, K. Shibuya, M. Hangyo, M. Hashida, S. Tokita, and S. Sakabe, Terahertz pulse radiation from argon clusters irradiated with intense femtosecond laser pulses, Opt. Express 17, 8907 (2009).

[105] T. Ditmire, J. Zweiback, V. P. Yanovsky, T. E. Cowan, G. Hays, and K. B. Wharton, Nuclearfusion from explosions of femtosecond laser-heated deuterium clusters, Nature 398, 489 (1999).

[106] B. D. Layer, A. York, T. M. Antonsen, S. Varma, Y. H. Chen, Y. Leng, and H. M. Milchberg, Ultrahigh-intensity optical slow-wave structure, Phys. Rev. Lett. 99, 035001 (2007).

[107] K. A. Streletzky, Y. Zvinevich, B. E. Wyslouzil, and R. Strey, Controlling nucleation and growth of nanodroplets in supersonic nozzles, J. Chem. Phys. 116, 4058 (2002).

[108] S. Sinha, H. Laksmono, and B. E. Wyslouzil, A cryogenic supersonic nozzle apparatus to study homogeneous nucleation of ar and other simple molecules, Rev. Sci. Instrum. 79, 114101 (2008).

[109] H. Lixi and J. B. Young, An analytical solution for the wilson point in homogeneously nucleating flows, Proceedings - Royal Society of London, A 452, 1459 (1996). 
[110] S. Jinno, Y. Fukuda, H. Sakaki, A. Yogo, M. Kanasaki, K. Kondo, A. Y. Faenov, I. Y. Skobelev, T. A. Pikuz, A. S. Boldarev, and V. A. Gasilov, Characterization of submicronsized co2 clusters formed with a supersonic expansion of a mixed-gas using a threestaged nozzle, Appl. Phys. Lett. 102, 164103 (2013).

[111] D. G. Jang, Y. S. You, H. M. Milchberg, H. Suk, and K. Y. Kim, All-optical characterization of cryogenically cooled argon clusters in continuous gas jets, Appl. Phys. Lett. 105, 021906 (2014).

[112] X. Gao, X. Wang, B. Shim, A. V. Arefiev, R. Korzekwa, and M. C. Downer, Characterization of cluster/monomer ratio in pulsed supersonic gas jets, Appl. Phys. Lett. 100, 064101 (2012).

[113] X. Gao, A. V. Arefiev, R. C. Korzekwa, X. Wang, B. Shim, and M. C. Downer, Spatio-temporal profiling of cluster mass fraction in a pulsed supersonic gas jet by frequency-domain holography, J. Appl. Phys. 114, 034903 (2013).

[114] A. S. Boldarev, V. A. Gasilov, A. Y. Faenov, Y. Fukuda, and K. Yamakawa, Gas-cluster targets for femtosecond laser interaction: Modeling and optimization, Rev. Sci. Instrum. 77, 083112 (2006).

[115] F. B. Sprow and Prausnit.Jm, Surface tensions of simple liquids, Trans. Faraday Society 62, 1097 (1966).

[116] C. Tegeler, R. Span, and W. Wagner, A new equation of state for argon covering the fluid region for temperatures from the melting line to $700 \mathrm{k}$ at pressures up to 1000 mpa, J. Phys. Chem. Ref. Data 28, 779 (1999).

[117] M. Hipp, J. Woisetschlager, P. Reiterer, and T. Neger, Digital evaluation of interferograms, Measurement 36, 53 (2004).

[118] P. G. Hill, Condensation of water vapour during supersonic expansion in nozzles, J. Fluid Mech. 25, 593 (1966).

[119] R. Hagmeijer, Equivalence of two different integral representations of droplet distribution moments in condensing flow, Phys. Fluids 16, 176 (2004).

[120] R. Hagmeijer, R. H. A. IJzermans, and F. Put, Solution of the general dynamic equation along approximate fluid trajectories generated by the method of moments, Phys. Fluids 17, 1 (2005).

[121] D. Kashchiev, Nucleation : basic theory with applications (Butterworth Heinemann, Oxford Boston, 2000).

[122] V. I. Kalikmanov, Nucleation theory (Springer, Dordrecht New York, 2013).

[123] R. S. R. Sidin, R. Hagmeijer, and U. Sachs, Evaluation of master equations for the droplet size distribution in condensing flow, Phys. Fluids 21, 073303 (2009).

[124] P. Salières, A. L'Huillier, and M. Lewenstein, Coherence control of high-order harmonics, Phys. Rev. Lett. 74, 3776 (1995). 
[125] M. Uiberacker, T. Uphues, M. Schultze, A. J. Verhoef, V. Yakovlev, M. F. Kling, J. Rauschenberger, N. M. Kabachnik, H. Schröder, M. Lezius, K. L. Kompa, H. G. Muller, M. J. J. Vrakking, S. Hendel, U. Kleineberg, U. Heinzmann, M. Drescher, and F. Krausz, Attosecond real-time observation of electron tunnelling in atoms, Nature 446, 627 (2007).

[126] S. Witte, V. T. Tenner, D. W. E. Noom, and K. S. E. Eikema, Lensless diffractive imaging with ultra-broadband table-top sources: from infrared to extreme-ultraviolet wavelengths, Light-Sci Appl 3, e163 (2014).

[127] X. He, M. Miranda, J. Schwenke, O. Guilbaud, T. Ruchon, C. Heyl, E. Georgadiou, R. Rakowski, A. Persson, M. B. Gaarde, and A. L'Huillier, Spatial and spectral properties of the high-order harmonic emission in argon for seeding applications, Phys. Rev. A 79 (2009).

[128] S. Hadrich, A. Klenke, J. Rothhardt, M. Krebs, A. Hoffmann, O. Pronin, V. Pervak, J. Limpert, and A. Tunermann, High photon flux table-top coherent extremeultraviolet source, Nat. Photon. 8, 779 (2014).

[129] S. Ghimire, A. D. Dichiara, E. Sistrunk, P. Agostini, L. F. Dimauro, and D. A. Reis, Observation of high-order harmonic generation in a bulk crystal, Nat. Phys. 7, 138 (2011).

[130] G. Vampa, T. J. Hammond, N. Thire, B. E. Schmidt, F. Legare, C. R. McDonald, T. Brabec, D. D. Klug, and P. B. Corkum, All-optical reconstruction of crystal band structure, Phys. Rev. Lett. 115, 193603 (2015).

[131] G. Ndabashimiye, S. Ghimire, M. Wu, D. A. Browne, K. J. Schafer, M. B. Gaarde, and D. A. Reis, Solid-state harmonics beyond the atomic limit, Nature 534, 520 (2016).

[132] M. X. Wu, S. Ghimire, D. A. Reis, K. J. Schafer, and M. B. Gaarde, High-harmonic generation from bloch electrons in solids, Phys. Rev. A 91, 043839 (2015).

[133] J. W. G. Tisch, T. Ditmire, D. J. Fraser, N. Hay, M. B. Mason, E. Springate, J. P. Marangos, and M. H. R. Hutchinson, Investigation of high-harmonic generation from xenon atom clusters, J. Phys. B 30, L709 (1997).

[134] M. Aladi and I. B. Foldes, High harmonic generation from atom clusters, JPCS 508, 012016 (2014).

[135] M. Aladi, R. Bolla, P. Racz, and I. B. Foldes, Noble gas clusters and nanoplasmas in high harmonic generation, Nucl. Instrum. Meth. B 369, 68 (2016).

[136] U. Saalmann, Resonant energy absorption of rare-gas clusters in strong laser pulses, J. Mod. Opt. 53, 173 (2006).

[137] F. Quere, C. Thaury, P. Monot, S. Dobosz, P. Martin, J. P. Geindre, and P. Audebert, Coherent wake emission of high-order harmonics from overdense plasmas, Phys. Rev. Lett. 96, 125004 (2006). 
[138] Y. Tao, R. Hagmeijer, E. T. A. van der Weide, H. M. J. Bastiaens, and K. J. Boller, Revisiting argon cluster formation in a planar gas jet for high-intensity laser matter interaction, J. Appl. Phys. 119, 164901 (2016).

[139] B. Shim, G. Hays, R. Zgadzaj, T. Ditmire, and M. C. Downer, Enhanced harmonic generation from expanding clusters, Phys. Rev. Lett. 98, 123902 (2007).

[140] D. F. Zaretsky, P. Korneev, and W. Becker, High-order harmonic generation in clusters irradiated by an infrared laser field of moderate intensity, J. Phys. B 43, 105402 (2010).

[141] U. Saalmann, S. Ch, and J. M. Rost, Mechanisms of cluster ionization in strong laser pulses, Journal of Physics B: Atomic, Molecular and Optical Physics 39, R39 (2006).

[142] K. Hoffmann, B. Murphy, N. Kandadai, B. Erk, A. Helal, J. Keto, and T. Ditmire, Rare-gas-cluster explosions under irradiation by intense short xuv pulses, Phys. Rev. A 83, 043203 (2011).

[143] Y. Mairesse, J. Higuet, N. Dudovich, D. Shafir, B. Fabre, E. Mével, E. Constant, S. Patchkovskii, Z. Walters, M. Y. Ivanov, and O. Smirnova, High harmonic spectroscopy of multichannel dynamics in strong-field ionization, Phys. Rev. Lett. 104, 213601 (2010).

[144] S. Baker, J. S. Robinson, C. A. Haworth, H. Teng, R. A. Smith, C. C. Chirilă, M. Lein, J. W. G. Tisch, and J. P. Marangos, Probing proton dynamics in molecules on an attosecond time scale, Science 312, 424 (2006).

[145] S. Kazamias, D. Douillet, C. Valentin, F. Weihe, F. Auge, T. Lefrou, G. Grillon, S. Sebban, and P. Balcou, Observation of high-contrast coherence fringes in high-order harmonic generation, Phys. Rev. A 68, 033819 (2003).

[146] W. F. Chan, G. Cooper, X. Guo, G. R. Burton, and C. E. Brion, Absolute optical oscillator strengths for the electronic excitation of atoms at high resolution. iii. the photoabsorption of argon, krypton, and xenon, Phys. Rev. A 46, 149 (1992).

[147] S. Kazamias, S. Daboussi, O. Guilbaud, K. Cassou, D. Ros, B. Cros, and G. Maynard, Pressure-induced phase matching in high-order harmonic generation, Phys. Rev. A 83, 063405 (2011).

[148] S. Kazamias, D. Douillet, F. Weihe, C. Valentin, A. Rousse, S. Sebban, G. Grillon, F. Augé, D. Hulin, and P. Balcou, Global optimization of high harmonic generation, Phys. Rev. Lett. 90, 193901 (2003).

[149] K. Midorikawa, Y. Nabekawa, and A. Suda, Xuv multiphoton processes with intense high-order harmonics, Prog. Quant. Electron. 32, 43 (2008).

[150] P. Rudawski, C. M. Heyl, F. Brizuela, J. Schwenke, A. Persson, E. Mansten, R. Rakowski, L. Rading, F. Campi, B. Kim, P. Johnsson, and A. L'Huillier, A highflux high-order harmonic source, Rev. Sci. Instrum. 84, 073103 (2013). 
[151] Y. Tamaki, J. Itatani, M. Obara, and K. Midorikawa, Optimization of conversion efficiency and spatial quality of high-order harmonic generation, Phys. Rev. A 62, 063802 (2000).

[152] P. M. Paul, E. S. Toma, P. Breger, G. Mullot, F. Augé, P. Balcou, H. G. Muller, and P. Agostini, Observation of a train of attosecond pulses from high harmonic generation, Science 292, 1689 (2001).

[153] L. Z. Liu, K. O’Keeffe, and S. M. Hooker, Optical rotation quasi-phase-matching for circularly polarized high harmonic generation, Opt. Lett. 37, 2415 (2012).

[154] H. Singhal, R. A. Ganeev, P. A. Naik, J. A. Chakera, U. Chakravarty, H. S. Vora, A. K. Srivastava, C. Mukherjee, C. P. Navathe, S. K. Deb, and P. D. Gupta, High-order harmonic generation in a plasma plume of in situ laser-produced silver nanoparticles, Phys. Rev. A 82, 043821 (2010).

[155] Y. Y. Yang, A. Scrinzi, A. Husakou, Q. G. Li, S. L. Stebbings, F. Süßmann, H. J. Yu, S. Kim, E. Rühl, J. Herrmann, X. C. Lin, and M. F. Kling, High-harmonic and single attosecond pulse generation using plasmonic field enhancement in ordered arrays of gold nanoparticles with chirped laser pulses, Opt. Express 21, 2195 (2013).

[156] S. L. Stebbings, F. Suessmann, Y. Y. Yang, A. Scrinzi, M. Durach, A. Rusina, M. I. Stockman, and M. F. Kling, Generation of isolated attosecond extreme ultraviolet pulses employing nanoplasmonic field enhancement: optimization of coupled ellipsoids, New J. Phys. 13, 073010 (2011).

[157] A. Husakou and J. Herrmann, Quasi-phase-matched high-harmonic generation in composites of metal nanoparticles and a noble gas, Phys. Rev. A 90, 023831 (2014).

[158] R. Reid, The properties of gases and liquids (McGraw-Hill, New York, 1987).

[159] R. B. Stewart and R. T. Jacobsen, Thermodynamic properties of argon from the triple point to 1200-k with pressures to 1000-mpa, J. Phys. Chem. Ref. Data 18, 639 (1989). 


\section{LIST OF PUBLICATIONS}

\section{JOURNALS}

- Y. Tao, R. Hagmeijer, E. T. A. van der Weide, H. M. J. Bastiaens and K. -J. Boller, Revisiting argon cluster formation in a planar gas jet for high-intensity laser matter interaction, J. Appl. Phys 119, 164901 (2016).

- Y. Tao, S. J. Goh, H. M. J. Bastiaens, P. J. M. van der Slot, S. G. Biedron, S. V. Milton and K. -J. Boller, Cluster size dependence of high-order harmonic generation, submitted.

- Y. Tao, S. J. Goh, H. M. J. Bastiaens, P. J. M. van der Slot, S. G. Biedron, S. V. Milton and K. -J. Boller, A temporal quasi-phase matching model for high-order harmonic generation, submitted.

- S. J. Goh, J. Reinink, Y. Tao, P. J. M. van der Slot, H. M. J. Bastiaens, J. L. Herek, S. G. Biedron, S. V. Milton and K. -J. Boller, Spectral control of high-harmonic generation via drive laser pulse shaping in a wide-diameter capillary, Opt. Express 24, 1604 (2016).

- S. J. Goh, Y. Tao, P. J. M. van der Slot, H. M. J. Bastiaens, J. L. Herek, S. G. Biedron, M. B. Danailov, S. V. Milton and K. -J. Boller, Single-shot fluctuations in waveguided high-harmonic generation, Opt. Express 23, 24888 (2015).

\section{CONFERENCE PROCEEDINGS}

- S. J. Goh, Y. Tao, P. J. M. van der Slot, H. M. J. Bastiaens, S. G. Biedron, S. V. Milton and K. -J. Boller, High-harmonic source for seeding of FERMI@Elettra, Proceedings of FEL 2010, Malmö, Sweden TUPB04, p.262-265 (2010).

\section{ORAL PRESENTATIONS}

- Y. Tao, S. J. Goh, P. J. M. van der Slot, H. M. J. Bastiaens, F. A. van Goor, E. T. A. van der Weide, R. Hagmeijer, J. L. Herek, S. G. Biedron, S. V. Milton, M. B. Danailov and K. -J. Boller, Characterization of an axis-density modulated plasma waveguide based on laser-cluster interaction, Deutsche Physikalische Gesellschaft Frühjahrstagung (DPG-Frühjahrstagung Sektion AMOP), Hannover, Germany (18-22 March 2013). 


\section{POSTER PRESENTATIONS}

- Y. Tao, S. J. Goh, P. J. M. van der Slot, H. M. J. Bastiaens and K. -J. Boller, A temporal model for quasi-phase matching in high-order harmonic generation, $40^{\text {th }}$ Annual meeting NNV AMO Lunteren), Lunteren, The Netherlands (10-11 October 2016).

- Y. Tao, S. J. Goh, P. J. M. van der Slot, H. M. J. Bastiaens and K. -J. Boller, Investigation of high harmonic generation in a density modulated argon cluster jet, Physics@FOM Veldhoven conference), Veldhoven, The Netherlands (19-20 January 2016).

- Y. Tao, S. J. Goh, P. J. M. van der Slot, H. M. J. Bastiaens and K. -J. Boller, Quasi-phase matching high harmonic generation in a density modulated argon cluster jet, $39^{\text {th }}$ Annual meeting NNV AMO Lunteren), Lunteren, The Netherlands (06-07 October 2015).

- Y. Tao, S. J. Goh, P. J. M. van der Slot, H. M. J. Bastiaens, F. A. van Goor, E. T. A. van der Weide, R. Hagmeijer, S. G. Biedron, S. V. Milton, M. B. Danailov, J. L. Herek and K. -J. Boller, Cluster size and density re-distribution by a rod obstacle preparing for quasi-phase matching of high harmonic generation, $38^{\text {th }}$ Annual meeting NNV AMO Lunteren), Lunteren, The Netherlands (07-08 October 2014).

- Y. Tao, S. J. Goh, P. J. M. van der Slot, H. M. J. Bastiaens, F. A. van Goor, E. T. A. van der Weide, R. Hagmeijer, S. G. Biedron, S. V. Milton, M. B. Danailov, J. L. Herek and K. -J. Boller, Towards a density-modulated argon-plasma waveguide for quasi phase-matching of high-harmonic generation, $37^{\text {th }}$ Annual meeting NNV AMO Lunteren), Lunteren, The Netherlands (01-02 October 2013).

- Y. Tao, S. J. Goh, P. J. M. van der Slot, H. M. J. Bastiaens, F. A. van Goor, E. T. A. van der Weide, R. Hagmeijer, S. G. Biedron, S. V. Milton, M. B. Danailov, J. L. Herek and K. -J. Boller, Cluster Jets with a Periodic Density Modulation for Quasi-Phase Matching of High-Harmonic Generation, MESA+ Annual meeting), Enschede, The Netherlands (16 September 2013).

- Y. Tao, S. J. Goh, P. J. M. van der Slot, H. M. J. Bastiaens, F. A. van Goor, E. T. A. van der Weide, R. Hagmeijer, S. G. Biedron, S. V. Milton, M. B. Danailov, J. L. Herek and K. -J. Boller, Spatially resolved cluster size and density measurement in an axis modulated argon jet, $25^{\text {th }}$ Annual meeting NNV CPS Lunteren), Lunteren, The Netherlands (05-06 March 2013).

- Y. Tao, S. J. Goh, P. J. M. van der Slot, H. M. J. Bastiaens, F. A. van Goor, E. T. A. van der Weide, R. Hagmeijer, S. G. Biedron, S. V. Milton, M. B. Danailov, J. L. Herek and K. -J. Boller, Spatially resolved cluster size and density measurement in an argon jet, Physics@FOM Veldhoven conference), Veldhoven, The Netherlands (21-22 January 2013).

- Y. Tao, S. J. Goh, P. J. M. van der Slot, H. M. J. Bastiaens, S. Franzan, R. Hagmeijer, S. G. Biedron, S. V. Milton, M. B. Danailov, J. L. Herek and K. -J. Boller, Spatially resolved cluster size and density measurement in an argon jet, $36^{\text {th }}$ Annual meeting NNV AMO Lunteren), Lunteren, The Netherlands (08-09 October 2012). 
- Y. Tao, S. J. Goh, P. J. M. van der Slot, H. M. J. Bastiaens, F. A. van Goor, E. T. A. van der Weide, R. Hagmeijer, S. G. Biedron, S. V. Milton, M. B. Danailov, J. L. Herek and K. -J. Boller, Extreme ultraviolet source based on high harmonic generation from ions for the direct seeding of Free Electron Lasers, $24^{\text {th }}$ Annual meeting NNV CPS Lunteren), Lunteren, The Netherlands (13-14 March 2012).

- Y. Tao, S. J. Goh, P. J. M. van der Slot, H. M. J. Bastiaens, F. A. van Goor, E. T. A. van der Weide, R. Hagmeijer, S. G. Biedron, S. V. Milton, M. B. Danailov, J. L. Herek and K. -J. Boller, An ion based high harmonic source for the direct seeding of free electron lasers in the soft $x$-ray regime, The $49^{\text {th }}$ course Atoms and Plasmas in Super Intense Laser fields, Erice, Italy (14-24 July 2011). 



\section{ACKNOWLEDGEMENTS}

My Ph.D life in University of Twente is unique and amazing. It lasted more than six years. During the last six years, a large number of people have contributed to my work as well as my life in Enschede, and helped me in many different ways. Please forgive me if I forget to mention some people.

First of all, I would like to thank my supervisor and promoter Klaus Boller for giving me the opportunity to do this research in the Laser Physics and Nonlinear Optics (LPNO) group. During our discussions, I was always inspired by his brilliant suggestions and his deep understanding of the physics behind the problems. The way he explain complicated phenomenon in physics with just a pen and a piece of paper always amazed me. Furthermore, I also learned a lot from him how to carefully and accurately choose English words during my writing. Moreover, I am very grateful for the extra half-year financial support from the group.

My co-promoter and daily supervisor Bert Bastiaens deserves my special gratitude. I really appreciated that we worked together not only in the office but also in the lab. His critical attitude towards my research and tendency to deliberately misinterpret any unclear sentences which I wrote are very helpful. It raises the quality of my paper and thesis, and also significantly improves my scientific writing skills. I will always remember the day when we finished Chapter 5 at 4 o'clock in the morning.

I would like to acknowledge all the other committee members: Prof. dr. M. Kovacev, Prof. dr. C. Fallnich, Prof. dr. H.P. Urbach, Prof. dr. J.L. Herek and Prof. dr. P.W.H. Pinkse. Thank you for agreeing to be a member in the committee and taking the time and effort to evaluate this thesis.

Dr. ir. R. Hagmeijer from Engineering Fluid Dynamics group deserves my special thanks for his huge contribution to this thesis as well as our first paper. His model on supersonic gas expansion and cluster formation is extremely helpful. Thank you for sharing all these knowledge with us. Furthermore, as an experimentalist, I also learned how to tackle scientific problems as a theoretician like him.

I would like to specifically thank our former technician Gerard. Without his help, the experiments presented in this thesis can not be accomplished. His practical skills on mechanics such as building up our huge vacuum system are amazing. Most importantly, I really appreciate that he always came to help me promptly after I phoned him about the "accidents" which I had in the lab.

Many thanks go to my paranymphs Jesse and Ron. Jesse was always patient and willing to help me, even when he was busy with his own work. I also enjoyed working together with him with our Ti:Sa laser system in the lab. Ron and I do enjoy working on practical issues in the lab, such as fixing the broken cables and machining mechanical parts for the vacuum system. He was always willing to point out my grammar and spelling mistakes in English, which I appreciated a lot. 
I would also express my gratitude to my colleague Tom for his critical review on each chapter of my thesis and his patience in proving the entire manuscript.

Many thanks go to other members in my research group for lots of interesting discussions and an awesome working atmosphere during the past few years. I enjoyed every group meeting, coffee break, Friday-afternoon-drink, Ph.D-dinner (btw, we should restart it soon), and group outing. So thank you: Ab, Ale, Carin, Caterina, Caterina (the "Queen"), Chris, Dave, Edith, Filip, Fred, Hannah, Ingrid, Jean, Jelle, Joanna, Joliene, Jonathan, Jörn, Kasper, Kai, Lennart, Marc, Marco, Mathijs, Muharrem, Paul, Peter, Reinier, Rob, Robert, Ruud, Sean, Simone, Sjored, Thomas, Victoria, Wiebke, Youwen, Yvan. Especially, thank you very much for listening to my weekly "funny" jokes.

I also want to extend my gratitude to the members of the Complex Photonic Systems group (Willem, Allard, Ravitej, Diana, Tristan, Evangelos, Bas, Jin, Sergei, Henry, etc.) the Optical Sciences group (Herman, Jeroen, Frans, Eric, Andrew, Jinfeng, Jorick, Gerwin, Yean-Sheng, etc.) and other people from ANP (Fred, Lantian, Feng, etc), for the use of their equipment and valuable scientific discussions.

Over the last six years, I lived at two awesome flats, Carpe Noctem and Room 77 (okay, only I call it room 77). In Carpe Noctem, I was extremely lucky to join such a Dutch style student flat as a foreigner. I experienced three-year typical Dutch-student life. I fondly remember the Chrismas dinners, Sinterklaas celebrations, flat weekends and other activities. All in all, I had a great time with all of them: Liza, Steven, Franka, Arjen, Cindy, Richte, Randy, Monica, Liesbeth, Sjored, Michael, Jarka, Hylke, Kamiel, etc. Of course, I should not forget to mention Gert-jan and Wiljan. Our three-person team is awesome and I enjoyed to hangout with them during the last six years. Road trips to Italy and Hanover are legendary and unforgettable. Ik ben geweldig!

For the last two years, I moved to Room 77 together with Niya (Wei Ya), Liqun Xie and our frequent guest Yue Ma. We had lots of fruitful discussions about the politics and lifestyle in China during the dinner (always cooked by Niya, he is too kind), which I enjoyed very much. Moreover, I am very glad that all of them joined my cycling team. We cycled in a total distance of more than two thousands kilometers with other members (Yi Wang, Aijie Liu and Song Ma) during last two years. Moreover, I really enjoyed watching this year's Giro d'italia in Nijmegen together with them. And I promise that one day we will definitely go to France for the Tour.

Besides my flatmates, I would like to thank all the guys in our Enschede Chinese Old Boy football club for the wonderful time we spent during each Sunday afternoon. So thank you: Captain Zhou Lu, Chao Wang, He Huang, Lei Wang, Xingwu Sun, Tianpei Wang, Xiang Zhang, Jianfeng Huang, Donghai Zheng, Min Jia, etc. Moreover, Pengkun Chen and Chen Ling deserve my thanks, I enjoyed playing basketball and running with you before I joined the football club.

I would like to acknowledge a ten-person weekend group which I joined in 2014. I enjoyed the every weekend we spent together with a lot of funny discussions and games. So thank you: Luman Zhan, Letian Wang, Xiaohua Li, Kenan Niu, Wenlong Chen, Rong Wang, Fangzhou He. Of course, I should not forget to mention the rest two members Ye Wei (sorry, Yu Fan), a great future scientist, and Yiyuan Lin, a great CTO as well as my drinking buddy.

Tak Sing Chan also deserves my special gratitude, since he helped me a lot when I 
just arrived in Enschede in 2010 and introduced me several nice friends who lived in Macandra and later in his new house. So thank you: Haishan Cao, Wanshu Zhang, Peng Gao, Yang Cui, Dongfang Liu, Zhao Wang, Wei Fang, Bingxi Xue, Yannan Cheng, Peng Jin, Xiaochen Zhang, Yihan Huang, Lu Xu, etc.

I would also like to acknowledge Zhen Chen. Somehow, I found we have a lot of common hobbies and interests. I really enjoyed visiting him (also later together with Qing Shen) many times in Leiden (far away, but fun), the best city in the Netherlands. Also thank you very much for providing me the place to stay over when I needed to take an early flight from Schiphol.

I also want to extend my gratitude to all my friends in Shanghai and Hong Kong. They always cheered me up through internet, when I was frustrated with my research. Especially, I would like to thank Xiaosong Yan who visited me several times in the Netherlands and Qincheng Wang who invited me to the trips in Brug, Brussels, Amsterdam, The Hague and Lisbon.

My special thanks go out to Prof. S.K. Hark (my former supervisor in CUHK), Yiu Ho Tam and Prof. M.C. Chu. Thank you very much for your support from Hong Kong. In particular, I want to express my gratitude to Prof. Chu for providing me the chance to visit CERN, one of the most complex, large facilities in the world, in Geneva with CU colleagues.

Last but not least, I would like to thank all my family members who have been unceasingly supportive and encouraging. 